\title{
Localization for Wilson Loops in
}

\section{Chern-Simons Theory}

\author{
Chris Beasley \\ Department of Mathematics, Northeastern University, Boston, MA 02115, \\ USA
}

\begin{abstract}
We reconsider Chern-Simons gauge theory on a Seifert manifold $M$, which is the total space of a non-trivial circle bundle over a Riemann surface $\Sigma$, possibly with orbifold points. As shown in previous work with Witten, the path integral technique of non-abelian localization can be used to express the partition function of Chern-Simons theory in terms of the equivariant cohomology of the moduli space of flat connections on $M$. Here we extend this result to apply to the expectation values of Wilson loop operators that wrap the circle fibers of $M$ over $\Sigma$. Under localization, such a Wilson loop operator reduces naturally to the Chern character of an associated universal bundle over the moduli space. Along the way, we demonstrate that the stationary-phase approximation to the Wilson loop path integral is exact for torus knots in $S^{3}$, an observation made empirically by Lawrence and Rozansky prior to this work.
\end{abstract}

\section{Introduction}

An outstanding issue in the study of Chern-Simons gauge theory has long been to understand more precisely the Lagrangian formulation of this theory via the Feynman path integral, in terms of which the partition function $Z$ at

e-print archive: http://lanl.arXiv.org/abs/0911.2687 
level $k$ is described formally as an integral over the space of all connections $A$ on a given three-manifold $M$,

$$
Z(k)=\int \mathcal{D} A \exp \left[\mathrm{i} \frac{k}{4 \pi} \int_{M} \operatorname{Tr}\left(A \wedge d A+\frac{2}{3} A \wedge A \wedge A\right)\right] .
$$

Of course, as explained by Witten [105] in his foundational work on ChernSimons theory, the Hamiltonian formalism can be alternatively applied to give a completely rigorous [95] and explicitly computable description of the Chern-Simons partition function in terms of two-dimensional rational conformal field theory and a "cut-and-paste" presentation of $M$ via surgery on links in $S^{3}$. Yet despite its computability, this algebraic definition of $Z(k)$ as a quantum three-manifold invariant obscures many features which are manifest in the preceding path integral and which one would like to understand more deeply.

As a simple example, in the semi-classical limit that $k$ is large, a naive stationary-phase approximation can be applied to the path integral, and this approximation implies asymptotic behavior for $Z(k)$ that is far from evident in the complicated, exact expressions that arise from conformal field theory - an observation recently emphasized by Freed in [50]. Nonetheless, the predicted asymptotic behavior can be checked in examples, as was done early on by Freed and Gompf [51], Jeffrey [62], and Garoufalidis [69]. See [1] for related analysis, and see Section 7 in [91] for a survey of continuing work in this area.

For many topological quantum field theories, including most notably topological Yang-Mills theory in dimensions two and four (reviewed nicely in [29]), the semi-classical approximation to the path integral is actually exact, due to the presence of a conserved, nilpotent scalar supercharge which is interpreted formally as a Becchi-Rouet-Stora-Tyutin (BRST) operator in the theory. As a result, the path integral in those examples reduces to the integral of an appropriate cohomology class over a finite-dimensional moduli space of supersymmetric solutions.

In the happy circumstance above, the partition function can be described not only algebraically in the Hamiltonian formalism, as for any topological quantum field theory, but also cohomologically in the Lagrangian formalism, and one might hope to benefit by comparing the two descriptions. Indeed, in a spectacular application, Witten [108] used a Hamiltonian computation, originally due to Migdal [84], of the two-dimensional Yang-Mills partition function to deduce via its path integral interpretation very general results about the cohomology ring of the moduli space of flat connections on a Riemann surface. 
From this perspective, one might wonder to what extent, if any, the topological observables in Chern-Simons theory also admit a cohomological interpretation of the sort that arises naturally in Yang-Mills theory.

The present paper is a sequel to our earlier work [18] with Witten in which we gave a partial answer to the preceding question. There we demonstrated that the partition function of Chern-Simons theory on a threemanifold $M$ does admit a cohomological interpretation in the special case that $M$ is a Seifert manifold. Such a three-manifold can be described succinctly as the total space of a non-trivial circle bundle over a Riemann surface $\Sigma$,

$$
\begin{gathered}
S^{1} \longrightarrow \\
\\
\qquad \begin{array}{l}
\downarrow \\
\Sigma
\end{array},
\end{gathered}
$$

where $\Sigma$ is generally allowed to have orbifold points and the circle bundle is allowed to be a corresponding orbifold bundle.

Specifically, the results in [18] were based upon the technique of nonabelian localization as applied to the Chern-Simons path integral in (1.1). Very briefly, non-abelian localization provides a cohomological interpretation for a special class of symplectic integrals which are intimately related to symmetries. These integrals take the canonical form

$$
Z(\epsilon)=\int_{X} \exp \left[\Omega-\frac{1}{2 \epsilon}(\mu, \mu)\right] .
$$

Here $X$ is an arbitrary symplectic manifold with symplectic form $\Omega$. We assume that a Lie group $H$ acts on $X$ in a Hamiltonian fashion with moment map $\mu: X \rightarrow \mathfrak{h}^{*}$, where $\mathfrak{h}^{*}$ is the dual of the Lie algebra $\mathfrak{h}$ of $H$. We also introduce an invariant quadratic form $(\cdot, \cdot)$ on $\mathfrak{h}$ and dually on $\mathfrak{h}^{*}$ to define the function $S=\frac{1}{2}(\mu, \mu)$ appearing in the integrand of $Z(\epsilon)$. Finally, $\epsilon$ is a coupling parameter.

Although it is far from evident that the path integral in (1.1) bears any relation to the canonical symplectic integral in (1.3), we nevertheless explained in [18] how to recast the Chern-Simons path integral as such a symplectic integral when $M$ is a Seifert manifold. Given this initial and somewhat miraculous step, general facts about non-abelian localization were then enough to imply the exactness of the semi-classical approximation to the Chern-Simons path integral on a Seifert manifold, a result obtained empirically from known formulae for $Z(k)$ by Lawrence and Rozansky [74] and Mariño [81] prior to our work. Finally, using localization we obtained a 
precise description of the Chern-Simons partition function in terms of the equivariant cohomology of the moduli space of flat connections on $M$.

At this point, an obvious further question to ask is whether non-abelian localization can be applied to give an exact, cohomological description for any other quantities in Chern-Simons theory beyond the partition function. Of course, the other quantities in question must be the expectation values of Wilson loop operators, and the purpose of this paper is to explain how to apply non-abelian localization to analyze the Chern-Simons path integral including Wilson loop insertions.

We recall that a Wilson loop operator $W_{R}(C)$ in any gauge theory on a manifold $M$ is described by the data of an oriented, closed curve $C$ which is smoothly embedded in $M$ and which is decorated by an irreducible representation $R$ of the gauge group $G$. As a classical functional of the connection $A$, the Wilson loop operator is then given simply by the trace in $R$ of the holonomy ${ }^{1}$ of $A$ around $C$,

$$
W_{R}(C)=\operatorname{Tr}_{R} P \exp \left(-\oint_{C} A\right)
$$

To describe the expectation value of $W_{R}(C)$ in the Lagrangian formulation of Chern-Simons theory, we introduce the absolutely normalized Wilson loop path integral

$$
Z(k ; C, R)=\int \mathcal{D} A W_{R}(C) \exp \left[\mathrm{i} \frac{k}{4 \pi} \int_{M} \operatorname{Tr}\left(A \wedge d A+\frac{2}{3} A \wedge A \wedge A\right)\right],
$$

in terms of which the Wilson loop expectation value is given by the ratio

$$
\left\langle W_{R}(C)\right\rangle=\frac{Z(k ; C, R)}{Z(k)} .
$$

Just as for the partition function, the Wilson loop path integral (1.5) can be computed exactly using the Hamiltonian formulation of Chern-Simons theory and its relation to rational conformal field theory. In that approach, the expectation values of Wilson loop operators lead naturally [105] to knot invariants such as the celebrated Jones polynomial.

In this paper, our perspective on the Wilson loop operator is rather different. Here we wish to apply non-abelian localization to the Wilson loop

\footnotetext{
${ }^{1}$ Since we work in conventions for which $d_{A}=d+A$ is the covariant derivative, the holonomy of $A$ around $C$ is given by $P \exp \left(-\oint_{C} A\right)$, with the minus sign as above.
} 
path integral in (1.5) to obtain a new, complementary description of $W_{R}(C)$ as a cohomology class on the moduli space of flat connections on $M$.

\subsection{Some experimental evidence}

At the outset, it is again far from clear that the Wilson loop path integral bears any relation to the canonical symplectic integral in (1.3) to which nonabelian localization applies. To present one suggestive piece of evidence, let us consider the simplest Wilson loop - namely, the unknot Wilson loop - in Chern-Simons theory on $S^{3}$ with gauge group $S U(2)$. Irreducible representations of $S U(2)$ are uniquely labeled by their dimension, and we let $j$ denote the irreducible representation of $S U(2)$ with dimension $j$.

For the unknot, the absolutely-normalized Wilson loop path integral in (1.5) is given exactly by

$$
Z(k ; \bigcirc, j)=\sqrt{\frac{2}{k+2}} \sin \left(\frac{\pi j}{k+2}\right), \quad j=1, \ldots, k+1 .
$$

As indicated, $j$ runs without loss over the finite set of irreducible representations which are integrable in the $S U(2)$ current algebra at level $k$. This simple result was first obtained by Witten in [105] using the Hamiltonian formulation of Chern-Simons theory, and as a special case, when $\mathbf{j}=\mathbf{1}$ is trivial, the general formula for $Z(k ; \bigcirc, \mathbf{j})$ reduces to the standard expression for the $S U(2)$ partition function $Z(k)$ of Chern-Simons theory on $S^{3}$.

From the semi-classical perspective, we can gain greater insight into the exact formula for $Z(k ; \bigcirc, \mathbf{j})$ by rewriting $(1.7)$ as a contour integral over the real axis,

$$
\begin{aligned}
Z(k ; \bigcirc, \mathbf{j})= & \frac{1}{2 \pi \mathrm{i}} \mathrm{e}^{-\frac{\mathrm{i} \pi\left(1+j^{2}\right)}{2(k+2)}} \int_{-\infty}^{+\infty} d x \operatorname{ch}_{\mathrm{j}}\left(\mathrm{e}^{\frac{\mathrm{i} \pi}{4}} \frac{x}{2}\right) \sinh ^{2}\left(\mathrm{e}^{\frac{\mathrm{i} \pi}{4}} \frac{x}{2}\right) \\
& \times \exp \left(-\frac{(k+2)}{8 \pi} x^{2}\right) .
\end{aligned}
$$

Here $\mathrm{ch}_{\mathbf{j}}$ is the character of $S U(2)$ associated to the representation $\mathbf{j}$,

$$
\operatorname{ch}_{\mathbf{j}}(y)=\frac{\sinh (j y)}{\sinh (y)}=\mathrm{e}^{(j-1) y}+\mathrm{e}^{(j-3) y}+\cdots+\mathrm{e}^{-(j-3) y}+\mathrm{e}^{-(j-1) y},
$$

and the equality between the expressions in (1.7) and (1.8) follows by evaluating (1.8) as a sum of elementary Gaussian integrals. 
Now, the only flat connection on $S^{3}$ is the trivial connection, and for the case of the partition function $Z(k)$, we explained previously [18] how the contour integral over $x$ in (1.8) can be interpreted very precisely as the stationary-phase contribution from the trivial connection to the ChernSimons path integral. From a geometric perspective, the contour integral arises as an integral over the Cartan subalgebra of $S U(2)$, regarded as the group of constant gauge transformations on $S^{3}$. The constant gauge transformations are the stabilizer of the trivial connection in the group of all gauge transformations, and the presence of this stabilizer group plays an important role in the semi-classical analysis of the Chern-Simons path integral. In any event, as evident from (1.8), the stationary-phase approximation to the Chern-Simons path integral on $S^{3}$ is exact.

Given this interpretation of $Z(k ; \bigcirc, \mathbf{j})$ in the special case $\mathbf{j}=\mathbf{1}$, it is very tempting to apply the same interpretation for arbitrary $\mathbf{j}$, so that the contour integral over $x$ in (1.8) more generally represents the stationary-phase contribution from the trivial connection to the Wilson loop path integral in (1.5). Moreover, since all dependence on the representation $\mathbf{j}$ enters the integrand of (1.8) through the $S U(2)$ character $\mathrm{ch}_{\mathbf{j}}$, we naturally identify $\mathrm{ch}_{\mathbf{j}}$ as the avatar of the unknot Wilson loop operator itself when the path integral in (1.5) is reduced to the contour integral in (1.8).

The semi-classical identification of the unknot Wilson loop operator with a character is quite elegant, and one of our eventual results will be to obtain this identification directly from the path integral via non-abelian localization. However, the unknot Wilson loop in $S^{3}$ is also very special. For instance, if we regard $S^{3}$ as a Seifert manifold by virtue of the Hopf fibration over $S^{2}$,

$$
\begin{array}{r}
S^{1} \longrightarrow S^{3} \\
\downarrow^{\pi}, \\
S^{2}
\end{array}
$$

then the unknot can be represented by one of the $S^{1}$ fibers in (1.10). As a result, the unknot Wilson loop is the unique Wilson loop in $S^{3}$ which respects the distinguished $U(1)$ action rotating the fibers of (1.10).

To apply non-abelian localization to the Wilson loop path integral on a general Seifert manifold $M$, we must similarly assume that the Wilson loop operator respects the $U(1)$ action on $M$, which rotates the fibers in (1.2). As for the unknot, such a Wilson loop operator necessarily wraps a Seifert fiber of $M$. To avoid potential confusion later, we refer to the Wilson loops wrapping Seifert fibers of $M$ as "Seifert loops" to distinguish them from arbitrary Wilson loops in $M$, about which we will also have some things to say. 
Perhaps surprisingly, even for $S^{3}$ the unknot is not the only knot which a Seifert loop operator can wrap. As we recall in Section 7.1, $S^{3}$ admits infinitely many distinct Seifert presentations as the total space of a non-trivial circle bundle over a Riemann surface of genus zero with two orbifold points, of relatively-prime orders $\mathbf{p}$ and $\mathbf{q}$. The associated Seifert loop operator then wraps a $(\mathbf{p}, \mathbf{q})$-torus knot in $S^{3}$. Precisely for this class of knots, Lawrence and Rozansky [74] have again observed on the basis of empirical formulae generalizing (1.7) and (1.8) that the stationary-phase approximation to the Wilson loop path integral is exact. So like our previous work in [18], one very specific motivation for the present paper is to offer a theoretical explanation of the remarkable results in [74].

The special nature of the Seifert loop operators in $M$ has been also pointed out by Aganagic et al. [2], who consider such Wilson loops and others in the context of $q$-deformed Yang-Mills theory on the Riemann surface $\Sigma$. See $[2,3,8,20,27,28,31-36,61,67]$ for a variety of additional papers which discuss Chern-Simons theory on a Seifert manifold and the closely related subject of $q$-deformed Yang-Mills theory on a Riemann surface. We also mention attempts by Hahn [57] to make sense of the Wilson loop path integral in Chern-Simons theory in a more formal mathematical framework. See [103] for other work in this direction. Finally, we refer the interested reader to the very beautiful and roughly analogous work by Pestun [93] on path integral localization for supersymmetric circular Wilson loop operators in four-dimensional Yang-Mills theory. See also the work of Kapustin and collaborators [75] (which has a certain degree of overlap with this paper) for a more recent application of Pestun's techniques to Wilson loops in superconformal Chern-Simons theories. Last but not least, related ideas have been pursued by Nekrasov et al. in $[17,88]$.

\subsection{The plan of the paper}

In order to apply non-abelian localization to the general Seifert loop path integral in (1.5), we must first recast this path integral as a symplectic integral of the canonical form (1.3). Although not immediately obvious, the symplectic description of the Seifert loop path integral turns out to be a wonderfully natural extension of our prior results for the Chern-Simons path integral in (1.1). To make this fact apparent and also for sake of readability, we have endeavored to keep the presentation self-contained - a goal which at least partially accounts for the length of the present paper.

With the ambition above in mind, we begin in Section 2 by quickly recalling how the path integral of Yang-Mills theory on a Riemann surface 
$\Sigma$ furnishes the basic example of a symplectic integral of the canonical form (1.3). Along the way, we establish some standard notation and conventions for the paper.

Next, in Section 3 we review how the path integral which describes the partition function of Chern-Simons theory on a Seifert manifold can also be put into the canonical symplectic form. The material here closely follows Section 3 of [18].

Finally, in Section 4 we generalize the results in Section 3 to the Seifert loop path integral.

At the heart of Section 4 lies a very old and very general piece of gauge theory lore, which is perhaps worth mentioning now. As suggested by Witten in one of the small gems of [105], if we are to analyze a Wilson loop expectation value semi-classically, we must use a corresponding semi-classical description for the Wilson loop operator itself. That is, rather than applying the conventional definition of $W_{R}(C)$ in (1.4), we represent the Wilson loop operator by a path integral over an auxiliary bosonic field $U$ attached to the curve $C$ and coupled to the restriction of $A$ to $C$. In these terms, we schematically write

$$
W_{R}(C)=\int \mathcal{D} U \exp \left[\operatorname{ics}\left(U ;\left.A\right|_{C}\right)\right] .
$$

A bit more precisely, the field $U$ describes a one-dimensional sigma model on $C$ whose target space is the coadjoint orbit $\mathcal{O}_{\alpha}$ of $G$, which passes through the highest weight $\alpha$ of $R$ and $\operatorname{cs}_{\alpha}\left(U ;\left.A\right|_{C}\right)$ is a local, gauge-invariant, and indeed topological action that specifies the coupling of $U$ to the background gauge field $A$. Since the semi-classical description (1.11) of $W_{R}(C)$ proves to be an essential ingredient for our analysis, we review this description in detail in Section 4.

Although Chern-Simons theory might seem to be characterized as an intrinsically three-dimensional gauge theory, an important outcome of our work in both Sections 3 and 4 is to provide a general reformulation of ChernSimons theory on an arbitrary three-manifold $M$ in such a way that one of the three components of the connection $A$ completely decouples. This construction fundamentally underlies our work on non-abelian localization, but it may have other applications as well. At the moment, one tantalizing geometric aspect of this reformulation of Chern-Simons theory is that it relies upon the choice of a contact structure on $M$.

Although perhaps anticlimactic, in Section 5 we return to two dimensions and consider the analogue for the Seifert loop operator in Yang-Mills theory 
on $\Sigma$. As pointed out long ago by Witten [106], the two-dimensional analogue of the Seifert loop operator is a local "monodromy" operator which inserts a classical singularity into the gauge field $A$ at a marked point of $\Sigma$. Like the Seifert loop operators, the monodromy operators in two-dimensional Yang-Mills theory are described by a path integral of the canonical symplectic form (1.3). This beautiful observation, which we review in some detail, can be traced back to remarks of Atiyah in Section 5.2 of [10]. The monodromy operators in two-dimensional Yang-Mills theory are also related to the surface operators considered more recently by Gukov and Witten [70] in the context of four-dimensional gauge theory. Indeed, portions of the exposition in Section 5 have a basic overlap with material in [70].

In preparation for actual computations, we discuss in Section 6 general aspects of non-abelian localization. In particular, we explain how nonabelian localization provides a cohomological interpretation for symplectic integrals such as (1.3), and we recall a general non-abelian localization formula derived in [18]. Thankfully, this localization formula is again applicable, so we do not need to extend the lengthy technical analysis of [18]. At the end of Section 6, we also review two elementary, finite-dimensional examples of non-abelian localization.

Finally, in Section 7 we perform explicit computations of Seifert loop path integrals using non-abelian localization. As in our previous study of the Chern-Simons partition function, we focus on two extreme cases.

First, in the case that $M$ is a Seifert homology sphere, we evaluate the local contribution from the trivial connection to the Seifert loop path integral. Because the first homology group of $M$ is by assumption zero, $H_{1}(M ; \mathbb{Z})=0$, the trivial connection is an isolated flat connection. However, the trivial connection is also fixed by constant gauge transformations on $M$. So just as in [18], the contribution of the trivial connection to the Seifert loop path integral reduces to an integral over the Cartan subalgebra $t$ of the gauge group $G$ itself. As we found experimentally in (1.8), the Seifert loop operator for a given irreducible representation $R$ of $G$ then appears in the integral over $\mathfrak{t}$ as the corresponding character $\mathrm{ch}_{R}$.

Among other results, we thus provide an exact path integral calculation for the expectation value of a Wilson loop operator wrapping an arbitrary torus knot in $S^{3}$. As a special case, for gauge group $G=S U(2)$ we recover the well-known expression for the Jones polynomial of a torus knot. Another satisfying aspect of this calculation is that we observe the renowned Weyl character formula to emerge naturally from the Chern-Simons path integral, in a manner reminiscent of its classic derivation by Atiyah and Bott [15] from index theory. 
At the opposite extreme, in the case that $M$ is a smooth circle bundle over a Riemann surface $\Sigma$ of genus $h \geq 1$, we compute the cohomology class that represents the Seifert loop operator on a smooth component of the moduli space of flat connections on $M$. Such a component consists of irreducible connections, whose stabilizers in the group of all gauge transformations arise solely from the center of $G$. In this setting, the cohomology class that describes the Seifert loop operator turns out to be extremely natural. Namely, the Seifert loop class is given by the Chern character $^{2}$ of a universal bundle associated to the representation $R$. In fact, the Seifert loop class already appears in related work of Teleman and Woodward [100,101], where it derives from a tautological Atiyah-Bott generator in the $K$-theory of the moduli stack of holomorphic bundles over $\Sigma$. As we discuss, our computation of the Seifert loop class is also strongly suggested by prior work of Jeffrey [65] on the Verlinde formula.

For the convenience of the reader, we include in Appendix A an index of commonly used notation. The remaining appendices contain a few technical calculations associated to our work in Section 7.

\section{The symplectic geometry of Yang-Mills theory on a Riemann surface}

A central theme throughout this work is the relationship between ChernSimons theory on a Seifert manifold $M$ and Yang-Mills theory on the associated Riemann surface $\Sigma$. Thus as a warm-up for our discussion of path integrals in Chern-Simons theory, let us quickly recall the much simpler symplectic interpretation for the path integral of two-dimensional YangMills theory.

We start by considering the usual path integral, which describes the partition function of Yang-Mills theory on a Riemann surface $\Sigma$,

$$
\begin{aligned}
Z(\epsilon) & =\frac{1}{\operatorname{Vol}(\mathcal{G}(P))}\left(\frac{1}{2 \pi \epsilon}\right)^{\Delta_{\mathcal{G}(P)} / 2} \int_{\mathcal{A}(P)} \mathcal{D} A \exp \left[\frac{1}{2 \epsilon} \int_{\Sigma} \operatorname{Tr}\left(F_{A} \wedge \star F_{A}\right)\right] \\
\Delta_{\mathcal{G}(P)} & =\operatorname{dim} \mathcal{G}(P) .
\end{aligned}
$$

Here we assume that the Yang-Mills gauge group $G$ is compact, connected, and simple. At times, especially for our discussion of Chern-Simons theory, we will further specialize to the case that $G$ is simply-connected as well.

\footnotetext{
${ }^{2}$ This description of the Seifert loop class was presciently suggested to me by E. Witten as I was in the midst of this work.
} 
"ATMP-17-1-A1-BEA" — 2013/5/15 - 19:32 — page $11-\# 11$

As is standard in gauge theory, we have introduced in (2.1) an invariant quadratic form ' $\mathrm{Tr}$ ' on the Lie algebra $\mathfrak{g}$ of $G$. Our conventions for the form ' $T r$ ' are as follows. If $G=S U(r+1)$, then ' $\mathrm{Tr}$ ' denotes the trace in the fundamental representation. Because the generators of the Lie algebra of $S U(r+1)$ are anti-Hermitian, the trace then determines a negative-definite quadratic form on the Lie algebra. For other simple Lie groups, ' $\mathrm{Tr}$ ' denotes the unique invariant, negative-definite quadratic form on $\mathfrak{g}$, which is normalized so that, for simply connected $G$, the Chern-Simons level $k$ in (1.1) obeys the conventional integral quantization.

With these conventions, the gauge field $A$ is anti-Hermitian, and the covariant derivative defined by $A$ is $d_{A}=d+A$. The curvature of $A$ is then $F_{A}=d A+A \wedge A$, as appears in the Yang-Mills action in (2.1). Of course, the parameter $\epsilon$ appearing there is related to the conventional Yang-Mills coupling $g_{\mathrm{ym}}$ via $\epsilon=g_{\mathrm{ym}}^{2}$.

In order to define $Z$ formally, we fix a principal $G$-bundle $P$ over $\Sigma$. Then the space $\mathcal{A}(P)$ over which we integrate in (2.1) is the space of connections on $P$. The group $\mathcal{G}(P)$ of gauge transformations acts on $\mathcal{A}(P)$, and we have normalized $Z$ in (2.1) by dividing by the volume of $\mathcal{G}(P)$ and a formal power of $\epsilon$. As we explained in [18], this normalization of $Z$ is the natural normalization when we apply non-abelian localization to compute the twodimensional Yang-Mills path integral.

The space $\mathcal{A}(P)$ is an affine space, which means that, if we choose a particular basepoint $A_{0}$ in $\mathcal{A}(P)$, then we can identify $\mathcal{A}(P)$ with its tangent space at $A_{0}$. This tangent space is the vector space of sections of the bundle $\Omega_{\Sigma}^{1} \otimes \operatorname{ad}(P)$ of one-forms on $\Sigma$ taking values in the adjoint bundle associated to $P$. In other words, an arbitrary connection $A$ on $P$ can be written as $A=A_{0}+\eta$ for some section $\eta$ of $\Omega_{\Sigma}^{1} \otimes \operatorname{ad}(P)$. As the space $\mathcal{A}(P)$ is affine, we define the path-integral measure $\mathcal{D} A$ up to an overall multiplicative constant by taking any translation-invariant measure on $\mathcal{A}(P)$.

To define the Yang-Mills action in (2.1), we must introduce a duality operator $\star$ on $\Sigma$. For two-dimensional Yang-Mills theory, we only require that the operator $\star$ relates zero-forms to two-forms, and to obtain such an operator we only need a symplectic structure, as opposed to a metric, on $\Sigma$. Given a symplectic form $\omega$ on $\Sigma$, we define $\star$ by the condition $\star 1=\omega$. The symplectic form $\omega$ is invariant under all area-preserving diffeomorphisms of $\Sigma$, and this large group acts as a symmetry of two-dimensional Yang-Mills theory. More precisely, this symmetry group is "large" in the sense that its complexification is the full group of orientation-preserving diffeomorphisms of $\Sigma$ [42]. This fact is fundamentally responsible for the topological nature of two-dimensional Yang-Mills theory. 
"ATMP-17-1-A1-BEA" — 2013/5/15 - 19:32 — page 12 — \#12

\subsection{The Yang-Mills path integral as a symplectic integral}

To interpret the two-dimensional Yang-Mills path integral as a symplectic integral of the canonical form in (1.3), we just need to identify the gauge theory counterparts of the abstract geometric data that enter the symplectic integral. These data consist of a symplectic manifold $X$ with symplectic form $\Omega$, a Lie group $H$ with Hamiltonian action on $X$, and an invariant quadratic form $(\cdot, \cdot)$ on the Lie algebra $\mathfrak{h}$ of $H$. The corresponding quantities in two-dimensional Yang-Mills theory are very easy to guess.

First, the affine space $\mathcal{A}(P)$ carries a natural symplectic form $\Omega$ determined by the intersection pairing on $\Sigma$ itself. Explicitly, if $\eta$ and $\xi$ are any two tangent vectors to $\mathcal{A}(P)$, represented on $\Sigma$ by sections of $\Omega_{\Sigma}^{1} \otimes \operatorname{ad}(P)$, then $\Omega$ is defined by

$$
\Omega(\eta, \xi)=-\int_{\Sigma} \operatorname{Tr}(\eta \wedge \xi) .
$$

Clearly $\Omega$ is closed, non-degenerate, and invariant under both gauge transformations and translations on $\mathcal{A}(P)$. Thus, $\mathcal{A}(P)$ plays the role of the abstract symplectic manifold $X$ in (1.3).

Similarly, we identify the translation-invariant path-integral measure $\mathcal{D} A$ with the symplectic measure on $\mathcal{A}(P)$ determined by $\Omega$. Quite generally, if $X$ is a symplectic manifold of dimension $2 n$ with symplectic form $\Omega$, then the symplectic measure on $X$ is given by the top-form $\Omega^{n} / n$ !. This measure can be represented uniformly for $X$ of arbitrary dimension by the expression $\exp (\Omega)$, where we implicitly pick out from the series expansion of the exponential the term which is of top degree on $X$. So we formally write $\mathcal{D} A=\exp (\Omega)$.

As for the Hamiltonian group $H$ acting on $X$, the obvious candidate for this role in two-dimensional Yang-Mills theory is the group $\mathcal{G}(P)$ of gauge transformations acting on $\mathcal{A}(P)$. Indeed, as was observed long ago by Atiyah and Bott [12], the action of $\mathcal{G}(P)$ on $\mathcal{A}(P)$ is Hamiltonian with respect to the symplectic form $\Omega$.

To recall what the Hamiltonian condition implies, we consider the general situation that a connected Lie group $H$ with Lie algebra $\mathfrak{h}$ acts on a symplectic manifold $X$ preserving the symplectic form $\Omega$. The action of $H$ on $X$ is then Hamiltonian when there exists an algebra homomorphism from $\mathfrak{h}$ to the algebra of functions on $X$ under the Poisson bracket. The Poisson bracket of functions $f$ and $g$ on $X$ is given by $\{f, g\}=-V_{f}(g)$, where $V_{f}$ is the Hamiltonian vector field associated to $f$. This vector field 
"ATMP-17-1-A1-BEA" — 2013/5/15 - 19:32 — page 13 — \#13

is determined by the relation $d f=\iota_{V_{f}} \Omega$, where $\iota_{V_{f}}$ is the interior product with $V_{f}$. More explicitly, in local canonical coordinates on $X$, the components of $V_{f}$ are determined by $f$ as $V_{f}^{m}=-\left(\Omega^{-1}\right)^{m n} \partial_{n} f$, where $\Omega^{-1}$ is an "inverse" to $\Omega$ that arises by considering the symplectic form as an isomorphism $\Omega: T M \rightarrow T^{*} M$ with inverse $\Omega^{-1}: T^{*} M \rightarrow T M$. In coordinates, $\Omega^{-1}$ is defined by $\left(\Omega^{-1}\right)^{l m} \Omega_{m n}=\delta_{n}^{l}$, and $\{f, g\}=\Omega_{m n} V_{f}^{m} V_{g}^{n}$.

The algebra homomorphism from the Lie algebra $\mathfrak{h}$ to the algebra of functions on $X$ under the Poisson bracket is then specified by a moment map $\mu: X \rightarrow \mathfrak{h}^{*}$, under which an element $\phi$ of $\mathfrak{h}$ is sent to the function $\langle\mu, \phi\rangle$ on $X$, where $\langle\cdot, \cdot\rangle$ is the dual pairing between $\mathfrak{h}$ and $\mathfrak{h}^{*}$. More generally, we use $\langle\cdot, \cdot\rangle$ throughout this paper to denote the canonical pairing between any vector space and its dual. The moment map by definition satisfies the relation

$$
d\langle\mu, \phi\rangle=\iota_{V(\phi)} \Omega,
$$

where $V(\phi)$ is the vector field on $X$, which is generated by the infinitesimal action of $\phi$. In terms of $\mu$, the Hamiltonian condition then becomes the condition that $\mu$ also satisfy

$$
\{\langle\mu, \phi\rangle,\langle\mu, \psi\rangle\}=\langle\mu,[\phi, \psi]\rangle .
$$

Geometrically, equation (2.4) is an infinitesimal expression of the condition that the moment map $\mu$ commute with the action of $H$ on $X$ and the coadjoint action of $H$ on $\mathfrak{h}^{*}$.

Returning from this abstract discussion to the case of Yang-Mills theory on $\Sigma$, let us consider the moment map for the action of $\mathcal{G}(P)$ on $\mathcal{A}(P)$. If $\phi$ is an element of the Lie algebra of $\mathcal{G}(P)$ and hence is represented on $\Sigma$ by a section of $\operatorname{ad}(P)$, the corresponding vector field $V(\phi)$ on $\mathcal{A}(P)$ is given as usual by ${ }^{3}$

$$
V(\phi)=-d_{A} \phi, \quad d_{A} \phi=d \phi+[A, \phi] .
$$

We then compute directly using (2.2),

$$
\iota_{V(\phi)} \Omega=\int_{\Sigma} \operatorname{Tr}\left(d_{A} \phi \wedge \delta A\right)=-\int_{\Sigma} \operatorname{Tr}\left(\phi d_{A} \delta A\right)=-\delta \int_{\Sigma} \operatorname{Tr}\left(F_{A} \phi\right) .
$$

\footnotetext{
${ }^{3}$ In order to make some conventions for our discussion of Wilson loops more natural, we have made the opposite choice for the sign of $V(\phi)$ as compared to that in [18]. As a result, certain formulae in this paper differ by signs from the corresponding expressions in [18].
} 
Here, we write $\delta$ for the exterior derivative acting on $\mathcal{A}(P)$, so that, for instance, $\delta A$ is regarded as a one form on $\mathcal{A}(P)$. Thus, the relation $(2.3)$ determines, up to an additive constant, that the moment map $\mu$ for the action of $\mathcal{G}(P)$ on $\mathcal{A}(P)$ is given by

$$
\langle\mu, \phi\rangle=-\int_{\Sigma} \operatorname{Tr}\left(F_{A} \phi\right)
$$

One can then check directly that $\mu$ in (2.7) satisfies the condition (2.4) that it arise from a Lie algebra homomorphism, and this condition fixes the arbitrary additive constant that could otherwise appear in $\mu$ to be zero.

Thus $\mathcal{G}(P)$ acts in a Hamiltonian fashion on $\mathcal{A}(P)$ with moment map $\mu=F_{A}$. Here, we regard the curvature $F_{A}$, transforming on $\Sigma$ as a section of $\Omega_{\Sigma}^{2} \otimes \operatorname{ad}(P)$, more abstractly as an element of the dual of the Lie algebra of $\mathcal{G}(P)$.

Finally, to define the canonical symplectic integral in (1.3), the Lie algebra $\mathfrak{h}$ of the Hamiltonian group $H$ must carry an invariant quadratic form $(\cdot, \cdot)$, which we use to define the invariant function $S=\frac{1}{2}(\mu, \mu)$. In the case of Yang-Mills theory on $\Sigma$, we use the duality operator $\star$ and the invariant form ' $\mathrm{Tr}$ ' to introduce the obvious positive-definite, invariant quadratic form on the Lie algebra of $\mathcal{G}(P)$, given explicitly by

$$
(\phi, \phi)=-\int_{\Sigma} \operatorname{Tr}(\phi \wedge \star \phi) .
$$

We formally define the volume of $\mathcal{G}(P)$ as appears in (2.1) using the quadratic form (2.8). With respect to this quadratic form, the Yang-Mills action is precisely the square of the moment map in (2.7),

$$
S=\frac{1}{2}(\mu, \mu)=-\frac{1}{2} \int_{\Sigma} \operatorname{Tr}\left(F_{A} \wedge \star F_{A}\right) .
$$

As a result, the path integral (2.1) of Yang-Mills theory on $\Sigma$ can be recast completely in terms of the symplectic data associated to the Hamiltonian action of $\mathcal{G}(P)$ on $\mathcal{A}(P)$,

$$
Z(\epsilon)=\frac{1}{\operatorname{Vol}(\mathcal{G}(P))}\left(\frac{1}{2 \pi \epsilon}\right)^{\Delta_{\mathcal{G}(P)} / 2} \int_{\mathcal{A}(P)} \exp \left[\Omega-\frac{1}{2 \epsilon}(\mu, \mu)\right]
$$

just as in (1.3). 
"ATMP-17-1-A1-BEA" — 2013/5/15 - 19:32 — page 15 — \#15

\section{The symplectic geometry of Chern-Simons theory on a Seifert manifold}

Following Section 3 of [18], our goal in this section is to review how the path integral which describes the partition function of Chern-Simons theory on a Seifert manifold can be recast as a symplectic integral of the canonical form (1.3). For the convenience of the reader, the treatment below is both self-contained and reasonably complete, though not so exhaustive as that in $[18]$.

To setup notation, we consider Chern-Simons gauge theory on a threemanifold $M$ with compact, connected, simply connected, and simple gauge group $G$. With these assumptions, any principal $G$-bundle $P$ on $M$ is topologically trivial, and we denote by $\mathcal{A}$ the affine space of connections on the trivial bundle. We denote by $\mathcal{G}$ the group of gauge transformations acting on $\mathcal{A}$.

We begin with the Chern-Simons path integral

$$
\begin{aligned}
Z(\epsilon) & =\frac{1}{\operatorname{Vol}(\mathcal{G})}\left(\frac{1}{2 \pi \epsilon}\right)^{\Delta_{\mathcal{G}}} \int_{\mathcal{A}} \mathcal{D} A \exp \left[\frac{\mathrm{i}}{2 \epsilon} \int_{M} \operatorname{Tr}\left(A \wedge d A+\frac{2}{3} A \wedge A \wedge A\right)\right] \\
\epsilon & =\frac{2 \pi}{k}, \quad \Delta_{\mathcal{G}}=\operatorname{dim} \mathcal{G} .
\end{aligned}
$$

Here, we have introduced a coupling parameter $\epsilon$ by analogy to the canonical symplectic integral in (1.3), and we have included a number of formal factors in $Z$. First, we have the measure $\mathcal{D} A$ on $\mathcal{A}$, which we again define up to norm as a translation-invariant measure on $\mathcal{A}$. As usual, we have also divided the path integral by the volume of the group of gauge transformations $\mathcal{G}$. Finally, to be fastidious, we have normalized $Z$ by a formal power of $\epsilon$ which as in (2.1) is natural when $Z$ is defined by localization.

At the moment, we make no assumption about the three-manifold $M$. However, if $M$ is a Seifert manifold, then to interpret the Chern-Simons path integral symplectically we must eventually decouple one of the three components of the gauge field $A$. This observation motivates the following reformulation of Chern-Simons theory, which proves to be key to the rest of the paper.

\subsection{A new formulation of Chern-Simons theory, part I}

In order to decouple one of the components of $A$, we begin by choosing a one-dimensional subbundle of the cotangent bundle $T^{*} M$ of $M$. Locally on 
$M$, this choice can be represented by the choice of an everywhere non-zero one-form $\kappa$, so that the subbundle of $T^{*} M$ consists of all one-forms proportional to $\kappa$. However, if $t$ is any non-zero function, then clearly $\kappa$ and $t \kappa$ generate the same subbundle in $T^{*} M$. Thus, our choice of a one-dimensional subbundle of $T^{*} M$ corresponds locally to the choice of an equivalence class of one-forms under the relation

$$
\kappa \sim t \kappa .
$$

We note that the representative one-form $\kappa$ which generates the subbundle need only be defined locally on $M$. Globally, the subbundle might or might not be generated by a non-zero one-form, which is defined everywhere on $M$; this condition depends upon whether the sign of $\kappa$ can be consistently defined under (3.2) and thus whether the subbundle is orientable or not.

We now attempt to decouple one of the three components of $A$. Specifically, our goal is to reformulate Chern-Simons theory on $M$ as a theory that respects a new local symmetry under which $A$ varies as

$$
\delta A=\sigma \kappa .
$$

Here $\sigma$ is an arbitrary section of the bundle $\Omega_{M}^{0} \otimes \mathfrak{g}$ of Lie algebra-valued functions on $M$.

The Chern-Simons action certainly does not respect the local "shift" symmetry in (3.3). However, we can trivially introduce this shift symmetry into Chern-Simons theory if we simultaneously introduce a new scalar field $\Phi$ on $M$ which transforms like $A$ in the adjoint representation of the gauge group. Under the shift symmetry, $\Phi$ transforms as

$$
\delta \Phi=\sigma .
$$

For future reference, we denote the infinite-dimensional group of shift symmetries parameterized by $\sigma$ as $\mathcal{S}$.

Now, if $\kappa$ in (3.3) is scaled by a non-zero function $t$ so that $\kappa \rightarrow t \kappa$, then this rescaling can be absorbed into the arbitrary section $\sigma$, which also appears in (3.3), so that the transformation law for $A$ is well-defined. However, from the transformation (3.4) of $\Phi$ under the same symmetry, we see that because we absorb $t$ into $\sigma$ we must postulate an inverse scaling of $\Phi$, so that $\Phi \rightarrow t^{-1} \Phi$. As a result, although $\kappa$ is only locally defined up to scale, the product $\kappa \Phi$ is well defined on $M$.

The only extension of the Chern-Simons action that now incorporates both $\Phi$ and the shift symmetry is the Chern-Simons functional $\operatorname{CS}(\cdot)$ of 
"ATMP-17-1-A1-BEA" — 2013/5/15 — 19:32 — page 17 — \#17

the shift-invariant combination $A-\kappa \Phi$. Thus, we consider the theory with action

$$
S(A, \Phi)=\operatorname{CS}(A-\kappa \Phi)
$$

or more explicitly,

$$
S(A, \Phi)=\operatorname{CS}(A)-\int_{M}\left[2 \kappa \wedge \operatorname{Tr}\left(\Phi F_{A}\right)-\kappa \wedge d \kappa \operatorname{Tr}\left(\Phi^{2}\right)\right] .
$$

To proceed, we play the usual game used to derive field theory dualities by path integral manipulations, as for $T$-duality in two dimensions $[26,96]$ or abelian $S$-duality in four dimensions [104]. We have introduced a new degree of freedom, namely $\Phi$, into Chern-Simons theory, and we have simultaneously enlarged the symmetry group of the theory so that this degree of freedom is completely gauge trivial. As a result, we can either use the shift symmetry (3.4) to gauge $\Phi$ away, in which case we recover the usual description of Chern-Simons theory, or we can integrate $\Phi$ out, in which case we obtain a new description of Chern-Simons theory that respects the action of the shift symmetry (3.3) on $A$.

\subsubsection{A contact structure on $M$}

Hitherto, we have supposed that the one-dimensional subbundle of $T^{*} M$ represented by $\kappa$ is arbitrary, but at this point we must impose an important geometric condition on this subbundle. From the action $S(A, \Phi)$ in (3.6), we see that the term quadratic in $\Phi$ is multiplied by the local three-form $\kappa \wedge d \kappa$. In order for this quadratic term to be everywhere non-degenerate on $M$, so that we can easily perform the path integral over $\Phi$, we require that $\kappa \wedge d \kappa$ is also everywhere non-zero on $M$.

Although $\kappa$ itself is only defined locally and up to rescaling by a nonzero function $t$, the condition that $\kappa \wedge d \kappa \neq 0$ pointwise on $M$ is a globally well-defined condition on the subbundle generated by $\kappa$. For when $\kappa$ scales as $\kappa \rightarrow t \kappa$ for any non-zero function $t$, we easily see that $\kappa \wedge d \kappa$ also scales as $\kappa \wedge d \kappa \rightarrow t^{2} \kappa \wedge d \kappa$. Thus, the condition that $\kappa \wedge d \kappa \neq 0$ is preserved under arbitrary rescalings of $\kappa$.

The structure that we thus introduce on $M$ is the choice of a onedimensional subbundle of $T^{*} M$ for which any local generator $\kappa$ satisfies $\kappa \wedge d \kappa \neq 0$ at each point of $M$. This geometric structure, which appears so naturally here, is known as a contact structure $[19,48,54]$. More generally, on an arbitrary manifold $M$ of odd dimension $2 n+1$, a contact structure on $M$ is defined as a one-dimensional subbundle of $T^{*} M$ for which the local generator $\kappa$ satisfies $\kappa \wedge(d \kappa)^{n} \neq 0$ everywhere on $M$. 
In many ways, a contact structure is the analogue of a symplectic structure for manifolds of odd dimension. The fact that we must choose a contact structure on $M$ for our reformulation of Chern-Simons theory is thus closely related to the fact, mentioned previously, that we must choose a symplectic structure on the Riemann surface $\Sigma$ in order to define Yang-Mills theory on $\Sigma$.

We will say a bit more about contact structures on Seifert manifolds later, but for now, we just observe that, by a classic theorem of Martinet [82], any compact, orientable ${ }^{4}$ three-manifold possesses a contact structure.

\subsubsection{Path integral manipulations}

Without loss of generality, we choose a contact structure on the threemanifold $M$, and we consider the theory defined by the path integral

$$
\begin{aligned}
Z(\epsilon)= & \frac{1}{\operatorname{Vol}(\mathcal{G})} \frac{1}{\operatorname{Vol}(\mathcal{S})}\left(\frac{1}{2 \pi \epsilon}\right)^{\Delta_{\mathcal{G}}} \\
& \times \int \mathcal{D} A \mathcal{D} \Phi \exp \left[\frac{i}{2 \epsilon}\left(\operatorname{cs}(A)-\int_{M} 2 \kappa \wedge \operatorname{Tr}\left(\Phi F_{A}\right)+\int_{M} \kappa \wedge d \kappa \operatorname{Tr}\left(\Phi^{2}\right)\right)\right]
\end{aligned}
$$

Here the measure $\mathcal{D} \Phi$ is defined independently of any metric on $M$ by the invariant, positive-definite quadratic form

$$
(\Phi, \Phi)=-\int_{M} \kappa \wedge d \kappa \operatorname{Tr}\left(\Phi^{2}\right)
$$

which is invariant under the scaling $\kappa \rightarrow t \kappa, \Phi \rightarrow t^{-1} \Phi$. We similarly use this quadratic form to define formally the volume of the group $\mathcal{S}$ of shift symmetries, as appears in the normalization of (3.7).

Using the shift symmetry (3.4), we can fix $\Phi=0$ trivially, with unit Jacobian, and the resulting group integral over $\mathcal{S}$ produces a factor of $\operatorname{Vol}(\mathcal{S})$ to cancel the corresponding factor in the normalization of $Z(\epsilon)$. Hence, the new theory defined by (3.7) is fully equivalent to Chern-Simons theory.

On the other hand, because the field $\Phi$ appears only quadratically in the action (3.6), we can also perform the path integral over $\Phi$ directly. Upon

\footnotetext{
${ }^{4}$ We note that, because $\kappa \wedge d \kappa \rightarrow t^{2} \kappa \wedge d \kappa$ under a local rescaling of $\kappa$ and because $t^{2}$ is always positive, the sign of the local three-form $\kappa \wedge d \kappa$ is well-defined. So any threemanifold with a contact structure is necessarily orientable.
} 
"ATMP-17-1-A1-BEA" — 2013/5/15 - 19:32 — page 19 — \#19

integrating out $\Phi$, the new action $S(A)$ for the gauge field becomes

$$
S(A)=\int_{M} \operatorname{Tr}\left(A \wedge d A+\frac{2}{3} A \wedge A \wedge A\right)-\int_{M} \frac{1}{\kappa \wedge d \kappa} \operatorname{Tr}\left[\left(\kappa \wedge F_{A}\right)^{2}\right] .
$$

We find it convenient to abuse notation slightly by writing " $1 / \kappa \wedge d \kappa$ " in (3.9). To explain this notation precisely, we observe that, as $\kappa \wedge d \kappa$ is non-vanishing, we can always write $\kappa \wedge F_{A}=\varphi \kappa \wedge d \kappa$ for some function $\varphi$ on $M$ taking values in the Lie algebra $\mathfrak{g}$. Thus, we set $\kappa \wedge F_{A} / \kappa \wedge d \kappa=\varphi$, and the second term in $S(A)$ becomes $\int_{M} \kappa \wedge \operatorname{Tr}\left(F_{A} \varphi\right)$. As our notation in (3.9) suggests, this term is invariant under the transformation $\kappa \rightarrow t \kappa$, since $\varphi$ transforms as $\varphi \rightarrow t^{-1} \varphi$.

By construction, the new action $S(A)$ in (3.9) is invariant under the action of the shift symmetry (3.3) on $A$. Alternatively, one can directly check the shift-invariance of $S(A)$, for which one notes that the expression $\kappa \wedge F_{A}$ transforms under the shift symmetry as

$$
\kappa \wedge F_{A} \longrightarrow \kappa \wedge F_{A}+\sigma \kappa \wedge d \kappa .
$$

The partition function $Z(\epsilon)$ now takes the form

$$
\begin{aligned}
Z(\epsilon)= & \frac{1}{\operatorname{Vol}(\mathcal{G})} \frac{1}{\operatorname{Vol}(\mathcal{S})}\left(\frac{-\mathrm{i}}{2 \pi \epsilon}\right)^{\Delta_{\mathcal{G}} / 2} \\
& \times \int_{\mathcal{A}} \mathcal{D} A \exp \left[\frac { \mathrm { i } } { 2 \epsilon } \left(\int_{M} \operatorname{Tr}\left(A \wedge d A+\frac{2}{3} A \wedge A \wedge A\right)\right.\right. \\
& \left.\left.-\int_{M} \frac{1}{\kappa \wedge d \kappa} \operatorname{Tr}\left[\left(\kappa \wedge F_{A}\right)^{2}\right]\right)\right]
\end{aligned}
$$

where the Gaussian integral over $\Phi$ cancels some factors of $2 \pi \epsilon$ in the normalization of $Z$. As is standard, in integrating over $\Phi$ we assume that the integration contour has been slightly rotated off the real axis, effectively giving $\epsilon$ a small imaginary part, to regulate the oscillatory Gaussian integral. Thus, the theory described by the path integral (3.11) is fully equivalent to Chern-Simons theory, but now one component of $A$ manifestly decouples.

\subsection{The Chern-Simons path integral as a symplectic integral}

Our reformulation of the Chern-Simons partition function in(3.11) applies to any three-manifold $M$ with a specified contact structure. However, in order to apply non-abelian localization to Chern-Simons theory on $M$, we require that $M$ possesses additional symmetry. 
Specifically, we require that $M$ admits a locally free $U(1)$ action, which means that the generating vector field on $M$ associated to the infinitesimal action of $U(1)$ is nowhere vanishing. A free $U(1)$ action on $M$ clearly satisfies this condition, but more generally it is satisfied by any $U(1)$ action such that no point on $M$ is fixed by all of $U(1)$ (at such a point the generating vector field would vanish). Such an action need not be free, since some points on $M$ could be fixed by a cyclic subgroup of $U(1)$. The class of three-manifolds which admit a $U(1)$ action of this sort are precisely the Seifert manifolds.

To proceed further to a symplectic description of the Chern-Simons path integral, we now restrict attention to the case that $M$ is a Seifert manifold. We first review a few basic facts about such manifolds, for which a complete reference is [90].

\subsubsection{Contact structures on Seifert manifolds}

For simplicity, we begin by assuming that the three-manifold $M$ admits a free $U(1)$ action. In this case, $M$ is the total space of a circle bundle over a Riemann surface $\Sigma$,

$$
\begin{array}{r}
S^{1} \stackrel{n}{\longrightarrow} M \\
\\
\\
\stackrel{\downarrow}{\Sigma} \pi,
\end{array}
$$

and the free $U(1)$ action simply arises from rotations in the fiber of (3.12). The topology of $M$ is completely determined by the genus $h$ of $\Sigma$ and the degree $n$ of the bundle. Assuming that the bundle is non-trivial, we can always arrange by a suitable choice of orientation for $M$ that $n \geq 1$.

At this point, one might wonder why we restrict attention to the case of non-trivial bundles over $\Sigma$. As we now explain, in this case $M$ admits a natural contact structure that is invariant under the action of $U(1)$. As a result, our reformulation of Chern-Simons theory in (3.11) still respects this crucial symmetry of $M$.

To describe the $U(1)$ invariant contact structure on $M$, we simply exhibit an invariant one-form $\kappa$, defined globally on $M$, which satisfies the contact condition that $\kappa \wedge d \kappa$ is nowhere vanishing. To describe $\kappa$, we begin by choosing a symplectic form $\omega$ on $\Sigma$, which is normalized so that

$$
\int_{\Sigma} \omega=1
$$

Regarding $M$ as the total space of a principal $U(1)$-bundle, we take $\kappa$ to be a connection on this bundle (and hence a real-valued one-form on $M$ ) whose 
"ATMP-17-1-A1-BEA" — 2013/5/15 - 19:32 — page $21-\# 21$

curvature satisfies

$$
d \kappa=n \pi^{*} \omega
$$

where we recall that $n \geq 1$ is the degree of the bundle. For a nice, explicit description of $\kappa$ in this situation, see the description of the angular form in Section 6 of [25].

We let $\mathrm{R}$ (for "rotation") be the non-vanishing vector field on $M$, which generates the $U(1)$ action and which is normalized, so that its orbits have unit period. By the fundamental properties of a connection, $\kappa$ is invariant under the $U(1)$ action and satisfies $\langle\kappa, R\rangle=1$. Here $\langle\cdot, \cdot\rangle$ again denotes the canonical dual pairing. Thus, $\kappa$ pulls back to a non-zero one-form that generates the integral cohomology of each $S^{1}$ fiber of $M$, and we immediately see from (3.14) that $\kappa \wedge d \kappa$ is everywhere non-vanishing on $M$ so long as the bundle is non-trivial.

Of course, in the above construction we have assumed that $M$ admits a free $U(1)$ action, which is a more stringent requirement than the condition that no point of $M$ is completely fixed by the $U(1)$ action. However, an arbitrary Seifert manifold does admit an orbifold description precisely analogous to the description of $M$ as a principal $U(1)$-bundle over a Riemann surface. We simply replace the smooth Riemann surface $\Sigma$ with an orbifold $\widehat{\Sigma}$, and we replace the principal $U(1)$-bundle over $\Sigma$ with its orbifold counterpart, in such a way that the total space is a smooth three-manifold.

Concretely, the orbifold base $\widehat{\Sigma}$ of $M$ is now described by a Riemann surface of genus $h$ with marked points $p_{j}$ for $j=1, \ldots, N$ at which the coordinate neighborhoods are modeled not on $\mathbb{C}$ but on $\mathbb{C} / \mathbb{Z}_{a_{j}}$ for some cyclic group $\mathbb{Z}_{a_{j}}$, which acts on the local coordinate $z$ at $p_{j}$ as

$$
z \mapsto \zeta \cdot z, \quad \zeta=\mathrm{e}^{2 \pi \mathrm{i} / a_{j}}
$$

The choice of the particular orbifold points $p_{j}$ is topologically irrelevant, and the orbifold base $\widehat{\Sigma}$ can be completely specified by the genus $h$ and the set of integers $\left\{a_{1}, \ldots, a_{N}\right\}$.

We now consider a line $V$-bundle over $\widehat{\Sigma}$. Such an object is precisely analogous to a complex line bundle, except that the local trivialization over each orbifold point $p_{j}$ of $\widehat{\Sigma}$ is now modeled on $\mathbb{C} \times \mathbb{C} / \mathbb{Z}_{a_{j}}$, where $\mathbb{Z}_{a_{j}}$ acts on the local coordinates $(z, s)$ of the base and fiber as

$$
z \mapsto \zeta \cdot z, \quad s \mapsto \zeta^{b_{j}} \cdot s, \quad \zeta=\mathrm{e}^{2 \pi \mathrm{i} / a_{j}},
$$


for some integers $0 \leq b_{j}<a_{j}$.

Given such a line $V$-bundle over $\widehat{\Sigma}$, an arbitrary Seifert manifold $M$ can be described as the total space of the associated $S^{1}$ fibration. Of course, we require that $M$ itself be smooth. This condition implies that each pair of integers $\left(a_{j}, b_{j}\right)$ above must be relatively-prime, so that the local action (3.16) of the orbifold group $\mathbb{Z}_{a_{j}}$ on $\mathbb{C} \times S^{1}$ is free. In particular, we require $b_{j} \neq 0$ above.

The $U(1)$ action on $M$ again arises from rotations in the fibers over $\widehat{\Sigma}$, but this action is no longer free. Rather, the points in the $S^{1}$ fiber over each ramification point $p_{j}$ of $\widehat{\Sigma}$ are fixed by the cyclic subgroup $\mathbb{Z}_{a_{j}}$ of $U(1)$, due to the orbifold identification in (3.16).

Once the integers $\left\{b_{1}, \ldots, b_{N}\right\}$ are fixed, the topological isomorphism class of a line $V$-bundle on $\widehat{\Sigma}$ is specified by a single integer $n$, the degree. Thus, in total, the description of an arbitrary Seifert manifold $M$ is given by the Seifert invariants

$$
\left[h ; n ;\left(a_{1}, b_{1}\right), \ldots,\left(a_{N}, b_{N}\right)\right], \quad \operatorname{gcd}\left(a_{j}, b_{j}\right)=1 .
$$

As the basic notions of bundles, connections, curvatures, and (rational) characteristic classes generalize immediately from smooth manifolds to orbifolds [97,98], our previous construction of an invariant contact form $\kappa$ as a connection on a principal $U(1)$-bundle immediately generalizes to the orbifold situation here. In the orbifold case, if $\widehat{\mathcal{L}}$ denotes the line $V$-bundle over $\widehat{\Sigma}$ which describes $M$ with Seifert invariants (3.17), the Chern class of $\widehat{\mathcal{L}}$ is given by

$$
c_{1}(\widehat{\mathcal{L}})=n+\sum_{j=1}^{N} \frac{b_{j}}{a_{j}} .
$$

So long as $c_{1}(\widehat{\mathcal{L}})$ is non-zero (and positive by convention), then $\widehat{\mathcal{L}}$ is nontrivial, generalizing the previous condition that $n \geq 1$. In particular, $n$ can now be any integer such that $c_{1}(\widehat{\mathcal{L}})>0$. To define a contact structure on $M$ by analogy to (3.14), we then choose the connection $\kappa$, so that its curvature is given by

$$
d \kappa=\left(n+\sum_{j=1}^{N} \frac{b_{j}}{a_{j}}\right) \pi^{*} \widehat{\omega},
$$

where $\widehat{\omega}$ is a symplectic form on $\widehat{\Sigma}$ of unit volume, as in (3.13). 
In the course of our discussion, we have distinguished the orbifold $\widehat{\Sigma}$ from the smooth Riemann surface $\Sigma$. In the future, we will not make this artificial distinction, and for our discussion of Chern-Simons theory, we use $\Sigma$ to denote an arbitrary Riemann surface, possibly with orbifold points.

\subsubsection{A symplectic structure for Chern-Simons theory}

We now specialize to the case of Chern-Simons theory on a Seifert manifold $M$, which carries a distinguished $U(1)$ action and an invariant contact form $\kappa$. Our first task is to identify the symplectic space in Chern-Simons theory on $M$, which is to play the role of $X$ in the canonical symplectic integral (1.3).

Initially, the path integral of Chern-Simons theory is an integral over the affine space $\mathcal{A}$ of all connections on $M$, and unlike the case for twodimensional Yang-Mills theory, $\mathcal{A}$ is not naturally symplectic. However, we now reap the reward of our reformulation of Chern-Simons theory to decouple one component of $A$. Specifically, we consider the following twoform $\Omega$ on $\mathcal{A}$. If $\eta$ and $\xi$ are any two tangent vectors to $\mathcal{A}$, and hence are represented by sections of the bundle $\Omega_{M}^{1} \otimes \mathfrak{g}$ on $M$, we define $\Omega$ by

$$
\Omega(\eta, \xi)=-\int_{M} \kappa \wedge \operatorname{Tr}(\eta \wedge \xi)
$$

Because $\kappa$ is a globally defined one-form on $M$, the expression for $\Omega$ in (3.20) is also well defined. Further, $\Omega$ is manifestly closed and invariant under all symmetries. In particular, $\Omega$ is invariant under the group $\mathcal{S}$ of shift symmetries, and by virtue of this shift invariance, $\Omega$ is degenerate along tangent vectors to $\mathcal{A}$ of the form $\sigma \kappa$, where $\sigma$ is an arbitrary section of $\Omega_{M}^{0} \otimes \mathfrak{g}$.

Unlike the gauge symmetry $\mathcal{G}$, which acts non-linearly on $\mathcal{A}$, the shift symmetry $\mathcal{S}$ acts in a simple, linear fashion on $\mathcal{A}$. Thus we can trivially take the quotient of $\mathcal{A}$ by the action of $\mathcal{S}$, which we denote as

$$
\overline{\mathcal{A}}=\mathcal{A} / \mathcal{S} .
$$

Under this quotient, the pre-symplectic form $\Omega$ on $\mathcal{A}$ descends immediately to a symplectic form on $\overline{\mathcal{A}}$, which becomes a symplectic space naturally associated to Chern-Simons theory on $M$. So, $\overline{\mathcal{A}}$ plays the role of the abstract symplectic manifold $X$ in (1.3).

Our reformulation of the Chern-Simons action $S(A)$ in (3.9) is invariant under the shift symmetry $\mathcal{S}$, so $S(A)$ immediately descends to the quotient 
$\overline{\mathcal{A}}$. However, we should also think (at least formally) about the path integral measure $\mathcal{D} A$.

As we explained in some detail in Section 3.3 of [18], the translationinvariant measure $\mathcal{D} A$ on $\mathcal{A}$ pushes down to the symplectic measure defined by $\Omega$ on $\overline{\mathcal{A}}$. Along the way, the formal integral over the orbits of $\mathcal{S}$ contributes a factor of the volume $\operatorname{Vol}(\mathcal{S})$ to cancel the prefactor in $(3.11)$. Consequently, the Chern-Simons path integral reduces to the following integral over $\overline{\mathcal{A}}$,

$$
Z(\epsilon)=\frac{1}{\operatorname{Vol}(\mathcal{G})}\left(\frac{-\mathrm{i}}{2 \pi \epsilon}\right)^{\Delta_{\mathcal{G}} / 2} \int_{\overline{\mathcal{A}}} \exp \left[\Omega+\frac{\mathrm{i}}{2 \epsilon} S(A)\right]
$$

with

$$
S(A)=\int_{M} \operatorname{Tr}\left(A \wedge d A+\frac{2}{3} A \wedge A \wedge A\right)-\int_{M} \frac{1}{\kappa \wedge d \kappa} \operatorname{Tr}\left[\left(\kappa \wedge F_{A}\right)^{2}\right] .
$$

\subsection{Hamiltonian symmetries}

To complete our symplectic description of the Chern-Simons path integral on $M$, we must show that the action $S(A)$ in (3.23) is the square of a moment map $\mu$ for the Hamiltonian action of some symmetry group $\mathcal{H}$ on the symplectic space $\overline{\mathcal{A}}$.

By analogy to the case of Yang-Mills theory on $\Sigma$, one might naively guess that the relevant symmetry group for Chern-Simons theory would also be the group $\mathcal{G}$ of gauge transformations. One can easily check that the action of $\mathcal{G}$ on $\mathcal{A}$ descends under the quotient to a well-defined action on $\overline{\mathcal{A}}$, and clearly the symplectic form $\Omega$ on $\overline{\mathcal{A}}$ is invariant under $\mathcal{G}$. However, one interesting aspect of non-abelian localization for Chern-Simons theory is the fact that the group $\mathcal{H}$ which we use for localization must be somewhat more complicated than $\mathcal{G}$ itself.

A trivial objection to using $\mathcal{G}$ for localization is that, by construction, the square of the moment map $\mu$ for any Hamiltonian action on $\overline{\mathcal{A}}$ defines an invariant function on $\overline{\mathcal{A}}$, but the action $S(A)$ is not invariant under the group $\mathcal{G}$. Instead, the action $S(A)$ is the sum of a manifestly gauge-invariant term and the usual Chern-Simons action, which shifts by integral multiples of $8 \pi^{2}$ under "large" gauge transformations, those not continuously connected to the identity in $\mathcal{G}$. 
"ATMP-17-1-A1-BEA" — 2013/5/15 - 19:32 — page $25-\# 25$

This trivial objection is easily overcome. We consider not the disconnected group $\mathcal{G}$ of all gauge transformations but only the identity component $\mathcal{G}_{0}$ of this group, under which $S(A)$ is invariant.

We now consider the action of $\mathcal{G}_{0}$ on $\overline{\mathcal{A}}$, and our first task is to determine the corresponding moment map $\mu$. If $\phi$ is an element of the Lie algebra of $\mathcal{G}_{0}$, described by a section of the bundle $\Omega_{M}^{0} \otimes \mathfrak{g}$ on $M$, then the corresponding vector field $V(\phi)$ generated by $\phi$ on $\mathcal{A}$ is given ${ }^{5}$ by $V(\phi)=-d_{A} \phi$. Thus, from our expression for the symplectic form $\Omega$ in (3.20) we see that

$$
\iota_{V(\phi)} \Omega=\int_{M} \kappa \wedge \operatorname{Tr}\left(d_{A} \phi \wedge \delta A\right) .
$$

Integrating by parts with respect to $d_{A}$, we can rewrite (3.24) in the form $\delta\langle\mu, \phi\rangle$, where

$$
\langle\mu, \phi\rangle=-\int_{M} \kappa \wedge \operatorname{Tr}\left(\phi F_{A}\right)+\int_{M} d \kappa \wedge \operatorname{Tr}\left(\phi\left(A-A_{0}\right)\right) .
$$

Here $A_{0}$ is an arbitrary connection, corresponding to a basepoint in $\mathcal{A}$, which we must choose so that the second term in (3.25) can be honestly interpreted as the integral of a differential form on $M$. In the case that the gauge group $G$ is simply connected, so that the principal $G$-bundle over $M$ is necessarily trivial, the choice of a basepoint connection $A_{0}$ corresponds geometrically to the choice of a trivialization for the bundle on $M$. We will say more about this choice momentarily, but we first observe that the expression for $\mu$ in (3.25) is invariant under the shift symmetry and immediately descends to a moment map for the action of $\mathcal{G}$ on $\overline{\mathcal{A}}$.

The fact that we must choose a basepoint $A_{0}$ in $\mathcal{A}$ to define the moment map is very important in the following, and it is fundamentally a reflection of the affine structure of $\mathcal{A}$. In general, an affine space is a space which can be identified with a vector space only after some basepoint is chosen to represent the origin. In the case at hand, once $A_{0}$ is chosen, we can identify $\mathcal{A}$ with the vector space of sections $\eta$ of the bundle $\Omega_{M}^{1} \otimes \mathfrak{g}$ on $M$, via $A=A_{0}+\eta$, as we used in (3.25). However, $\mathcal{A}$ is not naturally itself a vector space, since $\mathcal{A}$ does not intrinsically possess a distinguished origin. This statement corresponds to the geometric statement that, although our principal $G$-bundle on $M$ is trivial, it does not possess a canonical trivialization.

In terms of the moment map $\mu$, the choice of $A_{0}$ simply represents the possibility of adding an arbitrary constant to $\mu$. In general, our ability to add

\footnotetext{
${ }^{5}$ Once again, we warn the reader that our convention for the sign of $V(\phi)$ is opposite from that in [18].
} 
a constant to $\mu$ means that $\mu$ need not determine a Hamiltonian action of $\mathcal{G}_{0}$ on $\overline{\mathcal{A}}$. Indeed, as we show below, the action of $\mathcal{G}_{0}$ on $\overline{\mathcal{A}}$ is not Hamiltonian and we cannot simply use $\mathcal{G}_{0}$ to perform localization.

In order not to clutter the expressions below, we assume henceforth that we have fixed a trivialization of the $G$-bundle on $M$ and we simply set $A_{0}=0$.

To determine whether the action of $\mathcal{G}_{0}$ on $\overline{\mathcal{A}}$ is Hamiltonian, we must check the condition (2.4) that $\mu$ determine a homomorphism from the Lie algebra of $\mathcal{G}_{0}$ to the algebra of functions on $\overline{\mathcal{A}}$ under the Poisson bracket. So we directly compute

$$
\begin{aligned}
\{\langle\mu, \phi\rangle,\langle\mu, \psi\rangle\} & =\Omega\left(d_{A} \phi, d_{A} \psi\right)=-\int_{M} \kappa \wedge \operatorname{Tr}\left(d_{A} \phi \wedge d_{A} \psi\right) \\
& =-\int_{M} \kappa \wedge \operatorname{Tr}\left([\phi, \psi] F_{A}\right)-\int_{M} d \kappa \wedge \operatorname{Tr}\left(\phi d_{A} \psi\right) \\
& =\langle\mu,[\phi, \psi]\rangle-\int_{M} d \kappa \wedge \operatorname{Tr}(\phi d \psi)
\end{aligned}
$$

Thus, the failure of $\mu$ to determine an algebra homomorphism is measured by the cohomology class of the Lie algebra cocycle

$$
\begin{aligned}
c(\phi, \psi) & =\{\langle\mu, \phi\rangle,\langle\mu, \psi\rangle\}-\langle\mu,[\phi, \psi]\rangle, \\
& =-\int_{M} d \kappa \wedge \operatorname{Tr}(\phi d \psi)=-\int_{M} \kappa \wedge d \kappa \operatorname{Tr}\left(\phi £_{\mathrm{R}} \psi\right) .
\end{aligned}
$$

In the second line of (3.27), we have rewritten the cocycle more suggestively by using the Lie derivative $£_{\mathrm{R}}$ along the vector field $\mathrm{R}$ on $M$, which generates the $U(1)$ action. The class of this cocycle is not zero, and no Hamiltonian action on $\overline{\mathcal{A}}$ exists for the group $\mathcal{G}_{0}$.

\subsubsection{Some facts about loop groups}

The cocycle appearing in (3.27) has a very close relationship to a similar cocycle that arises in the theory of loop groups, and some well-known loop group constructions feature heavily in our study of Chern-Simons theory. We briefly review these ideas, for which a general reference is [94].

When $G$ is a finite-dimensional Lie group, we recall that the loop group $L G$ is defined as the group of smooth maps $\operatorname{Map}\left(S^{1}, G\right)$ from $S^{1}$ to $G$. Similarly, the Lie algebra $L \mathfrak{g}$ of $L G$ is the algebra $\operatorname{Map}\left(S^{1}, \mathfrak{g}\right)$ of smooth maps from $S^{1}$ to $\mathfrak{g}$. When $\mathfrak{g}$ is simple, then the Lie algebra $L \mathfrak{g}$ admits a 
unique, $G$-invariant cocycle up to scale, and this cocycle is directly analogous to the cocycle we discovered in (3.27). If $\phi$ and $\psi$ are elements in the Lie algebra $L \mathfrak{g}$, then this cocycle is defined by

$$
c(\phi, \psi)=-\int_{S^{1}} \operatorname{Tr}(\phi d \psi)=-\int_{S^{1}} d \tau \operatorname{Tr}\left(\phi £_{\mathrm{R}} \psi\right) .
$$

In passing to the last expression, we have by analogy to (3.27) introduced a unit-length parameter $\tau$ on $S^{1}$, so that $\int_{S^{1}} d \tau=1$, and we have introduced the dual vector field $\mathrm{R}=\partial / \partial \tau$ which generates rotations of $S^{1}$.

In general, if $\mathfrak{g}$ is any Lie algebra and $c$ is a non-trivial cocycle, then $c$ determines a corresponding central extension $\widetilde{\mathfrak{g}}$ of $\mathfrak{g}$,

$$
\mathbb{R} \longrightarrow \widetilde{\mathfrak{g}} \longrightarrow \mathfrak{g} .
$$

As a vector space, $\widetilde{\mathfrak{g}}=\mathfrak{g} \oplus \mathbb{R}$, and the Lie algebra of $\widetilde{\mathfrak{g}}$ is given by the bracket

$$
[(\phi, a),(\psi, b)]=([\phi, \psi], c(\phi, \psi)),
$$

where $\phi$ and $\psi$ are elements of $\mathfrak{g}$, and $a$ and $b$ are elements of $\mathbb{R}$.

In the case of the Lie algebra $L \mathfrak{g}$, the cocycle $c$ appearing in (3.28) consequently determines a central extension $\widetilde{L \mathfrak{g}}$ of $L \mathfrak{g}$. When $G$ is simply connected, the extension determined by $c$ or any integral multiple of $c$ lifts to a corresponding extension of $L G$ by $U(1)$,

$$
U(1)_{\mathrm{Z}} \longrightarrow \widetilde{L G} \longrightarrow L G
$$

Here we use the subscript ' $Z$ ' to distinguish the central $U(1)$ in $\widetilde{L G}$ from another $U(1)$ that will appear shortly. Topologically, the extension $\widetilde{L G}$ is the total space of the $S^{1}$ bundle over $L G$ whose Euler class is represented by the cocyle of the extension, interpreted as an invariant two-form on $L G$. The fact that the Euler class must be integral is responsible for the corresponding quantization condition on the cocycle of the extension.

When $\mathfrak{g}$ is simple, the algebra $L \mathfrak{g}$ has a non-degenerate, invariant inner product, which is unique up to scale and is given by

$$
(\phi, \psi)=-\int_{S^{1}} d \tau \operatorname{Tr}(\phi \psi) .
$$

On the other hand, the corresponding extension $\widetilde{L g}$ does not possess a non-degenerate, invariant inner product, since any element of $\widetilde{L g}$ can be 
expressed as a commutator, so that $[\widetilde{L \mathfrak{g}}, \widetilde{L \mathfrak{g}}]=\widetilde{L \mathfrak{g}}$, and the center of $\widetilde{L \mathfrak{g}}$ is necessarily orthogonal to every commutator under an invariant inner product.

However, we can also consider the semidirect product $U(1)_{\mathrm{R}} \ltimes \widetilde{L G}$. Here $U(1)_{\mathrm{R}}$ is the group acting on $S^{1}$ by rigid rotations, inducing a natural action on $\overline{L G}$ by which we define the product. The important observation about the group $U(1)_{\mathrm{R}} \ltimes \widetilde{L G}$ is that it does admit an invariant, non-degenerate inner product on its Lie algebra.

Explicitly, the Lie algebra of $U(1)_{\mathbb{R}} \ltimes \widetilde{L G}$ is identified with $\mathbb{R} \oplus \widetilde{L \mathfrak{g}}=$ $\mathbb{R} \oplus L \mathfrak{g} \oplus \mathbb{R}$ as a vector space, and the Lie algebra is given by the bracket

$$
[(p, \phi, a),(q, \psi, b)]=\left(0,[\phi, \psi]+p £_{\mathrm{R}} \psi-q £_{\mathrm{R}} \phi, c(\phi, \psi)\right),
$$

where $£_{R}$ is the Lie derivative with respect to the vector field $R$ generating rotations of $S^{1}$. We then consider the manifestly non-degenerate inner product on $\mathbb{R} \oplus \widetilde{L \mathfrak{g}}$ which is given by

$$
((p, \phi, a),(q, \psi, b))=-\int_{M} d \tau \operatorname{Tr}(\phi \psi)-p b-q a .
$$

One can directly check that this inner product is invariant under the adjoint action determined by (3.33). We note that although this inner product is non-degenerate, it is not positive-definite because of the last two terms in $(3.34)$.

\subsubsection{Extension to Chern-Simons theory}

We now return to our original problem, which is to find a Hamiltonian action of a group $\mathcal{H}$ on $\overline{\mathcal{A}}$ to use for localization. The natural guess to consider the identity component $\mathcal{G}_{0}$ of the gauge group does not work, because the cocycle $c$ in (3.27) obstructs the action of $\mathcal{G}_{0}$ on $\overline{\mathcal{A}}$ from being Hamiltonian.

However, motivated by the loop group constructions, we consider now the central extension $\widetilde{\mathcal{G}}_{0}$ of $\mathcal{G}_{0}$ by $U(1)$ which is determined by the cocycle $c$ in (3.27),

$$
U(1)_{\mathrm{Z}} \longrightarrow \widetilde{\mathcal{G}}_{0} \longrightarrow \mathcal{G}_{0}
$$

Again we use the subscript ' $Z$ ' to distinguish the central $U(1)_{Z}$ in $\widetilde{\mathcal{G}_{0}}$ from the geometric $U(1)_{\mathrm{R}}$ that acts on the Seifert manifold $M$.

We assume that the central $U(1)_{\text {z }}$ subgroup of $\widetilde{\mathcal{G}_{0}}$ acts trivially on $\overline{\mathcal{A}}$, so that the moment map for the central generator $(0, a)$ of the Lie algebra is 
"ATMP-17-1-A1-BEA" — 2013/5/15 - 19:32 — page 29 — \#29

constant. Then by construction, we see from (3.27) and (3.30) that the new moment map for the action of $\widetilde{\mathcal{G}}_{0}$ on $\overline{\mathcal{A}}$ is given by the sum

$$
\langle\mu,(\phi, a)\rangle=-\int_{M} \kappa \wedge \operatorname{Tr}\left(\phi F_{A}\right)+\int_{M} d \kappa \wedge \operatorname{Tr}(\phi A)+a,
$$

and this moment map does satisfy the Hamiltonian condition

$$
\{\langle\mu,(\phi, a)\rangle,\langle\mu,(\psi, b)\rangle\}=\langle\mu,[(\phi, a),(\psi, b)]\rangle .
$$

The action of the extended group $\widetilde{\mathcal{G}_{0}}$ on $\overline{\mathcal{A}}$ is thus Hamiltonian with moment map in (3.36).

But $\widetilde{\mathcal{G}}_{0}$ is still not the group $\mathcal{H}$ which we must use to perform non-abelian localization in Chern-Simons theory! In order to realize the action $S(A)$ as the square of the moment map $\mu$ for some Hamiltonian group action on $\overline{\mathcal{A}}$, the Lie algebra of the group must first possess a non-degenerate, invariant inner product. Just as for the loop group extension $\widetilde{L G}$, the group $\widetilde{\mathcal{G}_{0}}$ does not possess such an inner product.

However, we can elegantly remedy this problem, just as it was remedied for the loop group, by also considering the geometric action of $U(1)_{\mathrm{R}}$ on $M$. The $U(1)_{\mathrm{R}}$ action on $M$ induces an action of $U(1)_{\mathrm{R}}$ on $\widetilde{\mathcal{G}}_{0}$, so we consider the associated semidirect product $U(1)_{\mathrm{R}} \ltimes \widetilde{\mathcal{G}}_{0}$. A non-degenerate, invariant inner product on the Lie algebra of $U(1)_{\mathrm{R}} \ltimes \widetilde{\mathcal{G}}_{0}$ is given by

$$
((p, \phi, a),(q, \psi, b))=-\int_{M} \kappa \wedge d \kappa \operatorname{Tr}(\phi \psi)-p b-q a,
$$

in direct correspondence with (3.34). As for the loop group, this quadratic form is of indefinite signature, due to the hyperbolic form of the last two terms in (3.38).

Finally, the $U(1)_{\mathrm{R}}$ action on $M$ immediately induces a corresponding action on $\mathcal{A}$. Since the contact form $\kappa$ is invariant under this action, the induced $U(1)_{\mathrm{R}}$ action on $\mathcal{A}$ descends to a corresponding action on the quotient $\overline{\mathcal{A}}$. In general, the vector field upstairs on $\mathcal{A}$, which is generated by an arbitrary element $(p, \phi, a)$ of the Lie algebra of $U(1)_{\mathrm{R}} \ltimes \widetilde{\mathcal{G}}_{0}$ is then given by

$$
\delta A=-d_{A} \phi+p £_{\mathrm{R}} A,
$$


where we recall that $\mathrm{R}$ is the vector field on $M$ generating the action of $U(1)_{\mathrm{R}}$. Clearly the moment for the new generator $(p, 0,0)$ is given by

$$
\langle\mu,(p, 0,0)\rangle=-\frac{1}{2} p \int_{M} \kappa \wedge \operatorname{Tr}\left(£_{\mathrm{R}} A \wedge A\right) .
$$

This moment is manifestly invariant under the shift symmetry and descends to $\overline{\mathcal{A}}$.

In fact, the action of $U(1)_{\mathrm{R}} \ltimes \widetilde{\mathcal{G}_{0}}$ on $\overline{\mathcal{A}}$ is Hamiltonian, with moment map

$$
\begin{aligned}
\langle\mu,(p, \phi, a)\rangle= & -\frac{1}{2} p \int_{M} \kappa \wedge \operatorname{Tr}\left(£_{\mathrm{R}} A \wedge A\right)-\int_{M} \kappa \wedge \operatorname{Tr}\left(\phi F_{A}\right) \\
& +\int_{M} d \kappa \wedge \operatorname{Tr}(\phi A)+a .
\end{aligned}
$$

To check this statement, it suffices to compute $\{\langle\mu,(p, 0,0)\rangle,\langle\mu,(0, \psi, 0)\rangle\}$, which is the only non-trivial Poisson bracket that we have not already computed. Thus,

$$
\begin{aligned}
\{\langle\mu,(p, 0,0)\rangle,\langle\mu,(0, \psi, 0)\rangle\}= & \Omega\left(p £_{\mathrm{R}} A,-d_{A} \psi\right)=p \int_{M} \kappa \wedge \operatorname{Tr}\left(£_{\mathrm{R}} A \wedge d_{A} \psi\right), \\
= & -p \int_{M} \kappa \wedge \operatorname{Tr}\left(£_{\mathrm{R}} \psi F_{A}\right) \\
& +p \int_{M} d \kappa \wedge \operatorname{Tr}\left(£_{\mathrm{R}} \psi A\right) \\
= & \left\langle\mu,\left(0, p £_{\mathrm{R}} \psi, 0\right)\right\rangle,
\end{aligned}
$$

as required by the Lie bracket (3.33). So, we identify

$$
\mathcal{H}=U(1)_{\mathrm{R}} \ltimes \widetilde{\mathcal{G}}_{0}
$$

as the relevant group of Hamiltonian symmetries to use for localization in Chern-Simons theory.

\subsection{The shift-invariant action as the square of the moment map}

By construction, the square $(\mu, \mu)$ of the moment map $\mu$ in (3.41) for the Hamiltonian action of $\mathcal{H}$ on $\overline{\mathcal{A}}$ is a function on $\overline{\mathcal{A}}$ invariant under $\mathcal{H}$. The new Chern-Simons action $S(A)$ in (3.9) is also a function on $\overline{\mathcal{A}}$ invariant under $\mathcal{H}$. Given the high degree of symmetry, we certainly expect that $(\mu, \mu)$ and $S(A)$ agree up to normalization. We now check this fact and fix the relative normalization. 
From (3.38) and (3.41), we see immediately that

$$
(\mu, \mu)=\int_{M} \kappa \wedge \operatorname{Tr}\left(£_{\mathrm{R}} A \wedge A\right)-\int_{M} \kappa \wedge d \kappa \operatorname{Tr}\left[\left(\frac{\kappa \wedge F_{A}-d \kappa \wedge A}{\kappa \wedge d \kappa}\right)^{2}\right]
$$

Using the identity

$$
\iota_{\mathrm{R}} A=\frac{d \kappa \wedge A}{d \kappa \wedge \kappa}
$$

let us rewrite (3.44) as

$$
\begin{aligned}
(\mu, \mu)= & \int_{M} \kappa \wedge \operatorname{Tr}\left(£_{\mathrm{R}} A \wedge A\right)+2 \int_{M} \kappa \wedge \operatorname{Tr}\left[\left(\iota_{\mathrm{R}} A\right) F_{A}\right] \\
& -\int_{M} \kappa \wedge d \kappa \operatorname{Tr}\left[\left(\iota_{\mathrm{R}} A\right)^{2}\right]-\int_{M} \frac{1}{\kappa \wedge d \kappa} \operatorname{Tr}\left[\left(\kappa \wedge F_{A}\right)^{2}\right] .
\end{aligned}
$$

We also require a somewhat more baroque identity,

$$
\begin{aligned}
\operatorname{cs}(A)= & \int_{M} \operatorname{Tr}\left(A \wedge d A+\frac{2}{3} A \wedge A \wedge A\right), \\
= & \int_{M} \kappa \wedge \operatorname{Tr}\left(£_{\mathrm{R}} A \wedge A\right)+2 \int_{M} \kappa \wedge \operatorname{Tr}\left[\left(\iota_{\mathrm{R}} A\right) F_{A}\right] \\
& -\int_{M} \kappa \wedge d \kappa \operatorname{Tr}\left[\left(\iota_{\mathrm{R}} A\right)^{2}\right] .
\end{aligned}
$$

At first glance, the identity in (3.47) may not be immediately obvious, but it can be checked by elementary means. See Section 3.5 of [18], especially (3.56) and (3.58) therein ${ }^{6}$, for a very explicit demonstration of (3.47).

From (3.46) and (3.47), we then obtain the beautiful result,

$$
\begin{aligned}
(\mu, \mu) & =\operatorname{CS}(A)-\int_{M} \frac{1}{\kappa \wedge d \kappa} \operatorname{Tr}\left[\left(\kappa \wedge F_{A}\right)^{2}\right], \\
& =S(A) .
\end{aligned}
$$

\footnotetext{
${ }^{6} \mathrm{~A}$ slight discrepancy exists between the numbering of equations in the arXived and published versions of [18]. We refer throughout to the version of [18] on the electronic arXiv.
} 
So, we finally write the Chern-Simons partition function as a symplectic integral over $\overline{\mathcal{A}}$ of the canonical form,

$$
Z(\epsilon)=\frac{1}{\operatorname{Vol}(\mathcal{G})}\left(\frac{-\mathrm{i}}{2 \pi \epsilon}\right)^{\Delta_{\mathcal{G}} / 2} \int_{\overline{\mathcal{A}}} \exp \left[\Omega+\frac{\mathrm{i}}{2 \epsilon}(\mu, \mu)\right] .
$$

\section{On Wilson loops and Seifert loops in Chern-Simons theory}

Our goal is now to extend the results in Section 3 concerning the partition function of Chern-Simons theory to corresponding results for the expectation values of Wilson loop operators.

As in Section 3, we do not require the Seifert condition initially. So we consider Chern-Simons theory on an arbitrary three-manifold $M$, endowed with a contact structure represented locally by a one-form $\kappa$. We similarly consider a general Wilson loop operator

$$
W_{R}(C)=\operatorname{Tr}_{R} P \exp \left(-\oint_{C} A\right)
$$

where $C$ is an oriented closed curve smoothly embedded ${ }^{7}$ in $M$, and $R$ is an irreducible representation of the simply connected gauge group $G$.

Throughout this paper, we find it useful to characterize the representation $R$ in terms of its highest weight. So, we pick a decomposition of the set $\mathfrak{R}$ of roots of $G$ into positive and negative subsets, $\mathfrak{R}=\mathfrak{R}_{+} \cup \mathfrak{R}_{-}$. With respect to that decomposition, we then take $\alpha \geq 0$ to be the highest weight of $R$.

We begin with the Wilson loop path integral,

$$
\begin{aligned}
Z(\epsilon ; C, R)= & \frac{1}{\operatorname{Vol}(\mathcal{G})}\left(\frac{1}{2 \pi \epsilon}\right)^{\Delta_{\mathcal{G}}} \int \mathcal{D} A W_{R}(C) \\
& \times \exp \left[\frac{\mathrm{i}}{2 \epsilon} \int_{M} \operatorname{Tr}\left(A \wedge d A+\frac{2}{3} A \wedge A \wedge A\right)\right], \\
\epsilon= & \frac{2 \pi}{k}, \quad \Delta_{\mathcal{G}}=\operatorname{dim} \mathcal{G} .
\end{aligned}
$$

Here, we have been careful to normalize $Z(\epsilon ; C, R)$ precisely as we normalized the basic Chern-Simons path integral in (3.1).

\footnotetext{
${ }^{7}$ The condition that $C$ be smoothly embedded in $M$ is not strictly required to define $W_{R}(C)$ as a sensible operator in gauge theory. Indeed, the Wilson loop expectation value in Chern-Simons theory can be computed exactly even for the case that $C$ is an arbitrary closed graph [107] in $M$.
} 
"ATMP-17-1-A1-BEA" — 2013/5/15 — 19:32 — page 33 — \#33

\subsection{A semi-classical description of the Wilson loop operator}

This paper relies on only one good idea, to which we now come.

We clearly need a good idea, because a naive attempt to reapply the path integral manipulations of Section 3 to the Wilson loop path integral in (4.2) runs immediately aground. To illustrate the difficulty with the direct approach, let us consider the obvious way to rewrite the Wilson loop path integral in a shift-invariant form,

$$
\begin{aligned}
Z(\epsilon ; C, R)= & \frac{1}{\operatorname{Vol}(\mathcal{G})} \frac{1}{\operatorname{Vol}(\mathcal{S})}\left(\frac{1}{2 \pi \epsilon}\right)^{\Delta_{\mathcal{G}}} \int \mathcal{D} A \mathcal{D} \Phi \mathcal{W}_{R}(C) \\
& \times \exp \left[\frac{\mathrm{i}}{2 \epsilon} \operatorname{cs}(A-\kappa \Phi)\right] .
\end{aligned}
$$

Here $\mathcal{W}_{R}(C)$ denotes the generalized Wilson loop operator defined not using $A$ but using the shift-invariant combination $A-\kappa \Phi$, so that

$$
\mathcal{W}_{R}(C)=\operatorname{Tr}_{R} P \exp \left[-\oint_{C}(A-\kappa \Phi)\right]
$$

Exactly as for our discussion of the analogous path integral in (3.7), we can use the shift symmetry to fix $\Phi=0$, after which the path integral in (4.3) reduces trivially to the Wilson loop path integral in (4.2).

However, to learn something useful from (4.3) we need to perform the path integral over $\Phi$, and as it stands, this integral is not easy to do. As the generalized Wilson loop operator $\mathcal{W}_{R}(C)$ is expressed in (4.4) as a complicated, non-local functional of $\Phi$, the path integral over $\Phi$ in (4.3) is not a Gaussian integral that we can trivially evaluate as we did for the Chern-Simons path integral in (3.7).

Very concretely, if we expand the path-ordered exponential in (4.4), we immediately encounter an awkward series of multiple integrals over $C$ extending to arbitrary order in $\Phi$,

$$
\begin{aligned}
\mathcal{W}_{R}(C)= & \operatorname{dim} R+\frac{1}{2} \oint_{C \times C} \operatorname{Tr}_{R} P\left[(A-\kappa \Phi)(\tau) \cdot(A-\kappa \Phi)\left(\tau^{\prime}\right)\right] \\
& -\frac{1}{6} \oint_{C \times C \times C} \operatorname{Tr}_{R} P\left[(A-\kappa \Phi)(\tau) \cdot(A-\kappa \Phi)\left(\tau^{\prime}\right)\right. \\
& \left.\cdot(A-\kappa \Phi)\left(\tau^{\prime \prime}\right)\right]+\cdots
\end{aligned}
$$


Here, we have introduced separate parameters $\tau, \tau^{\prime}$, and $\tau^{\prime \prime}$ on $C$ to make the structure of the double and triple integrals over $C$ manifest, and we recall that the path-ordering symbol $P$ implies that the factors in the multiple integrals are ordered so that $\tau \geq \tau^{\prime} \geq \tau^{\prime \prime}$. We indicate similar terms of quartic and higher order by '... ${ }^{8}$ As is hopefully clear, any direct attempt $^{9}$ to perform the path integral over $\Phi$ in (4.3) would be painful, to say the least.

A more fundamental perspective on our problem is the following. Let us return to the description of the ordinary Wilson loop operator $W_{R}(C)$ as the trace in the representation $R$ of the holonomy of $A$ around $C$,

$$
W_{R}(C)=\operatorname{Tr}_{R} P \exp \left(-\oint_{C} A\right)
$$

As observed by Witten in Section 3.3 of [105], this description of $W_{R}(C)$ should be regarded as intrinsically quantum mechanical, for the simple reason that $W_{R}(C)$ can be naturally interpreted in (4.6) as the partition function of an auxiliary quantum system attached to the curve $C$. We will eventually make this interpretation very precise, but briefly, the representation $R$ is to be identified with the Hilbert space of the system, the holonomy of $A$ is to be identified with the time-evolution operator around $C$, and the trace over $R$ is the usual trace over the Hilbert space that defines the partition function in the Hamiltonian formalism.

Because the notion of tracing over a Hilbert space is inherently quantum mechanical, any attempts to perform essentially classical path integral manipulations involving the expressions in (4.4) or (4.6) are misguided at best. Rather, if we hope to generalize the simple, semi-classical path integral manipulations of Section 3 to apply to the Wilson loop path integral in (4.2), we need to use an alternative description for the Wilson loop operator that is itself semi-classical.

\footnotetext{
${ }^{8}$ In principle, a term linear in $(A-\kappa \Phi)$ also appears in the series expansion, but this term vanishes identically when $G$ is simply-connected and simple, as we assume.

${ }^{9}$ Though we will not make use of the following observation in this paper, the path integral over $\Phi$ in (4.3) can be performed directly, just as in Section 3, in the very special case that $C$ is a Legendrian curve [49] in $M$. By definition, if $C$ is a Legendrian curve, the tangent vector to $C$ lies everywhere in the kernel of $\kappa$. In this situation, $\Phi$ completely decouples from the generalized Wilson loop operator $\mathcal{W}_{R}(C)$ in (4.4), and the ordinary Wilson loop operator $W_{R}(C)$ is automatically shift-invariant. However, we do not wish to make any special assumptions about $C$ at the moment. For one reason, if $M$ is a Seifert manifold with the $U(1)$ invariant contact structure introduced in Section 3.2, the pullback of $\kappa$ to each Seifert fiber is non-vanishing by construction, so the Seifert fibers are not Legendrian.
} 
"ATMP-17-1-A1-BEA" — 2013/5/15 - 19:32 — page 35 — \#35

More precisely, we want to replace the quantum mechanical trace over $R$ in (4.6) by a path integral over an auxiliary bosonic field $U$ which is attached to the curve $C$ and coupled to the connection $A$ as a background field, so that schematically

$$
W_{R}(C)=\int \mathcal{D} U \exp \left[\operatorname{ics}\left(U ;\left.A\right|_{C}\right)\right]
$$

Here $\operatorname{cs}_{\alpha}\left(U ;\left.A\right|_{C}\right)$ is an action, depending upon the representation $R$ through its highest weight $\alpha$, which is a local, gauge-invariant functional of the auxiliary field $U$ and the restriction of $A$ to $C$. Not surprisingly, this semi-classical description (4.7) of $W_{R}(C)$ turns out to be the key ingredient required to reformulate the Wilson loop path integral in a shift-invariant fashion.

The idea of representing the Wilson loop operator by a path integral as in (4.7) is far from new. In the context of Chern-Simons theory, this device has already been applied to the canonical quantization of the theory by Elitzur and collaborators in [47]. As will be clear, though, the path integral description (4.7) of the Wilson loop operator holds much more generally for any gauge theory in any dimension. In the context of four-dimensional Yang-Mills theory, the semi-classical description of $W_{R}(C)$ is then much older, going back (at least) to work of Balachandran et al. [16] in the 1970s. See $[4,39,60]$ for other appearances of this idea, including a recent application to the volume conjecture for the colored Jones polynomial in [41].

Despite such history, we now review in some detail how the path integral description (4.7) of the Wilson loop operator works. We do so both for sake of completeness and to introduce a few geometric ideas which become necessary later. See also Section 7.7 of [37] for a very clear and somewhat more concise exposition of the following material.

As we have indicated, the basic idea behind the path integral description (4.7) of $W_{R}(C)$ is very simple. We interpret the closed curve $C$ as a periodic "time" for the field $U$, and we apply the Hamiltonian formalism to rewrite the path integral over $U$ axiomatically as the quantum mechanical trace of the corresponding time-evolution operator around $C$,

$$
W_{R}(C)=\operatorname{Tr}_{\mathcal{H}} P \exp \left(-\mathrm{i} \oint_{C} \mathbf{H}\right) .
$$

Here $\mathcal{H}$ is the Hilbert space, which we obtain by quantizing the field $U$; and $\mathbf{H}$ is the Hamiltonian, which acts upon $\mathcal{H}$ to generate infinitesimal translations along $C$. 
Comparing the conventional description of the Wilson loop operator in (4.6) to the axiomatic expression in (4.8), we see that the two agree if we identify ${ }^{10}$

$$
\begin{aligned}
R & \longleftrightarrow \mathcal{H} \\
P \exp \left(-\oint_{C} A\right) & \longleftrightarrow P \exp \left(-\mathrm{i} \oint_{C} \mathbf{H}\right) .
\end{aligned}
$$

Hence to make the Wilson loop path integral in (4.7) precise, we need only exhibit a classical theory on $C$, for which the gauge group $G$ acts as a symmetry, such that upon quantization we obtain a Hilbert space $\mathcal{H}$ isomorphic to $R$ and for which the time-evolution operator around $C$ is given by the holonomy of $A$, acting as an element of $G$ on $R$.

\subsubsection{Coadjoint orbits of $G$}

Now, of the two identifications in (4.9), the more fundamental by far is the identification of the irreducible representation $R$ with a Hilbert space, obtained by quantizing some classical phase space upon which $G$ acts as a symmetry. So before we even consider what classical theory must live on $C$ to describe the Wilson loop operator, we can ask the simpler and more basic question - what classical phase space must we quantize to obtain $R$ as a Hilbert space?

This question is beautifully answered by the Borel-Weil-Bott theorem [24], which explains how to obtain each irreducible representation of $G$ by quantizing a corresponding coadjoint orbit. Though the quantum interpretation of the Borel-Weil-Bott theorem is quite standard (see for instance Section 15 of [78]), we non-etheless review it now. Once we do so, we will find it very easy to exhibit the classical defect theory that must live on $C$ to describe the Wilson loop operator.

More or less as a means to establish notation and conventions, let us first recall some elementary facts about the geometry of the coadjoint orbits of $G$. We first fix a maximal torus $T \subset G$, for which $\mathfrak{t} \subset \mathfrak{g}$ is the associated Cartan subalgebra. By definition, the coadjoint orbits of $G$ are embedded in the dual $\mathfrak{g}^{*}$ of the Lie algebra $\mathfrak{g}$. However, given the invariant metric on

\footnotetext{
${ }^{10}$ We follow the standard physical definition according to which $\mathbf{H}$ is a Hermitian operator, accounting for the ' $-i$ ' in (4.8). We also recall that the gauge field $A$ is valued in the Lie algebra $\mathfrak{g}$, so $A$ is anti-Hermitian and no " $i$ " appears in the holonomy.
} 
$\mathfrak{g}$ defined by the pairing

$$
(x, y)=-\operatorname{Tr}(x y), \quad x, y \in \mathfrak{g},
$$

we are free to identify $\mathfrak{g} \cong \mathfrak{g}^{*}$ and hence equivalently to consider the adjoint orbits of $G$ embedded in $\mathfrak{g}$ itself. Though perhaps slightly unnatural from a purely mathematical perspective, we find the latter convention convenient. Thus, given an element $\lambda \in \mathfrak{t}$, we let $\mathcal{O}_{\lambda} \subset \mathfrak{g}$ be the orbit through $\lambda$ under the adjoint action of $G$.

Alternatively, $\mathcal{O}_{\lambda}$ can be regarded as a quotient $G / G_{\lambda}$, where $G_{\lambda}$ is the subgroup of $G$ which fixes $\lambda$ under the adjoint action. If $\lambda$ is a generic element of $\mathfrak{t}$, then $G_{\lambda}=T$, in which case $\lambda$ is said to be regular. However, $G_{\lambda}$ can be strictly larger than $T$, culminating in the extreme case that $\lambda=0$ and $G_{\lambda}=G$. The distinction between $\lambda$ regular and irregular will at times be important, but for the moment we treat these cases uniformly.

As our notation suggests, we assume that $G_{\lambda}$ acts on $G$ from the right, so that the quotient $G / G_{\lambda}$ is defined by the relation

$$
g \sim g h^{-1}, \quad g \in G, \quad h \in G_{\lambda} .
$$

With this convention, we identify $G / G_{\lambda}$ with $\mathcal{O}_{\lambda}$ via the map

$$
g G_{\lambda} \longmapsto g \lambda g^{-1},
$$

and under (4.12), the left-action of $G$ on itself descends to a transitive action of $G$ on $\mathcal{O}_{\lambda}$.

In order to discuss the quantization of $\mathcal{O}_{\lambda}$ as a classical phase space, we must endow $\mathcal{O}_{\lambda}$ with additional geometric structure, starting with a coadjoint symplectic form $\nu_{\lambda}$. To describe the coadjoint symplectic form explicitly, we introduce the canonical left-invariant one-form $\theta$ on $G$ which is valued in $\mathfrak{g}$,

$$
\theta=g^{-1} d g, \quad g \in G
$$

Using the form ' $\mathrm{Tr}$ ', we then define a real-valued, pre-symplectic one-form $\Theta_{\lambda}$ on $G$,

$$
\Theta_{\lambda}=-(\lambda, \theta)=\operatorname{Tr}(\lambda \theta),
$$

in terms of which we set

$$
\nu_{\lambda}=d \Theta_{\lambda}=\frac{1}{2}(\lambda,[\theta, \theta])=-\frac{1}{2}(\theta,[\lambda, \theta]) .
$$

In passing from (4.14) to (4.15), we use that $d \theta=-\theta \wedge \theta=-\frac{1}{2}[\theta, \theta]$. 
Quite literally, we have written $\nu_{\lambda}$ in (4.15) as a two-form on $G$. However, since $G_{\lambda}$ preserves $\lambda$ under the adjoint action, $\nu_{\lambda}$ is invariant under both the left-action of $G$ and the right-action of $G_{\lambda}$ and vanishes upon contraction with any vector tangent to $G_{\lambda}$, so this two-form descends to an invariant two-form on $\mathcal{O}_{\lambda}$.

As a two-form on $\mathcal{O}_{\lambda}$, clearly $\nu_{\lambda}$ is closed. Furthermore, if we let $\mathfrak{g}_{\lambda}$ denote the Lie algebra of the stabilizer group $G_{\lambda}$ and $\mathfrak{g} \ominus \mathfrak{g}_{\lambda}$ denote the orthocomplement to $\mathfrak{g}_{\lambda}$ in $\mathfrak{g}$, then the adjoint action of $\lambda$ on $\mathfrak{g} \ominus \mathfrak{g}_{\lambda}$ is by definition non-degenerate, with non-zero eigenvalues. Identifying $\mathfrak{g} \ominus \mathfrak{g}_{\lambda}$ geometrically with the tangent space to $\mathcal{O}_{\lambda}=G / G_{\lambda}$ at the identity coset, we see from (4.15) that $\nu_{\lambda}$ is thus non-degenerate as a symplectic form on $\mathcal{O}_{\lambda}$.

\subsubsection{The coadjoint moment map}

One goal of the present discussion is to keep careful track of the various signs and orientations which eventually enter our localization computations. We have already chosen a particular sign in the definition (4.14) of the pre-symplectic one-form $\Theta_{\lambda}$, and hence in the definition of the invariant symplectic form $\nu_{\lambda}$. This choice of sign has a particularly felicitous consequence for the moment map which describes the infinitesimal action of $G$ on $\mathcal{O}_{\lambda}$.

To compute the moment map for the action of $G$ on $\mathcal{O}_{\lambda}$, we identify $\mathcal{O}_{\lambda}$ with the quotient $G / G_{\lambda}$ under the map in (4.12). The vector field $V(\phi)$ on $\mathcal{O}_{\lambda}$ generated by an element $\phi \in \mathfrak{g}$ is then given at the coset $g G_{\lambda}$ simply by

$$
\delta g=\phi \cdot g \text {. }
$$

From (4.15), we also see that

$$
\iota_{V(\phi)} \nu_{\lambda}=\iota_{V(\phi)} d \Theta_{\lambda}=-d\left(\iota_{V(\phi)} \Theta_{\lambda}\right) .
$$

In passing to the second equality in (4.17), we use that the Lie derivative $£_{V(\phi)}=\left\{d, \iota_{V(\phi)}\right\}$ along $V(\phi)$ annihilates the invariant one-form $\Theta_{\lambda}$. Thus via (2.3) and (4.14), the moment map $\mu: \mathcal{O}_{\lambda} \rightarrow \mathfrak{g}^{*}$ for the action of $G$ on $\mathcal{O}_{\lambda}$ is given by

$$
\langle\mu, \phi\rangle=-\iota_{V(\phi)} \Theta_{\lambda}=\left(g \lambda g^{-1}, \phi\right)=-\operatorname{Tr}\left[\left(g \lambda g^{-1}\right) \cdot \phi\right] .
$$

An arbitrary constant could a priori appear in (4.18), but only if we set this constant to zero does the moment map $\mu$ satisfy the Hamiltonian 
"ATMP-17-1-A1-BEA" — 2013/5/15 - 19:32 — page 39 — \#39

condition in (2.4). Hence, $G$ acts in a Hamiltonian fashion on $\mathcal{O}_{\lambda}$, with moment map given by the natural embedding $\mathcal{O}_{\lambda} \subset \mathfrak{g} \cong \mathfrak{g}^{*}$ in (4.12).

\subsection{3 $\mathcal{O}_{\lambda}$ as a Kähler manifold}

In a nutshell, the Borel-Weil-Bott theorem concerns the algebraic geometry of the orbit $\mathcal{O}_{\lambda}$. So, we must also introduce a complex structure $\mathcal{J}$ on $\mathcal{O}_{\lambda}$. Like the coadjoint symplectic form, $\mathcal{J}$ will be invariant under $G$. Additionally, $\mathcal{J}$ will be compatible with $\nu_{\lambda}$ in the sense that $\nu_{\lambda}(\cdot, \mathcal{J} \cdot)$ defines a homogeneous Kähler metric on $\mathcal{O}_{\lambda}$ for which $\nu_{\lambda}$ is the Kähler form. The existence of such a complex structure can be understood in various ways. Here we take a rather down-to-earth approach and exhibit $\mathcal{J}$ directly as an invariant tensor on $\mathcal{O}_{\lambda}$.

At the outset, we find it convenient to introduce the complexified Lie algebras $\mathfrak{t}_{\mathbb{C}}=\mathfrak{t} \otimes \mathbb{C}, \mathfrak{g}_{\mathbb{C}}=\mathfrak{g} \otimes \mathbb{C}$, and $\mathfrak{g}_{\lambda, \mathbb{C}}=\mathfrak{g}_{\lambda} \otimes \mathbb{C}$. The invariant metric (4.10) on $\mathfrak{g}$ extends immediately to a Hermitian metric on $\mathfrak{g}_{\mathbb{C}}$, and we identify the Hermitian complement $\mathfrak{g}_{\mathbb{C}} \ominus \mathfrak{g}_{\lambda, \mathbb{C}}$ with the complexified tangent space to $\mathcal{O}_{\lambda}$ at the identity coset.

Any invariant tensor on $\mathcal{O}_{\lambda}$ is determined by its value at a single point. Hence, an invariant complex structure $\mathcal{J}$ on $\mathcal{O}_{\lambda}$ can be described algebraically as a splitting of the complexified tangent space at the identity coset into two complementary, half-dimensional subspaces $\mathfrak{g}^{(1,0)}$ and $\mathfrak{g}^{(0,1)}$,

$$
\mathfrak{g}_{\mathbb{C}} \ominus \mathfrak{g}_{\lambda, \mathbb{C}}=\mathfrak{g}^{(1,0)} \oplus \mathfrak{g}^{(0,1)},
$$

which we declare to consist of the respective holomorphic and antiholomorphic tangent vectors at that point and upon which $\mathcal{J}$ acts with eigenvalues $\pm i$.

The splitting in (4.19) can be obtained from any decomposition of the root system $\mathfrak{R}$ of $G$ into positive and negative subsets $\mathfrak{R}_{ \pm}$, so that $\mathfrak{R}=\mathfrak{R}_{+} \cup \mathfrak{R}_{-}$. As standard, we often write $\beta>0$ for positive roots $\beta \in \mathfrak{R}_{+}$, and similarly $\beta<0$ for negative roots $\beta \in \mathfrak{R}_{-}$.

Of course, the decomposition $\mathfrak{R}=\mathfrak{R}_{+} \cup \mathfrak{R}_{-}$is not unique, and different choices are related by the action of the Weyl group $\mathfrak{W}$ of $G$. However, in the generic case that $\lambda \in \mathfrak{t}$ is regular, we can fix this ambiguity by requiring that $\lambda$ itself lies in the positive Weyl chamber $C_{+} \subset \mathfrak{t}$. We recall that $C_{+}$is the polyhedral cone, with vertex at the origin, defined by the inequalities

$$
\mathrm{C}_{+}=\{\xi \in \mathfrak{t} \mid\langle\beta, \xi\rangle \geq 0 \text { for all } \beta>0\} .
$$


Thus, if $\lambda$ is regular, we simply take $\mathfrak{R}_{+}$to be the subset of $\beta \in \mathfrak{R}$ such that $\langle\beta, \lambda\rangle>0$. Again, we often write $\lambda \geq 0$ to indicate that $\lambda$ lies in $\mathrm{C}_{+}$, with strict positivity when $\lambda$ is regular.

If $\lambda$ is not regular, then inevitably some non-zero roots $\beta_{\perp} \in \mathfrak{R}$ satisfy $\left\langle\beta_{\perp}, \lambda\right\rangle=0$. Such $\beta_{\perp} \in \mathfrak{R}$ can be identified as roots of the stabilizer group $G_{\lambda}$. If $G_{\lambda}$ is non-trivial, we simply pick $\mathfrak{R}_{ \pm}$so that $\lambda$ lies on a boundary wall of the positive Weyl chamber $C_{+}$. This choice is determined up to the action of the Weyl group $\mathfrak{W}_{\lambda}$ of $G_{\lambda}$. By definition, $\lambda$ is fixed by $\mathfrak{W}_{\lambda}$, and as will be clear, the ambiguity under $\mathfrak{W}_{\lambda}$ is an unbroken gauge symmetry, which eventually factors out of our computations.

Given the decomposition $\mathfrak{R}=\mathfrak{R}_{+} \cup \mathfrak{R}_{-}$, we obtain an associated decomposition of $\mathfrak{g}_{\mathbb{C}} \ominus \mathfrak{t}_{\mathbb{C}}$ into positive and negative rootspaces $\mathfrak{g}_{ \pm}$, so that $\mathfrak{g}_{\mathbb{C}} \ominus \mathfrak{t}_{\mathbb{C}}=$ $\mathfrak{g}_{+} \oplus \mathfrak{g}_{-}$. Briefly, to define $\mathfrak{g}_{ \pm}$themselves, we recall that the rootspace $\mathfrak{e}_{\beta}$ associated to any non-zero root $\beta$ is the one-dimensional eigenspace in $\mathfrak{g}_{\mathbb{C}} \ominus \mathfrak{t}_{\mathbb{C}}$ upon which elements $\xi \in \mathfrak{t}$ act via the Lie bracket with eigenvalue $+\mathrm{i}\langle\beta, \xi\rangle$. That is, if $x_{\beta}$ is an element of $\mathfrak{e}_{\beta}$,

$$
\left[\xi, x_{\beta}\right]=+\mathrm{i}\langle\beta, \xi\rangle x_{\beta}, \quad x_{\beta} \in \mathfrak{e}_{\beta}
$$

The rootspaces $\mathfrak{g}_{ \pm}$are then given by the direct sums of the eigenspaces $\mathfrak{e}_{\beta}$ for positive and negative $\beta$,

$$
\mathfrak{g}_{+}=\bigoplus_{\beta \in \Re_{+}} \mathfrak{e}_{\beta}, \quad \mathfrak{g}_{-}=\bigoplus_{\beta \in \Re_{-}} \mathfrak{e}_{\beta}
$$

We now meet a crucial sign. To define the holomorphic and antiholomorphic tangent spaces $\mathfrak{g}^{(1,0)}$ and $\mathfrak{g}^{(0,1)}$ in $(4.19)$, we naturally use the positive and negative rootspaces $\mathfrak{g}_{ \pm}$. But which of $\mathfrak{g}_{+}$and $\mathfrak{g}_{-}$is to be holomorphic?

The answer to this question is determined by the requirement that $\nu_{\lambda}$ $(\cdot, \mathcal{J} \cdot)$ defines a positive-definite, as opposed to negative-definite, Hermitian form on the tangent space to $\mathcal{O}_{\lambda}$. Using the definition of $\nu_{\lambda}$ in (4.15) and the convention that $\lambda \geq 0$, one can check that the correct assignment is

$$
\mathfrak{g}^{(1,0)}=\left(\mathfrak{g}_{\mathbb{C}} \ominus \mathfrak{g}_{\lambda, \mathbb{C}}\right) \cap \mathfrak{g}_{+}, \quad \mathfrak{g}^{(0,1)}=\left(\mathfrak{g}_{\mathbb{C}} \ominus \mathfrak{g}_{\lambda, \mathbb{C}}\right) \cap \mathfrak{g}_{-} .
$$

In taking the intersection of $\mathfrak{g}_{\mathbb{C}} \ominus \mathfrak{g}_{\lambda, \mathbb{C}}$ with $\mathfrak{g}_{+}$to define the holomorphic tangent space $\mathfrak{g}^{(1,0)}$, we allow for the possibility that $\lambda$ is irregular. If $\lambda$ is 
"ATMP-17-1-A1-BEA" — 2013/5/15 - 19:32 — page 41 — \#41

regular and $\mathfrak{g}_{\lambda}=\mathfrak{t}$, then the assignment in (4.23) merely reduces to ${ }^{11}$

$$
\mathfrak{g}^{(1,0)}=\mathfrak{g}_{+}, \quad \mathfrak{g}^{(0,1)}=\mathfrak{g}_{-}, \quad \lambda>0 \quad \text { regular. }
$$

With $\mathfrak{g}^{(1,0)}$ and $\mathfrak{g}^{(0,1)}$ in hand, we immediately define the tensor $\mathcal{J}$ as an invariant almost-complex structure on $\mathcal{O}_{\lambda}$. By virtue of the elementary Lie algebra relation $\left[\mathfrak{g}_{+}, \mathfrak{g}_{+}\right] \subseteq \mathfrak{g}_{+}$, the Nijenhuis tensor associated to this almost-complex structure vanishes, so that $\mathcal{J}$ in fact defines an honest, integrable complex structure on $\mathcal{O}_{\lambda}$. Finally, because the Hermitian metric on $\mathfrak{g}_{\mathbb{C}}$ only pairs elements of $\mathfrak{g}_{+}$with elements of $\mathfrak{g}_{-}$, and because $\lambda$ preserves these spaces under the adjoint action, we see directly from (4.15) that $\nu_{\lambda}$ has holomorphic/anti-holomorphic type $(1,1)$ with respect to $\mathcal{J}$. Thus $\nu_{\lambda}$ and $\mathcal{J}$ together endow the orbit $\mathcal{O}_{\lambda}$ with a homogeneous Kähler structure.

\subsubsection{A prequantum line bundle on $\mathcal{O}_{\lambda}$}

From a physical perspective, the Borel-Weil-Bott theorem explains how to quantize $\mathcal{O}_{\lambda}$ as a Kähler manifold, so that is what we shall now do. We follow the recipe of geometric quantization, for which a nice reference is [109]. Admittedly, the following exposition puts the cart before the horse, since the notions of geometric quantization were developed partly based upon this example.

In general, quantization is a delicate procedure. Athough $\lambda$ parametrizes a continuous family of Kähler orbits in $\mathfrak{g}$, only a discrete subset of this family can actually be quantized. Specifically, according to the usual BohrSommerfeld condition, the symplectic form $\nu_{\lambda}$ must derive from a corresponding prequantum line bundle over $\mathcal{O}_{\lambda}$. By definition, a prequantum line bundle on any symplectic manifold $X$ with symplectic form $\Omega$ is a unitary line bundle with connection whose curvature is given by $+i \Omega$. In the present setting, as we now explain, the coadjoint symplectic form $\nu_{\lambda}$ on $\mathcal{O}_{\lambda}$ derives from a prequantum line bundle precisely when $\lambda \in \mathfrak{t}$ is quantized as a weight of $G$.

The last statement may require a minor clarification. The weight lattice $\Gamma_{\mathrm{wt}} \subset \mathfrak{t}^{*}$ of $G$ is defined intrinsically as a lattice in the dual $\mathfrak{t}^{*}$ of the Cartan subalgebra. However, we once again use the invariant metric (4.10) on $\mathfrak{g}$ to

\footnotetext{
${ }^{11}$ At this stage, a warning is in order. In more algebraic approaches to the BorelWeil-Bott theorem, for which $G / T=G_{\mathbb{C}} / B$ is presented as the quotient of the associated complex group $G_{\mathbb{C}}$ by a Borel subgroup $B$, the standard convention is to take the roots of $B$ to be positive roots of $G$. Hence in much of the literature, the holomorphic tangent space $\mathfrak{g}^{(1,0)}$ is identified with the negative rootspace $\mathfrak{g}_{-}$, opposite to (4.23). That convention ultimately leads to a characterization of $R$ via lowest (as opposed to highest) weights, which we prefer to avoid.
} 
identify $\mathfrak{t} \cong \mathfrak{t}^{*}$, under which the dual $\lambda^{*} \in \mathfrak{t}^{*}$ of $\lambda$ is defined via the relation $\left\langle\lambda^{*}, \cdot\right\rangle=(\lambda, \cdot)=-\operatorname{Tr}(\lambda \cdot)$. So by the quantization of $\lambda$ as a weight of $G$, we mean literally that $\lambda^{*}$ is quantized as an element of $\Gamma_{\mathrm{wt}}$.

Henceforth, to avoid cluttering the notation, we implicitly apply the isomorphism $\mathfrak{t} \cong \mathfrak{t}^{*}$ induced by '-Tr' and do not attempt to distinguish $\lambda$ from $\lambda^{*}$. By the same token, if $\alpha \in \Gamma_{\mathrm{wt}}$ is a weight of $G$, we do not distinguish $\alpha$ from its dual in $\mathfrak{t}$.

To explain why $\lambda$ must be quantized as a weight of $G$, we classify the possible line bundles on the coadjoint orbits of $G$. As an immediate simplifying observation, all coadjoint orbits $\mathcal{O}_{\lambda}$ take the form of quotients $G / G_{\lambda}$, where the stabilizer $G_{\lambda}$ necessarily contains the torus $T$. Under the quotient map from $G / T$ to $G / G_{\lambda}$, any line bundle on $G / G_{\lambda}$ immediately pulls back to a line bundle on $G / T$. So we need only classify line bundles on $G / T$.

As usual, line bundles on $G / T$ are classified topologically by elements of $H^{2}(G / T ; \mathbb{Z})$. On the other hand, $G / T$ sits tautologically as the base of a principal $T$-bundle whose total space is $G$,

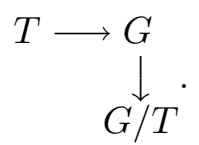

The Leray spectral sequence ${ }^{12}$ associated to the fibration in (4.25) implies that

$$
H^{2}(G / T ; \mathbb{Z})=H^{1}(T ; \mathbb{Z})=\operatorname{Hom}(T, U(1))
$$

For our application to Chern-Simons theory, we assume that the group $G$ is simply connected. The lattice of homomorphisms from $T$ to $U(1)$ is then isomorphic to the weight lattice of $G$,

$$
\Gamma_{\mathrm{wt}} \cong \operatorname{Hom}(T, U(1)) .
$$

Thus, at the level of topology, line bundles on $G / T$ are in one-to-one correspondence with weights of $G$.

The relation between line bundles on $G / T$ and weights of $G$ can be understood more concretely as follows. For each weight $\alpha \in \Gamma_{\mathrm{wt}}$, we let $\varrho_{\alpha}$ be the

\footnotetext{
${ }^{12}$ For an excellent introduction to spectral sequences, see Section 14 of [25].
} 
"ATMP-17-1-A1-BEA" — 2013/5/15 — 19:32 — page 43 — \#43

corresponding homomorphism,

$$
\varrho_{\alpha}: T \longrightarrow U(1) .
$$

Explicitly, $\varrho_{\alpha}$ is given in terms of $\alpha$ by

$$
\varrho_{\alpha}(t)=\exp [\mathrm{i}\langle\alpha, \xi\rangle], \quad t=\exp (\xi), \quad \xi \in \mathfrak{t} .
$$

As indicated in (4.29), $\xi$ is a logarithm of $t \in T$. This logarithm is only defined up to shifts $\xi \mapsto \xi+2 \pi y$, where $y \in \mathfrak{t}$ satisfies the integrality condition

$$
\exp (2 \pi y)=1 .
$$

Equivalently, each element $y$ in (4.30) corresponds to a homomorphism from $U(1)$ to $T$. By definition, the lattice of such homomorphisms is the cocharacter lattice of $G$,

$$
\Gamma_{\text {cochar }} \cong \operatorname{Hom}(U(1), T),
$$

which for simply-connected $G$ reduces to the coroot lattice $\Gamma_{\text {cort }}$. In any event, because the lattices in (4.27) and (4.31) are canonically dual over $\mathbb{Z}$, the weight $\alpha$ satisfies $\langle\alpha, y\rangle \in \mathbb{Z}$ for all $y \in \Gamma_{\text {cochar }}$, and the homomorphism $\varrho_{\alpha}$ is well defined.

Using the homomorphism in (4.29), we now introduce the associated line bundle $\mathfrak{L}(\alpha)$ over $G / T$,

$$
\mathfrak{L}(\alpha)=G \times \varrho_{\varrho_{\alpha}} \mathbb{C} .
$$

Here " $\varnothing_{\varrho_{\alpha}}$ " indicates that elements in the product $G \times \mathbb{C}$ are identified under the action of $T$ as

$$
t \cdot(g, v)=\left(g t^{-1}, \varrho_{\alpha}(t) \cdot v\right), \quad g \in G, \quad v \in \mathbb{C}, \quad t \in T .
$$

Thus, each weight $\alpha$ determines a complex line bundle $\mathfrak{L}(\alpha)$. Conversely, since $H^{2}(G ; \mathbb{Z})=0$ under our assumptions on $G$, any line bundle on $G / T$ pulls back to a topologically-trivial line bundle on $G$ and hence can be represented by a quotient of $G \times \mathbb{C}$ as in (4.33). Finally, if $\alpha$ happens to be an irregular weight, the homomorphism $\varrho_{\alpha}$ in (4.29) extends uniquely to a homorphism from $G_{\alpha}$ to $U(1)$, and $\mathfrak{L}(\alpha)$ is the pullback from a corresponding line bundle on $G / G_{\alpha}$.

To interpret $\mathfrak{L}(\alpha)$ as a prequantum line bundle, we still need to endow $\mathfrak{L}(\alpha)$ with a Hermitian metric and a compatible unitary connection, both of 
which are invariant under $G$. Thankfully, the metric and the connection are straightforward to describe.

For the metric, we note that sections of $\mathfrak{L}(\alpha)$ can be identified with complex functions $f$ on $G$ which transform equivariantly under the action of $T$,

$$
f\left(g t^{-1}\right)=\varrho_{\alpha}(t) \cdot f(g), \quad g \in G, \quad t \in T .
$$

Hence the invariant Hermitian metric for complex functions on $G$, unique up to norm, immediately descends to an invariant Hermitian metric for sections of $\mathfrak{L}(\alpha)$.

Since the metric on $\mathfrak{L}(\alpha)$ is invariant under $G$, a compatible unitary connection on $\mathfrak{L}(\alpha)$ must be invariant as well. This observation, along with our explicit description of $\varrho_{\alpha}$, suffices to fix the connection uniquely.

Of course, one can describe a connection on a line bundle in many ways. As we have already defined $\mathfrak{L}(\alpha)$ in terms of the principal $T$-bundle in (4.25), we also describe its connection in these terms. Globally, we take the invariant unitary connection on $\mathfrak{L}(\alpha)$ to be a left-invariant one-form $B$ on $G$, which is valued in the Lie algebra of $U(1)$, identified with $+\mathrm{i} \mathbb{R}$, and for which $d_{B}=d+B$ is the covariant derivative acting on sections of $\mathfrak{L}(\alpha)$, identified as in (4.34) with equivariant functions on $G$. Covariance of $d_{B}$ then implies that $d_{B} \varrho_{\alpha}(t)=0$, so $B$ must be given in terms of the left-invariant Cartan form $\theta$ by

$$
B=-\mathrm{i}\langle\alpha, \theta\rangle=\mathrm{i} \Theta_{\alpha} .
$$

Since $\Theta_{\alpha}$ is the pre-symplectic one-form satisfying $\nu_{\alpha}=d \Theta_{\alpha}$, the coadjoint symplectic form $\nu_{\lambda}$ on $\mathcal{O}_{\lambda}$ derives from a prequantum line bundle precisely when $\lambda=\alpha$ is quantized as a weight of $G$, in which case the prequantum line bundle is $\mathfrak{L}(\alpha)$. Actually, because $\lambda$ lies by convention in the positive Weyl chamber, $\alpha \geq 0$ is necessarily a highest weight for $G$.

\subsubsection{The Borel-Weil-Bott theorem in brief}

At this point, the statement of the Borel-Weil-Bott theorem is remarkably simple. ${ }^{13}$ Because $\nu_{\alpha}$ is the Kähler form for an invariant Kähler metric on $\mathcal{O}_{\alpha}$, the prequantum line bundle $\mathfrak{L}(\alpha)$ immediately becomes a holomorphic line bundle of positive curvature over $\mathcal{O}_{\alpha}$. As such, the prequantum line bundle naturally admits global holomorphic sections, and via the usual

\footnotetext{
${ }^{13}$ For sake of time, we regrettably omit the refinements due to Bott [24].
} 
"ATMP-17-1-A1-BEA" — 2013/5/15 - 19:32 — page $45-\# 45$

recipe of geometric quantization, we construct a Hilbert space $\mathcal{H}$ for $\mathcal{O}_{\alpha}$ as the space of holomorphic sections of $\mathfrak{L}(\alpha)$. That is,

$$
\mathcal{H}=H \frac{0}{\partial}\left(\mathcal{O}_{\alpha}, \mathfrak{L}(\alpha)\right), \quad \alpha \geq 0 .
$$

On the other hand, the holomorphic sections of $\mathfrak{L}(\alpha)$ automatically transform in a finite-dimensional representation of $G$ induced from its action on $\mathcal{O}_{\alpha}$. Perhaps not surprisingly, the role of the Borel-Weil-Bott theorem is to identify this representation as none other than the irreducible representation $R$ with highest weight $\alpha$,

$$
R \cong H \frac{0}{\partial}\left(\mathcal{O}_{\alpha}, \mathfrak{L}(\alpha)\right) .
$$

In these terms, the Borel-Weil-Bott isomorphism (4.37) exhibits $R$ as a Hilbert space obtained by quantizing $\mathcal{O}_{\alpha}$ as a classical phase space.

Though we have introduced the general adjoint orbit $\mathcal{O}_{\alpha}$, the Borel-WeilBott theorem can be (and often is) stated purely in terms of the maximal orbit described by $G / T$. As mentioned earlier, if $\alpha$ is not a regular weight of $G$, the prequantum line bundle $\mathfrak{L}(\alpha)$ over $\mathcal{O}_{\alpha}=G / G_{\alpha}$ pulls back to a holomorphic line bundle over $G / T$. The holomorphic sections of the pullback also transform on $G / T$ in the representation $R$, so at least from the perspective of algebraic geometry, the irreducible representations of $G$ can be constructed more uniformly using holomorphic line bundles on $G / T$ alone.

However, if $\alpha$ is not a regular weight, the closed two-form $\nu_{\alpha}$ is degenerate on $G / T$. As an extreme example, if $\alpha=0$, then $\mathfrak{L}(\alpha)$ is the trivial line bundle, and $\nu_{\alpha}=0$. Of course, in that case $\mathcal{O}_{\alpha}$ is simply a point. So to interpret $\mathfrak{L}(\alpha)$ as a prequantum line bundle associated to a necessarily nondegenerate symplectic form, we prefer to state the Borel-Weil-Bott theorem with $\mathfrak{L}(\alpha)$ being a line bundle over the general adjoint orbit $\mathcal{O}_{\alpha}$, as opposed to the maximal orbit given by $G / T$.

\subsubsection{An elementary example}

Although we have given a careful description of the prequantum line bundle $\mathfrak{L}(\alpha)$, it would unfortunately take us a bit too far afield to demonstrate here the crucial Borel-Weil-Bott isomorphism in (4.37). However, see for instance Section 23 of [52], particularly Exercise 23.62, for a nice proof.

In place of a proof, we end our geometric digression with an elementary example of the Borel-Weil-Bott theorem in action. Namely, we let $G$ be $S U(2)$, so that $T$ is $U(1)$, and we identify $G / T$ with $S^{2}$ via the Hopf fibration. To endow $G / T$ with a complex structure, we pick a positive Weyl chamber 
of $S U(2)$, corresponding to an orientation for $S^{2}$, after which we regard $S^{2}$ as $\mathbb{C P}^{1}$.

To illustrate our conventions explicitly, we parameterize elements $g$ of $S U(2)$ by means of complex variables $(X, Y) \in \mathbb{C}^{2}$, so that

$$
g=\left(\begin{array}{cc}
\bar{Y} & X \\
-\bar{X} & Y
\end{array}\right), \quad|X|^{2}+|Y|^{2}=1 .
$$

Similarly, we parameterize elements $t$ in the maximal torus $T$ by means of an angular variable $\theta$,

$$
t=\left(\begin{array}{cc}
\mathrm{e}^{\mathrm{i} \theta} & 0 \\
0 & \mathrm{e}^{-\mathrm{i} \theta}
\end{array}\right), \quad \theta \in[0,2 \pi] .
$$

Under the action $g \mapsto g \cdot t^{-1}$, the variables $(X, Y)$ then transform homogeneously with unit charge, $(X, Y) \mapsto\left(\mathrm{e}^{i \theta} X, \mathrm{e}^{i \theta} Y\right)$. As for the complex structure on $G / T$, we pick the positive Weyl chamber so that the positive rootspace $\mathfrak{g}_{+}$of $S U(2)$ is spanned by strictly upper-triangular matrices,

$$
\mathfrak{g}_{+}=\mathbb{C} \cdot\left(\begin{array}{ll}
0 & 1 \\
0 & 0
\end{array}\right) .
$$

Conversely, $\mathfrak{g}_{-}$is spanned by lower triangular matrices. With this convention, $[X: Y]$ become homogeneous coordinates on $\mathbb{C P}^{1}$.

The weights of $S U(2)$ are labelled by a single integer $m$. If we use the invariant metric ' $-\operatorname{Tr}$ ' to dualize $\mathfrak{t} \cong \mathfrak{t}^{*}$, the weights take the concrete form

$$
\alpha=\frac{m}{2}\left(\begin{array}{cc}
i & 0 \\
0 & -i
\end{array}\right), \quad m \in \mathbb{Z},
$$

such that $\alpha$ is positive when $m \geq 0$. The associated homomorphism $\varrho_{\alpha}$ : $T \rightarrow U(1)$ in (4.29) then becomes $\varrho_{\alpha}(t)=\exp (i m \theta)$, and the holomorphic line bundle $\mathfrak{L}(\alpha)$ defined in (4.32) and (4.33) is precisely the line bundle of degree, or monopole number, $m$ on $\mathbb{C P}^{1}$.

Via (4.34), we see that holomorphic sections of $\mathfrak{L}(\alpha)$ are given by degree $m$ polynomials in the homogeneous coordinates $[X: Y]$ of $\mathbb{C P}^{1}$. Under the action of $S U(2)$, these polynomials transform naturally in the irreducible representation of dimension $m+1$, realizing the Borel-Weil-Bott isomorphism in (4.37). Finally, the Kähler metric on $\mathbb{C P}^{1}$ compatible with the curvature of the invariant connection on $\mathfrak{L}(\alpha)$ for $m>0$ is just a multiple of the round, Fubini-Study metric. 
"ATMP-17-1-A1-BEA" — 2013/5/15 - 19:32 — page 47 — \#47

\subsubsection{More about quantum mechanics on coadjoint orbits}

Our discussion of the Borel-Weil-Bott theorem so far has been fairly abstract. As the next step towards explaining the path integral description of $W_{R}(C)$, let us place the isomorphism in (4.37) into its proper physical context.

To start, we clearly want to consider the quantum mechanics of a single particle moving on the orbit $\mathcal{O}_{\alpha}$. Formally, such a particle is described by a one-dimensional sigma model of maps $U$ from $S^{1}$ to $\mathcal{O}_{\alpha}$,

$$
U: S^{1} \longrightarrow \mathcal{O}_{\alpha}
$$

Although not particularly essential at the moment, we take the particle worldline to be compact in anticipation of our application to the Wilson loop operator, where the abstract $S^{1}$ will be identified with the embedded curve $C \subset M$.

We now want to pick a classical action for $U$ such that the orbit $\mathcal{O}_{\alpha}$, with symplectic form $\nu_{\alpha}$, appears as the corresponding classical phase space. Once we do so, we can immediately invoke the Borel-Weil-Bott isomorphism (4.37) to identify the Hilbert space $\mathcal{H}$ obtained by quantizing $U$ with the representation $R$. But what classical action for $U$ should we pick?

An immediate guess might be to consider the standard two-derivative sigma model action associated to the invariant Kähler metric on $\mathcal{O}_{\alpha}$ defined by $\nu_{\alpha}$ and $\mathcal{J}$,

$$
S_{\sigma}(U)=\frac{1}{2} \oint_{S^{1}} \eta^{-\frac{1}{2}} \nu_{\alpha}(d U, \mathcal{J} \cdot d U)=\frac{1}{2} \oint_{S^{1}} \eta^{-\frac{1}{2}}\left(\gamma_{\alpha}\right)_{m n} \frac{d U^{m}}{d \tau} \frac{d U^{n}}{d \tau} .
$$

Here, $\tau$ is a coordinate along $S^{1}$, and $\eta \equiv \eta_{\tau \tau}$ is a worldline metric on $S^{1}$. Also, $\gamma_{\alpha}=\nu_{\alpha}(\cdot, \partial \cdot)$ is the invariant Kähler metric on $\mathcal{O}_{\alpha}$. Finally, for sake of concreteness, we parameterize the map $U$ using local coordinates $u^{m}$ on $\mathcal{O}_{\alpha}$, in terms of which we write the second expression in (4.43).

Though $S_{\sigma}(U)$ is a natural action to consider, it cannot be correct for two reasons. First and foremost, $S_{\sigma}(U)$ describes a particle freely moving on $\mathcal{O}_{\alpha}$, and the classical phase space for this particle is not the orbit $\mathcal{O}_{\alpha}$ but its cotangent bundle $T^{*} \mathcal{O}_{\alpha}$, with the standard cotangent symplectic structure. The Hilbert space obtained by quantizing $T^{*} \mathcal{O}_{\alpha}$ would then be the infinite-dimensional space $L^{2}\left(\mathcal{O}_{\alpha}\right)$ of square-integrable functions on $\mathcal{O}_{\alpha}$, as opposed to the finite-dimensional representation $R$. Second, the sigma model action $S_{\sigma}(U)$ depends upon the choice of a worldline metric $\eta$ on $S^{1}$. 
However, the Wilson loop operator $W_{R}(C)$ certainly does not depend upon the choice of a metric on the corresponding curve $C$.

As these objections suggest, the correct action for $U$ should be of firstorder, not second-order, in the "time" derivative $d / d \tau$ along $S^{1}$, so that the classical phase space has a chance to be compact. Furthermore, the action for $U$ should be topological in the sense that it does not depend upon the choice of a metric on $S^{1}$.

The requirements above are hallmarks of a Chern-Simons action. Given that we already possess an invariant unitary connection $\Theta_{\alpha}$ on the line bundle $\mathfrak{L}(\alpha)$, let us consider the following Chern-Simons-type action for $U$,

$$
\operatorname{cs}_{\alpha}(U)=\oint_{S^{1}} U^{*}\left(\Theta_{\alpha}\right)=\oint_{S^{1}}\left(\Theta_{\alpha}\right)_{m} \frac{d U^{m}}{d \tau}
$$

Here $U^{*}\left(\Theta_{\alpha}\right)$ denotes the pullback of $\Theta_{\alpha}$ to a connection over $S^{1}$, and $\operatorname{cs}_{\alpha}(U)$ is quite literally the one-dimensional Chern-Simons action for $U^{*}\left(\Theta_{\alpha}\right)$. Once again, in the second expression of (4.44) we write the pullback $U^{*}\left(\Theta_{\alpha}\right)$ using coordinates $u^{m}$ on $\mathcal{O}_{\alpha}$, in terms of which $\Theta_{\alpha}$ is represented locally by the one-form $\left(\Theta_{\alpha}\right)_{m} d u^{m}$.

As with any Chern-Simons action, the functional $\operatorname{cs}_{\alpha}(U)$ is not strictly invariant under "large", homotopically non-trivial gauge transformations on $S^{1}$. However, so long as $\alpha \in \Gamma_{\mathrm{wt}}$ is quantized as a weight of $G$ and hence $+i \Theta_{\alpha}$ is defined as an honest $U(1)$-connection over $\mathcal{O}_{\alpha}$, the value of $\operatorname{cs}_{\alpha}(U)$ is well-defined modulo $2 \pi$, a sufficient condition to discuss a sensible path integral.

From a more physical perspective, the first-order action for $U$ in (4.44) specifies the minimal coupling of a charged particle on $\mathcal{O}_{\alpha}$ to a background magnetic field given by the coadjoint symplectic form $\nu_{\alpha}=d \Theta_{\alpha}$. From this perspective, the quantization of $\alpha$ as a weight of $G$ follows from the quantization of flux on a compact space. The dynamics of such a charged particle moving on $\mathcal{O}_{\alpha}$ in the background magnetic field $\nu_{\alpha}$ are then described by the total action

$$
S_{\text {tot }}(U)=S_{\sigma}(U)+\operatorname{cs}_{\alpha}(U) .
$$

To pass from (4.45) to (4.44) alone, we consider the low-energy limit, for which $\eta \rightarrow \infty$. In this limit, the two-derivative sigma model action $S_{\sigma}(U)$ in (4.45) becomes irrelevant, and the topological Chern-Simons-type term 
"ATMP-17-1-A1-BEA" — 2013/5/15 - 19:32 — page 49 — \#49

$\operatorname{cs}_{\alpha}(U)$ provides an effective action for the Landau groundstates of the particle. ${ }^{14}$

To proceed with the analysis of the topological sigma model with action $\operatorname{cs}_{\alpha}(U)$, we first determine the classical phase space for $U$. Under a variation $\delta U$, the variation of the first-order action $\operatorname{cs}_{\alpha}(U)$ is given by

$$
\delta \operatorname{cs}_{\alpha}(U)=\oint_{S^{1}} \nu_{\alpha}(\delta U, d U)=\oint_{S^{1}}\left(\nu_{\alpha}\right)_{m n} \delta U^{m} \frac{d U^{n}}{d \tau} .
$$

Once again, the first equality in (4.46) derives from the relation $\nu_{\alpha}=d \Theta_{\alpha}$.

Since $\nu_{\alpha}$ is non-degenerate as a symplectic form on $\mathcal{O}_{\alpha}$, the equations of motion which follow from (4.46) imply that $d U^{n} / d \tau=0$. Thus, classical solutions for $U$ are constant maps, and the classical phase space for $U$ is the orbit $\mathcal{O}_{\alpha}$ itself, as we originally required. Furthermore, if we consider $\delta U^{n}$ to represent a linearized coordinate on a neighborhood of the identity coset in $\mathcal{O}_{\alpha}$, then we see from (4.46) that the canonical momentum conjugate to $\delta U^{n}$ is $\Pi_{n}=\left(\nu_{\alpha}\right)_{m n} \delta U^{m}$. Hence the classical Poisson bracket on $\mathcal{O}_{\alpha}$ derives from the coadjoint symplectic form $\nu_{\alpha}$.

To quantize the compact phase space for $U$, we immediately invoke our previous, more abstract discussion of the Borel-Weil-Bott theorem. Namely, because of the background magnetic field $\nu_{\alpha}$, the wavefunctions for $U$ in the Kähler polarization of $\mathcal{O}_{\alpha}$ transform as holomorphic sections of the prequantum line bundle $\mathfrak{L}(\alpha)$, where by convention $\alpha \geq 0$. Thus, the Hilbert space for $U$ is again $\mathcal{H}=H \frac{0}{\partial}\left(\mathcal{O}_{\alpha}, \mathfrak{L}(\alpha)\right)$, and in the more physical language above, $\mathcal{H}$ can be interpreted as the space of Landau levels of a single electron moving on $\mathcal{O}_{\alpha}$ in the magnetic field $\nu_{\alpha}$. The Borel-Weil-Bott theorem (4.37) then asserts that these Landau levels transform under $G$ in the representation $R$.

\subsubsection{Coupling to the bulk gauge field}

The one-dimensional Chern-Simons sigma model with target space $\mathcal{O}_{\alpha}$ realizes the first identification in (4.9) required to describe the Wilson loop operator $W_{R}(C)$ semi-classically. To realize the second identification in (4.9), we simply attach the sigma model to the curve $C \subset M$, and we couple the

\footnotetext{
${ }^{14}$ Even in the limit $\eta \rightarrow \infty$, the classical action $\operatorname{cs}_{\alpha}(U)$ may receive a non-trivial oneloop correction when we perform the functional integral over non-zero Fourier modes of $U$ in the sigma model with action $S_{\text {tot }}(U)$. Such a quantum correction can at most shift the weight $\alpha$ to a new weight $\alpha^{\prime}$ and thus does not alter our general analysis of the effective topological sigma model with action $\operatorname{cs}_{\alpha}(U)$. In Section 7, we compute explicitly a related quantum shift in $\alpha$.
} 
sigma model field $U$ to the bulk connection $A$ by promoting the global action of $G$ on $\mathcal{O}_{\alpha}$ to a gauge symmetry.

The action of $G$ on $\mathcal{O}_{\alpha}$ is perhaps most transparent when the orbit is described as a quotient $G / G_{\alpha}$. Even before we gauge the Chern-Simons sigma model, let us apply this description of $\mathcal{O}_{\alpha}$ to rewrite the functional $\operatorname{cs}_{\alpha}(U)$ in (4.44) a bit more concretely. Given the sigma model map $U$ with target $\mathcal{O}_{\alpha}=G / G_{\alpha}$, we lift $U$ to a map

$$
g: S^{1} \longrightarrow G
$$

Here $U$ and $g$ are related via $U=g \alpha g^{-1}$, just as in (4.12). In terms of $g$, we then rewrite $\operatorname{cs}_{\alpha}(U)$ using the explicit description (4.14) of $\Theta_{\alpha}$ as a left-invariant one-form on $G$,

$$
\operatorname{cs}_{\alpha}(U)=\oint_{S^{1}} U^{*}\left(\Theta_{\alpha}\right)=\oint_{S^{1}} \operatorname{Tr}\left(\alpha \cdot g^{-1} d g\right) .
$$

The expression for $\operatorname{cs}_{\alpha}(U)$ in (4.48) is admirably explicit, but let us quickly consider how it depends upon the choice of $g$. First, a lift of $U$ to $g$ always exists over $S^{1}$. The obstruction to lifting $U$ is measured by a characteristic class of degree two on $\mathcal{O}_{\alpha}$, which vanishes for trivial reasons when pulled back to $S^{1}$ under $U$. However, of course $g$ is only determined by $U$ up to the local right-action of $G_{\alpha}$, under which a map $h: S^{1} \rightarrow G_{\alpha}$ acts on $g$ as $g \mapsto g h^{-1}$. If $h$ is homotopically trivial, one can easily check that value of $\operatorname{cs}_{\alpha}(U)$ in (4.48) is invariant under this transformation. Otherwise, under "large" gauge transformations by homotopically non-trivial maps $h: S^{1} \rightarrow G_{\alpha}$, the value of the functional in (4.48) generally shifts by an integral multiple of $2 \pi$, reflecting the harmless ambiguity in a ChernSimons action.

In order to forestall potential confusion, let me emphasize that the lift from $U$ to $g$ in (4.48) serves merely as a convenient device to describe the functional $\operatorname{cs}_{\alpha}(U)$ in a manifestly $G$-invariant fashion. All path integrals that we discuss will be integrals over the space of sigma model maps $U: S^{1} \rightarrow \mathcal{O}_{\alpha}$, as opposed to the space of maps $g: S^{1} \rightarrow G$.

We now attach the sigma model with target space $\mathcal{O}_{\alpha}$ to the curve $C \subset M$, and we gauge the global action of $G$ on $\mathcal{O}_{\alpha}$. The procedure of gauging the sigma model on $C$ is entirely straightforward. Nevertheless, at some risk of pedantry, we provide a systematic discussion.

In order to keep track of the local action of $G$ in the sigma model, we recall that the bulk gauge theory on $M$ is associated to a fixed principal 
"ATMP-17-1-A1-BEA" — 2013/5/15 — 19:32 — page 51 — \#51

$G$-bundle $P$ over $M$ on which $A$ is a connection,

$$
G \longrightarrow \underset{\substack{\downarrow \\ M}}{P}
$$

The restriction of $P$ to the embedded curve $C \subset M$ determines a principal $G$-bundle $\left.P\right|_{C}$ over $C$, and the map $g$ in (4.47) now transforms as a section of $\left.P\right|_{C}$. Finally, the sigma model field $U$ itself transforms geometrically as a section of an associated bundle $Q$ with fiber $\mathcal{O}_{\alpha}$ over $C$,<smiles>[CH]O[O]</smiles>

Explicitly, using the same notation as in (4.32), $Q$ is given by $\left.P\right|_{C} \times_{G} \mathcal{O}_{\alpha}$.

As a homogeneous bundle on $\mathcal{O}_{\alpha}$, the prequantum line bundle $\mathfrak{L}(\alpha)$ extends fiberwise in (4.50) to a line bundle over $Q$. This line bundle carries its own unitary connection, determined by both $\Theta_{\alpha}$ and the bulk connection $A$ to be

$$
\Theta_{\alpha}(A)=\operatorname{Tr}\left(\alpha \cdot g^{-1} d_{A} g\right),
$$

where

$$
d_{A} g=d g+\left.A\right|_{C} \cdot g .
$$

As one can readily check, the covariant derivative in (4.52) behaves correctly under a gauge transformation acting on $A$ and $g$ as

$$
\delta A=-d_{A} \phi, \quad \delta g=\left.\phi\right|_{C} \cdot g,
$$

where $\phi$ is a section of the adjoint bundle $\operatorname{ad}(P)$ on $M$. Hence the expression for $\Theta_{\alpha}(A)$ in (4.51) is invariant under gauge transformations in $G$ on $M$ and otherwise transforms like $\Theta_{\alpha}$ as a unitary connection over $Q$.

Using $\Theta_{\alpha}(A)$, we now write the gauge-invariant version of the sigma model action on $C$ as

$$
\begin{aligned}
\operatorname{cs}_{\alpha}\left(U ;\left.A\right|_{C}\right) & =\oint_{C} U^{*}\left(\Theta_{\alpha}(A)\right)=\oint_{C}\left(\Theta_{\alpha}\right)_{m} \frac{d_{A} U^{m}}{d \tau}, \\
& =\oint_{C} \operatorname{Tr}\left(\alpha \cdot g^{-1} d_{A} g\right) .
\end{aligned}
$$


For concreteness, in the first line of (4.54) we again write the sigma model action in terms of local coordinates $u^{m}$ on $\mathcal{O}_{\alpha}$, and we have introduced there the covariant derivative $d_{A}$ acting on $U$. The action of $d_{A}$ on $U$ is inherited immediately from its action on $g$ in (4.52).

\subsubsection{A semi-classical description of the Wilson loop operator}

The gauged sigma model on $C$ with target space $\mathcal{O}_{\alpha}$ finally provides the promised semi-classical description for the Wilson loop operator. In short,

$$
\begin{aligned}
& W_{R}(C)=\epsilon^{\Delta_{\alpha} / 2} \int_{L \mathcal{O}_{\alpha}} \mathcal{D} U \exp \left[\operatorname{ics}\left(U ;\left.A\right|_{C}\right)\right], \\
& \epsilon=\frac{2 \pi}{k}, \quad \Delta_{\alpha}=\operatorname{dim} L \mathcal{O}_{\alpha} .
\end{aligned}
$$

Formally, the sigma model path integral in (4.55) is an integral over the space of sections of the bundle $Q$ in (4.50). If we are willing to forget that this space carries an action by the group $\mathcal{G}$ of gauge transformations and thence to trivialize $Q$, the space of sections of $Q$ can be equivalently regarded as the free loopspace $L \mathcal{O}_{\alpha}$ of the orbit $\mathcal{O}_{\alpha}$. We find the latter notation convenient in (4.55) and will phrase the discussion in terms of $L \mathcal{O}_{\alpha}$ throughout the remainder of the paper.

As is standard for sigma models, the loopspace $L \mathcal{O}_{\alpha}$ carries an invariant metric induced from the invariant Kähler metric on $\mathcal{O}_{\alpha}$, and the path integral measure $\mathcal{D} U$ is the associated Riemannian measure on $L \mathcal{O}_{\alpha}$.

For our particular application to Chern-Simons theory, we have also multiplied the path integral in (4.55) by a formal power of the Chern-Simons coupling $\epsilon$. Because the relevant power is half the dimension of $L \mathcal{O}_{\alpha}$, this prefactor can be equivalently absorbed into the Riemannian measure $\mathcal{D} U$ on $L \mathcal{O}_{\alpha}$ if the sigma model metric on $L \mathcal{O}_{\alpha}$ is rescaled by $\epsilon$. Indeed, this rescaling is the ultimate reason we introduce the prefactor at all. As will prove convenient, we write $\epsilon L \mathcal{O}_{\alpha}$ to indicate the loopspace $L \mathcal{O}_{\alpha}$ equipped with the sigma model metric induced from the invariant Kähler metric on $\epsilon \mathcal{O}_{\alpha} \equiv \mathcal{O}_{\epsilon \alpha}$. In that abbreviated notation,

$$
W_{R}(C)=\int_{\epsilon L \mathcal{O}_{\alpha}} \mathcal{D} U \exp \left[i \operatorname{cs}_{\alpha}\left(U ;\left.A\right|_{C}\right)\right]
$$

with no errant prefactor. We will explain the need for the effective rescaling $\mathcal{O}_{\alpha} \mapsto \epsilon \mathcal{O}_{\alpha}$ later, in Section 4.3. 
"ATMP-17-1-A1-BEA" — 2013/5/15 — 19:32 — page 53 - \#53

We are left to establish the relationship between the semi-classical description of the Wilson loop operator in (4.56) and its conventional description as the trace in $R$ of the holonomy of $A$ around $C$. As we indicated initially, this task amounts to demonstrating the correspondence

$$
\begin{aligned}
\mathcal{H} & \longleftrightarrow R \\
P \exp \left(-i \oint_{C} \mathbf{H}\right) & \longleftrightarrow P \exp \left(-\oint_{C} A\right) .
\end{aligned}
$$

We have already discussed how the quantization of $U$ leads to the first identification in (4.57) before we couple to $A$, and the corresponding story in the gauged sigma model proceeds essentially as before. From the gaugeinvariant action in (4.54), we immediately deduce that $U$ satisfies the equation of motion $d_{A} U^{n}=0$ and hence must be covariantly constant. ${ }^{15}$ As a result, all classical trajectories for $U$ are determined uniquely by parallel transport from an arbitrary initial value $U_{0}$.

Very explicitly, if we pass from the curve $C$ with a chosen basepoint to the universal cover $\mathbb{R}$, then the classical time-evolution of $U$ is given by

$$
U(\tau)=P \exp \left(-\int_{0}^{\tau} A\right) \cdot U_{0}, \quad U_{0} \in \mathcal{O}_{\alpha}
$$

Here $\tau$ is a time-coordinate along $\mathbb{R}$, with $0 \in \mathbb{R}$ being a lift of the basepoint on $C$. The minus sign in (4.58) arises from our convention that $d_{A}=d+A$. We now identify the classical phase space for $U$ with the coadjoint orbit $\mathcal{O}_{\alpha}$, as parameterized by the initial value $U_{0}$ in (4.58). Similarly, the Poisson bracket on the phase space is still determined by the coadjoint symplectic form $\nu_{\alpha}$, and the Hilbert space $\mathcal{H}$ for $U$ is again isomorphic to $R$.

To establish the second identification in (4.57), we must consider the time-evolution in the gauged sigma model. As (4.58) indicates, the classical time-evolution in this theory is not completely trivial, insofar as it depends upon parallel transport using the restriction of the connection $A$ to $C$. Hence the classical time-evolution around $C$ is given by the holonomy of $A$, acting as an element of $G$ on the phase space $\mathcal{O}_{\alpha}$.

Since we identify the Hilbert space for $U$ with the space of holomorphic sections of the prequantum line bundle $\mathfrak{L}(\alpha)$, the action of the classical timeevolution operator on $\mathcal{O}_{\alpha}$ immediately lifts to a corresponding quantum action on $\mathcal{H} \cong R$. Thus as we require in (4.57), the quantum time-evolution

\footnotetext{
${ }^{15}$ Equivalently, the lift $g$ satisfies $\left[\alpha, g^{-1} d_{A} g\right]=0$ and is covariantly constant up to the right-action of $G_{\alpha}$.
} 
operator in the gauged sigma model is given by the holonomy of $A$ around $C$, acting as an element of $G$ on $R$.

The following remark is not essential, but it may resolve a small puzzle for some readers. To pass from the cover $\mathbb{R}$ to $C$ in the discussion above, we make the periodic identification $\tau \sim \tau+1$. For $U(\tau)$ in (4.58) to be single-valued under this identification, the holonomy $P \exp \left(-\oint_{C} A\right)$ must preserve the initial value $U_{0} \in \mathcal{O}_{\alpha}$ under the adjoint action of $G$. As a result, the classical holonomy of $A$ around $C$ is not arbitrary but lies in the centralizer of $U_{0}$, a subgroup of $G$ conjugate to the stabilizer $G_{\alpha}$.

At first glance, this restriction on $P \exp \left(-\oint_{C} A\right)$ might seem to conflict with the usual definition of the Wilson loop operator, for which the holonomy around $C$ is arbitrary. However, the stabilizer group $G_{\alpha}$ always contains the maximal torus $T$, so as $U_{0}$ ranges over points in $\mathcal{O}_{\alpha}$, the centralizer of $U_{0}$ ranges over all of $G$. Once we integrate over $U_{0}$ in the semi-classical description (4.56) of $W_{R}(C)$, the holonomy of $A$ around $C$ is therefore unrestricted. For this reason, the appearance in (4.56) of the free, as opposed to based, loopspace $L \mathcal{O}_{\alpha}$ is crucial.

\subsection{A new formulation of Chern-Simons theory, part II}

In obtaining the identifications in (4.57), we treat the gauge field $A$ as a fixed background connection on $M$. As a result, the semi-classical description of the Wilson loop operator in (4.56) is completely general, applicable to any gauge theory in any dimension. This elegant little idea seems not to have found significant application in four-dimensional Yang-Mills theory, which may be one reason that the idea has remained somewhat obscure. Nonetheless, as a small bit of cosmic justice, the semi-classical description of $W_{R}(C)$ proves to be tailor-made for our study of Wilson loop operators in Chern-Simons theory.

We return to the basic Wilson loop path integral in (4.2), which we now formulate using (4.56) as a path integral over the product $\mathcal{A} \times \epsilon L \mathcal{O}_{\alpha}$,

$$
\begin{aligned}
Z(\epsilon ; C, R)= & \frac{1}{\operatorname{Vol}(\mathcal{G})}\left(\frac{1}{2 \pi \epsilon}\right)^{\Delta_{\mathcal{G}}} \int_{\mathcal{A} \times \epsilon L \mathcal{O}_{\alpha}} \mathcal{D} A \mathcal{D} U \\
& \times \exp \left[\frac{\mathrm{i}}{2 \epsilon} \operatorname{cs}(A)+\mathrm{i} \operatorname{cs}_{\alpha}\left(U ;\left.A\right|_{C}\right)\right] .
\end{aligned}
$$

Our primary goal in the remainder of this section is to cast the Wilson loop path integral (4.59) into a shift-invariant form, just as we did for 
"ATMP-17-1-A1-BEA" — 2013/5/15 — 19:32 — page 55 — \#55

the Chern-Simons partition function in Section 3. Yet before we perform any path integral manipulations, let us quickly discuss the moduli space of classical solutions which arise as critical points of the joint action

$$
S_{0}(A, U)=\operatorname{CS}(A)+2 \epsilon \operatorname{cs}_{\alpha}\left(U ;\left.A\right|_{C}\right) .
$$

\subsubsection{On classical Wilson loops in Chern-Simons theory}

In order to discuss the equations of motion for $A$ and $U=g \alpha g^{-1}$, we first find it convenient to rewrite the topological sigma model action for $U$ in terms of a bulk integral over $M$,

$$
\operatorname{cs}_{\alpha}\left(U ;\left.A\right|_{C}\right)=\oint_{C} \operatorname{Tr}\left(\alpha \cdot g^{-1} d_{A} g\right)=\int_{M} \delta_{C} \wedge \operatorname{Tr}\left(\alpha \cdot g^{-1} d_{A} g\right) .
$$

In passing to the second expression in (4.61), we have introduced a two-form $\delta_{C}$ with delta-function support along $C$ to represent the Poincaré dual of this curve.

Varying $\operatorname{CS}(A)$ and $\operatorname{cs}_{\alpha}\left(U ;\left.A\right|_{C}\right)$ with respect to $A$ and $g$, we find that the classical equations of motion, which follow from the action in (4.60) are given by

$$
F_{A}+\epsilon\left(g \alpha g^{-1}\right) \cdot \delta_{C}=0, \quad\left[\alpha, g^{-1} d_{A} g\right]=0,
$$

or equivalently in terms of $U$,

$$
F_{A}+\epsilon U \cdot \delta_{C}=0, \quad d_{A} U=0 .
$$

The first equation in (4.63) implies that $A$ is a connection on $M$, which is flat away from $C$ and otherwise has delta-function curvature along $C$ fixed by the value of $U$ in $\mathcal{O}_{\alpha} \subset \mathfrak{g}$. Also, as we applied previously, the second equation in (4.63) asserts that $U$ is covariantly constant along $C$.

By way of notation, we let $\mathcal{N}(C, \alpha)$ denote the space of pairs $(A, U)$ solving the system in (4.63), modulo gauge transformations. In the special case $\alpha=0, \mathcal{M}(C, \alpha)$ immediately reduces to the moduli space $\mathcal{M}$ of flat connections on $M$, and as well-known, that moduli space admits a concrete, finite-dimensional presentation as the moduli space of homomorphisms $\varrho$ from the fundamental group $\pi_{1}(M)$ to the gauge group $G$,

$$
\mathcal{M}=\left\{\varrho: \pi_{1}(M) \rightarrow G\right\} / G .
$$

In terms of the gauge theory, $\varrho$ encodes the holonomies of a flat connection on $M$. 
As we review now, the extended moduli space $\mathcal{M}(C, \alpha)$ admits a completely analogous presentation, again as a moduli space of homomorphisms to $G$. Besides elucidating the classical interpretation of the Wilson loop operator, this global perspective on $\mathcal{M}(C, \alpha)$ proves to be a critical ingredient for our localization computations in Section 7.

To analyze $\mathcal{M}(C, \alpha)$, we first provide a local model for classical configurations of $(A, U)$ which satisfy the equations of motion (4.63) on a small tubular neighborhood $N_{C}$ of the curve $C$. Topologically $N_{C}$ is a solid torus, upon which we introduce cylindrical coordinates $(r, \varphi, \tau)$. Here $(r, \varphi)$ are polar coordinates on a plane transverse to $C$, which passes through the origin at $r=0$, and $\tau$ is an axial coordinate along $C$ with unit length. We assume that the standard orientation on $(r, \varphi, \tau)$ agrees with the given orientations on $M$ and $C$. Explicitly in terms of the cylindrical coordinates $(r, \varphi, \tau)$, the standard orientation on $N_{C}$ is given by the three-form $d r \wedge d \varphi \wedge d \tau$, and $C$ itself is oriented by $d \tau$.

Up to gauge transformations, local solutions to (4.63) can then be presented in terms of parameters $\left(U_{0}, V_{0}\right)$ as

$$
A=-\frac{\epsilon U_{0}}{2 \pi} d \varphi+V_{0} d \tau, \quad U(\tau)=U_{0} \in \mathcal{O}_{\alpha}
$$

Here $U_{0}$ is a constant taking values in the orbit $\mathcal{O}_{\alpha} \subset \mathfrak{g}$, and $V_{0}$ is any element of $\mathfrak{g}$ commuting with $U_{0}$, so that

$$
\left[U_{0}, V_{0}\right]=0 .
$$

Using the identity $d(d \varphi)=2 \pi \delta_{C}$, one can immediately check that the (4.65) solves the equations of motion in (4.63).

The description of $A$ in (4.65) holds on the neighborhood $N_{C}$. Away from $N_{C}$, classical configurations for $A$ are given by flat connections on the complement $M^{o}$,

$$
M^{o}=M-N_{C}
$$

Such a flat connection is determined up to gauge equivalence by its holonomies, which are now encoded by a homomorphism $\varrho^{o}$ from the fundamental group of the complement $M^{o}$ to $G$,

$$
\varrho^{o}: \pi_{1}\left(M^{o}\right) \longrightarrow G .
$$

In the special case that $M=S^{3}$, the group $\pi_{1}\left(M^{o}\right)$ is just the classical knot group of $C$. We will recall a few interesting examples of knot groups later, when we arrive at the computations in Section 7. 
Of course, $\varrho^{\circ}$ cannot be an arbitrary homomorphism in (4.68), since the holonomies of $A$ around $C$ and its meridian are already fixed in terms of $\left(U_{0}, V_{0}\right)$ in (4.65). As standard, by the meridian of $C$ we mean the distinguished element $m \in \pi_{1}\left(M^{o}\right)$, which is represented by the small circle about $C$ parameterized by $\varphi$ in (4.65) and oriented according to $d \varphi .^{16}$ Hence the homomorphism $\varrho^{\circ}$ in (4.68) must be related to the constant $U_{0}$ in (4.65) via

$$
\varrho^{o}(\mathrm{~m})=P \exp \left(-\oint_{\mathrm{m}} A\right)=\exp \left(\epsilon U_{0}\right), \quad \epsilon=\frac{2 \pi}{k}
$$

As $U_{0}$ varies in $\mathcal{O}_{\alpha}$, the holonomy around the meridian of $C$ therefore takes values in the conjugacy class $\mathfrak{C}_{\alpha / k} \subset G$ containing the group element $\exp (2 \pi \alpha / k)$,

$$
\varrho^{o}(\mathrm{~m}) \in \mathfrak{C}_{\alpha / k}=\operatorname{Cl}[\exp (2 \pi \alpha / k)]
$$

Here $\mathrm{Cl}[\cdot]$ indicates the conjugacy class in $G$ containing the given element. We alert the reader that we have included a convenient factor of $2 \pi$ in the definition of $\mathfrak{C}_{\alpha / k}$.

By the same token, the holonomy of $A$ around $C$ is fixed by $V_{0}$ in (4.65). Moreover, since $\left[U_{0}, V_{0}\right]=0$, the holonomy around $C$ necessarily commutes with the holonomy around $\mathrm{m}$, implying $\left[\varrho^{o}(C), \varrho^{o}(\mathrm{~m})\right]=1$. At first glance, one might worry that this condition must be imposed as an additional constraint on $\varrho^{o}(C)$, analogous to the constraint in (4.70). Thankfully, that worry is misplaced for the following elementary reason. Both $C$ and $\mathrm{m}$ are represented by curves on the boundary $\partial N_{C}$, a two-torus. Trivially, $C$ and $\mathrm{m}$ commute as elements of $\pi_{1}\left(\partial N_{C}\right)$, so they also commute as elements of $\pi_{1}(M)$. Hence, the images of $C$ and $\mathrm{m}$ under any homomorphism from $\pi_{1}(M)$ to $G$ automatically commute. As a result, the condition $\left[\varrho^{o}(C), \varrho^{o}(\mathrm{~m})\right]=1$ is not an independent constraint on $\varrho^{o}$, and we immediately eliminate the parameter $V_{0}$ in favor of the holonomy $\varrho^{\circ}(C)$ from the description of $\mathcal{M}(C, \alpha)$.

Combining the local and global descriptions of $A$ on $N_{C}$ and $M^{o}$ respectively, we present the extended moduli space $\mathcal{M}(C, \alpha)$ concretely as the space

\footnotetext{
${ }^{16}$ To characterize $\mathrm{m}$ more intrinsically, we note that the boundary $\partial N_{C}$ of $N_{C}$ is a two-torus. As a curve on that boundary, $m$ is then determined up to orientation as the generator of the kernel of the map from $\pi_{1}\left(\partial N_{C}\right) \cong \mathbb{Z} \times \mathbb{Z}$ to $\pi_{1}\left(N_{C}\right) \cong \mathbb{Z}$. To fix the orientation of $\mathrm{m}$, we require that the curve in $\partial N_{C}$ which is homotopic to $C$ itself to have positive intersection with $\mathrm{m}$, where $\partial N_{C}$ is oriented according to the outward orientation on $M^{o}=M-N_{C}$.
} 
of pairs $\left(\varrho^{o}, U_{0}\right)$ which satisfy the compatibility condition in (4.69), modulo the diagonal action of $G$. So as a formal quotient,

$$
\mathcal{M}(C, \alpha)=\left\{\left(\varrho^{o}, U_{0}\right) \mid \varrho^{o}(\mathrm{~m})=\exp \left(\epsilon U_{0}\right)\right\} / G \text {. }
$$

The description of $\mathcal{M}(C, \alpha)$ in (4.71) is still somewhat redundant. For generic $k \in \mathbb{Z}$ and $\alpha \in \Gamma_{\mathrm{wt}}$, the relation in (4.69) can be smoothly inverted to determine $U_{0}$ as a function of $\varrho^{o}(\mathrm{~m})$, thereby also eliminating $U_{0}$ from the description of $\mathcal{M}(C, \alpha)$. Hence $\mathcal{M}(C, \alpha)$ can be presented more succinctly as the moduli space of homomorphisms from $\pi_{1}\left(M^{o}\right)$ to $G$, which satisfy the necessary condition in (4.70),

$$
\begin{aligned}
\mathcal{M}(C, \alpha) & =\left\{\varrho^{o}: \pi_{1}\left(M^{o}\right) \rightarrow G \mid \varrho^{o}(\mathrm{~m}) \in \mathfrak{C}_{\alpha / k}\right\} / G, \\
\mathfrak{C}_{\alpha / k} & =\operatorname{Cl}[\exp (2 \pi \alpha / k)] .
\end{aligned}
$$

Although the technical conditions on $k$ and $\alpha$ under which $U_{0}$ can be eliminated from (4.71) are not so important now, they will be important later, and we state those conditions precisely in Section 7.1. (See also Section 5.2 for a warm-up discussion.)

To conclude our discussion of the classical Wilson loop, let us give two very simple examples of the extended moduli space $\mathcal{M}(C, \alpha)$. The first example will really be a non-example, meant only to illustrate that homomorphisms $\varrho^{o}: \pi_{1}\left(M^{o}\right) \rightarrow G$ satisfying the necessary condition in (4.70) may or may not exist, depending upon the structure of $\pi_{1}\left(M^{o}\right)$. So for a case in which $\mathcal{M}(C, \alpha)$ is actually empty, let $M$ be the product $S^{2} \times S^{1}$, and for any point in $S^{2}$, let $C$ be the corresponding $S^{1}$ fiber over that point. Then the meridian $\mathrm{m}$ of $C$ is contractible in $M^{o}$, and a homomorphism $\varrho^{o}$ satisfying (4.70) exists only if $\alpha=0$.

As a slightly more interesting example, one which we will considerably generalize in Section 7, let us consider the case that $M$ is $S^{3}$ and $C$ is the unknot. Then $\pi_{1}\left(M^{o}\right) \cong \mathbb{Z}$ is freely generated by $\mathrm{m}$, so the homomorphism $\varrho^{O}$ is determined once its value on $\mathrm{m}$ is fixed. Via $(4.69), \varrho^{O}(\mathrm{~m})$ is in turn determined by $U_{0} \in \mathcal{O}_{\alpha}$, and $\mathcal{M}(C, \alpha)$ is the quotient $\mathcal{O}_{\alpha} / G$. Since $G$ acts transitively on $\mathcal{O}_{\alpha}$, this quotient is just a point, with a non-trivial stabilizer $G_{\alpha}$.

\subsubsection{The shift-invariant Wilson loop operator}

We now arrive at our first main result, which is to extend the path integral manipulations in Section 3 to provide a shift-invariant reformulation of 
the Wilson loop path integral $Z(\epsilon ; C, R)$. Indeed, once we apply the semiclassical description of $W_{R}(C)$ to rewrite $Z(\epsilon ; C, R)$ as below,

$$
\begin{aligned}
Z(\epsilon ; C, R)= & \frac{1}{\operatorname{Vol}(\mathcal{G})}\left(\frac{1}{2 \pi \epsilon}\right)^{\Delta_{\mathcal{G}}} \int_{\mathcal{A} \times \epsilon L \mathcal{O}_{\alpha}} \mathcal{D} A \mathcal{D} U \\
& \times \exp \left[\frac{\mathrm{i}}{2 \epsilon} \operatorname{cs}(A)+i \operatorname{cs}_{\alpha}\left(U ;\left.A\right|_{C}\right)\right],
\end{aligned}
$$

the ideas in Section 3 extend in an almost embarrassingly straightforward fashion.

As in (4.3), we first consider the generalization of (4.73) obtained by replacing $A$ with the shift-invariant combination $A-\kappa \Phi$,

$$
Z(\epsilon ; C, R)=\frac{1}{\operatorname{Vol}(\mathcal{G})} \frac{1}{\operatorname{Vol}(\mathcal{S})}\left(\frac{1}{2 \pi \epsilon}\right)^{\Delta_{\mathcal{G}}} \int \mathcal{D} A \mathcal{D} U \mathcal{D} \Phi \exp \left[\frac{\mathrm{i}}{2 \epsilon} S(A, \Phi, U)\right]
$$

where

$$
S(A, \Phi, U)=\operatorname{CS}(A-\kappa \Phi)+2 \epsilon \operatorname{cs}_{\alpha}(U ; A-\kappa \Phi) .
$$

We assume that the shift symmetry $\mathcal{S}$ acts on $A$ and $\Phi$ just as before, and $\mathcal{S}$ acts trivially on $U$. Upon setting $\Phi=0$ with the shift symmetry, we reproduce (4.73) as before.

On the other hand, to underscore the significance of (4.74), let us expand the shift-invariant sigma model action $\operatorname{cs}_{\alpha}(U ; A-\kappa \Phi)$ in terms of $\Phi$. From (4.61), we immediately find

$$
\operatorname{cs}_{\alpha}(U ; A-\kappa \Phi)=\operatorname{cs}_{\alpha}(U ; A)-\int_{M} \kappa \wedge \delta_{C} \operatorname{Tr}\left[\left(g \alpha g^{-1}\right) \Phi\right] .
$$

The essential observation to make about (4.76) is simply that $\Phi$ appears linearly. Thus, $\Phi$ still enters the total shift-invariant action $S(A, \Phi, U)$ quadratically.

To be explicit, we expand $S(A, \Phi, U)$ in terms of $\Phi$ to obtain

$$
S(A, \Phi, U)=\operatorname{CS}(A)+2 \epsilon \operatorname{cs}_{\alpha}(U ; A)-\int_{M}\left[2 \kappa \wedge \operatorname{Tr}\left(\Phi \mathcal{F}_{A}\right)-\kappa \wedge d \kappa \operatorname{Tr}\left(\Phi^{2}\right)\right] .
$$

Here, as a convenient shorthand, we introduce a 'generalized' curvature $\mathcal{F}_{A}$ which includes the delta-function contribution from (4.76), 
so that

$$
\mathcal{F}_{A}=F_{A}+\epsilon\left(g \alpha g^{-1}\right) \delta_{C} .
$$

Since the integral over $\Phi$ in (4.74) is Gaussian, we perform it exactly as before. By virtue of the shift symmetry, the remaining integral over the affine space $\mathcal{A}$ then reduces to an integral over the quotient $\overline{\mathcal{A}}=\mathcal{A} / \mathcal{S}$, and we obtain the promised shift-invariant reformulation of the general Wilson loop path integral in Chern-Simons theory. Thus,

$$
Z(\epsilon ; C, R)=\frac{1}{\operatorname{Vol}(\mathcal{G})}\left(\frac{-\mathrm{i}}{2 \pi \epsilon}\right)^{\Delta_{\mathcal{G}} / 2} \int_{\overline{\mathcal{A}} \times \epsilon L \mathcal{O}_{\alpha}} \overline{\mathcal{D} A} \mathcal{D} U \exp \left[\frac{\mathrm{i}}{2 \epsilon} S(A, U)\right],
$$

where

$$
S(A, U)=\operatorname{CS}(A)+2 \epsilon \operatorname{cs}_{\alpha}\left(U ;\left.A\right|_{C}\right)-\int_{M} \frac{1}{\kappa \wedge d \kappa} \operatorname{Tr}\left[\left(\kappa \wedge \mathcal{F}_{A}\right)^{2}\right] .
$$

By construction, $S(A, U)$ is invariant under the shift $\delta A=\sigma \kappa$, where $\sigma$ is an arbitrary function on $M$ valued in the Lie algebra $\mathfrak{g}$ of the gauge group $G$. Alternatively, using the description of $\operatorname{cs}_{\alpha}\left(U ;\left.A\right|_{C}\right)$ in (4.61), one can verify the shift-invariance of $S(A, U)$ directly. Under the shift $\delta A=\sigma \kappa$, a new term linear in $\sigma$ arises from the defect action $\operatorname{cs}_{\alpha}\left(U ;\left.A\right|_{C}\right)$. This term is then canceled by the cross-term proportional to $\sigma$, which appears in the square of $\mathcal{F}_{A}$.

\subsubsection{A word about the Wilson link}

Although our discussion throughout will focus for simplicity on the case of a single Wilson loop operator, the previous path integral manipulations extend immediately to Wilson links in $M$.

To state the general result, we consider a product of Wilson loop operators associated to oriented curves $C_{\ell}$, which are linked in $M$ and decorated by irreducible representations $R_{\ell}$ with highest weights $\alpha_{\ell}$ for $\ell=1, \ldots$, L. On each curve, we introduce a corresponding sigma model field $U_{\ell}$, and we apply the semi-classical description of $W_{R}(C)$ in (4.56) to write the obvious generalization of (4.59),

$$
\begin{aligned}
& Z\left(\epsilon ;\left(C_{1}, R_{1}\right), \ldots,\left(C_{\mathrm{L}}, R_{\mathrm{L}}\right)\right)=\frac{1}{\operatorname{Vol}(\mathcal{G})}\left(\frac{1}{2 \pi \epsilon}\right)^{\Delta_{\mathcal{G}}} \\
& \quad \times \int_{\mathcal{A} \times \epsilon L \mathcal{O}_{\alpha_{1}} \times \cdots \times \epsilon L \mathcal{O}_{\alpha_{\mathrm{L}}}} \mathcal{D} A U_{1} \cdots \mathcal{D} U_{\mathrm{L}} \exp \left[\frac{\mathrm{i}}{2 \epsilon} \operatorname{cS}(A)+i \sum_{\ell=1}^{\mathrm{L}} \mathrm{cs}_{\alpha_{\ell}}\left(U_{\ell} ;\left.A\right|_{C}\right)\right] .
\end{aligned}
$$


"ATMP-17-1-A1-BEA" — 2013/5/15 — 19:32 — page 61 — \#61

Through the same manipulations as before, we find that the shift invariant version of the Wilson link path integral in (4.81) is given by

$$
\begin{aligned}
& Z\left(\epsilon ;\left(C_{1}, R_{1}\right), \ldots,\left(C_{\mathrm{L}}, R_{\mathrm{L}}\right)\right)=\frac{1}{\operatorname{Vol}(\mathcal{G})}\left(\frac{-\mathrm{i}}{2 \pi \epsilon}\right)^{\Delta_{\mathcal{G}} / 2} \\
& \quad \times \int_{\overline{\mathcal{A}} \times \epsilon L \mathcal{O}_{\alpha_{1}} \times \cdots \times \epsilon L \mathcal{O}_{\alpha_{\mathrm{L}}}} \overline{\mathcal{D} A} \mathcal{D} U_{1} \cdots \mathcal{D} U_{\mathrm{L}} \exp \left[\frac{i}{2 \epsilon} S\left(A, U_{1}, \cdots, U_{\mathrm{L}}\right)\right],
\end{aligned}
$$

where

$$
\begin{aligned}
S\left(A, U_{1}, \ldots, U_{\mathrm{L}}\right)= & \operatorname{cs}(A)+2 \epsilon \sum_{\ell=1}^{\mathrm{L}} \operatorname{cs}_{\alpha_{\ell}}\left(U_{\ell} ;\left.A\right|_{C}\right) \\
& -\int_{M} \frac{1}{\kappa \wedge d \kappa} \operatorname{Tr}\left[\left(\kappa \wedge \mathcal{F}_{A}\right)^{2}\right],
\end{aligned}
$$

with

$$
\mathcal{F}_{A}=F_{A}+\epsilon \sum_{\ell=1}^{\mathrm{L}}\left[\left(g \alpha g^{-1}\right) \delta_{C}\right]_{\ell} .
$$

In order to suppress the proliferation of subscripts in (4.84), the component index ' $\ell$ ' on the bracketed quantity applies to all elements therein.

Via (4.82), (4.83), and (4.84), we have succeeded in reformulating ChernSimons theory on an arbitrary contact three-manifold $M$ in such a way that one component of $A$ completely decouples from all Wilson loop observables. We now turn to a wonderful geometric consequence of this fact.

\subsection{The Seifert loop path integral as a symplectic integral}

Although the reformulation of the Wilson loop path integral in (4.79) is completely general, to interpret that path integral symplectically we must again specialize to the Seifert case.

As in Section 3.3, we assume $M$ to be a Seifert manifold, with a distinguished $U(1)_{\mathrm{R}}$ action and an invariant contact form $\kappa$. We also assume that the curve $C$ in (4.79) is a generic orbit of the $U(1)_{\mathrm{R}}$ action on $M$ and hence is the Seifert fiber over a smooth, non-orbifold point on the Riemann surface $\Sigma$ sitting at the base of $M$. With this assumption, we do not need to specify the particular point $p \in \Sigma$ over which $C$ sits, since the isotopy class of the 
embedding $C \subset M$ does not change under continuous variations of $p$. In the terminology of the Introduction section, $W_{R}(C)$ is then a Seifert loop operator in $M$. Later, in Section 7.1, we classify the possible Seifert loop operators when $M$ is $S^{3}$.

So far, an implicit question has been hanging over our quest to recast the Seifert loop path integral as a symplectic integral of the canonical form (1.3). As we reviewed in Section 3, the Chern-Simons partition function on a Seifert manifold is given by such an integral, determined by the Hamiltonian action of the group $\mathcal{H}=U(1)_{\mathrm{R}} \ltimes \widetilde{\mathcal{G}}_{0}$ on the symplectic space $\overline{\mathcal{A}}$. However, since the data of $\overline{\mathcal{A}}$ and $\mathcal{H}$ are intrinsically associated to Chern-Simons theory, and since the form of the canonical symplectic integral is, well, canonical, how can we hope to find yet another symplectic integral to describe the Seifert loop path integral?

As soon as we ask this question, a glance at (4.79) suffices to answer it. There we have written the general Wilson loop path integral as an integral over the product

$$
\overline{\mathcal{A}}_{\alpha}=\overline{\mathcal{A}} \times \epsilon L \mathcal{O}_{\alpha} .
$$

To summarize the chief miracle in this paper, when $M$ and $C$ are both Seifert, the space $\overline{\mathcal{A}}_{\alpha}$ is also a symplectic space upon which the Hamiltonian group $\mathcal{H}$ acts, and the Seifert loop path integral in (4.79) is the canonical symplectic integral associated to the data of $\overline{\mathcal{A}}_{\alpha}$ and $\mathcal{H}$.

\subsubsection{Hamiltonian symmetries of $L \mathcal{O}_{\alpha}$}

To explain the statement above, let us first consider the Hamiltonian action of $\mathcal{H}$ on $\overline{\mathcal{A}}_{\alpha}$. The group $\mathcal{H}$ will act on $\overline{\mathcal{A}}_{\alpha}$ in a diagonal fashion; since we have already discussed the Hamiltonian action of $\mathcal{H}$ on $\overline{\mathcal{A}}$, we need only discuss the Hamiltonian action of $\mathcal{H}$ on $L \mathcal{O}_{\alpha}$.

Briefly, $\mathcal{H}$ acts on $L \mathcal{O}_{\alpha}$ in the natural way. Gauge transformations on $M$ act on $L \mathcal{O}_{\alpha}$ by restriction to $C$, just as in (4.53). Because $C$ as a Seifert fiber of $M$ is preserved under rotations in $U(1)_{\mathrm{R}}$, the loopspace $L \mathcal{O}_{\alpha}$ also inherits the natural action by $U(1)_{R}$. Finally, the central $U(1)_{Z}$ in the extension $\widetilde{\mathcal{G}_{0}}$ acts trivially on $L \mathcal{O}_{\alpha}$, just as it does on $\overline{\mathcal{A}}$. Explicitly, if $(p, \phi, a)$ is a generator in the Lie algebra of $\mathcal{H}$ as in Section 3.3, the infinitesimal action of $(p, \phi, a)$ on $L \mathcal{O}_{\alpha}$ is given by

$$
\delta g=p £_{\mathrm{R}} g+\left.\phi\right|_{C} \cdot g .
$$

As in Section 4.2, $g$ is a section of the principal $G$-bundle $\left.P\right|_{C}$ over $C$ which we use to represent a point in $L \mathcal{O}_{\alpha}$. 
"ATMP-17-1-A1-BEA" — 2013/5/15 — 19:32 — page 63 - \#63

Besides a natural action by $\mathcal{H}$, the loopspace $L \mathcal{O}_{\alpha}$ also carries a natural symplectic form $\Upsilon_{\alpha}$, inherited from the canonical coadjoint symplectic form $\nu_{\alpha}$ on the orbit $\mathcal{O}_{\alpha}$ itself. As for any sigma model, the tangent space to $L \mathcal{O}_{\alpha}$ at the point corresponding to a given sigma model map $U: C \rightarrow \mathcal{O}_{\alpha}$ is the vector space of sections of the pullback by $U$ of the tangent bundle $T \mathcal{O}_{\alpha}$ to $\mathcal{O}_{\alpha}$. To present $\Upsilon_{\alpha}$ explicitly, we let $\eta$ and $\xi$ be sections of $U^{*}\left(T \mathcal{O}_{\alpha}\right)$ representing tangent vectors to $L \mathcal{O}_{\alpha}$. Then

$$
\Upsilon_{\alpha}(\eta, \xi)=\oint_{C} \kappa \nu_{\alpha}(\eta, \xi)
$$

We abuse notation slightly in (4.87), but hopefully the meaning of this expression is clear. The symplectic form $\nu_{\alpha}$ on $\mathcal{O}_{\alpha}$ induces a pointwise pairing on $C$ between the sections $\eta$ and $\xi$ of $U^{*}\left(T \mathcal{O}_{\alpha}\right)$, and we integrate the resulting function over $C$ using the contact form $\kappa$.

One can easily check that $\Upsilon_{\alpha}$ is a symplectic form on $L \mathcal{O}_{\alpha}$. For instance, because $\nu_{\alpha}$ is closed on $\mathcal{O}_{\alpha}$, the two-form $\Upsilon_{\alpha}$ is immediately closed on $L \mathcal{O}_{\alpha}$. Also, the non-degeneracy of $\Upsilon_{\alpha}$ follows from the non-degeneracy of $\nu_{\alpha}$, along with the observation that the pullback of $\kappa$ to $C$ is nowhere vanishing. ${ }^{17}$

Finally, since $\nu_{\alpha}$ is invariant under the action of $G$ on $\mathcal{O}_{\alpha}$ and since $\kappa$ is invariant under the action of $U(1)_{R}$ on $C$, the symplectic form $\Upsilon_{\alpha}$ is manifestly invariant under $\mathcal{H}$.

We are left to compute the moment map $\mu$, which describes the infinitesimal action (4.86) of $\mathcal{H}$ on $L \mathcal{O}_{\alpha}$. This moment map can actually be determined in two different ways, both of which are illuminating. To start, we take the direct approach.

From the expression for $\nu_{\alpha}$ in (4.15), we see that the contraction of $\Upsilon_{\alpha}$ with the vector field $V(p, \phi, a)$ on $L \mathcal{O}_{\alpha}$ generated by $(p, \phi, a)$ in (4.86) is given by

$$
\iota_{V(p, \phi, a)} \Upsilon_{\alpha}=\oint_{C} \kappa \operatorname{Tr}\left(\left(p g^{-1} £_{\mathrm{R}} g+g^{-1} \phi g\right) \cdot\left[\alpha, g^{-1} \delta g\right]\right)
$$

Here $g^{-1} \delta g$ is the left-invariant Cartan form on $L G$, where as usual, $\delta$ is best thought of as an infinite-dimensional version of the de Rham operator.

\footnotetext{
${ }^{17}$ Because $C$ is a Seifert fiber of $M$, the vector field $\mathrm{R}$, which generates the action of $U(1)_{R}$ is everywhere tangent to $C$. By its construction as an abelian connection on the Seifert fibration, $\kappa$ satisfies $\langle\kappa, R\rangle=1$ and hence provides a nowhere vanishing one-form on $C$.
} 
With a bit of calculation, the result in (4.88) can be rewritten as

$$
\iota_{V(p, \phi, a)} \Upsilon_{\alpha}=-\delta \oint_{C} \kappa \operatorname{Tr}\left[\alpha \cdot\left(p g^{-1} £_{\mathrm{R}} g+g^{-1} \phi g\right)\right] .
$$

So via the defining Hamiltonian relation in (2.3), the moment map $\mu$ for the action of $\mathcal{H}$ on $L \mathcal{O}_{\alpha}$ is given up to a constant by

$$
\langle\mu,(p, \phi, a)\rangle=-\oint_{C} \kappa \operatorname{Tr}\left[\alpha \cdot\left(p g^{-1} £_{\mathrm{R}} g+g^{-1} \phi g\right)\right] .
$$

We are left to check that the Hamiltonian condition in (2.4) is obeyed for $\mu$ in (4.90). This condition ultimately fixes the arbitrary constant that could otherwise appear in (4.90) to be zero. So we must verify that the expression for $\mu$ in (4.90) satisfies

$$
\begin{aligned}
\{\langle\mu,(p, \phi, a)\rangle,\langle\mu,(q, \psi, b)\rangle\} & =\langle\mu,[(p, \phi, a),(q, \psi, b)]\rangle \\
& =\left\langle\mu,\left(0,[\phi, \psi]+p £_{\mathrm{R}} \psi-q £_{\mathrm{R}} \phi, c(\phi, \psi)\right)\right\rangle .
\end{aligned}
$$

Here in the second line of (4.91), we recall from Section 3.3 the explicit form for the bracket on the Lie algebra of $\mathcal{H}$.

Before we perform any computations, let us make one observation. A noteworthy feature of the moment map in (4.90) is that $\mu$ vanishes when contracted with the central generator $(0,0, a)$ in the Lie algebra of $\mathcal{H}$. As a result, the cocycle $c(\phi, \psi)$ can effectively be set to zero when checking (4.91). The Hamiltonian condition for the Poisson bracket associated to generators of the form $(0, \phi, 0)$ and $(0, \psi, 0)$ then follows just as it does for the moment map (4.18) on the finite-dimensional orbit $\mathcal{O}_{\alpha}$.

Otherwise, the only non-trivial Poisson bracket to check is the one below,

$$
\begin{aligned}
\{\langle\mu,(p, 0,0)\rangle,\langle\mu,(0, \psi, 0)\rangle\} & =\Upsilon_{\alpha}\left(p £_{\mathrm{R}} g,\left.\psi\right|_{C} \cdot g\right), \\
& =p \oint_{C} \kappa \operatorname{Tr}\left(g^{-1} £_{\mathrm{R}} g,\left[\alpha, g^{-1} \psi g\right]\right), \\
& =p \oint_{C} \kappa \operatorname{Tr}\left(\alpha \cdot\left[g^{-1} \psi g, g^{-1} £_{\mathrm{R}} g\right]\right) .
\end{aligned}
$$

To simplify the last line in (4.92), we observe that

$$
£_{\mathrm{R}}\left(g^{-1} \psi g\right)=\left[g^{-1} \psi g, g^{-1} £_{\mathrm{R}} g\right]+g^{-1} £_{\mathrm{R}} \psi g .
$$


"ATMP-17-1-A1-BEA" — 2013/5/15 — 19:32 — page $65-\# 65$

Applying the identity in (4.93) to (4.92) and integrating by parts, we obtain

$$
\begin{aligned}
\{\langle\mu,(p, 0,0)\rangle,\langle\mu,(0, \psi, 0)\rangle\} & =-p \oint_{C} \kappa \operatorname{Tr}\left[\alpha \cdot\left(g^{-1} £_{\mathrm{R}} \psi g\right)\right] \\
& =\left\langle\mu,\left(0, p £_{\mathrm{R}} \psi, 0\right)\right\rangle
\end{aligned}
$$

as required by (4.91). Thus the action of $\mathcal{H}$ on $L \mathcal{O}_{\alpha}$ is Hamiltonian with moment map $\mu$ in (4.90).

\subsection{2 $L \mathcal{O}_{\alpha}$ as a coadjoint orbit of $\mathcal{H}$}

At this point, an excellent question to ask is why $L \mathcal{O}_{\alpha}$ should even admit a Hamiltonian action by $\mathcal{H}$. After all, the fact that $\mathcal{H}$ acts in a Hamiltonian fashion on $\overline{\mathcal{A}}$ is not obvious, given that the appearance of $\mathcal{H}$ itself is rather unexpected.

A bit more abstractly, if we are given a connected Lie group $H$, we can ask which symplectic spaces admit a Hamiltonian action by $H$. As we have already discussed, the coadjoint orbits of $H$ in the dual $\mathfrak{h}^{*}$ of its Lie algebra furnish canonical examples of such symplectic spaces. Thus, the simplest way to explain why $\mathcal{H}$ should act in Hamiltonian fashion on $L \mathcal{O}_{\alpha}$ is to identify $L \mathcal{O}_{\alpha}$ with a particular coadjoint orbit in the dual $\operatorname{Lie}(\mathcal{H})^{*}$ of the Lie algebra of $\mathcal{H}$.

To establish the interpretation of $L \mathcal{O}_{\alpha}$ as a coadjoint orbit, we first observe that central elements in $\mathcal{H}$ of course act trivially on the Lie algebra and therefore on its dual $\operatorname{Lie}(\mathcal{H})^{*}$. So for the purpose of discussing coadjoint orbits of $\mathcal{H}$, we need only consider the orbits in $\operatorname{Lie}(\mathcal{H})^{*}$ of the quotient group

$$
\overline{\mathcal{H}}=\mathcal{H} / U(1)_{\mathrm{Z}}=U(1)_{\mathrm{R}} \ltimes \mathcal{G}_{0} .
$$

We now claim that $L \mathcal{O}_{\alpha}$ can be formally identified as the orbit of $\overline{\mathcal{H}}$ which passes through the element $\gamma_{0} \in \operatorname{Lie}(\mathcal{H})^{*}$ defined by the pairing

$$
\left\langle\gamma_{0},(p, \phi, a)\right\rangle=-\int_{M} \kappa \wedge \delta_{C} \operatorname{Tr}(\alpha \phi)=-\oint_{C} \kappa \operatorname{Tr}(\alpha \phi) .
$$

Equivalently, in coordinates dual to $(p, \phi, a)$, we write

$$
\gamma_{0}=\left(0, \alpha \kappa \wedge \delta_{C}, 0\right) \text {. }
$$

Here, we regard $\alpha \kappa \wedge \delta_{C}$ as a section of the bundle $\Omega_{M}^{3} \otimes \mathfrak{g}$ of adjoint-valued three-forms on $M$ and hence as an element in the dual of the Lie algebra of 
the group $\mathcal{G}_{0}$, which is the identity component of the group $\mathcal{G}$ of all gauge transformations on $M$.

Let us consider the action of $\overline{\mathcal{H}}$ on $\gamma_{0}$. Since both $\kappa$ and $\delta_{C}$ are invariant under $U(1)_{R}, \gamma_{0}$ is fixed under $U(1)_{R}$. Hence, the orbit of the semidirect product $\overline{\mathcal{H}}=U(1)_{\mathrm{R}} \ltimes \mathcal{G}_{0}$ through $\gamma_{0}$ further reduces to the orbit of $\mathcal{G}_{0}$ through $\gamma_{0}$.

In general, elements of the group $\mathcal{G}$ of gauge transformations are described geometrically by maps from $M$ to $G$, and elements in the identity component $\mathcal{G}_{0}$ correspond to those maps $f: M \rightarrow G$, which are homotopically trivial (and hence can be continuously connected to the identity). Since $\gamma_{0}$ has delta-function support along $C, f$ acts on $\gamma_{0}$ by restriction to $C$ and so determines a point in $L \mathcal{O}_{\alpha}$. Upon setting $g=\left.f\right|_{C}$, the point is simply $g \alpha g^{-1} \in L \mathcal{O}_{\alpha}$. Conversely, if $g \in L G$ represents the point $g \alpha g^{-1} \in L \mathcal{O}_{\alpha}$, then $g$ can always be extended over $M$ to some homotopically trivial $f .{ }^{18}$ Hence the orbit of $\mathcal{G}_{0}$ through $\gamma_{0}$ is the loopspace $L \mathcal{O}_{\alpha}$.

Once $L \mathcal{O}_{\alpha}$ is identified as the coadjoint orbit of $\mathcal{H}$ through the element $\gamma_{0}$ in (4.97), $\Upsilon_{\alpha}$ immediately becomes the canonical coadjoint symplectic form. In precise analogy to (4.14) and (4.15), $\Upsilon_{\alpha}$ also derives from a pre-symplectic one-form $\Xi_{\alpha}$,

$$
\Upsilon_{\alpha}=\delta \Xi_{\alpha}
$$

where

$$
\Xi_{\alpha}=\oint_{C} \kappa \operatorname{Tr}\left(\alpha \cdot g^{-1} \delta g\right)
$$

Consequently, the moment map $\mu$ in (4.90) is given just as in (4.18) by the contraction

$$
\mu=-\iota_{V(p, \phi, a)} \Xi_{\alpha}
$$

where $V(p, \phi, a)$ is the vector field on $L \mathcal{O}_{\alpha}$ appearing in (4.86).

\footnotetext{
${ }^{18}$ By standard obstruction theory, $g: C \rightarrow G$ can always be extended from $C$ to $M$, so we need only argue that the extension can be chosen to be homotopically trivial. With our assumption that $G$ is compact, connected, simply connected, and simple, homotopy classes of maps $f: M \rightarrow G$ are represented by elements in $H^{3}(M ; \mathbb{Z})$, and homotopy classes of maps $\widetilde{f}: M \rightarrow G$ such that $\left.\widetilde{f}\right|_{C}=1$ are represented by elements in the relative group $H^{3}(M, C ; \mathbb{Z})$. But $H^{3}(M, C ; \mathbb{Z}) \cong H^{3}(M ; \mathbb{Z}) \cong \mathbb{Z}$ by the usual exact sequence. Hence if $f$ happens to be a homotopically non-trivial extension of $g$, we can always find another map $\widetilde{f}: M \rightarrow G$ such that $\left.\widetilde{f}\right|_{C}=1$ and the product $\widetilde{f} \cdot f$ (taken pointwise on $M$ ) is a homotopically trivial extension of $g$.
} 
As a coadjoint orbit of $\mathcal{H}$, the loopspace $L \mathcal{O}_{\alpha}$ also carries an invariant complex structure which is compatible with the symplectic form $\Upsilon_{\alpha}$, in the sense that together these data determine an invariant Kähler metric on $L \mathcal{O}_{\alpha}$. Concretely, the invariant complex structure on $L \mathcal{O}_{\alpha}$ is inherited from the corresponding complex structure $\mathcal{J}$ on $\mathcal{O}_{\alpha}$, such that the complex structure on $L \mathcal{O}_{\alpha}$ is given by the pointwise action of $\mathcal{J}$ on sections of $U^{*}\left(T \mathcal{O}_{\alpha}\right)$.

Since the symplectic form $\Upsilon_{\alpha}$ on $L \mathcal{O}_{\alpha}$ is also induced pointwise from the coadjoint symplectic form $\nu_{\alpha}$ on $\mathcal{O}_{\alpha}$, the invariant Kähler metric on $L \mathcal{O}_{\alpha}$ is then given by the natural pairing

$$
(\eta, \xi)=\oint_{C} \kappa \nu_{\alpha}(\eta, \mathcal{J} \cdot \xi)
$$

Again, $\eta$ and $\xi$ are sections of $U^{*}\left(T \mathcal{O}_{\alpha}\right)$ representing tangent vectors to $L \mathcal{O}_{\alpha}$. Of course, the metric in (4.101) is nothing more than the usual sigma model metric derived from the invariant Kähler metric on $\mathcal{O}_{\alpha}$ itself. As a formal consequence, the Riemannian path integral measure $\mathcal{D} U$ associated to the sigma model metric in (4.101) can be identified with the symplectic measure on $L \mathcal{O}_{\alpha}$ induced from $\Upsilon_{\alpha}$,

$$
\mathcal{D} U=\exp \left(\Upsilon_{\alpha}\right)
$$

By the preceding observations, the shift-invariant path integral describing $Z(\epsilon ; C, R)$ in (4.79) becomes a symplectic integral over $\overline{\mathcal{A}}_{\alpha}=\overline{\mathcal{A}} \times \epsilon L \mathcal{O}_{\alpha}$,

$$
Z(\epsilon ; C, R)=\frac{1}{\operatorname{Vol}(\mathcal{G})}\left(\frac{-\mathrm{i}}{2 \pi \epsilon}\right)^{\Delta_{\mathcal{G}} / 2} \int_{\overline{\mathcal{A}}_{\alpha}} \exp \left[\Omega_{\alpha}+\frac{\mathrm{i}}{2 \epsilon} S(A, U)\right]
$$

where we introduce the total symplectic form

$$
\Omega_{\alpha}=\Omega+\epsilon \Upsilon_{\alpha}
$$

Here $\Omega$ is the symplectic form on $\overline{\mathcal{A}}$ in (3.20), and in passing from (4.79) to (4.103), we have been careful to recall that the Kähler metric on $\epsilon L \mathcal{O}_{\alpha}$ is scaled by $\epsilon$ relative to the metric on $L \mathcal{O}_{\alpha}$ in (4.101). Hence, a crucial factor of $\epsilon$ multiplies $\Upsilon_{\alpha}$ in (4.104). The need for this factor will become clear momentarily.

\subsubsection{The action $S(A, U)$ as the square of the moment map}

We are left to show that the shift-invariant Seifert loop action $S(A, U)$ in (4.80) is precisely the square of the moment map for the Hamiltonian 
action of $\mathcal{H}$ on $\overline{\mathcal{A}}_{\alpha}$. Given the corresponding result (3.48) for the shiftinvariant action $S(A)$, this claim is not so unexpected, but it remains (at least to me) a fairly miraculous statement.

At this stage, we can explain the fundamental reason for the relative factor of $\epsilon$ appearing in the symplectic form $\Omega_{\alpha}$ in (4.104). With this factor, the moment map which describes the Hamiltonian action of $\mathcal{H}$ on the product $\overline{\mathcal{A}}_{\alpha}=\overline{\mathcal{A}} \times \epsilon L \mathcal{O}_{\alpha}$ is the sum of the moment map for $\overline{\mathcal{A}}$ in (3.41) with $\epsilon$ times the moment map for $L \mathcal{O}_{\alpha}$ in (4.90), so that the total moment map on $\overline{\mathcal{A}}_{\alpha}$ is given by

$$
\begin{aligned}
\langle\mu,(p, \phi, a)\rangle= & a-p \int_{M} \kappa \wedge \operatorname{Tr}\left[\frac{1}{2} £_{\mathrm{R}} A \wedge A+\epsilon \alpha\left(g^{-1} £_{\mathrm{R}} g\right) \delta_{C}\right] \\
& -\int_{M} \kappa \wedge \operatorname{Tr}\left(\phi \mathcal{F}_{A}\right)+\int_{M} d \kappa \wedge \operatorname{Tr}(\phi A),
\end{aligned}
$$

where

$$
\mathcal{F}_{A}=F_{A}+\epsilon\left(g \alpha g^{-1}\right) \delta_{C} .
$$

Again, $\mathcal{F}_{A}$ is the generalized curvature (4.78) appearing already in the shiftinvariant action $S(A, U)$. Indeed, we were careful to arrange for the factor of $\epsilon$ in (4.104) to ensure the appearance of $\mathcal{F}_{A}$ in (4.105).

As in Section 3, we proceed by computing directly the square of $\mu$ in (4.105). From the description of the invariant form on the Lie algebra of $\mathcal{H}$ in (3.38), we see that

$$
\begin{aligned}
(\mu, \mu)= & \int_{M} \kappa \wedge \operatorname{Tr}\left[£_{\mathrm{R}} A \wedge A+2 \epsilon \alpha\left(g^{-1} £_{\mathrm{R}} g\right) \delta_{C}\right] \\
& -\int_{M} \kappa \wedge d \kappa \operatorname{Tr}\left[\left(\frac{\kappa \wedge \mathcal{F}_{A}-d \kappa \wedge A}{\kappa \wedge d \kappa}\right)^{2}\right] .
\end{aligned}
$$

To simplify (4.107), let us expand the last term therein as

$$
\begin{aligned}
\int_{M} \kappa \wedge d \kappa \operatorname{Tr}\left[\left(\frac{\kappa \wedge \mathcal{F}_{A}-d \kappa \wedge A}{\kappa \wedge d \kappa}\right)^{2}\right] \\
=\int_{M} \frac{1}{\kappa \wedge d \kappa} \operatorname{Tr}\left[\left(\kappa \wedge \mathcal{F}_{A}\right)^{2}-2\left(\kappa \wedge \mathcal{F}_{A}\right)(d \kappa \wedge A)+(d \kappa \wedge A)^{2}\right] .
\end{aligned}
$$

The term in (4.108) that is quadratic in $\mathcal{F}_{A}$ appears explicitly in $S(A, U)$, and as for the term linear in $\mathcal{F}_{A}$, we need only extract the new contribution 
from the Seifert loop operator,

$$
-2 \epsilon \int_{M} \kappa \wedge \delta_{C} \operatorname{Tr}\left[\left(g \alpha g^{-1}\right)\left(\frac{d \kappa \wedge A}{d \kappa \wedge \kappa}\right)\right]=-2 \epsilon \oint_{C} \kappa \operatorname{Tr}\left[\alpha \cdot\left(g^{-1} \iota_{\mathrm{R}} A g\right)\right] .
$$

Here, we have applied the identity in (3.45).

After a little bit of algebra, we thus rewrite $(\mu, \mu)$ using (4.109) as

$$
\begin{aligned}
(\mu, \mu)= & \int_{M} \kappa \wedge \operatorname{Tr}\left(£_{\mathrm{R}} A \wedge A\right)+2 \int_{M} \kappa \wedge \operatorname{Tr}\left[\left(\iota_{\mathrm{R}} A\right) F_{A}\right] \\
& -\int_{M} \kappa \wedge d \kappa \operatorname{Tr}\left[\left(\iota_{\mathrm{R}} A\right)^{2}\right] \\
& +2 \epsilon \oint_{C} \kappa \operatorname{Tr}\left[\alpha \cdot\left(g^{-1} £_{\mathrm{R}} g+g^{-1} \iota_{\mathrm{R}} A g\right)\right] \\
& -\int_{M} \frac{1}{\kappa \wedge d \kappa} \operatorname{Tr}\left[\left(\kappa \wedge \mathcal{F}_{A}\right)^{2}\right] .
\end{aligned}
$$

At this stage, we apply our baroque identity in (3.47) to recognize the first line in (4.110) as the Chern-Simons action $\operatorname{CS}(A)$. We also have the much more transparent identity

$$
\operatorname{cs}_{\alpha}\left(U ;\left.A\right|_{C}\right)=\oint_{C} \operatorname{Tr}\left(\alpha \cdot g^{-1} d_{A} g\right)=\oint_{C} \kappa \operatorname{Tr}\left[\alpha \cdot\left(g^{-1} £_{\mathrm{R}} g+g^{-1} \iota_{\mathrm{R}} A g\right)\right] .
$$

The identity in (4.111) follows immediately if we recall that the vector field $\mathrm{R}$ is tangent to $C$ and satisfies $\langle\kappa, \mathrm{R}\rangle=1$.

So from (3.47), (4.110), and (4.111), we finally obtain the beautiful result

$$
\begin{aligned}
(\mu, \mu) & =\operatorname{Cs}(A)+2 \epsilon \operatorname{cs}_{\alpha}\left(U ;\left.A\right|_{C}\right)-\int_{M} \frac{1}{\kappa \wedge d \kappa} \operatorname{Tr}\left[\left(\kappa \wedge \mathcal{F}_{A}\right)^{2}\right] \\
& =S(A, U)
\end{aligned}
$$

Consequently the Seifert loop path integral in (4.103) assumes the canonical symplectic form required for non-abelian localization,

$$
Z(\epsilon ; C, R)=\frac{1}{\operatorname{Vol}(\mathcal{G})}\left(\frac{-\mathrm{i}}{2 \pi \epsilon}\right)^{\Delta_{\mathcal{G}} / 2} \int_{\overline{\mathcal{A}}_{\alpha}} \exp \left[\Omega_{\alpha}+\frac{\mathrm{i}}{2 \epsilon}(\mu, \mu)\right] .
$$




\subsubsection{Extension to multiple Seifert loop operators}

Although for simplicity we have focused throughout on the case of a single Seifert loop operator, the preceding discussion extends immediately to the case of multiple Seifert loop operators in $M$.

To state the general result, we let $C_{\ell}$ for $\ell=1, \ldots, \mathrm{L}$ be a set of disjoint Seifert fibers of $M$, each fiber labelled by an irreducible representation $R_{\ell}$ with highest weight $\alpha_{\ell}$. We then consider the symplectic space

$$
\overline{\mathcal{A}}_{\alpha}=\overline{\mathcal{A}} \times \epsilon L \mathcal{O}_{\alpha_{1}} \times \cdots \times \epsilon L \mathcal{O}_{\alpha_{\mathrm{L}}}
$$

with symplectic form

$$
\Omega_{\alpha}=\Omega+\epsilon \sum_{\ell=1}^{\mathrm{L}} \Upsilon_{\alpha_{\ell}}
$$

where $\boldsymbol{\alpha}=\left(\alpha_{1}, \ldots, \alpha_{\mathrm{L}}\right)$ serves as a multi-index.

The group $\mathcal{H}=U(1)_{\mathrm{R}} \ltimes \widetilde{\mathcal{G}}_{0}$ now acts on $\overline{\mathcal{A}}_{\boldsymbol{\alpha}}$ in a Hamiltonian fashion with moment map

$$
\begin{aligned}
\langle\mu,(p, \phi, a)\rangle & =a-p \int_{M} \kappa \wedge \operatorname{Tr}\left(\frac{1}{2} £_{\mathrm{R}} A \wedge A+\epsilon \sum_{\ell=1}^{\mathrm{L}}\left[\alpha\left(g^{-1} £_{\mathrm{R}} g\right) \delta_{C}\right]_{\ell}\right) \\
& -\int_{M} \kappa \wedge \operatorname{Tr}\left(\phi \mathcal{F}_{A}\right)+\int_{M} d \kappa \wedge \operatorname{Tr}(\phi A),
\end{aligned}
$$

where

$$
\mathcal{F}_{A}=F_{A}+\epsilon \sum_{\ell=1}^{\mathrm{L}}\left[\left(g \alpha g^{-1}\right) \delta_{C}\right]_{\ell} .
$$

Once more, to suppress the proliferation of subscripts, the index ' $\ell$ ' applies simultaneously to all quantities in brackets.

By the same calculations leading to (4.112), the shift-invariant action $S\left(A, U_{1}, \ldots, U_{\mathrm{L}}\right)$ in (4.83) is precisely the square of the moment map (4.116) for the Hamiltonian action of $\mathcal{H}$ on $\overline{\mathcal{A}}_{\alpha}$. So when applied to multiple Seifert 
"ATMP-17-1-A1-BEA" — 2013/5/15 — 19:32 — page $71-\# 71$

loop operators, the shift-invariant path integral in (4.82) can also be rewritten in the canonical symplectic form,

$$
\begin{aligned}
& Z\left(\epsilon ;\left(C_{1}, R_{1}\right), \ldots,\left(C_{\mathrm{L}}, R_{\mathrm{L}}\right)\right) \\
& \quad=\frac{1}{\operatorname{Vol}(\mathcal{G})}\left(\frac{-\mathrm{i}}{2 \pi \epsilon}\right)^{\Delta_{\mathcal{G}} / 2} \int_{\overline{\mathcal{A}}_{\boldsymbol{\alpha}}} \exp \left[\Omega_{\alpha}+\frac{\mathrm{i}}{2 \epsilon}(\mu, \mu)\right] .
\end{aligned}
$$

\section{Monodromy operators in two-dimensional Yang-Mills theory}

We began in Section 2 by recalling the well-known symplectic description for the Yang-Mills partition function, which we then extended in Section 3 to the partition function of Chern-Simons theory on a Seifert manifold. In this light, given the symplectic description for the Seifert loop operator in Section 4, one might ask which operator, if any, in two-dimensional YangMills theory plays a symplectic role analogous to that of the Seifert loop operator in Chern-Simons theory.

The answer to the preceding question is well-known, at least to aficionados of two-dimensional Yang-Mills theory, and it will be important when we perform localization computations for Seifert loop operators in Section 7. In a nutshell, the analogue on $\Sigma$ of the Seifert loop operator in $M$ is a local "monodromy" operator which inserts a singularity into the gauge field $A$ at a marked point of $\Sigma$. These monodromy operators were introduced by Witten [106] to model the current algebra vertex operators which describe a Wilson line puncturing $\Sigma$ in the canonical quantization of Chern-Simons theory on $\Sigma \times \mathbb{R}$, and in that context they were given a beautiful symplectic interpretation by Atiyah (see Section 5.2 of [10]). Although much of the following material is standard, our goal at present is thus to review a few essential ideas about monodromy operators in two-dimensional Yang-Mills theory.

The monodromy operator in Yang-Mills theory on $\Sigma$ is perhaps the simplest example disorder operator in gauge theory. By this statement, we mean that the monodromy operator is defined not in terms of a classical, gauge-invariant functional of $A$, as for instance we originally defined the Wilson loop operator in (1.4), but as a prescription to perform the twodimensional Yang-Mills path integral (2.1) over connections with specified classical singularities. Here by a 'classical' singularity, we mean a singularity that can appear in a solution to the Yang-Mills equations on $\Sigma$. Only for 
such singularities does one obtain a sensible path integral in the presence of the corresponding disorder operator.

The classical singularity which defines the monodromy operator in twodimensional Yang-Mills theory will simply be the reduction to $\Sigma$ of the classical singularity which appears in the Chern-Simons gauge field in the background of a Seifert loop operator. According to (4.65), the ChernSimons gauge field behaves classically near a Wilson loop wrapping $C \subset M$ as

$$
A=-\frac{\alpha}{k} d \varphi+V_{0} d \tau,
$$

at least up to gauge transformations. In comparison to (4.65), we have set $\epsilon=2 \pi / k$ and $U_{0}=\alpha$ without loss; $V_{0}$ is then any element in $\mathfrak{g}$ commuting with $\alpha$.

To reduce (5.1) to two dimensions, we take $p \in \Sigma$ be the basepoint of $C$, now a Seifert fiber of $M$, around which $(r, \varphi)$ serve as local polar coordinates. We also introduce a parameter $\lambda \in \mathfrak{t}$ to play the role of the ratio $\alpha / k$. In particular, despite the fact that in three dimensions $\alpha \in \Gamma_{\mathrm{wt}}$ is quantized as a weight of $G$, in two dimensions we allow $\lambda$ to vary continuously in $\mathfrak{t}$. The monodromy operator $\mathrm{V}_{\lambda}(p)$ is then defined as the disorder operator in two-dimensional Yang-Mills theory which creates a singularity in $A$ at the point $p$ of the form

$$
A=-\lambda d \varphi, \quad \lambda \in \mathfrak{t},
$$

again up to gauge transformations. Of course, in reducing to two dimensions, we omit the component of $A$ proportional to $d \tau$ in (5.1).

Let us make two elementary comments about (5.2). First, because the connection in (5.2) is flat away from $p$, it trivially satisfies the classical YangMills equation $d_{A} \star F_{A}=0$ on a punctured neighborhood of $p$. Second, as our terminology for $\mathrm{V}_{\lambda}(p)$ suggests, the connection in (5.2) has non-trivial monodromy around $p$ given by

$$
\Lambda=\exp (2 \pi \lambda) .
$$

The parameter $\lambda$ appears with a positive sign in (5.3) since the holonomy of $A$ is defined with a negative sign, as in (4.69).

Before we proceed further, we need to consider how the operator $\mathrm{V}_{\lambda}(p)$ depends upon the parameter $\lambda$. For instance, even after we fix the maximal 
"ATMP-17-1-A1-BEA" — 2013/5/15 - 19:32 — page 73 — \#73

torus $T \subset G$, the Weyl group $\mathfrak{W}$ of $G$ remains as a residual discrete symmetry acting on $\lambda$.

Somewhat less obviously, as pointed out by Gukov and Witten [70] in relation to surface operators in four-dimensional gauge theory (for which the same codimension two singularity in $A$ plays an essential role), the monodromy operator $\mathrm{V}_{\lambda}(p)$ is also invariant under any shift of the form $\lambda \mapsto \lambda+y$, where $y \in \mathfrak{t}$ satisfies the integrality condition $\exp (2 \pi y)=1$. This shift in $\lambda$ leaves the monodromy $\Lambda$ in (5.3) invariant and is induced by a singular gauge transformation generated locally at $p$ by the $T$-valued function

$$
(r, \varphi) \longmapsto \exp (\varphi y) .
$$

Intrinsically as in Section 4.1, $y$ is characterized as an element of the cocharacter lattice

$$
\Gamma_{\text {cochar }}=\operatorname{Hom}(U(1), T),
$$

which becomes isomorphic to the coroot lattice $\Gamma_{\text {cort }}$ when $G$ is simplyconnected. In the present discussion of two-dimensional Yang-Mills theory, we will not always assume $G$ to be simply-connected, so we are careful to distinguish $\Gamma_{\text {cort }}$ and $\Gamma_{\text {cochar }}$. Thus if we consider both the action of the Weyl group $\mathfrak{W}$ on $\lambda$ as well as shifts $\lambda \mapsto \lambda+y$ generated by the singular gauge transformations in (5.4), the gauge-invariant label for the monodromy operator $\mathrm{V}_{\lambda}(p)$ is not the parameter $\lambda \in \mathfrak{t}$ per se but rather the image of $\lambda$ in the quotient $\mathfrak{t} / \mathfrak{W}_{\text {aff }}$, where $\mathfrak{W}_{\text {aff }}$ is the affine Weyl group of $G$,

$$
\mathfrak{W}_{\text {aff }}=\mathfrak{W} \ltimes \Gamma_{\text {cochar }} .
$$

The fact that the monodromy operator is actually labelled by elements of the quotient $\mathfrak{t} / \mathfrak{W}_{\text {aff }}$, as opposed to elements of $\mathfrak{t}$, has a natural geometric interpretation. Because the maximal torus $T$ of $G$ can itself be presented as $T=\mathfrak{t} / \Gamma_{\text {cochar }}$, we identify $\mathfrak{t} / \mathfrak{W}_{\text {aff }} \cong T / \mathfrak{W}$. On the other hand, points in $T / \mathfrak{W}$ correspond to conjugacy classes in $G$. As a result, the monodromy operator $\mathrm{V}_{\lambda}(p)$ is naturally labelled in a gauge-invariant fashion by the conjugacy class $\mathfrak{C}_{\lambda}=\mathrm{Cl}[\Lambda]$ containing the monodromy $\Lambda=\exp (2 \pi \lambda)$.

\section{Hamiltonian interpretation}

As an aside, the monodromy operators that create singularities in $A$ of the form (5.2) are well-known in two-dimensional Yang-Mills theory, but 
they are often described in a slightly different way. Instead of working on the punctured Riemann surface $\Sigma^{o}=\Sigma-\{p\}$ as we have done so far, we consider a small disc $D \subset \Sigma$ containing the point $p$, and we replace the local operator $\mathrm{V}_{\lambda}(p)$ by an external state $\left|\mathfrak{C}_{\lambda}\right\rangle$ in the Hilbert space constructed by quantizing Yang-Mills theory on the circle bounding $D$. Tautologically, the state $\left|\mathfrak{C}_{\lambda}\right\rangle$ is obtained by performing the path integral for Yang-Mills theory on $D$ with the monodromy operator inserted at $p$. But because twodimensional Yang-Mills theory is such a simple theory, the state $\left|\mathfrak{C}_{\lambda}\right\rangle$ can be given an absolutely explicit description, which was applied by Witten in [106] to perform exact computations with the monodromy operator in the Hamiltonian formulation of two-dimensional Yang-Mills theory.

Very briefly, to describe the state $\left|\mathfrak{C}_{\lambda}\right\rangle$, we recall that the only gaugeinvariant data carried by a connection on $S^{1}$ is the conjugacy class of its holonomy $W$ as an element of $G$,

$$
W=P \exp \left(-\oint_{S^{1}} A\right) \in G
$$

Hence, the Hilbert space $\mathcal{H}$ of two-dimensional Yang-Mills theory on the disc $D$ is the space of square-integrable class functions $\Psi(W)$ on $G$. By the PeterWeyl theorem, $\mathcal{H}=L^{2}(G)^{G}$ is spanned by the characters of the irreducible representations $R$ of $G$, so the Yang-Mills Hilbert space can be presented in a basis of states $|R\rangle$ corresponding to each irreducible representation of $G$.

By definition, the monodromy operator $\mathrm{V}_{\lambda}(p)$ enforces the condition that $A$ have holonomy around the boundary of $D$ which lies in the conjugacy class $\mathfrak{C}_{\lambda}=\mathrm{Cl}[\Lambda]$. Thus, as a formal class function on $G$, the corresponding external state $\left|\mathfrak{C}_{\lambda}\right\rangle$ must be a delta-function supported on $\mathfrak{C}_{\lambda}$. Concretely, via the standard orthonormality of characters, the state $\left|\mathfrak{C}_{\lambda}\right\rangle$ can be expanded in the basis of representations as

$$
\left|\mathfrak{C}_{\lambda}\right\rangle=\sum_{R}|R\rangle\left\langle R \mid \mathfrak{C}_{\lambda}\right\rangle=\sum_{R} \overline{\operatorname{ch}_{R}\left(\mathfrak{C}_{\lambda}\right)}|R\rangle
$$

Here $\operatorname{ch}_{R}$ is the character associated to the representation $R$, and $\left\langle\mathfrak{C}_{\lambda} \mid R\right\rangle=$ $\operatorname{ch}_{R}\left(\mathfrak{C}_{\lambda}\right)$ by definition. ${ }^{19}$ Among other advantages, the description of $\left|\mathfrak{C}_{\lambda}\right\rangle$ in (5.8) makes manifest the fact that the monodromy operator $\boldsymbol{V}_{\lambda}(p)$ depends only upon the gauge-invariant data of the conjugacy class $\mathfrak{C}_{\lambda}$, or equivalently upon the point in the quotient $\mathfrak{t} / \mathfrak{W}_{\text {aff }}$, and not upon the particular representative $\lambda \in \mathfrak{t}$ introduced initially.

\footnotetext{
${ }^{19}$ As standard, $\left\langle R \mid \mathfrak{C}_{\lambda}\right\rangle$ is the complex conjugate of $\left\langle\mathfrak{C}_{\lambda} \mid R\right\rangle$, accounting for appearance of $\overline{\operatorname{ch}_{R}\left(\mathfrak{C}_{\lambda}\right)}$ in (5.8).
} 
"ATMP-17-1-A1-BEA" — 2013/5/15 - 19:32 — page $75-\# 75$

Actually, as noted by Witten in [106], the normalization of the deltafunction associated to $\left|\mathfrak{C}_{\lambda}\right\rangle$ is subtle, since this delta-function depends upon the choice of a measure on the space of conjugacy classes of $G$. We have made a particular choice in (5.8), but another choice would multiply $\left|\mathfrak{C}_{\lambda}\right\rangle$ by an arbitrary class function on $G$. Equivalently, we have not been very careful so far to fix the absolute normalization of $\mathrm{V}_{\lambda}(p)$. In this paper, we implicitly normalize $V_{\lambda}(p)$ through its symplectic path integral, to which we now turn.

\subsection{The monodromy operator path integral as a symplectic integral}

Although the monodromy operator in two-dimensional Yang-Mills theory is perhaps interesting in its own right as a very simple, almost pedagogical, example of a disorder operator in gauge theory, our interest in the monodromy operator stems from the fact that it admits a symplectic description precisely analogous to that established for the Seifert loop operator in Section 4.3. The essentials of the symplectic description for $\mathrm{V}_{\lambda}(p)$ were explained long ago by Atiyah in Section 5.2 of his beautiful lectures [10] on Chern-Simons theory, which we follow shamelessly here.

Let us first introduce the monodromy operator path integral,

$$
\begin{aligned}
Z(\epsilon ; p, \lambda)= & \frac{1}{\operatorname{Vol}(\mathcal{G}(P))}\left(\frac{1}{2 \pi \epsilon}\right)^{\Delta_{\mathcal{G}(P)} / 2} \\
& \times \int_{\mathcal{A}(P)} \mathcal{D} A \vee_{\lambda}(p) \exp \left[\frac{1}{2 \epsilon} \int_{\Sigma} \operatorname{Tr}\left(F_{A} \wedge \star F_{A}\right)\right]
\end{aligned}
$$

By definition, $Z(\epsilon ; p, \lambda)$ is now a path integral over connections on $\Sigma$ with a singularity at $p$ of the form (5.2), up to gauge transformations. Though we suppress analytic details, see for instance $[30,63]$ for rigorous models of the relevant spaces of singular connections associated to the monodromy operator. Nonetheless, one small analytic detail concerning (5.9) will be important. In our previous discussion of the monodromy operator, we emphasized that $\mathrm{V}_{\lambda}(p)$ depends on the parameter $\lambda \in \mathfrak{t}$ only up to the action of the affine Weyl group $\mathfrak{W}_{\text {aff. }}$. Yet to discuss the path integral in (5.9), especially in its symplectic incarnation, we find it necessary to fix at the outset a particular value for $\lambda$ in its orbit under $\mathfrak{W}_{\text {aff }}$.

To explain why fixing the value of $\lambda$ under the action of $\mathfrak{W}_{\text {aff }}$ is necessary, let us reconsider the geometric meaning of $\lambda$ in Yang-Mills theory. We 
began in Section 2 with a given principal $G$-bundle $P$ over $\Sigma$, on which $A$ is a connection. However, as noted by Gukov and Witten [70] in the same situation, when $A$ has a singularity at a point $p \in \Sigma$, the $G$-bundle $P$ is only naturally defined on the punctured Riemann surface $\Sigma^{o}=\Sigma-\{p\}$. We are free to extend $P$ over the puncture at $p$, but there is no natural way to do so. Instead, different extensions of $P$ are labelled by the various ways to lift the monodromy $\Lambda$ as an element of the torus $T \cong \mathfrak{t} / \Gamma_{\text {cochar }}$ to a corresponding Lie algebra element $\lambda \in \mathfrak{t}$, and these extensions are all related by the singular gauge transformations in (5.4).

To give a well-known example, let us assume that the Yang-Mills gauge group $G$ takes the adjoint form $G=\widetilde{G} / \mathcal{Z}(\widetilde{G})$, where $\widetilde{G}$ is simply-connected and $\mathcal{Z}(\widetilde{G})$ is the center of $\widetilde{G}$. In this case, the extension of $P$ even as a smooth $G$-bundle over $p$ is not unique. Rather, the extension depends upon the choice of a characteristic class $\zeta \in \mathcal{Z}(\widetilde{G})$, where $\zeta$ represents the possible monodromy in $\widetilde{G}$ that obstructs $P$ from extending smoothly as a $\widetilde{G}$-bundle over $p$. (Since $\zeta$ becomes trivial once we pass from $\widetilde{G}$ to $G$, the principal bundle $P$ does extend smoothly as a $\widetilde{G}$-bundle.) If $y \in \Gamma_{\text {cochar }}$ is an element of $\mathfrak{t}$ satisfying $1 \neq \exp (2 \pi y) \in \mathcal{Z}(\widetilde{G})$, the corresponding singular gauge transformation in (5.4) shifts the value of $\zeta$ and therefore changes the topology of $P$.

As usual in Yang-Mills theory, the need to choose a particular extension of $P$ over the puncture at $p$ and hence a particular lift from $\Lambda$ to $\lambda$ can be obviated by summing over all such choices. For instance, when $G$ is the adjoint form of a simply connected group $\widetilde{G}$, one computes the physical Yang-Mills partition function by summing over all topological types of $P$. Nevertheless, from the purely semi-classical perspective here, the Yang-Mills path integral is most naturally defined for a fixed $G$-bundle $P$ and hence, in the case of (5.9), for a particular choice of $\lambda$ in its orbit under $\mathfrak{W}_{\text {aff }}$.

Fixing the residual action of $\mathfrak{W}_{\text {aff }}$ on $\lambda$ has two important consequences. First, once $P$ and $\lambda$ are fixed, the group $\mathcal{G}(P)$ of gauge transformations appearing in (5.9) consists of those gauge transformations, which are strictly non-singular at $p$, precisely as in Section 2. Singular gauge transformations at $p$, such as those in (5.4), would otherwise shift $\lambda$. Second, once the extension of $P$ over $p$ is chosen, the curvature of the singular connection in (5.2) is well-defined at $p$ and can be evaluated as

$$
F_{A}=-2 \pi \lambda \delta_{p}
$$

Here, $\delta_{p}$ is a two-form on $\Sigma$ with delta-function support which represents the Poincaré dual of $p$, and the formula for the curvature $F_{A}$ in (5.10) follows 
"ATMP-17-1-A1-BEA" — 2013/5/15 - 19:32 — page 77 — \#77

from the local description (5.2) of $A$ via the naive relation $d(d \varphi)=2 \pi \delta_{p}$. Note that under shifts of $\lambda$ generated by gauge transformations that are singular at $p$, the curvature $F_{A}$ in (5.10) also shifts, so that the formula in (5.10) is really only sensible once $\lambda$ is fixed. Not surprisingly, the delta-function curvature of $F_{A}$ at $p$ will be an important ingredient in the symplectic description of the monodromy operator.

Although the particular choice for $\lambda$ will not matter until Section 5.2, for concreteness let us make that choice now. In specifying a distinguished representative for $\lambda$ under the action of $\mathfrak{W}_{\text {aff }}$, we assume for convenience that the Yang-Mills gauge group $G=\widetilde{G}$ is simply-connected, with an eye towards the eventual application to Chern-Simons theory. As explained for instance in Ch. 5 of [94], the Cartan subalgebra of $G$ then divides into a countable set of alcoves under the action of $\mathfrak{W}_{\text {aff }}$, and each alcove serves as a fundamental domain for $\mathfrak{W}_{\text {aff }}$. So to make a particular choice for $\lambda$ in its orbit under $\mathfrak{W}_{\text {aff }}$, we simply pick a distinguished Weyl alcove $\mathrm{D}_{+}$, in which we assume $\lambda$ lies.

Following the discussion in Section 4.1, for which $\lambda \geq 0$ by convention, we take the distinguished Weyl alcove $\mathrm{D}_{+} \subset \mathrm{C}_{+}$to sit in the positive Weyl chamber of $\boldsymbol{t}$. According to (4.20), the positive Weyl chamber $\mathrm{C}_{+}$is a simplicial cone, bounded by walls associated to the positive simple roots of $G$. At the tip of this cone sits a unique alcove containing the origin in $\mathfrak{t}$, and we take $D_{+}$to be that alcove. Explicitly, $D_{+}$is the simplex in $C_{+}$for which $\lambda \geq 0$ satisfies the additional bound

$$
\langle\vartheta, \lambda\rangle \leq 1
$$

where $\vartheta$ is the highest root of $G$. Stated more geometrically, for each orbit of $\mathfrak{W}_{\text {aff }}$ in $\mathfrak{t}$, we take $\lambda \in \mathrm{D}_{+}$to be the positive representative which lies at minimal distance from the origin.

Since these conventions may seem a little bit abstract, let us give a concrete example, corresponding to the case $G=S U(r+1)$. The Cartan subalgebra is then represented by diagonal matrices of the form $i \operatorname{diag}\left(\lambda_{1}, \ldots\right.$, $\left.\lambda_{r+1}\right)$ such that $\lambda_{1}+\cdots+\lambda_{r+1}=0$. A standard set of positive simple roots for $S U(r+1)$ is given by the successive differences $\lambda_{j}-\lambda_{j+1}$ for $j=1, \ldots, r$, so the positive Weyl chamber is described by the inequalities $\lambda_{1} \geq \lambda_{2} \geq \cdots \geq \lambda_{r+1}$. Finally, the highest root $\vartheta$ of $S U(r+1)$ is given by the difference $\lambda_{1}-\lambda_{r+1}$, so the fundamental Weyl alcove $\mathrm{D}_{+}$is described by the additional constraint $\lambda_{1}-\lambda_{r+1} \leq 1$. For instance, if $G=S U(2)$, then $\lambda_{1}$ lies in the interval $\mathrm{D}_{+}=\left[0, \frac{1}{2}\right]$. 
"ATMP-17-1-A1-BEA" — 2013/5/15 - 19:32 — page 78 — \#8

\section{Monodromy operators and coadjoint orbits}

One satisfying aspect of our work in Section 4.3 is that the Seifert loop path integral in its symplectic form appears as an elegant extension of the path integral which describes the Chern-Simons partition function on $M$. To describe the Seifert loop operator symplectically, we merely replace the symplectic space $\overline{\mathcal{A}}$ with the product $\overline{\mathcal{A}}_{\alpha}=\overline{\mathcal{A}} \times \epsilon L \mathcal{O}_{\alpha}$, where the loopspace $L \mathcal{O}_{\alpha}$ can be considered as a coadjoint orbit for the Hamiltonian group $\mathcal{H}$ that acts on $\overline{\mathcal{A}}$.

To recast the monodromy operator path integral (5.9) as a symplectic path integral of the canonical form, we proceed in complete analogy to the case of the Seifert loop operator. As we reviewed in Section 2, the canonical symplectic integral that describes the basic Yang-Mills partition function on $\Sigma$ is determined by the Hamiltonian action of the group $\mathcal{G}(P)$ on the affine space $\mathcal{A}(P)$. To describe the monodromy operator by analogy to the Seifert loop operator, we just consider the product of $\mathcal{A}(P)$ with an appropriate coadjoint orbit of $\mathcal{G}(P)$.

In fact, the correct coadjoint orbit is easy to guess. Under reduction from $C \subset M$ to $p \in \Sigma$, the analogue of the loopspace $\epsilon L \mathcal{O}_{\alpha}$ is the finitedimensional orbit $\mathcal{O}_{\lambda}$ itself, and $\mathcal{O}_{\lambda}$ can be immediately embedded as a coadjoint orbit of $\mathcal{G}(P)$. Briefly, we recall that the dual of the Lie algebra of $\mathcal{G}(P)$ is formally the space of sections of the bundle $\Omega_{\Sigma}^{2} \otimes \operatorname{ad}(P)$. We then regard $\mathcal{O}_{\lambda}$ as the orbit of $\mathcal{G}(P)$ passing through the singular section of $\Omega_{\Sigma}^{2} \otimes \operatorname{ad}(P)$ which is given by

$$
\gamma_{0}=\lambda \delta_{p}
$$

in complete analogy to (4.97).

Since $\gamma_{0}$ has delta-function support at $p$, an element of $\mathcal{G}(P)$ acts on $\mathcal{O}_{\lambda}$ by restriction to the point $p$. Hence, if we endow $\mathcal{O}_{\lambda}$ with the coadjoint symplectic form $\nu_{\lambda}$, the action of $\mathcal{G}(P)$ on $\mathcal{O}_{\lambda}$ is Hamiltonian with moment map

$$
\langle\mu, \phi\rangle=-\int_{\Sigma} \delta_{p} \operatorname{Tr}\left[\left(g \lambda g^{-1}\right) \cdot \phi\right]=-\operatorname{Tr}\left[\left.\left(g \lambda g^{-1}\right) \cdot \phi\right|_{p}\right]
$$

Here, $\phi$ transforms as a section of the bundle $\operatorname{ad}(P)$ on $\Sigma$, and $g$ is an element of $G$, which we use to specify the point $g \lambda g^{-1}$ on $\mathcal{O}_{\lambda}$. We obtain the final expression in (5.13) by performing the integral over $\Sigma$ using the delta-function, thereby restricting $\phi$ to $p$. Clearly (5.13) then agrees with the moment map (4.18) for the action of $G$ on $\mathcal{O}_{\lambda}$. 
"ATMP-17-1-A1-BEA" — 2013/5/15 — 19:32 — page 79 — \#79

To recast the monodromy operator path integral as a symplectic path integral of the canonical form, we consider the product

$$
\mathcal{A}(P)_{\lambda}=\mathcal{A}(P) \times 2 \pi \mathcal{O}_{\lambda}
$$

with symplectic form

$$
\Omega_{\lambda}=\Omega+2 \pi \nu_{\lambda}
$$

Here, $\Omega$ is the symplectic form (2.2) on the affine space $\mathcal{A}(P)$ of connections, and the factors of $2 \pi$ in (5.14) and (5.15) are ultimately necessary to agree with our conventions for $\mathrm{V}_{\lambda}(p)$ in (5.2).

As in Section 4.3, we consider the diagonal action of $\mathcal{G}(P)$ on $\mathcal{A}(P)_{\lambda}$. The moment map for this action is of course the sum of the moment maps, which describe the action of $\mathcal{G}(P)$ on each factor in (5.14). Accounting for the coefficient of $2 \pi$ in (5.15), we thus write the total moment map for the action of $\mathcal{G}(P)$ on $\mathcal{A}(P)_{\lambda}$ as

$$
\langle\mu, \phi\rangle=-\int_{\Sigma} \operatorname{Tr}\left(\mathcal{F}_{A} \phi\right) .
$$

Here, $\mathcal{F}_{A}$ is a generalized curvature on $\Sigma$, which includes the effective deltafunction contribution at $p$ from (5.13),

$$
\mathcal{F}_{A}=F_{A}+2 \pi\left(g \lambda g^{-1}\right) \delta_{p}
$$

Based on (5.16) and (5.17), the action $S=\frac{1}{2}(\mu, \mu)$ for the canonical symplectic integral over $\mathcal{A}(P)_{\lambda}$ then takes precisely the same form as the Yang-Mills action, but expressed in terms of $\mathcal{F}_{A}$,

$$
S=\frac{1}{2}(\mu, \mu)=-\frac{1}{2} \int_{\Sigma} \operatorname{Tr}\left(\mathcal{F}_{A} \wedge \star \mathcal{F}_{A}\right) .
$$

With (5.18) in hand, we now claim that the monodromy operator path integral can be interpreted as the canonical symplectic integral determined by the Hamiltonian action of $\mathcal{G}(P)$ on $\mathcal{A}(P)_{\lambda}$,

$$
Z(\epsilon ; p, \lambda)=\frac{1}{\operatorname{Vol}(\mathcal{G}(P))}\left(\frac{1}{2 \pi \epsilon}\right)^{\Delta_{\mathcal{G}(P)} / 2} \int_{\mathcal{A}(P)_{\lambda}} \exp \left[\Omega_{\lambda}-\frac{1}{2 \epsilon}(\mu, \mu)\right] .
$$

To relate (5.19) to the starting path integral in (5.9), let us consider carefully the generalized Yang-Mills action in (5.18). Unlike the usual YangMills action for $F_{A}$, which is a perfectly well-behaved functional of smooth 
"ATMP-17-1-A1-BEA" — 2013/5/15 - 19:32 — page 80 — \#80

connections on $\Sigma$, the Yang-Mills action for $\mathcal{F}_{A}$ is badly divergent for smooth configurations of $A$, due to the fact that it implicitly involves the square of a delta-function. Since the Yang-Mills action is also positive-definite, the only connections on $\Sigma$ for which the action in (5.18) is finite are those for which $A$ has the appropriate singularity to cancel the explicit delta-function curvature in $\mathcal{F}_{A}$. Of course, because of the exponential suppression in the integrand of (5.19), the path integral only receives contributions from connections with finite action. Thus, all non-zero contributions to the symplectic integral over $\mathcal{A}(P)_{\lambda}$ arise from singular connections on $\Sigma$ whose curvatures near $p$ take the form

$$
F_{A}=-2 \pi\left(g \lambda g^{-1}\right) \delta_{p}+\cdots,
$$

where the "..." indicate terms in $F_{A}$ which are regular at $p$. Comparing (5.10) to (5.20), we see that the singular connections that define the monodromy operator $\mathrm{V}_{\lambda}(p)$ are precisely those which contribute to the symplectic path integral in (5.19).

To place (5.19) into its proper physical context, we note that disorder operators in quantum field theory can often be described in terms of an auxiliary defect theory living on the submanifold in spacetime where the operator is inserted. In this language, the path integral in (5.19) describes the monodromy operator inserted at $p$ via a very simple defect theory, one which incorporates only finitely-many degrees of freedom valued in $\mathcal{O}_{\lambda}$.

In Section 7.3, at the very end of the paper, we will revisit the symplectic interpretation of the monodromy operator $\mathrm{V}_{\lambda}(p)$ in Yang-Mills theory on $\Sigma$.

\subsection{More about the classical monodromy operator}

So far, we have described the monodromy operator $\mathrm{V}_{\lambda}(p)$ as a quantum operator in two-dimensional Yang-Mills theory. In preparation for the localization computations in Section 7 , we now wish to consider $V_{\lambda}(p)$ in somewhat more detail from a purely classical perspective, as reflected in the structure of the moduli space of Yang-Mills solutions with monodromy on $\Sigma$.

Actually, we restrict attention throughout to only the most basic solutions of Yang-Mills theory in the presence of the monodromy operator. Namely, we consider connections which are flat on the punctured Riemann surface $\Sigma^{o}=\Sigma-\{p\}$ and otherwise have a singularity at $p$ of the form (5.2), up to gauge transformations. For such connections, $\mathcal{F}_{A}=0$ everywhere on $\Sigma$, so these solutions make the dominant contribution to the monodromy operator path integral. 
"ATMP-17-1-A1-BEA" — 2013/5/15 - 19:32 — page 81 — \#1

LOCALIZATION FOR WILSON LOOPS IN CHERN-SIMONS

By analogy to the Wilson loop moduli space $\mathcal{M}(C, \alpha)$ introduced in Section 4.2 , we let $\mathcal{N}(P ; p, \lambda)$ denote the moduli space of flat connections on $\Sigma$ with a singularity at $p$ of the form (5.2), up to gauge transformations. Here we are careful to keep track of the data for both the principal $G$-bundle $P$ over $\Sigma$ and the point $p$ at which the operator $\mathrm{V}_{\lambda}$ is inserted. However, because the topology of $\mathcal{N}(P ; p, \lambda) \equiv \mathcal{N}(P ; \lambda)$ does not vary with the continuous choice of $p$, we frequently omit $p$ from the notation.

In the special case $\lambda=0$, the extended moduli space $\mathcal{N}(P ; \lambda)$ reduces to the moduli space $\mathcal{N}(P)$ of non-singular flat connections on $\Sigma$. Yet even when $\lambda>0$ is non-zero, the extended moduli space $\mathcal{N}(P ; \lambda)$ is still related to $\mathcal{N}(P)$ in two essential ways. First, $\mathcal{N}(P ; \lambda)$ is the total space of a natural symplectic fibration over $\mathcal{N}(P)$. Second, $\mathcal{N}(P ; \lambda)$ is the splitting manifold for the universal bundle on $\mathcal{N}(P)$ (when that universal bundle exists). These statements are entirely standard, but since they both feature heavily in the cohomological computations in Section 7.3, we take some time to review them now.

\section{$\mathcal{N}(P ; \lambda)$ as a moduli space of homomorphisms}

To start, let us describe $\mathcal{N}(P ; \lambda)$ very concretely as a moduli space of homomorphisms. We proceed in complete analogy to our discussion of the classical Wilson loop moduli space $\mathcal{M}(C, \alpha)$ in Section 4.2.

As well-known, if the Yang-Mills gauge group $G=\widetilde{G}$ is simply-connected and $P$ is topologically trivial, the moduli space $\mathcal{N}(P)$ of (non-singular) flat connections appears directly as the moduli space of homomorphisms $\varrho: \pi_{1}(\Sigma) \rightarrow G$, where the fundamental group $\pi_{1}(\Sigma)$ is generated by elements $\mathrm{a}_{\ell}$ and $\mathrm{b}_{\ell}$ for $\ell=1, \ldots, h$, subject to the single relation

$$
\prod_{\ell=1}^{h}\left[a_{\ell}, b_{\ell}\right]=1, \quad\left[a_{\ell}, b_{\ell}\right] \equiv a_{\ell} b_{\ell} a_{\ell}^{-1} b_{\ell}^{-1} .
$$

Here $h$ is the genus of $\Sigma$.

By essentially the same observations as in Section 4.2, the extended moduli space $\mathcal{N}(P ; \lambda)$ can then be presented as the moduli space of pairs $\left(\varrho^{\circ}, U_{0}\right)$, where $\varrho^{o}$ is now a homomorphism from the fundamental group of the punctured Riemann surface $\Sigma^{o}=\Sigma-\{p\}$ to $G$,

$$
\varrho^{o}: \pi_{1}\left(\Sigma^{o}\right) \longrightarrow G,
$$

and $U_{0}=g \lambda g^{-1}$ is an element of $\mathcal{O}_{\lambda}$ related to $\varrho^{o}$ by

$$
\varrho^{o}(\mathrm{c})=\exp \left(2 \pi U_{0}\right) \text {. }
$$


Here $\mathrm{c} \in \pi_{1}\left(\Sigma^{o}\right)$ is the distinguished element which represents the small onecycle about $p$ parameterized by $\varphi$ in (5.2), so that

$$
\prod_{\ell=1}^{h}\left[\mathrm{a}_{\ell}, \mathrm{b}_{\ell}\right]=\mathrm{c}
$$

and the role of $U_{0}$ is to encode the monodromy of the connection at $p$.

In discussing Yang-Mills theory on $\Sigma$, we do not necessarily wish to assume that the gauge group $G$ is simply-connected nor that the bundle $P$ is trivial. So more generally, we take $G$ to be the quotient of its simply connected cover $\widetilde{G}$ by a subgroup of the center $\mathcal{Z}(\widetilde{G})$. The topology of $P$ as a principal $G$-bundle over $\Sigma$ is then encoded by an element $\zeta \in \mathcal{Z}(\widetilde{G})$. The central element $\zeta$ represents the possible monodromy in $\widetilde{G}$ at a generic point $q \in \Sigma$, which otherwise obstructs $P$ from extending smoothly as a $\widetilde{G}$-bundle over $q$.

To incorporate the topology of $P$ into the general description of $\mathcal{N}(P ; \lambda)$, we introduce the doubly-punctured Riemann surface $\Sigma^{o o}$,

$$
\Sigma^{o o}=\Sigma-\{p\}-\{q\}
$$

As usual, the fundamental group $\pi_{1}\left(\Sigma^{o o}\right)$ is generated by elements $\mathrm{a}_{\ell}, \mathrm{b}_{\ell}$, $\mathrm{c}$, and $\mathrm{d}$ for $\ell=1, \ldots, h$, subject to the doubly extended relation

$$
\prod_{\ell=1}^{h}\left[a_{\ell}, b_{\ell}\right]=\mathrm{cd} .
$$

Here, $\mathrm{c}$ and $\mathrm{d}$ represent small, suitably-oriented one-cycles about the respective punctures at $p$ and $q$.

To describe flat connections on $P$ with monodromy, we consider homomorphisms $\varrho^{O O}$ from the fundamental group $\pi_{1}\left(\Sigma^{o o}\right)$ to the simply connected group $\widetilde{G}$,

$$
\varrho^{o o}: \pi_{1}\left(\Sigma^{o o}\right) \longrightarrow \widetilde{G}
$$

such that $\varrho^{O O}$ satisfies

$$
\varrho^{o O}(\mathrm{c})=\exp \left(2 \pi U_{0}\right), \quad \varrho^{o O}(\mathrm{~d})=\zeta .
$$


As before, $U_{0}$ is an element of $\mathcal{O}_{\lambda}$ encoding the monodromy at $p$, and in the special case that $\lambda=U_{0}=0$, the homomorphism $\varrho^{o O}$ in (5.28) describes a non-singular flat connection on the $G$-bundle $P$.

By way of notation, we let $\widetilde{\mathcal{N}}(P ; \lambda)$ be the space of pairs $\left(\varrho^{o o}, U_{0}\right)$ satisfying (5.28), modulo the diagonal action of $\widetilde{G}$,

$$
\widetilde{\mathcal{N}}(P ; \lambda)=\left\{\left(\varrho^{o O}, U_{0}\right) \mid \varrho^{O O}(\mathrm{c})=\exp \left(2 \pi U_{0}\right), \varrho^{O O}(\mathrm{~d})=\zeta\right\} / \widetilde{G} .
$$

Although $U_{0}$ will be useful to have around in a moment, if $\lambda$ is generic we can solve for $U_{0}$ in terms of $\varrho^{o O}(\mathrm{c})$ via (5.28), so that in terms of $\varrho^{o O}$ alone,

$$
\begin{aligned}
\widetilde{\mathcal{N}}(P ; \lambda) & =\left\{\varrho^{o o} \mid \varrho^{o o}(\mathrm{c}) \in \mathfrak{C}_{\lambda}, \varrho^{o o}(\mathrm{~d})=\zeta\right\} / \widetilde{G}, \\
\mathfrak{C}_{\lambda} & =\operatorname{Cl}[\exp (2 \pi \lambda)] .
\end{aligned}
$$

The description of $\widetilde{\mathcal{N}}(P ; \lambda)$ here is entirely analogous to the description of $\mathcal{M}(C, \alpha)$ in (4.72). Moreover, when we specialize in Section 7.1 to the case that $M$ is a Seifert manifold, we will make the relationship between $\tilde{\mathcal{N}}(P ; \lambda)$ and $\mathcal{M}(C, \alpha)$ even more precise.

The moduli space $\tilde{\mathcal{N}}(P ; \lambda)$ of homomorphisms in (5.29) is almost, but not quite, $\mathcal{N}(P ; \lambda)$. Rather, $\widetilde{\mathcal{N}}(P ; \lambda)$ is an unramified cover of $\mathcal{N}(P ; \lambda)$ of degree $|\widetilde{G}: G|^{2 h}$, where $|\widetilde{G}: G|$ is the order of the basic covering $\widetilde{G} \rightarrow G$. For instance, if $G=\widetilde{G} / \mathcal{Z}(\widetilde{G})$ is the adjoint form of $\widetilde{G}$, then $|\widetilde{G}: G|=|\mathcal{Z}(\widetilde{G})|$. We obtain a covering of $\mathcal{N}(P ; \lambda)$ in (5.29) because the holonomies associated to the cycles $\mathrm{a}_{\ell}$ and $\mathrm{b}_{\ell}$ are specified as elements of $\widetilde{G}$, not $G$, in (5.27). This caveat aside, the distinction between $\widetilde{\mathcal{N}}(P ; \lambda)$ and $\mathcal{N}(P ; \lambda)$ will at most affect an overall numerical factor in the cohomological formulae in Section 7.3 and is otherwise inessential.

\section{$\mathcal{N}(P ; \lambda)$ as a symplectic fibration over $\mathcal{N}(P)$}

From the perspective of the present paper, we now arrive at the first important geometric fact about $\mathcal{N}(P ; \lambda)$. As we have already mentioned, in the trivial case that $\lambda=0, \mathcal{N}(P ; \lambda)$ immediately reduces to the moduli space $\mathcal{N}(P)$ of non-singular flat connections on $\Sigma$. More generally if $\lambda$ is nonzero but small, in a sense to be made precise, then $\mathcal{N}(P ; \lambda)$ is still related to $\mathcal{N}(P)$ in a simple way, and this relationship underlies the cohomological interpretation for both the monodromy and the Seifert loop operators.

Specifically, when $\lambda \geq 0$ obeys the strict bound $\langle\vartheta, \lambda\rangle<1$ in (5.11) and $\mathcal{N}(P)$ itself is non-singular, the extended moduli space $\mathcal{N}(P ; \lambda)$ fibers 
$[30,43,63]$ smoothly over $\mathcal{N}(P)$

$$
2 \pi \mathcal{O}_{-\lambda} \longrightarrow \mathcal{N}(P ; \lambda)
$$

Here the fiber of $\mathcal{N}(P ; \lambda)$ is the coadjoint orbit of $G$ through $-2 \pi \lambda$, as indicated by the prefactor and the sign in (5.31). Of course, at the level of topology, $2 \pi \mathcal{O}_{-\lambda}$ is indistinguishable from the basic orbit $\mathcal{O}_{\lambda}$, but $2 \pi \mathcal{O}_{-\lambda}$ carries the symplectic form $-2 \pi \nu_{\lambda}$.

The symplectic structure on the fiber of (5.31) is relevant, because $\mathcal{N}(P)$ and $\mathcal{N}(P ; \lambda)$ also carry natural symplectic forms. These moduli spaces are determined by the vanishing of the respective moment maps $\mu=F_{A}$ and $\mu=\mathcal{F}_{A}$ for the Hamiltonian action of $\mathcal{G}(P)$ on the symplectic spaces $\mathcal{A}(P)$ and $\mathcal{A}(P)_{\lambda}$, from which $\mathcal{N}(P)$ and $\mathcal{N}(P ; \lambda)$ inherit symplectic forms under the symplectic quotient construction. Abusing notation somewhat, we let $\Omega$ be the symplectic form on $\mathcal{N}(P)$ inherited from the form (2.2) on $\mathcal{A}(P)$, and we let $\Omega_{\lambda}$ be the symplectic form on $\mathcal{N}(P ; \lambda)$ inherited from the corresponding form $(5.15)$ on $\mathcal{A}(P)_{\lambda}$.

The smooth fibration in (5.31) is now compatible with the symplectic data on $\mathcal{O}_{\lambda}, \mathcal{N}(P)$, and $\mathcal{N}(P ; \lambda)$ in the following sense. Again provided that $\lambda \geq 0$ satisfies the strict inequality $\langle\vartheta, \lambda\rangle<1$ in (5.11), the symplectic form $\Omega_{\lambda}$ on $\mathcal{N}(P ; \lambda)$ decomposes as a sum

$$
\Omega_{\lambda}=\mathrm{q}^{*} \Omega-2 \pi e_{\lambda} .
$$

Here, $\mathrm{q}^{*} \Omega$ is the pullback of the symplectic form $\Omega$ on $\mathcal{N}(P)$ to $\mathcal{N}(P ; \lambda)$, and $\boldsymbol{e}_{\lambda}$ is a closed two-form on $\mathcal{N}(P ; \lambda)$ which restricts fiberwise to the coadjoint symplectic form $\nu_{\lambda}$. The relative factor of $-2 \pi$ in (5.32) is responsible for the appearance of the same factor in (5.31).

Following the exposition in Section 3.5 of [70], let us quickly sketch how the fibration of $\mathcal{N}(P ; \lambda)$ arises. First, the fiber of $\mathcal{N}(P ; \lambda)$ is parameterized by the element $U_{0} \in \mathcal{O}_{\lambda}$ appearing in (5.29), which thereby determines the monodromy $\varrho^{o o}(\mathrm{c})$ around the puncture at $p$. Otherwise, the base of $\mathcal{N}(P ; \lambda)$ is parameterized by the holonomies associated to the remaining generators $\mathrm{a}_{\ell}$ and $\mathrm{b}_{\ell}$ of $\pi_{1}\left(\Sigma^{o o}\right)$. As $\varrho^{o o}$ is a group homomorphism, those holonomies necessarily satisfy

$$
\prod_{\ell=1}^{h}\left[\varrho^{o O}\left(\mathrm{a}_{\ell}\right), \varrho^{o o}\left(\mathrm{~b}_{\ell}\right)\right]=\zeta \cdot \exp \left(2 \pi U_{0}\right) .
$$


"ATMP-17-1-A1-BEA" — 2013/5/15 - 19:32 — page $85-\# 85$

For $\lambda=U_{0}=0$, a homomorphism $\varrho^{o o}$ satisfying (5.33) determines a nonsingular flat connection on the bundle $P$. So for $U_{0} \neq 0$ fixed and sufficiently small (we discuss the precise bound in a moment), the relation (5.33) is just a deformation of constraint defining $\mathcal{N}(P)$. Provided that the moduli space $\mathcal{N}(P)$ is smooth, its topology is unchanged under continuous deformations, so $\varrho^{O O}\left(\mathrm{a}_{\ell}\right)$ and $\varrho^{O O}\left(\mathrm{~b}_{\ell}\right)$ effectively parameterize a copy of $\mathcal{N}(P)$.

As one instance when the smoothness condition holds, we take $\Sigma$ to be a Riemann surface of genus $h \geq 1$. Then $\mathcal{N}(P)$ is smooth if $G=S U(r+1) /$ $\mathbb{Z}_{r+1}$ is the adjoint form of $S U(r+1)$, and $P$ is a topologically non-trivial $G$-bundle over $\Sigma$ characterized by a generator $\zeta$ of $\mathbb{Z}_{r+1}$. For example, if $G=S O(3), P$ is the $S O(3)$-bundle over $\Sigma$ with non-vanishing StiefelWhitney class $w_{2} \neq 0$.

At the level of topology, we have sketched why $\mathcal{N}(P ; \lambda)$ fibers over $\mathcal{N}(P)$, but the symplectic nature of the fibration in (5.31) is also extremely important. Indeed, the symplectic decomposition (5.32) of $\Omega_{\lambda}$ turns out to be a basic ingredient in our cohomological analysis of the Seifert loop operator in Section 7.3.

The formula (5.32) for $\Omega_{\lambda}$ can be understood in at least two ways. As exploited by Jeffrey [63], one way to understand (5.32) is as a general consequence of symplectic reduction at a non-zero value of the moment map. We have already noted that $\mathcal{N}(P ; \lambda)$ can be constructed as the quotient under $\mathcal{G}(P)$ of the vanishing locus for the moment map $\mu=\mathcal{F}_{A}$ in the product $\mathcal{A}(P)_{\lambda}=\mathcal{A}(P) \times 2 \pi \mathcal{O}_{\lambda}$. But since $\mathcal{F}_{A}=F_{A}+2 \pi\left(g \lambda g^{-1}\right) \delta_{p}$, the extended moduli space $\mathcal{N}(P ; \lambda)$ can be equivalently constructed as the quotient under $\mathcal{G}(P)$ of the locus in the original affine space $\mathcal{A}(P)$ where the moment map $\mu=F_{A}$ takes values in the coadjoint orbit through $-2 \pi \lambda \delta_{p}$. For $\lambda$ sufficiently small, the formula for $\Omega_{\lambda}$ in (5.32) then follows from general facts about symplectic reduction at a non-zero value of the moment map.

Alternatively, we can check the formula for $\Omega_{\lambda}$ in (5.32) directly, by evaluating $\Omega_{\lambda}$ on two tangent vectors to $\mathcal{N}(P ; \lambda)$. Concretely, if $\mathcal{X}$ is a tangent vector to $\mathcal{N}(P ; \lambda)$ at a point corresponding to a given flat connection $A$ with singularity at $p$ of the form (5.2), then $X$ can be represented as a sum

$$
X=\eta+d_{A} \phi .
$$

Here, $\eta$ is a smooth, non-singular section of $\Omega_{\Sigma}^{1} \otimes \operatorname{ad}(P)$ which satisfies $d_{A} \eta=d_{A}^{\dagger} \eta=0$, where $d_{A}^{\dagger}=-\star d_{A} \star$ is the adjoint of $d_{A}$. By the usual Hodge theory, $\eta$ thereby represents a tangent vector to the base $\mathcal{N}(P)$ in a local trivialization of the fibration in (5.31). 
Of course, we must allow $X$ to have a component along the fiber of $\mathcal{N}(P ; \lambda)$. For this reason, we have introduced besides $\eta$ a gauge-trivial term $d_{A} \phi$ in $X$, where $\phi$ is an arbitrary smooth section of $\operatorname{ad}(P)$. To explain the role of $\phi$, we recall that the fiber of $\mathcal{N}(P ; \lambda)$ parameterizes the possible monodromies of $A$ at $p$. Since non-trivial gauge transformations at $p$ act transitively upon the possible monodromies, $d_{A} \phi$ thus represents a tangent vector to the fiber in (5.31).

If $X_{1}$ and $X_{2}$ are two such tangent vectors to $\mathcal{N}(P ; \lambda)$, associated to pairs $(\eta, \phi)$ and $(\xi, \psi)$ as in (5.34), then the symplectic pairing on $\mathcal{N}(P ; \lambda)$ is given explicitly by

$$
\begin{aligned}
\Omega_{\lambda}\left(X_{1}, X_{2}\right) & =-\int_{\Sigma} \operatorname{Tr}\left(X_{1} \wedge X_{2}\right), \\
& =-\int_{\Sigma} \operatorname{Tr}\left[\left(\eta+d_{A} \phi\right) \wedge\left(\xi+d_{A} \psi\right)\right], \\
& =-\int_{\Sigma} \operatorname{Tr}(\eta \wedge \xi)-\int_{\Sigma} \operatorname{Tr}\left(d_{A} \phi \wedge d_{A} \psi\right) .
\end{aligned}
$$

In passing to the third line of (5.35), we have noted that the cross-terms otherwise appearing in the second line of (5.35) vanish upon integration by parts, since $d_{A} \eta=d_{A} \xi=0$. We then immediately recognize the pairing between $\eta$ and $\xi$ in (5.35) as the natural symplectic pairing on $\mathcal{N}(P)$,

$$
\Omega(\eta, \xi)=-\int_{\Sigma} \operatorname{Tr}(\eta \wedge \xi)
$$

We are left to consider the pairing between $\phi$ and $\psi$. By assumption, the background connection $A$ which represents a point in $\mathcal{N}(P ; \lambda)$ satisfies $d_{A}^{2}=F_{A}=-2 \pi \lambda \delta_{p}$. Integrating by parts, we find

$$
-\int_{\Sigma} \operatorname{Tr}\left(d_{A} \phi \wedge d_{A} \psi\right)=\int_{\Sigma} \operatorname{Tr}\left(\phi\left[F_{A}, \psi\right]\right)=-\left.2 \pi \operatorname{Tr}(\phi[\lambda, \psi])\right|_{p} .
$$

Comparing (5.37) to (4.15) and being careful about signs, we thus see that the restriction of $\Omega_{\lambda}$ to the fiber of $\mathcal{N}(P ; \lambda)$ is given by $-2 \pi \nu_{\lambda}$, as claimed in (5.32).

To finish up our discussion of the symplectic fibration in (5.31), let us quickly consider the regime in which the fibration is valid. As we have been careful to emphasize, the formula (5.32) for $\Omega_{\lambda}$ holds only when $\lambda \geq 0$ satisfies the strict bound $\langle\vartheta, \lambda\rangle<1$, where $\vartheta$ is the highest root of $G$. According to (5.11), this bound is saturated on the far wall of the Weyl alcove $D_{+}$, and the reader may wonder what happens to $\mathcal{N}(P ; \lambda)$ when $\lambda$ hits that 
"ATMP-17-1-A1-BEA" — 2013/5/15 — 19:32 — page 87 — \#87

wall. In brief, when $\lambda$ satisfies $\langle\vartheta, \lambda\rangle=1$, a cycle (generically, a two-cycle) collapses in $\mathcal{N}(P ; \lambda)$, and the symplectic form $\Omega_{\lambda}$ degenerates. ${ }^{20}$

The collapsing cycle in $\mathcal{N}(P ; \lambda)$ is not hard to see if we simply compare the two descriptions for $\mathcal{N}(P ; \lambda)$ in (5.29) and (5.30). In passing from (5.29) to $(5.30)$, we used the relation $\varrho^{o o}(\mathbf{c})=\exp \left(2 \pi U_{0}\right)$ to eliminate the parameter $U_{0} \in \mathcal{O}_{\lambda}$ in favor of the monodromy $\varrho^{o O}(\mathrm{c}) \in \mathfrak{C}_{\lambda}$. For generic values of $\lambda$, the element $U_{0}$ is determined uniquely by $\varrho^{o o}(\mathrm{c})$, and the conjugacy class $\mathfrak{C}_{\lambda}$ which parameterizes the monodromy is diffeomorphic to the coadjoint orbit $2 \pi \mathcal{O}_{\lambda}$, from which $\mathfrak{C}_{\lambda}$ inherits the natural symplectic form (up to sign). However, at exceptional values of $\lambda$, the relation between $\varrho^{o O}(\mathrm{c})$ and $U_{0}$ ceases to be invertible, and a non-trivial cycle in $2 \pi \mathcal{O}_{\lambda}$ collapses under the exponential map to $\mathfrak{C}_{\lambda}$. Since $\mathfrak{C}_{\lambda}$ is identified with the fiber of $\mathcal{N}(P ; \lambda)$, as we implicitly used in the gauge theory computation (5.35) of $\Omega_{\lambda}$, the same cycle collapses in $\mathcal{N}(P ; \lambda)$.

To present the collapsing cycle explicitly, we observe that both $\mathcal{O}_{\lambda}$ and $\mathfrak{C}_{\lambda}$ can be described as quotients

$$
\mathcal{O}_{\lambda}=G / G_{\lambda}, \quad \mathfrak{C}_{\lambda}=G / \mathcal{Z}_{\Lambda}
$$

where $G_{\lambda}$ is the stabilizer of $\lambda$ under the adjoint action of $G$, and $\mathcal{Z}_{\Lambda}$ is the centralizer of $\Lambda=\exp (2 \pi \lambda)$ in $G$. The stabilizer $G_{\lambda}$ is always a subgroup of the centralizer $\mathcal{Z}_{\Lambda}$, so the exponential map from $2 \pi \mathcal{O}_{\lambda}$ to $\mathfrak{C}_{\lambda}$ fits generally into a sequence

$$
\mathcal{Z}_{\Lambda} / G_{\lambda} \longrightarrow \underset{\substack{\downarrow \\ \mathfrak{C}_{\lambda}}}{2 \pi \mathcal{O}_{\lambda}}
$$

As apparent from (5.39), whenever $\mathcal{Z}_{\Lambda}$ is enhanced beyond $G_{\lambda}$, the exponential map from $2 \pi \mathcal{O}_{\lambda}$ to $\mathfrak{C}_{\lambda}$ is not smoothly invertible, and the cycle $\mathcal{Z}_{\Lambda} / G_{\lambda}$ collapses in $2 \pi \mathcal{O}_{\lambda}$.

The relevant criterion for the centralizer $\mathcal{Z}_{\Lambda}$ to enhance is well-known, so we will merely state it. See Section 7.4 of [72] for a textbook discussion. For convenience, we take $G$ to be simply connected. The fiber $\mathcal{Z}_{\Lambda} / G_{\lambda}$ in (5.39)

\footnotetext{
${ }^{20}$ Of course, along the walls of $\mathrm{D}_{+}$which coincide with the walls of the full Weyl chamber $\mathrm{C}_{+}$, cycles also collapse in $\mathcal{N}(P ; \lambda)$, for the basic reason that $\lambda$ ceases to be regular on those walls. Nonetheless, on the walls of $C_{+}$, the formula (5.32) for $\Omega_{\lambda}$ itself remains valid.
} 
“ATMP-17-1-A1-BEA" — 2013/5/15 - 19:32 — page 88 — \#8

is then non-trivial precisely when $\lambda$ satisfies the integrality condition

$$
\langle\beta, \lambda\rangle \in \mathbb{Z}-\{0\}
$$

for some root $\beta$ of $G$. In fact, because the roots of $G$ appear in positive and negative pairs $\pm \beta$, the condition (5.40) is always satisfied by $\lambda$ for an even number (possibly zero) of roots.

As an elementary example of the integrality condition (5.40), we consider the case $G=S U(2)$, for which we parameterize $\lambda \in \mathfrak{t}$ in terms of a real variable $x$ as $\lambda=i \operatorname{diag}(x,-x)$. If $x=0$, both $2 \pi \mathcal{O}_{\lambda}$ and $\mathfrak{C}_{\lambda}$ are points, and the exponential map is trivial. Similarly, if $x$ is generic, $2 \pi \mathcal{O}_{\lambda}$ and $\mathfrak{C}_{\lambda}$ are mutually diffeomorphic to the quotient $S U(2) / U(1)=S^{2}$. Finally, in the special case that $x=j / 2$ for some non-zero integer $j$, corresponding precisely to the integrality condition in $(5.40), 2 \pi \mathcal{O}_{\lambda}$ is again a two-sphere, but $\exp (2 \pi \lambda)= \pm 1$ is central in $S U(2)$, so $\mathfrak{C}_{\lambda}$ is a point.

For general $G$, the same pattern holds. Generically, if $\lambda$ satisfies the integrality condition in (5.40), it does so for a single pair of roots $\pm \beta$, and a two-cycle collapses under the exponential map to $\mathfrak{C}_{\lambda}$.

Returning to our analysis of $\mathcal{N}(P ; \lambda)$, we take $\lambda$ to sit in the fundamental Weyl alcove $\mathrm{D}_{+}$. Then as $\lambda$ moves away from the origin in $\mathrm{D}_{+}$, the integrality condition (5.40) is first satisfied when $\lambda$ hits the wall defined by the highest root $\vartheta$ of $G$, so that $\langle\vartheta, \lambda\rangle=1$. Along that wall, the gauge symmetry preserved by $\mathrm{V}_{\lambda}(p)$ enhances, and the cycle $\mathcal{Z}_{\Lambda} / G_{\lambda}$ collapses in the fiber of $\mathcal{N}(P ; \lambda)$.

\section{$\mathcal{N}(P ; \lambda)$ as a splitting manifold for the universal bundle}

Beyond its mere existence, the fibration $(5.31)$ of $\mathcal{N}(P ; \lambda)$ over $\mathcal{N}(P)$ plays an important theoretical role, since it presents $\mathcal{N}(P ; \lambda)$ as the splitting manifold for the universal bundle on $\mathcal{N}(P)$. This observation appears for instance as Proposition 3.5 in [63], where it is applied to a cohomological computation very similar to the one in Section 7.3, and it is what we wish to explain now.

We start by introducing the universal bundle $\mathcal{V}$ associated to the basic moduli space $\mathcal{N}(P)$. So far, we have interpreted $\mathcal{N}(P)$ as the moduli space of flat connections on the principal $G$-bundle $P$ over $\Sigma$. On the other hand, the classic theorem of Narasimhan and Seshadri [87] establishes a one-to-one correspondence between flat connections on $P$ and suitable holomorphic bundles over $\Sigma$, so that $\mathcal{N}(P)$ admits an algebraic description as well. For the eventual application in Section 7.3, we are most interested in the standard case that $G=S U(r+1) / \mathbb{Z}_{r+1}$ is the adjoint form of $\widetilde{G}=S U(r+1)$, and $P$ 
"ATMP-17-1-A1-BEA" — 2013/5/15 - 19:32 — page 89 — \#89

is a topologically non-trivial $G$-bundle over $\Sigma$ characterized by a generator $\zeta \in \mathbb{Z}_{r+1}$. If $\Sigma$ is a Riemann surface of genus $h \geq 1$, these assumptions ensure that $\mathcal{N}(P)$ is smooth. Then in its algebraic incarnation, $\mathcal{N}(P)$ is the moduli space of stable holomorphic vector bundles of rank $r+1$ on $\Sigma$, with first Chern class equal to $\zeta \bmod r+1$ and with fixed determinant.

In the situation above, the universal bundle $\mathcal{V}$ exists as a holomorphic vector bundle of rank $r+1$ over the product $\Sigma \times \mathcal{N}(P)$,

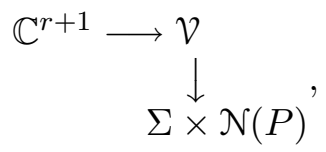

such that $\mathcal{V}$ possesses the following universal property. By definition, $\mathcal{N}(P)$ parameterizes holomorphic vector bundles of rank $r+1$ on $\Sigma$, so each point $y \in \mathcal{N}(P)$ determines a corresponding vector bundle $V_{y}$ on $\Sigma$. On the other hand, the restriction of $\mathcal{V}$ to $\Sigma \times\{y\}$ also determines a rank $r+1$ holomorphic vector bundle on $\Sigma$. According to the universal property of $\mathcal{V}$, these bundles are isomorphic,

$$
\left.V_{y} \cong \mathcal{V}\right|_{\Sigma \times\{y\}}, \quad y \in \mathcal{N}(P) .
$$

In general, the universal property does not determine $\mathcal{V}$ uniquely. For if a bundle $\mathcal{V}$ satisfies (5.42), then so does the tensor product of $\mathcal{V}$ with any holomorphic line bundle on $\Sigma \times \mathcal{N}(P)$ which is itself the pullback from a holomorphic line bundle on $\mathcal{N}(P)$. Nevertheless, this ambiguity in $\mathcal{V}$ can be fixed (see Section 9 of [12] for details), and a 'normalized' version of the universal bundle exists which is unique up to isomorphism. Although the details will not matter here, for concreteness we take $\mathcal{V}$ to be that normalized universal bundle.

Just as the restriction of $\mathcal{V}$ to $\Sigma \times\{y\}$ determines a holomorphic vector bundle on $\Sigma$ for each point $y \in \mathcal{N}(P)$, the restriction of $\mathcal{V}$ to $\{p\} \times \mathcal{N}(P)$ determines a holomorphic vector bundle on $\mathcal{N}(P)$ for each point $p \in \Sigma$. By way of abbreviation, we set

$$
\left.\mathcal{V}_{p} \equiv \mathcal{V}\right|_{\{p\} \times \mathcal{N}(P)} .
$$

Since $p$ varies continuously in $\Sigma$, the topology of $\mathcal{V}_{p}$ is independent of $p$. In particular, the Chern classes of $\mathcal{V}_{p}$ do not depend on the point $p$ and therefore provide distinguished elements in the integral cohomology ring of $\mathcal{N}(P)$.

Throughout the following discussion, we impose the crucial condition that $\lambda>0$ be regular. Given that condition, we now claim that $\mathcal{N}(P ; p, \lambda) \equiv$ $\mathcal{N}(P ; \lambda)$ is tautologically the splitting manifold for the vector bundle $\mathcal{V}_{p}$ 
over $\mathcal{N}(P)$. That is, under the map $\mathrm{q}: \mathcal{N}(P ; \lambda) \rightarrow \mathcal{N}(P)$ in the symplectic fibration (5.31), the pullback $\mathrm{q}^{*} \mathcal{V}_{p}$ splits smoothly into a direct sum of complex line bundles $\mathcal{L}_{j}$ for $j=1, \ldots, r+1$ over $\mathcal{N}(P ; \lambda)$,

$$
\mathrm{q}^{*} \mathcal{V}_{p} \cong \bigoplus_{j=1}^{r+1} \mathcal{L}_{j}
$$

Hence, the Chern classes of the line bundles $\mathcal{L}_{j}$ realize the Chern roots $\mathfrak{u}_{j}$ of $\mathcal{V}_{p}$

$$
\mathrm{q}^{*} c\left(\mathcal{V}_{p}\right)=\prod_{j=1}^{r+1}\left(1+\mathfrak{u}_{j}\right), \quad \mathfrak{u}_{j} \equiv c_{1}\left(\mathcal{L}_{j}\right) \in H^{2}(\mathcal{N}(P ; \lambda) ; \mathbb{Z})
$$

where $c\left(\mathcal{V}_{p}\right)$ is the total Chern class,

$$
c\left(\mathcal{V}_{p}\right)=1+c_{1}\left(\mathcal{V}_{p}\right)+\cdots+c_{r+1}\left(\mathcal{V}_{p}\right) .
$$

As apparent from (5.45) and essential later, any symmetric function of the Chern roots $\mathfrak{u}_{j}$ is the pullback from $\mathcal{N}(P)$ of an associated function of the Chern classes $c_{j}\left(\mathcal{V}_{p}\right)$.

The splitting in (5.44) can be understood from various perspectives. One approach is to interpret $\mathcal{N}(P ; \lambda)$ algebraically, via the classic theorem of Mehta and Seshadri [83], as a moduli space of holomorphic bundles on $\Sigma$ with parabolic structure at $p$. See Section 3.4 of [70] for an excellent review of parabolic bundles as they occur in gauge theory. From the parabolic perspective, $\mathcal{N}(P ; \lambda)$ is then precisely the splitting manifold constructed on general grounds in Ch. 21 of [25].

Rather than follow the algebraic route here, we will take an equivalent but slightly more hands-on approach, which allows us to relate the discussion to some ideas already appearing in Section 4.1. We begin by unraveling a few definitions.

Let us consider arbitrary points $y \in \mathcal{N}(P)$ and $z \in \mathcal{N}(P ; \lambda)$ satisfying $y=\mathrm{q}(z)$, so that $z$ is a point in the fiber of $\mathcal{N}(P ; \lambda)$ over $y$. Relative to a local trivialization of the fibration (5.31), we can always write $z=\left(U_{0}, y\right)$, where $U_{0} \in \mathcal{O}_{\lambda}$ parameterizes the coadjoint fiber over $y$.

Now by definition, the fiber of the pullback $\mathrm{q}^{*} \mathcal{V}_{p}$ over $z$ is the fiber of $\mathcal{V}_{p}$ itself over $y$. However, according to the universal property (5.42), the fiber 
"ATMP-17-1-A1-BEA" — 2013/5/15 — 19:32 — page 91 — \#91

of $\mathcal{V}_{p}$ over $y$ is isomorphic to the fiber of $V_{y}$ over $p$,

$$
\left.\mathcal{V}_{p}\right|_{y}=\left.\left.\mathcal{V}\right|_{\{p\} \times\{y\}} \cong V_{y}\right|_{p}, \quad\{p\} \times\{y\} \in \Sigma \times \mathcal{N}(P),
$$

where we recall that $V_{y}$ is the holomorphic vector bundle over $\Sigma$ determined by $y \in \mathcal{N}(P)$. The splitting in (5.44) is thus equivalent to a smooth splitting of $\left.V_{y}\right|_{p}$ for each $z=\left(U_{0}, y\right)$, such that the splitting respects the action of $G$ on $U_{0}$ induced by gauge transformations at $p \in \Sigma$.

To obtain the requisite splitting, we consider the infinitesimal action of $U_{0}$ on the fiber of $V_{y}$ at $p$. Here, we use the embedding $\mathcal{O}_{\lambda} \subset \mathfrak{g}$, where the Lie algebra $\mathfrak{g}=\mathfrak{s u}(r+1)$ acts via the standard, fundamental representation on the fiber $\left.V_{y}\right|_{p} \cong \mathbb{C}^{r+1}$. If $\lambda$ is regular, then $U_{0}$ acts on $\left.V_{y}\right|_{p}$ with distinct eigenvalues, and each eigenvalue is associated to a one-dimensional complex eigenspace $L_{j}$ for $j=1, \ldots, r+1$. Thus for each $U_{0} \in \mathcal{O}_{\lambda}$, the fiber of $V_{y}$ at $p$ decomposes into a sum of eigenspaces

$$
\left.V_{y}\right|_{p}=\bigoplus_{j=1}^{r+1} L_{j} .
$$

Moreover, the eigenspace decomposition (5.48) naturally respects the simultaneous action of $G$ (or more precisely, the universal cover $\widetilde{G}$ ) on both $U_{0}$ and $\left.V_{y}\right|_{p}$, so the decomposition does not depend on the choice of the local trivialization we used to write $z=\left(U_{0}, y\right)$.

As $z$ varies, the eigenspaces $L_{j}$ themselves vary smoothly as the fibers of corresponding complex line bundles $\mathcal{L}_{j}$ over $\mathcal{N}(P ; \lambda)$. Via (5.47) and (5.48), these line bundles then provide the tautological splitting (5.44) of $\mathrm{q}^{*} \mathcal{V}_{p}$.

Actually, we can go a bit further in identifying the line bundles $\mathcal{L}_{j}$ over $\mathcal{N}(P ; \lambda)$. By construction, each $\mathcal{L}_{j}$ respects the action of $G$ on the coadjoint fibers of $\mathcal{N}(P ; \lambda)$, so $\mathcal{L}_{j}$ must restrict fiberwise to a homogeneous line bundle. That is,

$$
\left.\mathcal{L}_{j}\right|_{\mathcal{O}_{\lambda}}=\mathfrak{L}\left(-\alpha_{j}\right), \quad \alpha_{j} \in \Gamma_{\mathrm{wt}},
$$

where $\mathfrak{L}\left(-\alpha_{j}\right)$ is the homogeneous line bundle on $\mathcal{O}_{\lambda}$ determined by an appropriate weight $\alpha_{j}$ of $S U(r+1)$. The slightly perverse sign in (5.49) becomes useful in a moment. Implicit in (5.49), as the fiber of $\mathcal{N}(P ; \lambda)$ varies continuously over $\mathcal{N}(P)$, the particular weight $\alpha_{j}$ associated to each line bundle $\mathcal{L}_{j}$ does not jump. Thus, the tautological splitting (5.44) is characterized by a finite set of weights $\left\{\alpha_{1}, \ldots, \alpha_{r+1}\right\}$, which we would like to determine. 
To do so, we once again unravel definitions. As in Section 4.1, to discuss homogeneous line bundles on $\mathcal{O}_{\lambda}$, we take $G=S U(r+1)$ to be simply connected for convenience. According to the convention in (4.33), the homogeneous line bundle $\mathfrak{L}(\alpha)$ is then given by a quotient of $G \times \mathbb{C}$ under the action of $T=U(1)^{r}$ as

$$
t \cdot(g, v)=\left(g t^{-1}, \varrho_{\alpha}(t) \cdot v\right), \quad g \in G, \quad v \in \mathbb{C}, \quad t \in T,
$$

where

$$
\varrho_{\alpha}(t)=\exp [\mathrm{i}\langle\alpha, \xi\rangle], \quad t=\exp (\xi), \quad \xi \in \mathfrak{t} .
$$

In these terms we identify $\mathcal{O}_{\lambda}=G / T$, with $U_{0}=g \lambda g^{-1}$. Without loss we set

$$
\lambda=i \operatorname{diag}\left(\lambda_{1}, \ldots, \lambda_{r+1}\right), \quad \lambda_{1}+\cdots+\lambda_{r+1}=0,
$$

where regularity of $\lambda>0$ implies

$$
\lambda_{1}>\lambda_{2}>\cdots>\lambda_{r+1}
$$

At the identity in $G$, the eigenspaces $\left\{L_{1}, \ldots, L_{r+1}\right\}$ appearing in the decomposition (5.48) of $\left.V_{y}\right|_{p} \cong \mathbb{C}^{r+1}$ are spanned by the standard basis vectors $\left\{e_{1}, \ldots, e_{r+1}\right\}$, upon which $U_{0}=\lambda$ acts as $U_{0} \cdot e_{j}=\lambda_{j} e_{j}$ for $j=1, \ldots$, $r+1$. Explicitly, $e_{1}=(1,0, \ldots, 0), e_{2}=(0,1, \ldots, 0)$, and so on. More generally, if $U_{0}=g \lambda g^{-1}$, then each eigenspace $L_{j}$ is the subspace of $\mathbb{C}^{r+1}$ spanned by $g \cdot e_{j}$, where $g \in S U(r+1)$ acts on $e_{j}$ by ordinary matrix multiplication. Therefore, under the action $g \mapsto g t^{-1}$ as in (5.50), elements in $L_{j}$ are multiplied by the phase

$$
\varrho_{j}(t)=\exp \left(-\mathrm{i} \xi_{j}\right), \quad t=\operatorname{diag}\left(\mathrm{e}^{i \xi_{1}}, \ldots, \mathrm{e}^{i \xi_{r+1}}\right)
$$

To specify the associated line bundle on $\mathcal{O}_{\lambda}$, let us introduce a standard set of generators for the weight lattice $\Gamma_{\mathrm{wt}}$ of $S U(r+1)$. If $\xi=i \operatorname{diag}\left(\xi_{1}, \ldots\right.$, $\left.\xi_{r+1}\right)$ is any element in the Cartan subalgebra of $S U(r+1)$, we define weights $\left\{\hat{\omega}_{1}, \ldots, \hat{\omega}_{r+1}\right\}$ such that

$$
\begin{aligned}
& \left\langle\hat{\omega}_{j}, \xi\right\rangle=\xi_{j}, \quad j=1, \ldots, r+1, \\
& \xi=\operatorname{idiag}\left(\xi_{1}, \ldots, \xi_{r+1}\right), \quad \xi_{1}+\cdots+\xi_{r+1}=0 .
\end{aligned}
$$


"ATMP-17-1-A1-BEA" — 2013/5/15 — 19:32 — page 93 — \#93

This generating set of weights is slightly redundant, since

$$
\hat{\omega}_{1}+\cdots+\hat{\omega}_{r+1}=0
$$

but these generators also have the virtue of being simply permuted by the Weyl group of $S U(r+1)$. As usual, in terms of $\left\{\hat{\omega}_{1}, \ldots, \hat{\omega}_{r+1}\right\}$, the positive roots of $S U(r+1)$ are given by the successive differences $\hat{\omega}_{j}-\hat{\omega}_{\ell}$ for all pairs $j<\ell$.

Clearly from (5.54) and (5.55),

$$
\varrho_{j}(t)=\exp \left[-\mathrm{i}\left\langle\hat{\omega}_{j}, \xi\right\rangle\right]
$$

So according to the definition in (5.50), the eigenspace $L_{j}$ transforms as the fiber of the homogeneous line bundle $\mathfrak{L}\left(-\hat{\omega}_{j}\right)$ over $\mathcal{O}_{\lambda}$, and the weights $\alpha_{j}$ appearing in (5.49) are precisely the fundamental weights of $S U(r+1)$,

$$
\left.\mathcal{L}_{j}\right|_{\mathcal{O}_{\lambda}}=\mathfrak{L}\left(-\hat{\omega}_{j}\right), \quad j=1, \ldots, r+1 .
$$

As a special case, for $G=S U(2)$ and $\mathcal{O}_{\lambda} \cong \mathbb{C P}^{1}$, the minus sign in (5.58) is consistent with the standard identification of the tautological line bundle on $\mathbb{C P}^{1}$ as the line bundle of degree -1 .

Since $\mathcal{L}_{j}$ restricts fiberwise on $\mathcal{N}(P ; \lambda)$ to the the homogeneous line bundle $\mathfrak{L}\left(-\hat{\omega}_{j}\right)$, the Chern root $\mathfrak{u}_{j}$ in $(5.45)$ likewise restricts fiberwise to the Chern class of $\mathfrak{L}\left(-\hat{\omega}_{j}\right)$. But as we reviewed in the context of geometric quantization in Section 4.1, the Chern class of $\mathfrak{L}\left(-\hat{\omega}_{j}\right)$ is represented by the coadjoint form $-\nu_{\hat{\omega}_{j}} / 2 \pi$. The Chern root $\mathfrak{u}_{j}$ is therefore represented fiberwise on $\mathcal{N}(P ; \lambda)$ by

$$
\left.\mathrm{u}_{j}\right|_{\mathcal{O}_{\lambda}}=c_{1}\left(\mathfrak{L}\left(-\hat{\omega}_{j}\right)\right)=-\frac{\nu_{\hat{\omega}_{j}}}{2 \pi}, \quad j=1, \ldots, r+1 .
$$

In (5.59), we divide the curvature of the invariant connection on $\mathfrak{L}\left(-\hat{\omega}_{j}\right)$ by $2 \pi$ to ensure that $\mathfrak{u}_{j}$ has integral periods.

The formula in (5.59) has an important consequence. As before, the weights $\left\{\hat{\omega}_{1}, \ldots, \hat{\omega}_{r}\right\}$ provide a basis for the weight lattice $\Gamma_{\mathrm{wt}}$ of $S U(r+1)$. Under the isomorphism $\Gamma_{\mathrm{wt}} \cong H^{2}(G / T ; \mathbb{Z})$ in (4.26), the corresponding forms $\left\{\nu_{\hat{\omega}_{1}} / 2 \pi, \ldots, \nu_{\hat{\omega}_{r}} / 2 \pi\right\}$ then span $H^{2}(G / T ; \mathbb{Z})$. So via (5.59), the fiberwise component of any class in $H^{2}(\mathcal{N}(P ; \lambda) ; \mathbb{Z})$ can be expanded in terms of the Chern roots $\mathfrak{u}_{j}$ of the universal bundle $\mathcal{V}_{p}$.

Let us apply this observation to the two-form $e_{\lambda}$, which is the fiberwise component of the symplectic form $\Omega_{\lambda}$ on $\mathcal{N}(P ; \lambda)$. With $\lambda=i \operatorname{diag}\left(\lambda_{1}, \cdots\right.$, 
$\left.\lambda_{r+1}\right)$ as before, $\lambda$ can be expanded in terms of the weights $\hat{\omega}_{j} \operatorname{as}^{21}$

$$
\lambda=\lambda_{1} \hat{\omega}_{1}+\cdots+\lambda_{r+1} \hat{\omega}_{r+1}
$$

Being careful about the prefactor in (5.59), we thence obtain

$$
e_{\lambda}=-\sum_{j=1}^{r+1} 2 \pi \lambda_{j} u_{j}
$$

Equivalently, we regard $u=i \operatorname{diag}\left(\mathfrak{u}_{1}, \ldots, \mathfrak{u}_{r+1}\right)$ as a two-form on $\mathcal{N}(P ; \lambda)$ taking values in the Cartan subalgebra $\mathfrak{t}$ of $S U(r+1)$,

$$
\mathfrak{u}=i \operatorname{diag}\left(\mathfrak{u}_{1}, \ldots, \mathfrak{u}_{r+1}\right) \in H^{2}(\mathcal{N}(P ; \lambda) ; \mathbb{Z}) \otimes \mathfrak{t}
$$

such that

$$
\mathfrak{u}_{j}=\left\langle\hat{\omega}_{j}, \mathfrak{u}\right\rangle, \quad j=1, \ldots, r+1 .
$$

So more invariantly, the description of $e_{\lambda}$ in (5.61) becomes

$$
e_{\lambda}=-2 \pi(\lambda, u)
$$

Together, (5.32) and (5.64) finally imply that the symplectic form $\Omega_{\lambda}$ on $\mathcal{N}(P ; \lambda)$ is given by

$$
\Omega_{\lambda}=\mathrm{q}^{*} \Omega+4 \pi^{2}(\lambda, \mathrm{u})
$$

In Section 7.3, we will make essential use of this expression relating $\Omega_{\lambda}$ to the symplectic form $\Omega$ on $\mathcal{N}(P)$ and the Chern roots $u$ of $\mathcal{V}_{p}$.

\section{General aspects of non-abelian localization}

In preparation for the computations in Section 7, we quickly review in this section some general aspects of non-abelian localization. Our goals are to recall the philosophy which underlies computations via non-abelian localization and to state the basic localization formula derived in [18]. Since the latter result may be useful elsewhere, I have decided to present it in somewhat greater generality than we will actually require for our study of the Seifert loop operator.

\footnotetext{
${ }^{21}$ Again, we use the invariant metric given by $-\operatorname{Tr}$ to identify $\mathfrak{t} \cong \mathfrak{t}^{*}$.
} 
"ATMP-17-1-A1-BEA" — 2013/5/15 — 19:32 — page 95 — \#95

Very broadly, non-abelian localization provides a general means to study a symplectic integral of the canonical form

$$
Z(\epsilon)=\frac{1}{\operatorname{Vol}(H)}\left(\frac{1}{2 \pi \epsilon}\right)^{\Delta_{H} / 2} \int_{X} \exp \left[\Omega-\frac{1}{2 \epsilon}(\mu, \mu)\right], \quad \Delta_{H}=\operatorname{dim} H
$$

Here, $X$ is a symplectic manifold with symplectic form $\Omega$, and $H$ is a Lie group, which acts on $X$ in a Hamiltonian fashion with moment map $\mu$. Finally, $(\cdot, \cdot)$ is an invariant, positive-definite ${ }^{22}$ quadratic form on the Lie algebra $\mathfrak{h}$ of $H$ and dually on $\mathfrak{h}^{*}$, which we use to define the "action" $S=$ $\frac{1}{2}(\mu, \mu)$ and the volume $\operatorname{Vol}(H)$ of $H$ that appear in (6.1).

To apply non-abelian localization to an integral of the form (6.1), we first observe that $Z(\epsilon)$ can be rewritten as

$$
Z(\epsilon)=\frac{1}{\operatorname{Vol}(H)} \int_{\mathfrak{h} \times X}\left[\frac{d \phi}{2 \pi}\right] \exp \left[\Omega-\mathrm{i}\langle\mu, \phi\rangle-\frac{\epsilon}{2}(\phi, \phi)\right]
$$

Here, $\phi$ is an element of the Lie algebra $\mathfrak{h}$ of $H$, and $[d \phi]$ is the Euclidean measure on $\mathfrak{h}$ that is determined by the same invariant form $(\cdot, \cdot)$, which we use to define the volume $\operatorname{Vol}(H)$ of $H$. To avoid confusion, the measure $[d \phi / 2 \pi]$ includes a factor of $1 / 2 \pi$ for each real component of $\phi$. The Gaussian integral over $\phi$ in (6.2) then leads immediately to the expression for $Z$ in (6.1).

\subsection{BRST symmetry}

The great advantage of writing $Z$ in the form (6.2) is that, once we introduce $\phi$, the integrand of $Z$ becomes invariant under a BRST symmetry, which leads directly to a localization result for (6.1). Specifically, as first demonstrated by Witten in [108], the canonical symplectic integral in (6.1) can be evaluated as a sum of local contributions from the neighborhoods of the critical points of the function $S=\frac{1}{2}(\mu, \mu)$.

\footnotetext{
${ }^{22}$ In the case of Chern-Simons theory, the corresponding quadratic form (3.38) on $\mathfrak{h}$ has indefinite signature, due to the hyperbolic summand associated to the two extra $U(1)$ generators of $\mathcal{H}=U(1)_{\mathrm{R}} \ltimes \widetilde{\mathcal{G}}_{0}$ relative to the group of gauge transformations $\mathcal{G}_{0}$. As a related fact, invariance under large gauge transformations requires the Chern-Simons symplectic integral (3.49) to be oscillatory, instead of exponentially damped. These features do not essentially change our general discussion of localization.
} 
To describe the BRST symmetry of (6.2), we recall that the moment map satisfies

$$
d\langle\mu, \phi\rangle=\iota_{V(\phi)} \Omega,
$$

where $V(\phi)$ is the vector field on $X$ associated to the infinitesimal action of $\phi$. Because of the relation (6.3), the argument of the exponential in (6.2) is immediately annihilated by the BRST operator $D$ defined by

$$
D=d+\mathrm{i} \iota_{V(\phi)} .
$$

To exhibit the action of $D$ locally, we choose a basis $\phi^{a}$ for $\mathfrak{h}$, and we introduce local coordinates $x^{m}$ on $X$. We also introduce the fermionic notation $\chi^{m} \equiv d x^{m}$ for the corresponding basis of local one-forms on $X$, and we expand the vector field $V(\phi)$ into components as $V(\phi)=\phi^{a} V_{a}^{m} \partial / \partial x^{m}$. Then the action of $D$ in (6.4) is described in terms of these local coordinates by

$$
\begin{aligned}
D x^{m} & =\chi^{m}, \\
D \chi^{m} & =\mathrm{i} \phi^{a} V_{a}^{m}, \\
D \phi^{a} & =0 .
\end{aligned}
$$

From this local description (6.5), we see that the action of $D$ preserves a ghost number, or grading, under which $x$ carries charge $0, \chi$ carries charge $+1, \phi$ carries charge +2 , and $D$ itself carries charge +1 .

The most important property of a BRST operator is that it squares to zero. In this case, either from (6.4) or from (6.5), we see that $D$ squares to the Lie derivative along the vector field $V(\phi)$,

$$
D^{2}=i\left\{d, \iota_{V(\phi)}\right\}=\mathrm{i} £_{V(\phi)} .
$$

Thus, $D^{2}=0$ exactly when $D$ acts on the subspace of $H$-invariant functions $\mathcal{O}(x, \chi, \phi)$ of $x, \chi$, and $\phi$.

For simplicity, we restrict attention to functions $\mathcal{O}(x, \chi, \phi)$, which are polynomial in $\phi$. Then an arbitrary function of this form can be expanded as a sum of terms

$$
\mathcal{O}(x)_{m_{1} \ldots m_{p} a_{1} \ldots a_{q}} \chi^{m_{1}} \cdots \chi^{m_{p}} \phi^{a_{1}} \cdots \phi^{a_{q}},
$$

for some $0 \leq p \leq \operatorname{dim} X$ and $q \geq 0$. (The restriction on $p$ arises from the fact that $\chi$ satisfies Fermi statistics, whereas $\phi$ satisfies Bose statistics.) 
Globally, each term of the form (6.7) is specified by a section of the bundle $\Omega_{X}^{p} \otimes \operatorname{Sym}^{q}\left(\mathfrak{h}^{*}\right)$ of $p$-forms on $X$ taking values in the $q$-th symmetric tensor product of the dual $\mathfrak{h}^{*}$ of the Lie algebra of $H$. Thus, if we consider the complex $\left(\Omega_{X}^{*} \otimes \operatorname{Sym}^{*}\left(\mathfrak{h}^{*}\right)\right)^{H}$ of all $H$-invariant differential forms on $X$ which take values in the ring of polynomial functions on $\mathfrak{h}$, then we see that $D$ defines a cohomology theory associated to the action of $H$ on $X$. This cohomology theory is known as the Cartan model for the $H$-equivariant cohomology $H_{H}^{*}(X)$ of $X$. See $[11,56]$ for nice references on equivariant cohomology in the spirit of the present paper.

For later discussion we require a few elementary properties of equivariant cohomology; let us record them now. First, in the special case that $X$ is a point, the $H$-equivariant cohomology ring of $X$ is simply the ring of invariant functions on $\mathfrak{h}$. That is,

$$
H_{H}^{*}(p t)=\operatorname{Sym}^{*}\left(\mathfrak{h}^{*}\right)^{H} .
$$

Similarly, if $X$ is not necessarily a point but $H$ still acts trivially on $X$, the $H$-equivariant cohomology ring of $X$ is the tensor product of the ordinary cohomology ring of $X$ and the ring of invariant functions on $\mathfrak{h}$, so that

$$
H_{H}^{*}(X)=H^{*}(X) \otimes H_{H}^{*}(p t) .
$$

Both of these statements follow immediately from our definition of the Cartan model. Finally, at the opposite extreme that $H$ acts freely on $X$, the $H$-equivariant cohomology of $X$ is isomorphic to the ordinary cohomology of the quotient $X / H$,

$$
H_{H}^{*}(X)=H^{*}(X / H) .
$$

This exceedingly natural statement is less obvious in the Cartan model, but it is often taken as the starting point in other topological models for equivariant cohomology, as explained in Ch. 1 of [56].

\subsubsection{Localization for $Z$}

Since the argument of the exponential in (6.2) is annihilated by $D$ and because this argument is manifestly invariant under $H$, the integrand of the canonical symplectic integral determines an equivariant cohomology class on $X$. Furthermore, by the usual arguments, $Z$ in (6.2) is formally unchanged under the addition of any $D$-exact invariant form to its integrand. This formal statement can fail if $X$ is not compact and $Z$ suffers from divergences. To avoid such complications, we assume that $X$ is compact but see Appendix A of [18] for an example of the sort of analysis required 
when this assumption is relaxed. Thus, $Z$ depends only on the equivariant cohomology class of its integrand.

We now explain how this observation leads immediately to a localization result for $Z$. We first note that we can add to the argument of the exponential in (6.2) an arbitrary term of the form $s D \Psi$, where $\Psi$ is any $H$-invariant one-form on $X$ and $s$ is a real parameter. As a result,

$$
Z(\epsilon)=\frac{1}{\operatorname{Vol}(H)} \int_{\mathfrak{h} \times X}\left[\frac{d \phi}{2 \pi}\right] \exp \left[\Omega-i\langle\mu, \phi\rangle-\frac{\epsilon}{2}(\phi, \phi)+s D \Psi\right] .
$$

This deformation of the integrand of (6.2) is $D$-exact and does not change $Z$. In particular, $Z$ does not depend on $s$.

By definition, since $\Psi$ is a one-form, $D \Psi$ is given explicitly by

$$
D \Psi=d \Psi+\mathrm{i}\langle\Psi, V(\phi)\rangle .
$$

As before, $\langle\cdot, \cdot\rangle$ denotes the canonical dual pairing, so that in components the last term of (6.12) is given by i $\Psi_{m} V_{a}^{m} \phi^{a}$. Thus, apart from a polynomial in $s$ that arises from expanding the exponential term $\exp (s d \Psi)$ in (6.11), all of the dependence on $s$ in the integrand of $Z$ arises from the factor $\exp [\mathrm{i} s\langle\Psi, V(\phi)\rangle]$ that now appears in (6.11). So if we consider the limit $s \rightarrow \infty$, then the stationary phase approximation to the integral is valid, and all contributions to $Z$ localize around the critical points of the function $\langle\Psi, V(\phi)\rangle$.

To determine the critical points of $\langle\Psi, V(\phi)\rangle$, we expand this function in the basis $\phi^{a}$ for $\mathfrak{h}$ which we introduced previously,

$$
\langle\Psi, V(\phi)\rangle=\phi^{a}\left\langle\Psi, V_{a}\right\rangle .
$$

Thus, the critical points of $\langle\Psi, V(\phi)\rangle$ arise from the simultaneous solutions in $\mathfrak{h} \times X$ of the equations

$$
\begin{aligned}
\left\langle\Psi, V_{a}\right\rangle & =0, \\
\phi^{a} d\left\langle\Psi, V_{a}\right\rangle & =0 .
\end{aligned}
$$

The first equation in (6.14) implies that $Z$ necessarily localizes on points in $\mathfrak{h} \times X$ for which $\left\langle\Psi, V_{a}\right\rangle$ vanishes. As for the second equation in (6.14), we see that it is invariant under an overall scaling of $\phi$ in the vector space $\mathfrak{h}$. Consequently, upon integrating over $\phi$ in (6.11), we see that the critical locus of the function $\langle\Psi, V(\phi)\rangle$ in $\mathfrak{h} \times X$ projects onto the vanishing locus of $\left\langle\Psi, V_{a}\right\rangle$ in $X$. So $Z$ localizes on the subset of $X$ where $\left\langle\Psi, V_{a}\right\rangle=0$. 
"ATMP-17-1-A1-BEA" — 2013/5/15 — 19:32 — page 99 — \#99

LOCALIZATION FOR WILSON LOOPS IN CHERN-SIMONS

By making a specific choice of the one-form $\Psi$, we can describe the localization of $Z$ more precisely. In particular, we now show that $Z$ localizes on the set of critical points of the invariant function $S=\frac{1}{2}(\mu, \mu)$ on $X$.

We begin by choosing an almost complex structure $\mathbf{J}$ on $X$. That is, $\mathbf{J}: T X \rightarrow T X$ is a linear map from $T X$ to itself such that $\mathbf{J}^{2}=-1$. We assume that $\mathbf{J}$ is compatible with the symplectic form $\Omega$ in the sense that $\Omega$ is of type $(1,1)$ with respect to $\mathbf{J}$ and the associated metric $(\cdot, \cdot) \equiv \Omega(\cdot, \mathbf{J} \cdot)$ on $X$ is positive-definite. Such an almost complex structure always exists.

Using $\mathbf{J}$ and $S$, we now introduce the invariant one-form

$$
\Psi=\mathbf{J} d S=(\mu, \mathbf{J} d \mu)
$$

In components, $\Psi=d x^{m} \mathbf{J}_{m}^{n} \partial_{n} S=d x^{m} \mu^{a} \mathbf{J}_{m}^{n} \partial_{n} \mu_{a}$.

The integral $Z$ now localizes on the subset of $X$ where $\left\langle\Psi, V_{a}\right\rangle=0$. Comparing to (6.15), we see that this subset certainly includes all critical points of $S$, since by definition $d S=0$ at these points. Conversely, we now show that if $\left\langle\Psi, V_{a}\right\rangle=0$ at some point on $X$, then this point is a critical point of $S$. To prove this assertion, we use the inverse $\Omega^{-1}$ to $\Omega$, which arises by considering the symplectic form as an isomorphism $\Omega: T M \rightarrow T^{*} M$ with inverse $\Omega^{-1}: T^{*} M \rightarrow T M$. In components, $\Omega^{-1}$ is defined by $\left(\Omega^{-1}\right)^{l m} \Omega_{m n}=\delta_{n}^{l}$.

In terms of $\Omega^{-1}$, the moment map equation (6.3) is equivalent to the relation

$$
V=\Omega^{-1} d \mu
$$

or $V_{a}^{m}=\left(\Omega^{-1}\right)^{m n} \partial_{n} \mu_{a}$. Thus,

$$
\Omega^{-1} d S=\left(\mu, \Omega^{-1} d \mu\right)=(\mu, V),
$$

or $\left(\Omega^{-1}\right)^{m n} \partial_{n} S=\mu^{a} V_{a}^{m}$.

In particular, the condition that $\left\langle\Psi, V_{a}\right\rangle=0$ implies that

$$
0=(\mu,\langle\Psi, V\rangle)=\left\langle\Psi, \Omega^{-1} d S\right\rangle=\left\langle\mathbf{J} d S, \Omega^{-1} d S\right\rangle,
$$

or more explicitly, $0=\mu^{a} \Psi_{m} V_{a}^{m}=\Psi_{m}\left(\Omega^{-1}\right)^{m n} \partial_{n} S=\left(\Omega^{-1}\right)^{m n} \mathbf{J}_{m}^{l} \partial_{l} S \partial_{n} S$. We recognize the last expression in (6.18) as the norm of the one-form $d S$ with respect to the metric on $X$. Because this metric is positive-definite, we conclude that the condition $\left\langle\Psi, V_{a}\right\rangle=0$ implies the vanishing of $d S$. Thus the symplectic integral $Z$ localizes exactly on the critical set of $S=\frac{1}{2}(\mu, \mu)$. 
"ATMP-17-1-A1-BEA" — 2013/5/15 — 19:32 — page 100 — \#100

\subsection{A non-abelian localization formula}

In order to pass from the preceding general statement to a precise localization formula for $Z$, we must introduce a model for the local symplectic geometry near a critical point of $S$. In this context, one of the important technical results of [18] was to identify a "canonical" symplectic model appropriate to describe the local geometry in $\mathcal{A}(P)$ near a critical point of the two-dimensional Yang-Mills action, or alternatively, to describe the local geometry in $\overline{\mathcal{A}}$ near a critical point of the shift-invariant Chern-Simons action. Happily, the canonical symplectic model deserves its name, insofar as it suffices also to describe the local symplectic geometry in the loopspace $L \mathcal{O}_{\alpha}$ relevant for our computations of the Seifert loop path integral.

To keep the paper to a reasonable length, we will present the canonical symplectic model and state the attendant localization formula for $Z$ more or less as a fait accompli. See Sections 4.3 and 5.3 of [18] for a detailed explanation of how this model arises from two-dimensional Yang-Mills theory, along with a careful derivation of the non-abelian localization formula. We refer the reader to the work [92] of Paradan for a related general analysis of non-abelian localization. Specific applications of non-abelian localization to gauge theory have also been discussed by Teleman and Woodward in $[100,101,110]$ and by Blau and Thompson in $[20,22]$.

\subsubsection{The canonical symplectic model in brief}

Before we can even state the non-abelian localization formula, we need to establish a model for the local symplectic geometry to which it applies. Somewhat abstractly, let us consider a connected component $\mathcal{C}$ in the critical locus of the function $S=\frac{1}{2}(\mu, \mu)$ on $X$. Since $S$ is invariant under the connected Hamiltonian group $H$, the action of $H$ on $X$ automatically preserves $\mathcal{C}$. The following local model for a symplectic neighborhood of $\mathcal{C}$ in $X$ is certainly not the most general, and in particular it relies on two simplifying assumptions about the way $H$ acts on $\mathcal{C}$. Roughly speaking, both of these assumptions mean that $H$ acts uniformly on $\mathcal{C}$.

First, we assume that the stabilizer of each point in $\mathcal{C}$ is isomorphic to a fixed subgroup $H_{0} \subseteq H$. Of course, we allow the possibility that $H$ acts freely near $\mathcal{C}$, so that $H_{0}$ is trivial. Because all points of $\mathcal{C}$ have the same stabilizer, which is certainly a restrictive condition in general, the quotient $\mathcal{M}=\mathcal{C} / H$ is smooth. (But see work [66] of Jeffrey and collaborators for a very interesting example of non-abelian localization in a case where $\mathcal{M}$ is singular.) 
"ATMP-17-1-A1-BEA" — 2013/5/15 — 19:32 — page 101 — \#101

Second, we assume that the moment map $\mu$ on $\mathcal{C}$ takes values in a single, fixed coadjoint orbit in $\mathfrak{h}^{*}$. Somewhat perversely, we again dually parameterize the value of the moment map on $\mathcal{C}$ by an element $\gamma_{0}$ in $\mathfrak{h}$, the Lie algebra of $H$.

By the assumptions so far, $\gamma_{0}$ cannot be an arbitrary element of $\mathfrak{h}$ but must be a central element in the Lie algebra $\mathfrak{h}_{0}$ of the stabilizer group $H_{0}$. In identifying $\gamma_{0}$ as an element of $\mathfrak{h}_{0}$, we note that at a point $x_{0}$ in $\mathcal{C}$ for which $d S=(\mu, d \mu)=0$, the moment map $\mu\left(x_{0}\right)$ satisfies $\left(\mu\left(x_{0}\right), V\right)=$ $\mu\left(x_{0}\right)^{a} V_{a}^{m}=0$ via (6.16) and (6.17). Since $\gamma_{0}$ is the dual of $\mu\left(x_{0}\right)$, the vector field generated by the action of $\gamma_{0}$ vanishes at $x_{0}$, and $\gamma_{0}$ therefore lies in $\mathfrak{h}_{0}$. As $H_{0}$ fixes $x_{0}$, the Hamiltonian condition on the moment map $\mu$ at $x_{0}$ further implies

$$
H_{0} \gamma_{0} H_{0}^{-1}=\gamma_{0}
$$

Thus, $\gamma_{0}$ is central in $\mathfrak{h}_{0}$. As a small consistency check, we note that the restriction $\left.S\right|_{\mathcal{C}}=\frac{1}{2}\left(\gamma_{0}, \gamma_{0}\right)$ is automatically constant on $\mathcal{C}$ by the second assumption above. Of course, if $\mathcal{C}$ is a component of the critical locus of $S$, then $S$ is necessarily constant on $\mathcal{C}$.

We now describe an $H$-invariant neighborhood $N$ of $\mathcal{C} \subset X$ as the total space of an $H$-equivariant bundle with fiber $F$ over the quotient $\mathcal{M}=\mathcal{C} / H$,

$$
F \longrightarrow \underset{\mathcal{M}}{\stackrel{\downarrow}{\downarrow} \operatorname{pr}}, \quad \mathcal{M}=\mathcal{C} / H .
$$

In these terms, our canonical symplectic model amounts to a description of the fiber $F$ in (6.20) as a symplectic manifold equipped with a Hamiltonian action of $H$. Since we assume $F$ to be symplectic (and the fibration over $\mathcal{M}$ to respect that symplectic structure), the symplectic form on $N$ immediately induces a corresponding symplectic form on the base $\mathcal{M}$ of (6.20).

Let us first recall the topological model for $F$. Since $H$ acts on each point in $\mathcal{C}$ with stabilizer $H_{0}, F$ contains a copy of the homogeneous space $H / H_{0}$. To incorporate the normal directions to $\mathcal{C}$ in $F$ as well, we take $F$ to be a homogeneous vector bundle over $H / H_{0}$ of the form

$$
F=H \times_{H_{0}}\left(\mathfrak{h}^{\perp} \oplus E_{1}\right), \quad \mathfrak{h}^{\perp} \equiv \mathfrak{h} \ominus \mathfrak{h}_{0} \ominus E_{0} .
$$

The description of $F$ in (6.21) requires a bit of explanation. Besides the data of $H_{0}$ and $\gamma_{0}$, the canonical symplectic model for $F$ is parameterized by the choice of vector spaces $E_{0}$ and $E_{1}$. More precisely, $E_{0}$ is a subspace of $\mathfrak{h}$ 
"ATMP-17-1-A1-BEA" — 2013/5/15 — 19:32 — page 102 — \#102

which has trivial intersection with $\mathfrak{h}_{0}$ and is preserved under the adjoint action of $H_{0}$, so that infinitesimally $\left[\mathfrak{h}_{0}, E_{0}\right] \subseteq E_{0}$. Given the invariant metric $(\cdot, \cdot)$ on $\mathfrak{h}$, we then define $\mathfrak{h}^{\perp}=\mathfrak{h} \ominus \mathfrak{h}_{0} \ominus E_{0}$ as the orthocomplement to the direct sum $\mathfrak{h}_{0} \oplus E_{0}$ in $\mathfrak{h}$. Similarly, $E_{1}$ is a vector space on which $H_{0}$ acts in some representation (not necessarily the adjoint), and we assume that $E_{1}$ also carries a Euclidean metric invariant under the action of $H_{0}$.

Throughout the following, we use $h$ to denote an element of $H, \gamma$ to denote an element of $\mathfrak{h}^{\perp}$, and $v$ to denote an element of $E_{1}$. With this notation, we define $F$ in (6.21) to be the quotient of the product $H \times\left(\mathfrak{h}^{\perp} \oplus E_{1}\right)$ under the action $^{23}$ of $H_{0}$ as

$$
h_{0} \cdot(h, \gamma, v)=\left(h h_{0}^{-1}, h_{0} \gamma h_{0}^{-1}, h_{0} \cdot v\right), \quad h_{0} \in H_{0} .
$$

To specify completely the local model, we must also discuss the symplectic structure and the Hamiltonian $H$-action on $F$. I will be fairly succinct here, since this material is covered in detail in Section 4.3 of [18] and is based upon standard techniques for constructing symplectic bundles, as explained for instance in Ch. 35-41 of [55].

In order to place a symplectic structure on $F$, we must make an additional assumption about the representations $E_{0}$ and $E_{1}$ of $H_{0}$. Namely, we assume that $\gamma_{0} \in \mathfrak{h}_{0}$ acts non-degenerately on both $E_{0}$ and $E_{1}$, with non-zero eigenvalues. Because the action of $\gamma_{0}$ preserves the invariant metrics on both $E_{0}$ and $E_{1}$, the action of $\gamma_{0}$ on each of these vector spaces is represented by a real, anti-symmetric matrix. The positive and negative eigenspaces of the Hermitian operator $-\mathrm{i} \gamma_{0}$ thereby determine complex structures on $E_{0}$ and $E_{1}$. Each of these complex structures is automatically preserved by $H_{0}$, and the invariant metrics on $E_{0}$ and $E_{1}$ are Hermitian.

The complex structures on $E_{0}$ and $E_{1}$ will be essential when we present the non-abelian localization formula, so let us describe them more precisely. By convention, we take elements of holomorphic/anti-holomorphic type $(1,0)$ in $E_{0}$ to lie in the positive eigenspace of $-i \gamma_{0}$, and we take corresponding elements of type $(1,0)$ in $E_{1}$ to lie in the negative eigenspace of $-\mathrm{i} \gamma_{0}$. That is, when acting on a standard holomorphic basis,

$$
-\mathrm{i} \gamma_{0}>0 \text { on } E_{0}, \quad-\mathrm{i} \gamma_{0}<0 \text { on } E_{1} \text {. }
$$

With this convention for the respective complex structures on $E_{0}$ and $E_{1}$, extraneous signs are suppressed in the non-abelian localization formula.

\footnotetext{
${ }^{23}$ Our convention that $H_{0}$ acts on $H$ from the right is opposite to the convention in [18] but agrees with the convention for coadjoint orbits in Section 4. As a result, some of the expressions in this section differ by signs from those in [18].
} 
"ATMP-17-1-A1-BEA" — 2013/5/15 - 19:32 — page 103 — \#103

Later, in Section 6.3, we illustrate the convention (6.23) in several elementary examples.

Without further ado, the symplectic form $\Omega$ on $F$ is given by

$$
\Omega=-d\left(\gamma+\gamma_{0}, \theta\right)-d\left\langle\mu_{E_{1}}, \theta\right\rangle+\Omega_{E_{1}}, \quad \theta=h^{-1} d h .
$$

Here $\Omega_{E_{1}}$ is the invariant symplectic form on $E_{1}$ determined by the aforementioned metric and complex structure. Also, $\mu_{E_{1}}$ is the moment map for the Hamiltonian action of $H_{0}$ on $E_{1}$. As we shall use in a moment, because the action of $H_{0}$ on $E_{1}$ is linear, the moment map $\mu_{E_{1}}$ is a homogeneous quadratic function of the vector $v \in E_{1}$.

The two-form $\Omega$ in (6.24) is manifestly closed and invariant under $H_{0}$. Further, if $V(\psi)$ denotes the vector field on $H \times\left(\mathfrak{h}^{\perp} \oplus E_{1}\right)$ generated by an element $\psi \in \mathfrak{h}_{0}$, then $\Omega$ vanishes upon contraction with $V(\psi)$. This statement can be checked directly from (6.24), once we note that $V(\psi)$ is given explicitly by

$$
\delta h=-h \cdot \psi, \quad \delta \gamma=[\psi, \gamma], \quad \delta v=\psi \cdot v .
$$

Under (6.25), the terms involving $\gamma$ and $\gamma_{0}$ in $\Omega$ are separately annihilated when contracted with $V(\psi)$, and we have carefully arranged the terms involving $\mu_{E_{1}}$ and $\Omega_{E_{1}}$ to cancel when contracted with $V(\psi)$. Thus $\Omega$ descends to a closed two-form on $F$.

We are left to check that $\Omega$ is non-degenerate as a symplectic form on a neighborhood of $H / H_{0}$ in $F$, where we embed $H / H_{0}$ as the base of the homogeneous bundle (6.21) at $\gamma=v=0$. Restricting $\Omega$ to $H / H_{0}$, thereby setting $\gamma=v=0$ in (6.24), we immediately obtain

$$
\left.\Omega\right|_{H / H_{0}}=-(d \gamma, \theta)-\left(\gamma_{0}, d \theta\right)+\Omega_{E_{1}} .
$$

Here we use that $\mu_{E_{1}}$, being quadratic in $v$, satisfies $\mu_{E_{1}}=d \mu_{E_{1}}=0$ at $v=0$, so that no terms involving $\mu_{E_{1}}$ appear above. The term involving $d \gamma$ in (6.26) then provides a non-degenerate, cotangent pairing between the tangent directions to $H / H_{0}$, which lie in $\mathfrak{h}^{\perp}=\mathfrak{h} \ominus \mathfrak{h}_{0} \ominus E_{0}$ and the corresponding directions in the fiber over $H / H_{0}$. The term involving $\gamma_{0}$ similarly pairs the remaining tangent directions to $H / H_{0}$ in $E_{0}$ via the coadjoint symplectic form introduced in (4.15). Finally, $\Omega_{E_{1}}$ is non-degenerate on $E_{1}$ by definition. So $\Omega$ in (6.24) defines a symplectic form on a neighborhood of $H / H_{0}$ in $F$. 
As for the Hamiltonian symmetry, we assume that $H$ acts from the left on $H / H_{0}$, so that

$$
h^{\prime} \cdot(h, \gamma, v)=\left(h^{\prime} h, \gamma, v\right), \quad h^{\prime} \in H .
$$

The corresponding element $\phi$ in $\mathfrak{h}$ generates the vector field

$$
\delta h=\phi \cdot h, \quad \delta \gamma=0, \quad \delta v=0 .
$$

Since the one-form $\theta$ appearing in $\Omega$ is left-invariant, the symplectic form on $F$ is manifestly invariant under $H$. Finally, using (6.24) and (6.28), one can easily check that the action of $H$ on $F$ is Hamiltonian with moment map $\mu$ given by

$$
\langle\mu, \phi\rangle=\left(\gamma+\gamma_{0}, h^{-1} \phi h\right)+\left\langle\mu_{E_{1}}, h^{-1} \phi h\right\rangle .
$$

As a small check, we note that the value of $\mu$ in (6.29) on $H / H_{0} \subset F$ is given by $\gamma_{0}$, as we initially assumed, and $H / H_{0}$ sits on the critical locus of $S=\frac{1}{2}(\mu, \mu)$.

\subsubsection{An equivariant Euler class from $F$}

Having fixed a symplectic model for $F$ in (6.20), we can now present the basic localization formula for the canonical symplectic integral in (6.1). To start, we define the local contribution to $Z$ from the component $\mathcal{C} \subset X$ by the following symplectic integral over $N$,

$$
\left.Z(\epsilon)\right|_{\mathcal{M}}=\frac{1}{\operatorname{Vol}(H)} \int_{\mathfrak{h} \times N}\left[\frac{d \phi}{2 \pi}\right] \exp \left[\Omega-\mathrm{i}\langle\mu, \phi\rangle-\frac{\epsilon}{2}(\phi, \phi)+s D \Psi\right] .
$$

So long as $s$ is non-zero and $\Psi$ is given by (6.15), the integral (6.30) over the non-compact space $N$ is both convergent and independent of $s$, so that $\left.Z(\epsilon)\right|_{\mathcal{M}}$ is well-defined.

In principle, to obtain a localization formula, we simply integrate over $\left(\mathfrak{h} \ominus \mathfrak{h}_{0}\right) \times F$ in (6.30), thereby reducing $\left.Z(\epsilon)\right|_{\mathcal{M}}$ to an integral over the reduced space $\mathfrak{h}_{0} \times \mathcal{M}$. In practice, because the integral in (6.30) does not become Gaussian even in the limit $s \rightarrow \infty$, the analysis of (6.30) is somewhat involved and occupies a significant fraction of [18]. Rather than repeat that analysis here, I will simply summarize its result. 
"ATMP-17-1-A1-BEA" — 2013/5/15 - 19:32 — page $105-\# 105$

In a nutshell, the non-abelian localization formula of [18] states that $\left.Z(\epsilon)\right|_{\mathcal{M}}$ in (6.30) is given by the following integral over $\mathfrak{h}_{0} \times \mathcal{M}$,

$$
\begin{aligned}
\left.Z(\epsilon)\right|_{\mathcal{M}}= & \frac{1}{\operatorname{Vol}\left(H_{0}\right)} \int_{\mathfrak{h}_{0} \times \mathcal{M}}\left[\frac{d \psi}{2 \pi}\right] \frac{e_{H_{0}}\left(\mathcal{M}, E_{0}\right)}{e_{H_{0}}\left(\mathcal{M}, E_{1}\right)} \\
& \times \exp \left[\Omega+\epsilon \Theta-\mathrm{i}\left(\gamma_{0}, \psi\right)-\frac{\epsilon}{2}(\psi, \psi)\right] .
\end{aligned}
$$

This formula deserves a number of comments.

Let us first explain our notation. The integration variable $\psi$ in (6.31) is an element in $\mathfrak{h}_{0}$, and $\Omega$ and $\Theta$ are classes in the ordinary de Rham cohomology ring $H^{*}(\mathcal{M})$ of $\mathcal{M}$. As the notation suggests, $\Omega$ is the symplectic form on $\mathcal{M}$ inherited from $X$, and $\Theta$ is a degree-four characteristic class on $\mathcal{M}$ that we specify more precisely below. Finally, $e_{H_{0}}\left(\mathcal{M}, E_{0}\right)$ and $e_{H_{0}}\left(\mathcal{M}, E_{1}\right)$ are the $H_{0}$-equivariant Euler classes of complex vector bundles over $\mathcal{M}$ associated to $E_{0}$ and $E_{1}$. Again, we provide a more explicit description of $e_{H_{0}}\left(\mathcal{M}, E_{0}\right)$ and $e_{H_{0}}\left(\mathcal{M}, E_{1}\right)$ below. For now, we simply note that these classes are defined as elements in the $H_{0}$-equivariant cohomology ring $H_{H_{0}}^{*}(\mathcal{M})$ of $\mathcal{M}$.

In fact, all terms in the integrand of (6.31) can be understood as classes in $H_{H_{0}}^{*}(\mathcal{M})$. Because $H_{0}$ acts trivially on $\mathcal{M}$, the $H_{0}$-equivariant cohomology ring of $\mathcal{M}$ is given by the tensor product of the ordinary cohomology ring of $\mathcal{M}$ with the ring of invariant functions on $\mathfrak{h}_{0}$. Identifying the ring of invariant functions on $\mathfrak{h}_{0}$ with the $H_{0}$-equivariant cohomology of a point, we thus write

$$
H_{H_{0}}^{*}(\mathcal{M})=H^{*}(\mathcal{M}) \otimes H_{H_{0}}^{*}(p t) .
$$

Perhaps a bit more concretely, elements in $H_{H_{0}}^{*}(\mathcal{M})$ can be written as sums of terms having the form $x \cdot f(\psi)$, where $x$ is an ordinary cohomology class on $\mathcal{M}$ and $f(\psi)$ is an invariant function of $\psi$. Recalling that $\gamma_{0} \in \mathfrak{h}_{0}$ is invariant under $H_{0}$, we thus see that the terms appearing in the argument of the exponential in (6.31) can also be considered as classes in $H_{H_{0}}^{*}(\mathcal{M})$.

The appearance of $H_{H_{0}}^{*}(\mathcal{M})$ in the non-abelian localization formula is no accident. As we observed in Appendix $\mathrm{C}$ of [18], the pullback pr* from $\mathcal{M}$ to $N$ in (6.20) induces an isomorphism, at least rationally, between the $H_{0^{-}}$ equivariant cohomology ring of $\mathcal{M}$ and the $H$-equivariant cohomology ring of $N$. Under this isomorphism, the $H$-equivariant classes on $N$ represented by $[\Omega-\mathrm{i}\langle\mu, \phi\rangle]$ and $\left[-\frac{1}{2}(\phi, \phi)\right]$ in (6.30) are the pullbacks from corresponding $H_{0}$-equivariant classes on $\mathcal{M}$. 
To identify the relevant classes in $H_{H_{0}}^{*}(\mathcal{N})$, let us quickly recall how the identification between $H_{H}^{*}(N)$ and $H_{H_{0}}^{*}(\mathcal{M})$ formally arises. Given the symplectic model (6.21) for $F$ as an equivariant vector bundle over $H / H_{0}$, the neighborhood $N$ equivariantly retracts onto a bundle $\bar{N}$ with fibers $H / H_{0}$ over $\mathcal{M}$,

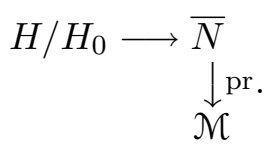

Under this retraction, we identify $H_{H}^{*}(N)=H_{H}^{*}(\bar{N})$.

For the moment, let us suppose that $\bar{N}$ can be constructed as a quotient of a principal $H$-bundle $P_{H}$ over $\mathcal{M}$. Thus $P_{H}$ is a bundle with fiber $H$ over $\mathcal{M}$. Besides the given action of $H$, we assume that $P_{H}$ admits a free action of $H_{0}$ commuting with the action of $H$, so that $\bar{N}=P_{H} / H_{0}$. On the other hand, if we take the quotient of $P_{H}$ by the free action of $H$, we identify $\mathcal{M}=P_{H} / H$. Considering these quotients in successive orders, we obtain the required isomorphism

$$
H_{H}^{*}(\bar{N})=H_{H \times H_{0}}^{*}\left(P_{H}\right)=H_{H_{0}}^{*}(\mathcal{M}) .
$$

Finally, although the requisite $P_{H}$ in (6.34) may or may not exist on the nose, $P_{H}$ always exists rationally, which suffices for our application to the Cartan model.

We can now identify the elements in $H_{H_{0}}^{*}(\mathcal{N})$, which pull back to the $H$-equivariant classes on $N$ represented by $[\Omega-\mathrm{i}\langle\mu, \phi\rangle]$ and $\left[-\frac{1}{2}(\phi, \phi)\right]$ in (6.30). Namely, we have the correspondences

$$
\begin{aligned}
\Omega-\mathrm{i}\langle\mu, \phi\rangle & \stackrel{\mathrm{pr}^{*}}{\longleftrightarrow} \Omega-\mathrm{i}\left(\gamma_{0}, \psi\right), \\
-\frac{1}{2}(\phi, \phi) & \stackrel{\mathrm{pr}^{*}}{\longleftrightarrow} \Theta-\frac{1}{2}(\psi, \psi) .
\end{aligned}
$$

We abuse notation slightly in the first line of (6.35), since on the left $\Omega$ is the symplectic form on $X$, whereas on the right $\Omega$ is the symplectic form on $\mathcal{M}$. The identification between these equivariant cohomology classes of degree-two is manifest once we recall that the value of the moment map $\mu$ on $\mathcal{M}$ is given dually by $\gamma_{0}$.

The identification between the equivariant classes of degree-four in the second line of (6.35) will be similarly transparent as soon as we define $\Theta$ as a characteristic class in $H^{*}(\mathcal{M})$. Specifically, we once again consider the fiber 
bundle $\bar{N}$ in (6.33). By assumption, $H$ acts on $\bar{N}$ with stabilizer $H_{0}$, so $\bar{N}$ is not the total space of a principal $H$-bundle over $\mathcal{M}$. However, if we let $K$ be the maximal subgroup of $H$ commuting with $H_{0}$, so that $K \times H_{0} \subseteq H$, then $K$ does act freely on $\bar{N}$, and we regard $\bar{N}$ as a principal $K$-bundle over $\mathcal{M}$. We then define $\Theta$ to be the degree-four characteristic class of $\bar{N}$ which is determined via the Chern-Weil homomorphism by the restriction of $-\frac{1}{2}(\phi, \phi)$ to the Lie algebra of $K$. The correspondence in the second line of (6.35) then follows directly by definition of $\Theta$.

With the identifications in (6.35), we see how the classes $\left[\Omega-\mathrm{i}\left(\gamma_{0}, \psi\right)\right]$ and $\left[\Theta-\frac{1}{2}(\psi, \psi)\right]$ in (6.31) appear under the reduction from (6.30). From a physical perspective, these terms in the localization formula arise by classical reduction.

Conversely, the ratio of equivariant Euler classes in (6.31) arises by integrating the localization form $\exp (s D \Psi)$ over the directions $\left(\mathfrak{h} \ominus \mathfrak{h}_{0}\right) \times F$ in (6.30). As in the Duistermaat-Heckman formula [11,46], where an equivariant Euler class makes its appearance for much the same reason, this prefactor can be described physically as a ratio of one-loop determinants associated to the normal directions to $\mathcal{C}$ in $X$.

Since we will need to compute the analogues of $e_{H_{0}}\left(\mathcal{M}, E_{0}\right)$ and $e_{H_{0}}(\mathcal{M}$, $\left.E_{1}\right)$ for the Seifert loop operator in Section 7, let us recall a more concrete description of the equivariant Euler class. We let $E$ be any complex representation of $H_{0}$, which is fibered over $\mathcal{M}$ to determine an $H_{0}$-equivariant bundle (with $H_{0}$ acting trivially on $\mathcal{M}$ as throughout). The $H_{0}$-equivariant Euler class $e_{H_{0}}(\mathcal{M}, E)$ then incorporates both the algebraic data associated to the action of $H_{0}$ on $E$ as well as the topological data that describes the twisting of $E$ over $\mathcal{M}$.

To encode the algebraic data related to the action of $H_{0}$ on $E$, we assume without loss that $H_{0}$ is abelian. (Otherwise, we simply restrict to a maximal torus in $H_{0}$.) We then decompose $E$ under the action of $H_{0}$ into a sum of one-dimensional complex eigenspaces

$$
E=\bigoplus_{j=1}^{\operatorname{dim} E} E_{\alpha_{j}}
$$

where each $\alpha_{j}$ is a weight in $\mathfrak{h}_{0}^{*}$ which describes the action of $H_{0}$ on the eigenspace $E_{\alpha_{j}}$.

To encode similarly the topological data associated to the vector bundle determined by $E$ over $\mathcal{N}$, we apply the splitting principle in topology, as explained for instance in Ch. 21 of [25] and as illustrated at the conclusion 
of Section 5.2. By this principle, we can assume that the vector bundle determined by $E$ over $\mathcal{M}$ splits equivariantly into a sum of line-bundles associated to each of the eigenspaces $E_{\alpha_{j}}$ for the action of $H_{0}$. Under this assumption, we let $e_{j}=c_{1}\left(E_{\alpha_{j}}\right)$ be the first Chern class of the corresponding line-bundle. These virtual Chern roots $e_{j}$ determine the total Chern class of $E$ as

$$
c(E)=\prod_{j=1}^{\operatorname{dim} E}\left(1+e_{j}\right) .
$$

In particular, the ordinary Euler class of $E$ over $\mathcal{M}$ is given by

$$
e(\mathcal{M}, E)=\prod_{j=1}^{\operatorname{dim} E} e_{j} .
$$

The equivariant Euler class $e_{H_{0}}(\mathcal{M}, E)$ is now determined in terms of the weights $\alpha_{j}$ and the Chern roots $e_{j}$. We recall that $e_{H_{0}}(\mathcal{N}, E)$ is defined as an element of $H_{H_{0}}^{*}(\mathcal{M}, E)=H^{*}(\mathcal{M}) \otimes H_{H_{0}}^{*}(p t)$. Thus $e_{H_{0}}(\mathcal{M}, E)$ will be a function of $\psi \in \mathfrak{h}_{0}$ with values in the ordinary cohomology ring of $\mathcal{M}$. Very briefly, the necessary function is given by the product

$$
e_{H_{0}}(\mathcal{M}, E)=\prod_{j=1}^{\operatorname{dim} E}\left(\frac{i\left\langle\alpha_{j}, \psi\right\rangle}{2 \pi}+e_{j}\right) .
$$

When $H_{0}$ acts trivially on $E$ so that all $\alpha_{j}$ vanish, we see that this expression for $e_{H_{0}}(\mathcal{M}, E)$ reduces to the ordinary Euler class in (6.38). At the opposite extreme, when $\mathcal{M}$ is only a point, the Chern roots $e_{j}$ do not appear in (6.39) for dimensional reasons, and the product over the weights $\alpha_{j}$ in (6.39) reduces to the determinant of $[\psi / 2 \pi]$ acting on $E$.

\subsubsection{Two special cases}

To conclude our description of the non-abelian localization formula in (6.31), let us mention two particularly simple special cases.

At one extreme, we suppose that $H$ acts freely on a neighborhood of the vanishing locus $\mathcal{C}=\mu^{-1}(0) \subset X$ of the moment map $\mu$. Thus $H_{0}$ is trivial, and $\gamma_{0}=E_{0}=E_{1}=0$. As first shown by Witten in [108], the nonabelian localization formula in this case reduces to the following integral over $\mathcal{M}=\mu^{-1}(0) / H$,

$$
\left.Z(\epsilon)\right|_{\mathcal{M}}=\int_{\mathcal{M}} \exp [\Omega+\epsilon \Theta] .
$$


Here $\Theta$ is now the degree-four characteristic class associated to $\mu^{-1}(0)$, regarded as a principal $H$-bundle over $\mathcal{M}$, and determined under the ChernWeil homomorphism by $-\frac{1}{2}(\phi, \phi)$.

At the opposite extreme, we allow the stabilizer $H_{0} \subset H$ to be non-trivial, but we assume that $\mathcal{M}$ is simply a point. The non-abelian localization formula for $\left.Z\right|_{\mathcal{M}}$ in (6.31) then reduces to an integral over the Lie algebra $\mathfrak{h}_{0}$,

$$
\begin{aligned}
\left.Z(\epsilon)\right|_{\mathcal{M}}= & \frac{1}{\operatorname{Vol}\left(H_{0}\right)} \int_{\mathfrak{h}_{0}}\left[\frac{d \psi}{2 \pi}\right] \operatorname{det}\left(\left.\frac{\psi}{2 \pi}\right|_{E_{0}}\right) \operatorname{det}\left(\left.\frac{\psi}{2 \pi}\right|_{E_{1}}\right)^{-1} \\
& \times \exp \left[-\mathrm{i}\left(\gamma_{0}, \psi\right)-\frac{\epsilon}{2}(\psi, \psi)\right] .
\end{aligned}
$$

Here, we have written the $H_{0}$-equivariant Euler classes in (6.31) more explicitly as determinants of $\psi \in \mathfrak{h}_{0}$ acting on the respective vector spaces $E_{0}$ and $E_{1}$.

The expression for $\left.Z\right|_{\mathcal{M}}$ in (6.41) makes clear a necessary caveat in our general localization formula. If $E_{0}$ and $E_{1}$ are finite-dimensional representations of $H_{0}$, the ratio of determinants in (6.41) is just a ratio of invariant polynomials on $\mathfrak{h}_{0}$. Thus the integral over $\mathfrak{h}_{0}$ in (6.41) is clearly convergent at large $\psi$, due to the exponential suppression of the integrand when $\epsilon$ is nonzero. However, depending upon $E_{0}$ and $E_{1}$, the integrand in (6.41) might also have singularities at points in $\mathfrak{h}_{0}$ for which the determinant of $\psi$ acting on $E_{1}$ vanishes. For instance, the determinant of $\psi$ acting on $E_{1}$ always vanishes at $\psi=0$, and without a compensating zero from the determinant of $\psi$ acting on $E_{0}$, the integral over $\mathfrak{h}_{0}$ could fail to converge near the origin. So strictly speaking, the integral over $\mathfrak{h}_{0}$ in the non-abelian localization formula (6.31) is not convergent in general.

We discussed this issue at some length in Section 4.3 and Appendix A of [18]. To summarize the results of that discussion, the non-abelian localization formula is valid whenever the integral over $\mathfrak{h}_{0}$ turns out to be convergent. In all our applications to Chern-Simons theory, this condition will hold.

\subsection{Elementary examples}

To provide some additional intuition for the localization formula in (6.31), let us discuss a couple of elementary examples in which the canonical symplectic integral can be evaluated directly and the result compared to (6.31). In the first example, we will illustrate the role of $E_{0}$ in the local model for $F$. In the second example, we will illustrate the role of $E_{1}$. 


\subsubsection{Symplectic volume of a coadjoint orbit}

In our first example, we consider the canonical symplectic integral defined for $X=\mathcal{O}_{\lambda}$, the adjoint orbit of $G$ through $\lambda \in \mathfrak{t}$ introduced in Section 4.1. Here, the role of the Hamiltonian group $H$ acting on $X$ is played by $G$ itself. We recall that the coadjoint symplectic form $\nu_{\lambda}$ on $\mathcal{O}_{\lambda}$ is given by

$$
\nu_{\lambda}=-\frac{1}{2}(\theta,[\lambda, \theta]), \quad \theta=g^{-1} d g
$$

and the moment map $\mu$ that describes the action of $G$ on $\mathcal{O}_{\lambda}$ is given by

$$
\langle\mu, \phi\rangle=\left(g \lambda g^{-1}, \phi\right)=-\operatorname{Tr}\left[\left(g \lambda g^{-1}\right) \cdot \phi\right], \quad \phi \in \mathfrak{g} .
$$

Trivially, the norm-square $(\mu, \mu)=(\lambda, \lambda)$ is constant on $\mathcal{O}_{\lambda}$. As a result, the canonical symplectic integral $Z(\epsilon)$ in (6.1) is proportional to the symplectic volume of $\mathcal{O}_{\lambda}$,

$$
\begin{aligned}
Z(\epsilon) & =\frac{1}{\operatorname{Vol}(G)}\left(\frac{1}{2 \pi \epsilon}\right)^{\Delta_{G} / 2} \exp \left[-\frac{(\lambda, \lambda)}{2 \epsilon}\right] \cdot \int_{\mathcal{O}_{\lambda}} \mathrm{e}^{\nu_{\lambda}} \\
\Delta_{G} & =\operatorname{dim} G .
\end{aligned}
$$

By convention, we take $\lambda>0$ to lie in the positive Weyl chamber, and we orient $\mathcal{O}_{\lambda}$ so that the symplectic volume is positive. Then from the description of $\nu_{\lambda}$ in (6.42),

$$
\int_{\mathcal{O}_{\lambda}} \mathrm{e}^{\nu_{\lambda}}=\frac{\operatorname{Vol}(G)}{\operatorname{Vol}\left(G_{\lambda}\right)} \prod_{\langle\beta, \lambda\rangle>0}\langle\beta, \lambda\rangle, \quad \beta \in \mathfrak{R}
$$

Here, $G_{\lambda} \subseteq G$ is the stabilizer of $\lambda$ under the adjoint action, and the product in (6.45) runs over roots $\beta$ of $G$ whose associated rootspaces lie in the holomorphic tangent space $\mathfrak{g}^{(1,0)}$ to $\mathcal{O}_{\lambda}$ defined in (4.23). As usual, this product arises after diagonalizing the adjoint action of $\lambda$ on $\mathfrak{g}^{(1,0)}$, such that $\lambda$ acts on each rootspace $\mathfrak{e}_{\beta} \subset \mathfrak{g}^{(1,0)}$ by $\left[\lambda, \mathfrak{e}_{\beta}\right]=\mathrm{i}\langle\beta, \lambda\rangle \mathfrak{e}_{\beta}$. Finally, we recall that the Riemannian volumes $\operatorname{Vol}(G)$ and $\operatorname{Vol}\left(G_{\lambda}\right)$ are both defined with respect to the invariant form $(\cdot, \cdot)$ determined by ' $-\mathrm{Tr}$ ' on the Lie algebra $\mathfrak{g}$. 
Thus according to (6.44) and (6.45), the canonical symplectic integral in this example is given directly by

$$
Z(\epsilon)=\frac{1}{\operatorname{Vol}\left(G_{\lambda}\right)}\left(\frac{1}{2 \pi \epsilon}\right)^{\Delta_{G} / 2} \exp \left[-\frac{(\lambda, \lambda)}{2 \epsilon}\right] \cdot \prod_{\langle\beta, \lambda\rangle>0}\langle\beta, \lambda\rangle .
$$

We want to compare (6.46) to what we obtain when we apply the nonabelian localization formula. To describe $\mathcal{O}_{\lambda}$ in terms of the local symplectic model for $F$, we set

$$
H=G, \quad H_{0}=G_{\lambda}, \quad \gamma_{0}=\lambda, \quad E_{0}=\mathfrak{g}^{(1,0)}, \quad E_{1}=\{0\} .
$$

With these assignments, $F$ in (6.21) reduces to $\mathcal{O}_{\lambda}$ itself, and the space $\mathcal{M}$ sitting beneath $F$ in the symplectic fibration (6.20) is just a point.

Since $\mathcal{M}$ is only a point, the localization formula in (6.41) presents $Z(\epsilon)$ as an integral over the Lie algebra $\mathfrak{g}_{\lambda}$ of $G_{\lambda}$,

$$
Z(\epsilon)=\frac{1}{\operatorname{Vol}\left(G_{\lambda}\right)} \int_{\mathfrak{g}_{\lambda}}\left[\frac{d \psi}{2 \pi}\right] \operatorname{det}\left(\left.\frac{\psi}{2 \pi}\right|_{\mathfrak{g}^{(1,0)}}\right) \exp \left[-\mathrm{i}(\lambda, \psi)-\frac{\epsilon}{2}(\psi, \psi)\right]
$$

Just as in (6.45), the determinant of $\psi \in \mathfrak{g}_{\lambda}$ acting via the adjoint action on $\mathfrak{g}^{(1,0)}$ is given by a product over roots $\beta$ of $G$,

$$
\begin{aligned}
\operatorname{det}\left(\left.\frac{\psi}{2 \pi}\right|_{\mathfrak{g}^{(1,0)}}\right) & =\left(\frac{\mathrm{i}}{2 \pi}\right)^{\left(\Delta_{G}-\Delta_{G_{\lambda}}\right) / 2} \cdot \prod_{\langle\beta, \lambda\rangle>0}\langle\beta, \psi\rangle, \\
\Delta_{G} & =\operatorname{dim} G, \quad \Delta_{G_{\lambda}}=\operatorname{dim} G_{\lambda} .
\end{aligned}
$$

As a result,

$$
\begin{aligned}
Z(\epsilon)= & \frac{1}{\operatorname{Vol}\left(G_{\lambda}\right)}\left(\frac{\mathrm{i}}{2 \pi}\right)^{\left(\Delta_{G}-\Delta_{G_{\lambda}}\right) / 2} \int_{\mathfrak{g}_{\lambda}}\left[\frac{d \psi}{2 \pi}\right] \\
& \times \prod_{\langle\beta, \lambda\rangle>0}\langle\beta, \psi\rangle \exp \left[-\mathrm{i}(\lambda, \psi)-\frac{\epsilon}{2}(\psi, \psi)\right] .
\end{aligned}
$$


To evaluate the integral over $\mathfrak{g}_{\lambda}$ in (6.50), we employ the standard trick to rewrite $Z(\epsilon)$ as the derivative of a Gaussian function,

$$
\begin{aligned}
Z(\epsilon)= & \frac{1}{\operatorname{Vol}\left(G_{\lambda}\right)}\left(\frac{\mathrm{i}}{2 \pi}\right)^{\left(\Delta_{G}-\Delta_{G_{\lambda}}\right) / 2} \int_{\mathfrak{g}_{\lambda}}\left[\frac{d \psi}{2 \pi}\right] \\
& \times \prod_{\langle\beta, \lambda\rangle>0}\left(\beta, \mathrm{i} \frac{\partial}{\partial \lambda}\right) \exp \left[-\mathrm{i}(\lambda, \psi)-\frac{\epsilon}{2}(\psi, \psi)\right] \\
= & \frac{1}{\operatorname{Vol}\left(G_{\lambda}\right)}\left(\frac{\mathrm{i}}{2 \pi}\right)^{\left(\Delta_{G}-\Delta_{G_{\lambda}}\right) / 2} \cdot\left[\prod_{\langle\beta, \lambda\rangle>0}\left(\beta, \mathrm{i} \frac{\partial}{\partial \lambda}\right)\right] \mathrm{I}(\lambda, \epsilon),
\end{aligned}
$$

where $\mathrm{I}(\lambda, \epsilon)$ is the Gaussian function obtained from the first line of (6.51) once the derivatives are pulled outside the integral,

$$
\mathrm{I}(\lambda, \epsilon)=\left(\frac{1}{2 \pi \epsilon}\right)^{\Delta_{G_{\lambda}} / 2} \exp \left[-\frac{(\lambda, \lambda)}{2 \epsilon}\right]
$$

When the differential operator $\prod_{\langle\beta, \lambda\rangle>0}(\beta$, i $\partial / \partial \lambda)$ acts on $\mathrm{I}(\lambda, \epsilon)$, we obtain two sorts of terms. First, each derivative with respect to $\lambda$ can act separately on the argument of the exponential in $\mathrm{I}(\lambda, \epsilon)$ to bring down a factor $-\mathrm{i}\langle\beta, \lambda\rangle / \epsilon$. Alternatively, successive derivatives in the operator $\prod_{\langle\beta, \lambda\rangle>0}(\beta, \mathrm{i} \partial / \partial \lambda)$ can act on the monomial prefactor in $\lambda$, which is generated by preceding derivatives. So schematically,

$$
\begin{aligned}
& {\left[\prod_{\langle\beta, \lambda\rangle>0}\left(\beta, \mathrm{i} \frac{\partial}{\partial \lambda}\right)\right] \mathrm{I}(\lambda, \epsilon)} \\
& \quad=\left(\frac{1}{\mathrm{i} \epsilon}\right)^{\left(\Delta_{G}-\Delta_{G_{\lambda}}\right) / 2}\left[\prod_{\langle\beta, \lambda\rangle>0}\langle\beta, \lambda\rangle+\mathcal{O}(\epsilon)\right] \cdot \mathrm{I}(\lambda, \epsilon),
\end{aligned}
$$

where $\mathcal{O}(\epsilon)$ indicates polynomial terms of higher order in $\epsilon$ which arise $a$ priori in the second way. In the case $G=S U(3)$, we present the $\mathcal{O}(\epsilon)$ terms explicitly below.

Via (6.53), the expression for $Z(\epsilon)$ in $(6.51)$ becomes

$$
Z(\epsilon)=\frac{1}{\operatorname{Vol}\left(G_{\lambda}\right)}\left(\frac{1}{2 \pi \epsilon}\right)^{\Delta_{G} / 2} \exp \left[-\frac{(\lambda, \lambda)}{2 \epsilon}\right] \cdot\left[\prod_{\langle\beta, \lambda\rangle>0}\langle\beta, \lambda\rangle+\mathcal{O}(\epsilon)\right] .
$$


Comparing (6.54) to (6.46), we see that the product over $\beta$ in the righthand bracket produces precisely the same expression for $Z(\epsilon)$ that we found previously by direct integration.

That said, consistency of (6.54) with (6.46) also requires the $\mathcal{O}(\epsilon)$ terms in (6.53) to vanish identically. Rather than attempt a general proof of this statement, we will content ourselves to check it in the simplest non-trivial case, for which $G=S U(3)$ and $\lambda=\mathrm{i} \operatorname{diag}\left(\lambda_{1}, \lambda_{2}, \lambda_{3}\right)$ is regular. The $\mathcal{O}(\epsilon)$ terms in (6.54) are then proportional to the following cyclic sum over the three positive roots $\beta_{1}, \beta_{2}$, and $\beta_{3}=\beta_{1}+\beta_{2}$ of $S U(3)$,

$\sum\left(\beta_{i}, \beta_{j}\right)\left\langle\beta_{k}, \lambda\right\rangle=\left(\beta_{1}, \beta_{2}\right)\left\langle\beta_{3}, \lambda\right\rangle+\left(\beta_{3}, \beta_{1}\right)\left\langle\beta_{2}, \lambda\right\rangle+\left(\beta_{2}, \beta_{3}\right)\left\langle\beta_{1}, \lambda\right\rangle$. $(i, j, k)$ cyclic

As standard,

$$
\left\langle\beta_{1}, \lambda\right\rangle=\lambda_{1}-\lambda_{2}, \quad\left\langle\beta_{2}, \lambda\right\rangle=\lambda_{2}-\lambda_{3}, \quad\left\langle\beta_{3}, \lambda\right\rangle=\lambda_{1}-\lambda_{3} .
$$

Also, the pairwise inner products of the roots in the invariant metric " $-\operatorname{Tr}(\cdot)$ " on $\mathfrak{s u}(3)$ are given by

$$
\left(\beta_{1}, \beta_{2}\right)=-1, \quad\left(\beta_{2}, \beta_{3}\right)=1, \quad\left(\beta_{1}, \beta_{3}\right)=1 .
$$

The cyclic sum in (6.55) then vanishes trivially according to (6.56) and (6.57), as required by the non-abelian localization formula. ${ }^{24}$

\subsubsection{Non-abelian localization on $S^{2}$}

For our second example, we take $X=S^{2}$. The discussion here will be a slight elaboration on the example provided by Witten in the appendix of [108]. We parameterize $S^{2}$ globally in terms of angular coordinates $(\theta, \varphi)$, where $\theta \in[0, \pi]$ is the latitude and $\varphi \in[0,2 \pi)$ is the longitude. As standard, we take the symplectic form $\Omega$ to be

$$
\Omega=\sin \theta d \theta \wedge d \varphi
$$

As a special case of the previous example, we considered the transitive action of $S U(2)$ on $S^{2}$, interpreted as a coadjoint orbit of $S U(2)$. Here we consider a different group action on $S^{2}$, which will not be transitive. So we take $H=U(1)$ to act by rotations on $S^{2}$, with fixed points at the poles

\footnotetext{
${ }^{24}$ I have also checked directly that the $\mathcal{O}(\epsilon)$ terms in (6.53) vanish when $\lambda$ is regular and the group is $G_{2}$, a computation which is considerably more involved.
} 
$\theta=\{0, \pi\}$. This $U(1)$ action is generated by the vector field $V=\partial / \partial \varphi$, with moment map

$$
\mu=\cos \theta+\mu_{0},
$$

where $\mu_{0}$ is an arbitrary constant. As hopefully clear, we regard the moment map as taking values in $\mathfrak{u}(1)^{*} \cong \mathfrak{u}(1)=\mathbb{R}$. For convenience, we set $\mu_{0}=0$ in the following.

By convention, we orient $S^{2}$ so that $\Omega$ is positive, and we normalize the metric on $U(1)$ so that $\operatorname{Vol}(U(1))=2 \pi$. The canonical symplectic integral $Z(\epsilon)$ then becomes

$$
\begin{aligned}
Z(\epsilon) & =\frac{1}{\operatorname{Vol}(U(1))} \frac{1}{\sqrt{2 \pi \epsilon}} \int_{S^{2}} \exp \left[\Omega-\frac{1}{2 \epsilon}(\cos \theta)^{2}\right], \\
& =\frac{1}{\sqrt{2 \pi \epsilon}} \int_{-1}^{1} d x \exp \left[-\frac{x^{2}}{2 \epsilon}\right] .
\end{aligned}
$$

In passing to the second line of (6.60), we integrate trivially over $\varphi$ to obtain a factor of $2 \pi$, which cancels the volume of $U(1)$, and we make the substitution $x=\cos \theta$. Because the integration variable $x$ ranges only over the finite interval $[-1,1], Z(\epsilon)$ is given by the Gaussian error-function and has no simple analytic expression in terms of more elementary functions.

Once again, we want to compare the result for $Z(\epsilon)$ in (6.60) with what we obtain from the non-abelian localization formula.

The local contributions to $Z(\epsilon)$ arise from critical points of the function

$$
S=\frac{1}{2}(\mu, \mu)=\frac{1}{2}(\cos \theta)^{2} .
$$

These critical points are located along the equator $\theta=\pi / 2$, where the moment map vanishes, as well as the poles $\theta=\{0, \pi\}$, where the moment map takes the values \pm 1 . So under localization, $Z(\epsilon)$ can be expressed as a sum of three terms,

$$
Z(\epsilon)=\left.Z(\epsilon)\right|_{\theta=\pi / 2}+\left.Z(\epsilon)\right|_{\theta=0}+\left.Z(\epsilon)\right|_{\theta=\pi},
$$

each of which we now compute.

We first compute the contribution from the equator, where the moment map vanishes. In a neighborhood of the equator, $U(1)$ acts freely, so the special case of the localization formula in (6.40) is applicable. Specifically, 
in terms of the local symplectic fibration (6.20), the symplectic fiber $F$ is the cotangent bundle $T^{*} U(1)$, and the base $\mathcal{M}$ is a point. So trivially,

$$
\left.Z(\epsilon)\right|_{\theta=\pi / 2}=\int_{\mathcal{M}=\{p t\}} \exp [\Omega+\epsilon \Theta]=1 .
$$

Equivalently, as will be useful in comparing to (6.60), we rewrite the constant ' 1 ' as

$$
\left.Z(\epsilon)\right|_{\theta=\pi / 2}=\frac{1}{\sqrt{2 \pi \epsilon}} \int_{-\infty}^{+\infty} d x \exp \left[-\frac{x^{2}}{2 \epsilon}\right]
$$

More interesting are the local contributions from the higher critical points at $\theta=\{0, \pi\}$. Both critical points make an identical contribution to $Z(\epsilon)$, so we need only compute the contribution from $\theta=0$. In this case, the point $\theta=0$ is fixed under the $U(1)$ action, and the symplectic fiber $F=\mathbb{C}$ in (6.20) is the tangent space to $S^{2}$ that point. To describe $F$ in terms of the canonical local model, we set ${ }^{25}$

$$
H=H_{0}=U(1), \quad \gamma_{0}=i, \quad E_{0}=\{0\}, \quad E_{1}=\mathbb{C} .
$$

Since $\mathcal{M}$ is now a point with a non-trivial stabilizer, we apply the special case of the localization formula in (6.41) to obtain

$$
\left.Z(\epsilon)\right|_{\theta=0}=\frac{1}{\operatorname{Vol}(U(1))} \int_{\mathbb{R}}\left[\frac{d \psi}{2 \pi}\right] \operatorname{det}\left(\left.\frac{\psi}{2 \pi}\right|_{\mathbb{C}}\right)^{-1} \exp \left[-\mathrm{i} \psi-\frac{\epsilon}{2} \psi^{2}\right]
$$

Here, we normalize $\psi \in \mathfrak{u}(1)$ to act with unit weight on $E_{1}=\mathbb{C}$. Thus

$$
\operatorname{det}\left(\left.\frac{\psi}{2 \pi}\right|_{\mathbb{C}}\right)=-\frac{\mathrm{i} \psi}{2 \pi}
$$

The minus sign on the right in (6.67) is a consequence of the convention for complex structures on $E_{0}$ and $E_{1}$ in (6.23). Indeed, this example provides a check of the relative sign for $\gamma_{0}$ in (6.23).

Recalling that $\operatorname{Vol}(U(1))=2 \pi$ in (6.66), we are left to analyze the integral

$$
\left.Z(\epsilon)\right|_{\theta=0}=\int_{\mathbb{R}}\left[\frac{d \psi}{2 \pi}\right] \mathrm{i} \psi^{-1} \exp \left[-\mathrm{i} \psi-\frac{\epsilon}{2} \psi^{2}\right] .
$$

The existence of the integral in (6.68) is rather delicate, due to the singularity in the integrand at $\psi=0$. To make sense of (6.68), we follow the discussion

\footnotetext{
${ }^{25}$ By convention, the generator $\gamma_{0} \in \mathfrak{u}(1)$ is anti-Hermitian and related to $\mu$ by $\gamma_{0}=\mathrm{i} \mu$.
} 
surrounding (4.90) in [18] and consider instead the derivative of $Z(\epsilon)$ with respect to $\epsilon$, for which the same non-abelian localization formula implies

$$
\begin{aligned}
\left.\frac{\partial Z(\epsilon)}{\partial \epsilon}\right|_{\theta=0} & =-\frac{\mathrm{i}}{2} \int_{\mathbb{R}}\left[\frac{d \psi}{2 \pi}\right] \psi \exp \left[-\mathrm{i} \psi-\frac{\epsilon}{2} \psi^{2}\right], \\
& =-\frac{1}{2 \sqrt{2 \pi}} \epsilon^{-3 / 2} \exp \left[-\frac{1}{2 \epsilon}\right]
\end{aligned}
$$

In passing to the second line of (6.69), we evaluate the integral over $\psi$ after completing the square in the argument of the exponential.

The result in (6.69) suffices to determine $\left.Z(\epsilon)\right|_{\theta=0}$ as a function of $\epsilon$ up to an arbitrary integration constant $c_{0}$,

$$
\begin{aligned}
\left.Z(\epsilon)\right|_{\theta=0} & =-\frac{1}{2 \sqrt{2 \pi}} \int_{0}^{\epsilon} d y y^{-3 / 2} \exp \left[-\frac{1}{2 y}\right]+c_{0}, \\
& =-\frac{1}{\sqrt{2 \pi \epsilon}} \int_{1}^{\infty} d x \exp \left[-\frac{x^{2}}{2 \epsilon}\right]+c_{0},
\end{aligned}
$$

where in the second line of (6.70), we substitute $x=\sqrt{\epsilon / y}$. The same argument shows that $\left.Z(\epsilon)\right|_{\theta=\pi}$ is given by an identical expression, which we write as

$$
\left.Z(\epsilon)\right|_{\theta=\pi}=-\frac{1}{\sqrt{2 \pi \epsilon}} \int_{-\infty}^{-1} d x \exp \left[-\frac{x^{2}}{2 \epsilon}\right]+c_{0}
$$

For $c_{0}=0$, the sum over the local contributions from the critical points in $(6.64),(6.70)$, and (6.71) then exactly reproduces the error-function in $(6.60)$.

\section{Localization for Seifert loops in Chern-Simons theory}

We are finally prepared to apply non-abelian localization to the Seifert loop path integral, which as in Section 4 takes the canonical form

$$
Z(\epsilon ; C, R)=\frac{1}{\operatorname{Vol}(\mathcal{G})}\left(\frac{-\mathrm{i}}{2 \pi \epsilon}\right)^{\Delta_{\mathcal{G}} / 2} \int_{\overline{\mathcal{A}}_{\alpha}} \exp \left[\Omega_{\alpha}+\frac{\mathrm{i}}{2 \epsilon}(\mu, \mu)\right]
$$

By the general properties of the canonical symplectic integral, $Z(\epsilon ; C, R)$ localizes onto the critical points in $\overline{\mathcal{A}}_{\alpha}=\overline{\mathcal{A}} \times \epsilon L \mathcal{O}_{\alpha}$ of the shift-invariant 
action

$$
S(A, U)=\operatorname{cs}(A)+2 \epsilon \oint_{C} \operatorname{Tr}\left(\alpha \cdot g^{-1} d_{A} g\right)-\int_{M} \frac{1}{\kappa \wedge d \kappa} \operatorname{Tr}\left[\left(\kappa \wedge \mathcal{F}_{A}\right)^{2}\right],
$$

where

$$
\mathcal{F}_{A}=F_{A}+\epsilon\left(g \alpha g^{-1}\right) \delta_{C} .
$$

Thus our first task in analyzing the path integral in (7.1) is to characterize the critical points of $S(A, U)$.

Varying $S(A, U)$ in (7.2) with respect to $A$, we immediately find one classical equation of motion,

$$
F_{A}+\epsilon\left(g \alpha g^{-1}\right) \delta_{C}-\left(\frac{\kappa \wedge \mathcal{F}_{A}}{\kappa \wedge d \kappa}\right) d \kappa-\kappa \wedge d_{A}\left(\frac{\kappa \wedge \mathcal{F}_{A}}{\kappa \wedge d \kappa}\right)=0
$$

Varying with respect to $g$, we find the other equation of motion,

$$
\left[\alpha, g^{-1} d_{A} g-\kappa g^{-1}\left(\frac{\kappa \wedge \mathcal{F}_{A}}{\kappa \wedge d \kappa}\right) g\right]=0
$$

To avoid cluttering the notation, we have suppressed an overall factor of $\delta_{C}$ in (7.5), so the equation of motion derived by varying $g$ must be understood to hold upon restriction to $C$.

Since we have yet to fix a gauge for the shift symmetry $\mathcal{S}$, the equations of motion in (7.4) and (7.5) are invariant under the transformation $\delta A=\sigma \kappa$, with $\sigma$ being an arbitrary function on $M$ valued in the Lie algebra $\mathfrak{g}$ of the gauge group $G$. To fix a gauge for $\mathcal{S}$, we observe that the quantity $\kappa \wedge \mathcal{F}_{A}$ transforms very simply under the shift of $A$,

$$
\kappa \wedge \mathcal{F}_{A} \longmapsto \kappa \wedge \mathcal{F}_{A}+\sigma \kappa \wedge d \kappa
$$

Since $\kappa \wedge d \kappa$ is a non-vanishing three-form on $M$, we can then unambiguously fix the shift symmetry by the condition

$$
\kappa \wedge \mathcal{F}_{A}=0
$$

In this gauge, the shift-invariant equations of motion in (7.4) and (7.5) reduce to

$$
\mathcal{F}_{A}=F_{A}+\epsilon\left(g \alpha g^{-1}\right) \cdot \delta_{C}=0, \quad\left[\alpha, g^{-1} d_{A} g\right]=0,
$$


or in terms of $U=g \alpha g^{-1}$,

$$
F_{A}+\epsilon U \cdot \delta_{C}=0, \quad d_{A} U=0 .
$$

We have seen these equations before, precisely as (4.62) and (4.63) in Section 4.2. There we noted that solutions to (7.9) correspond to classical configurations for the fields $A$ and $U$ in the background of a Wilson loop operator in the standard (non-shift-invariant) formulation of Chern-Simons theory. Hence, the Seifert loop path integral localizes onto the moduli space $\mathcal{M}(C, \alpha)$ of connections, which are flat on the complement to $C$ in $M$ and otherwise have delta-function curvature along $C$ determined by the covariantly constant value of $U$.

\subsection{More about the classical Seifert loop operator}

For generic $k \in \mathbb{Z}$ and $\alpha \in \Gamma_{\mathrm{wt}}$, we recall the explicit description of $\mathcal{M}(C, \alpha)$ as a moduli space of homomorphisms from the fundamental group of the knot complement $M^{o}=M-C$ to $G$,

$$
\begin{aligned}
\mathcal{M}(C, \alpha) & =\left\{\varrho^{o}: \pi_{1}\left(M^{o}\right) \rightarrow G \mid \varrho^{o}(\mathrm{~m}) \in \mathfrak{C}_{\alpha / k}\right\} / G, \\
\mathfrak{C}_{\alpha / k} & =\operatorname{Cl}[\exp (2 \pi \alpha / k)], \quad \mathrm{m}=\text { meridian of } C .
\end{aligned}
$$

The description of $\mathcal{M}(C, \alpha)$ in (7.10) holds for general $M$ and $C$, but for the localization computations to be performed in Sections 7.2 and 7.3, we must specialize to the Seifert case. Not surprisingly, when $C \subset M$ is Seifert, the classical moduli space $\mathcal{M}(C, \alpha)$ acquires additional geometric structure. This structure will be essential for the cohomological interpretation of the Seifert loop operator, and it is what we wish to explore now.

According to (7.10), the form of $\mathcal{M}(C, \alpha)$ is largely determined by the fundamental group $\pi_{1}\left(M^{o}\right)$, so let us quickly present $\pi_{1}\left(M^{o}\right)$ when $C \subset M$ is Seifert. As in Section 3.2, we suppose that the Seifert manifold $M$ is characterized by the Seifert invariants

$$
\left[h ; n ;\left(a_{1}, b_{1}\right), \ldots,\left(a_{N}, b_{N}\right)\right], \quad \operatorname{gcd}\left(a_{j}, b_{j}\right)=1 .
$$

Here $h$ is the genus of the Riemann surface $\Sigma$ which sits at the base of $M$, $n$ is the degree of the Seifert fibration over $\Sigma$, and the relatively-prime pairs $\left(a_{j}, b_{j}\right)$ for $j=1, \ldots, N$ specify the topology of $M$ over each orbifold point of $\Sigma$. 
"ATMP-17-1-A1-BEA" — 2013/5/15 - 19:32 — page 119 — \#119

As we briefly discussed in Section 5.1 of [18], the fundamental group $\pi_{1}(M)$ of the closed Seifert manifold $M$ with Seifert invariants (7.11) is generated by elements

$$
\begin{array}{ll}
\mathrm{a}_{\ell}, \mathrm{b}_{\ell}, & \ell=1, \ldots, h, \\
\mathrm{c}_{j}, & j=1, \ldots, N, \\
\mathrm{f}, &
\end{array}
$$

subject to the following relations:

$$
\begin{aligned}
{\left[\mathrm{a}_{\ell}, \mathrm{f}\right] } & =\left[\mathrm{b}_{\ell}, \mathrm{f}\right]=\left[\mathrm{c}_{j}, \mathrm{f}\right]=1, \\
\mathrm{c}_{j}^{a_{j}} \mathrm{f}^{b_{j}} & =1, \\
\prod_{\ell=1}^{h}\left[\mathrm{a}_{\ell}, \mathrm{b}_{\ell}\right] \prod_{j=1}^{N} \mathrm{c}_{j} & =\mathrm{f}^{n}, \quad\left[\mathrm{a}_{\ell}, \mathrm{b}_{\ell}\right] \equiv \mathrm{a}_{\ell} \mathrm{b}_{\ell} \mathrm{a}_{\ell}^{-1} \mathrm{~b}_{\ell}^{-1} .
\end{aligned}
$$

The generator $\mathrm{f}$, which is a central element in $\pi_{1}(M)$ by the first line of (7.13), arises geometrically from the $S^{1}$ fiber over $\Sigma$. Similarly, the generators $a_{\ell}$ and $b_{\ell}$ arise from the $2 h$ non-contractible cycles present when $\Sigma$ is a smooth Riemann surface of genus $h$. Finally, for $N>0$ the surface $\Sigma$ carries a non-trivial orbifold structure, and the generators $\mathrm{c}_{j}$ for $j=1, \ldots, N$ correspond to small one-cycles around each of the orbifold points of $\Sigma$.

To present the Seifert loop moduli space $\mathcal{M}(C, \alpha)$, we actually want the fundamental group of the complement $M^{o}=M-C$, as opposed to $M$ itself. However, when $C$ is a generic Seifert fiber of $M$, the presentation of $\pi_{1}(M)$ in (7.12) and (7.13) can be immediately extended to a presentation of $\pi_{1}\left(M^{o}\right)$. We simply adjoin a single generator $m$ to the set in (7.12), where, consistent with the previous notation, $m$ represents the meridian of $C$. As for relations, $m$ commutes with the fiber $\mathrm{f}$ (which is represented geometrically by $C$ itself),

$$
[\mathrm{m}, \mathrm{f}]=1,
$$

and the last relation in (7.13) must be modified to include $\mathrm{m}$ as

$$
\prod_{\ell=1}^{h}\left[\mathrm{a}_{\ell}, \mathrm{b}_{\ell}\right] \prod_{j=1}^{N} \mathrm{c}_{j}=\mathrm{m} \mathrm{f}^{n} .
$$

To explain (7.15) briefly, we assume that $C$ sits as the fiber over a smooth, non-orbifold point $p \in \Sigma$. Then $\mathrm{m}$ is represented geometrically by a small, suitably oriented one-cycle around $p$ in $\Sigma$. Hence, $m$ appears in (7.15) in the same way that the generators $c_{j}$ enter the corresponding relation in (7.13). 
"ATMP-17-1-A1-BEA" — 2013/5/15 — 19:32 — page 120 — \#120

\subsubsection{A two-dimensional interpretation for $\mathcal{M}(C, \alpha)$}

The preceding presentation for $\pi_{1}\left(M^{o}\right)$ is clearly reminiscent of the presentation for $\pi_{1}\left(\Sigma^{o}\right)$ in Section 5.2, where $\Sigma^{o}=\Sigma-\{p\}$ is the punctured Riemann surface at the base of $M^{o}$. Indeed, the presentation for $\pi_{1}\left(M^{o}\right)$ directly reduces to the presentation for $\pi_{1}\left(\Sigma^{o}\right)$ once we eliminate the generator $f \in \pi_{1}\left(M^{o}\right)$, setting $f=1$ in the relations (7.13), (7.14), and (7.15). Here when $\Sigma$ has orbifold points, we take $\pi_{1}\left(\Sigma^{o}\right)$ to be the orbifold fundamental group. Hence as groups, $\pi_{1}\left(M^{o}\right)$ is a central extension of $\pi_{1}\left(\Sigma^{o}\right)$,

$$
1 \longrightarrow \mathbb{Z} \longrightarrow \pi_{1}\left(M^{o}\right) \longrightarrow \pi_{1}\left(\Sigma^{o}\right) \longrightarrow 1,
$$

where $f$ generates $\mathbb{Z}$ above.

The relationship between the groups $\pi_{1}\left(M^{o}\right)$ and $\pi_{1}\left(\Sigma^{o}\right)$ in $(7.16)$ is naturally reflected in a corresponding relationship between the moduli spaces $\mathcal{M}(C, \alpha)$ and $\mathcal{N}(P ; \lambda)$, where we recall from Section 5.2 that $\mathcal{N}(P ; \lambda)$ describes flat connections on $\Sigma$ with monodromy at $p$ specified by $\lambda \in \mathfrak{t}$. In the special case $\alpha=\lambda=0$, that relationship reduces to a correspondence between the respective moduli spaces $\mathcal{N}$ and $\mathcal{N}(P)$ of (non-singular) flat connections on $M$ and $\Sigma$, as we discussed in Section 5.1 of [18] following much earlier observations by Furuta and Steer [53]. Since the twodimensional interpretation of $\mathcal{M}(C, \alpha)$ fundamentally underlies our work on non-abelian localization, let us briefly review the observations in [18] as applied to $\mathcal{M}(C, \alpha)$.

To explain the relationship between $\mathcal{M}(C, \alpha)$ and $\mathcal{N}(P ; \lambda)$, we consider a fixed homomorphism $\varrho^{o}: \pi_{1}\left(M^{o}\right) \rightarrow G$, where we assume $G$ to be simplyconnected. Since $f$ is central in $\pi_{1}\left(M^{o}\right)$, the image of $\varrho^{o}$ must lie in the centralizer $\mathcal{Z}_{\varrho^{o}(\mathrm{f})} \subseteq G$ of the element $\varrho^{o}(\mathrm{f}) \in G$. To simplify the following discussion, we suppose that $\varrho^{o}(\mathrm{f})$ actually lies in the center $\mathcal{Z}(G)$ of $G$, implying $\mathcal{Z}_{\varrho^{o}(\mathrm{f})}=G$. This condition is necessary whenever the connection described by $\varrho^{o}$ is irreducible, which is one of the two main cases we consider when we perform computations for the Seifert loop operator. In the irreducible case, we will take $\Sigma$ to have genus $h \geq 1$. For the other case, we consider, $\Sigma$ has genus zero, and we do not require $\varrho^{o}(\mathrm{f})$ to be central nor $\varrho^{o}$ irreducible. As often happens, the genus zero case is somewhat special, and we postpone consideration of it until the end of this section.

Clearly if $\varrho^{o}(f)=1$, so that the corresponding flat connection on $M^{o}$ has trivial holonomy around the Seifert fiber, then $\varrho^{o}$ factors through the extension (7.16) to determine a homomorphism $\varrho^{o}: \pi_{1}\left(\Sigma^{o}\right) \rightarrow G$. As in (5.1) and (5.2), the parameters $\alpha$ and $\lambda$, which specify the respective monodromies 
"ATMP-17-1-A1-BEA" — 2013/5/15 — 19:32 — page 121 — \#121

for connections in $\mathcal{M}(C, \alpha)$ and $\mathcal{N}(P ; \lambda)$ are related by

$$
\lambda=\frac{\alpha}{k} .
$$

Under the identification in (7.17), $\varrho^{\circ}$ specifies a flat connection with monodromy on $M$ which is merely the pullback from a corresponding flat connection with monodromy on $\Sigma$.

Otherwise, if $\varrho^{o}(\mathrm{f})$ is a non-trivial element in the center $\mathcal{Z}(G)$, then the flat connection (with monodromy) on $M$ has non-trivial holonomy around the Seifert fiber and cannot be the pullback of a flat $G$-connection (with monodromy) on $\Sigma$. However, if we pass from the simply-connected group $G$ to the quotient $G_{\text {ad }}=G / \mathcal{Z}(G)$, so that we consider the connection on $M$ as a connection on the trivial $G_{\text {ad }}$-bundle, then the holonomy of this connection around the Seifert fiber becomes trivial. As a result, the homomorphism $\varrho^{o}$ can now be interpreted as describing a flat connection with monodromy on $M$ that arises as the pullback from a corresponding flat connection on a generally non-trivial $G_{\mathrm{ad}}$-bundle $P$ over $\Sigma$. Specializing for convenience to the case that $\Sigma$ is smooth, with no orbifold points, we see by comparing the relation (7.15) in $\pi_{1}\left(M^{o}\right)$ to the relation $(5.26)$ in $\pi_{1}\left(\Sigma^{o o}\right)$ that the holonomy $\varrho^{o}(\mathrm{f})$ is related to the central element $\zeta \in \mathcal{Z}(G)$ which characterizes the topology of the principal $G_{\text {ad }}$-bundle $P$ on $\Sigma$ via

$$
\zeta=\varrho^{o}(\mathrm{f})^{n}
$$

For instance, if we consider the case that the gauge group $G$ is $S U(2)$ and the degree $n$ of $M$ as a principal $U(1)$-bundle over $\Sigma$ is odd, then flat connections on $M$ whose holonomies satisfy $\varrho^{o}(\mathrm{f})=\varrho^{o}(\mathrm{f})^{n}=-1$ correspond bijectively to flat $S U(2)$ connections on $\Sigma$ which have monodromy -1 around a specified puncture. Such flat $S U(2)$ connections can then be identified with flat connections on the topologically non-trivial principal $S O(3)$-bundle over $\Sigma$. On the other hand, if the degree $n$ is even, then $\varrho^{o}(\mathrm{f})^{n}=1$ for both $\varrho^{o}(f)= \pm 1$, so points in both of these components of the moduli space on $M$ are identified with flat $S U(2)$ connections on $\Sigma$.

If $\Sigma$ is not smooth but is an orbifold, the prior discussion extends immediately when additional monodromies on $\Sigma$ associated to the elements $f^{\beta_{\ell}}$ in (7.13) are similarly taken into account.

Let us make a concluding parenthetical remark. In obtaining a twodimensional interpretation for the irreducible homomorphism $\varrho^{o}$ on $M^{o}$, we naturally pass from the simply-connected group $G$ to the quotient $G_{\mathrm{ad}}=$ $G / \mathcal{Z}(G)$. If we correctly account for this quotient, the corresponding smooth 
component $\mathcal{M}_{0}(C, \alpha)$ in the full moduli space $\mathcal{M}(C, \alpha)$ is identified most literally with the unramified cover $\widetilde{\mathcal{N}}(P ; \lambda) \rightarrow \mathcal{N}(P ; \lambda)$ appearing in (5.30),

$$
\mathcal{M}_{0}(C, \alpha) \cong \tilde{\mathcal{N}}(P ; \lambda), \quad \lambda=\alpha / k
$$

as opposed to the moduli space $\mathcal{N}(P ; \lambda)$ itself. When $\alpha=\lambda=0$, a similar statement holds for smooth components $\mathcal{M}_{0}$ in the moduli space $\mathcal{M}$ of flat connections on $M$,

$$
\mathcal{M}_{0} \cong \tilde{\mathcal{N}}(P)
$$

Nonetheless, as we noted following (5.30), the distinction between $\mathcal{N}(P ; \lambda)$ and the finite cover $\widetilde{\mathcal{N}}(P ; \lambda)$ in $(7.19)$ will not be essential for our work.

\subsubsection{Consequences for the symplectic geometry of $\mathcal{M}(C, \alpha)$}

According to discussion in Section 5.2, when $P$ and $\lambda$ are appropriately chosen, the extended moduli space $\mathcal{N}(P ; \lambda)$ is a smooth manifold which fibers symplectically over the more basic moduli space $\mathcal{N}(P)$ of flat connections on $P$,

$$
2 \pi \mathcal{O}_{-\lambda} \longrightarrow \mathcal{N}(P ; \lambda)
$$

However, as we reviewed just a moment ago, $\mathcal{N}(P ; \lambda)$ also bears a natural relation to $\mathcal{M}(C, \alpha)$ when $C \subset M$ is Seifert. Via the identification in (7.19), the fibration in (7.21) implies a corresponding fibration for suitable components of $\mathcal{M}(C, \alpha)$ over the moduli space $\mathcal{M}$ of flat connections on $M$,

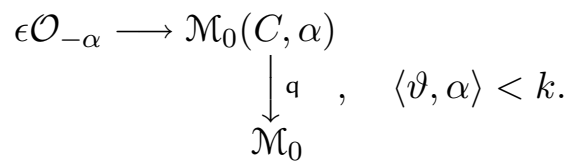

In obtaining (7.22), we recall the identification $\lambda=\alpha / k$ and the definition $\epsilon=2 \pi / k$. Also, both $\mathcal{M}_{0}$ and $\mathcal{M}_{0}(C, \alpha)$ denote connected components of the typically disconnected moduli spaces $\mathcal{M}$ and $\mathcal{N}(C, \alpha)$. In general, the fibration in (7.22) holds for only some components of $\mathcal{M}(C, \alpha)$, since only some components of $\mathcal{N}(C, \alpha)$ are smooth. For the application in Section 7.3 , we will be most interested in the case that $\Sigma$ is a smooth Riemann surface of genus $h \geq 1$ (with no orbifold points), and the central holonomy 
$\varrho^{o}(\mathrm{f}) \in \mathcal{Z}(G)$ around the Seifert fiber of $M$ is such that the associated component $\mathcal{M}_{0}(C, \alpha)$ is smooth. In that situation, the fibration in (7.22) does hold.

Just as for (7.21), the symplectic form (4.104) on $\overline{\mathcal{A}}_{\alpha}$ induces a symplectic form $\Omega_{\alpha}$ on $\mathcal{M}_{0}(C, \alpha)$ which is compatible with the fibration in (7.22), so that $\Omega_{\alpha}$ admits a decomposition

$$
\Omega_{\alpha}=\mathrm{q}^{*} \Omega-\epsilon \mathrm{e}_{\alpha}, \quad \epsilon=2 \pi / k .
$$

Here, $\Omega$ is the symplectic form on $\mathcal{M}_{0}$ induced from the corresponding form (3.20) on $\overline{\mathcal{A}}$, and $e_{\alpha}$ is a closed two-form on $\mathcal{M}_{0}(C, \alpha)$ which restricts fiberwise to the coadjoint symplectic form on $\mathcal{O}_{\alpha}$. Of course, the description of $\Omega_{\alpha}$ in (7.23) is nothing more than the transcription of (5.32) to the Seifert setting.

In Section 5.2, we were careful to consider the dependence of $\mathcal{N}(P ; \lambda)$ on the parameter $\lambda \in \mathfrak{t}$ and to delineate the regime of validity for the fiberwise symplectic decomposition in (5.32). For instance, due to singular gauge transformations taking the form (5.4), we saw that the affine Weyl group $\mathfrak{W}_{\text {aff }}$ of $G$ acts as a group of discrete gauge symmetries on $\lambda$. We then fixed the residual action of $\mathfrak{W}_{\text {aff }}$ on $\lambda$ by requiring $\lambda$ to lie in the fundamental Weyl alcove $D_{+} \subset \mathfrak{t}$. Briefly, we recall that $D_{+}$is the bounded region in $\mathfrak{t}$ specified by the inequalities $\lambda \geq 0$ and $\langle\vartheta, \lambda\rangle \leq 1$, where $\vartheta$ is the highest root of $G$. Finally, we observed that the symplectic fibration in (7.21) is valid away from the wall of $D_{+}$defined by $\langle\vartheta, \lambda\rangle=1$, where the symplectic form on $\mathcal{N}(P ; \lambda)$ degenerates.

Under the identification $\lambda=\alpha / k$, precisely the same conclusions apply to $\mathcal{M}_{0}(C, \alpha)$. Gauge transformations on $M$ which are singular along $C$, again of the form (5.4), alter the local behavior (5.1) of $A$ near $C$ by shifting

$$
\alpha \longmapsto \alpha+k y, \quad y \in \Gamma_{\mathrm{rt}},
$$

for $y$ an arbitrary element in the root lattice $\Gamma_{\mathrm{rt}}$ of the simply-connected group $G$. This discrete gauge symmetry can be fixed by taking the weight $\alpha$ to lie in $k \cdot \mathrm{D}_{+}$, implying that $\alpha$ satisfies the bounds $\alpha \geq 0$ and $(\vartheta, \alpha) \leq k .^{26}$ Finally, the symplectic fibration for $\mathcal{M}_{0}(C, \alpha)$ in (7.22) is valid for positive weights $\alpha$ which satisfy the strict inequality $(\vartheta, \alpha)<k$.

\footnotetext{
${ }^{26}$ For once, we regard both the root $\vartheta$ and the weight $\alpha$ as elements of $\mathfrak{t}^{*}$. The pairing between $\vartheta$ and $\alpha$ is then defined using the invariant metric $(\cdot, \cdot)$ on $\mathfrak{t}^{*}$ induced by ' $-\operatorname{Tr}$ '.
} 
In the context of Chern-Simons theory, the discrete gauge symmetry (7.24) is especially interesting, since it is the manifestation of a well-known quantum mechanical equivalence among the set of all Wilson loop operators in the theory. As well-known, in the classical limit $k \rightarrow \infty$, the Wilson loop operators $W_{R}(C)$ defined for a fixed curve $C$ are distinct operators in ChernSimons theory as $R$ ranges over the set of irreducible representations of $G$. Yet if $k<\infty$ is finite, a quantum mechanical basis [47] for the set of Wilson loops wrapping $C$ is given by only the finite set of operators associated to those representations, which are integrable in the affine Lie algebra $\hat{\mathfrak{g}}$ at level $k$. Explicitly, the integrable representations of $\hat{\mathfrak{g}}$ at level $k$ are characterized by the bound (see for instance Section 14.3.1 of [40])

$$
(\vartheta, \alpha) \leq k,
$$

where $\alpha \geq 0$ is the highest weight of $R$. In terms of $\mathfrak{t}^{*} \cong \mathfrak{t}$, the integrability condition in (7.25) simply says that $\alpha$ lies in the bounded region $k \cdot \mathrm{D}_{+}$, as we already observed.

In the literature, one sometimes finds the integrability condition (7.25) alternatively expressed by means of the strict inequality

$$
(\vartheta, \alpha+\rho)<k+\check{c}_{\mathfrak{g}},
$$

where $\rho$ is the distinguished weight which is half the sum of the positive roots of $G$,

$$
\rho=\frac{1}{2} \sum_{\beta>0} \beta, \quad \beta \in \Re,
$$

and $\check{c}_{\mathfrak{g}}$ is the dual Coxeter number of $G$. For instance, if $G=S U(r+1)$, then $\check{c}_{\mathfrak{g}}=r+1$. The strict form (7.26) of the integrability condition on the weight $\alpha \geq 0$ is entirely equivalent to the condition in (7.25), due to the standard identity $(\vartheta, \rho)=\check{c}_{\mathfrak{g}}-1$.

For the semi-classical computations in Section 7.3, we will need to assume that $\alpha \geq 0$ satisfies the strict bound

$$
(\vartheta, \alpha)<k
$$

so that the symplectic fibration in (7.22) is valid. This bound is slightly stronger than the integrability condition in (7.25), which is the appropriate bound on $\alpha$ in the quantum theory.

Nonetheless, the bound (7.25) on $\alpha$ derived from two-dimensional conformal field theory and the bound (7.28) on $\alpha$ derived from symplectic geometry 
are not unrelated. In its strict form (7.26), the integrability condition can be very naturally interpreted as the quantum analogue of the classical constraint in (7.28). As often happens for Chern-Simons theory, in passing from the classical to the quantum bound, the level $k$ is replaced by $k+\check{c}_{\mathfrak{g}}$, corresponding for $G=S U(2)$ to the infamous shift $k \mapsto k+2$, and the weight $\alpha$ itself is replaced by $\alpha+\rho$. Eventually in the course of the localization computations in Sections 7.2 and 7.3, we will see how both of these rather delicate quantum corrections arise.

\subsubsection{Analogue for Seifert homology spheres}

The symplectic fibration $(7.22)$ for $\mathcal{M}(C, \alpha)$ is most relevant when $\Sigma$ has genus $h \geq 1$, in which case smooth components $\mathcal{M}_{0}$ and $\mathcal{M}_{0}(C, \alpha)$ of positive dimension generally exist. Yet before we proceed to computations, we also wish to consider the structure of $\mathcal{M}(C, \alpha)$ in the opposite topological regime, for which $\Sigma$ has genus zero and $M$ is a Seifert homology sphere.

To start, let us recall what it means for $M$ to be a Seifert homology sphere. In general, a compact three-manifold $M$ is a homology sphere if and only if $H_{1}(M)=0$. Furthermore, $H_{1}(M)$ is always the abelianization of $\pi_{1}(M)$. Given the explicit description of $\pi_{1}(M)$ in (7.12) and (7.13), we can easily characterize the conditions on the Seifert invariants (7.11) of $M$ such that $H_{1}(M)$ vanishes.

First, the base $\Sigma$ of the Seifert fibration must have genus zero if $M$ is to be a homology sphere, since otherwise the homology of $\Sigma$ would contribute to the homology of $M$. Specializing to this case in (7.12) and (7.13), the fundamental group $M$ is then generated by the elements $\mathrm{c}_{j}$ for $j=1, \ldots, N$ and $\mathrm{f}$, subject to the relations

$$
\begin{aligned}
{\left[\mathrm{c}_{j}, \mathrm{f}\right] } & =1, \quad j=1, \ldots, N, \\
\mathrm{c}_{j}^{a_{j}} \mathrm{f}^{b_{j}} & =1, \\
\prod_{j=1}^{N} \mathrm{c}_{j} & =\mathrm{f}^{n} .
\end{aligned}
$$

The vanishing of $H_{1}(M)$ implies that the relations in (7.29) must be nondegenerate in the following sense. To make the notation more uniform, let us set $\mathrm{f} \equiv \mathrm{c}_{N+1}$. Then $H_{1}(M)$ is the abelian group generated by the $\mathrm{c}_{j}$ for $j=1, \ldots, N+1$, subject to $N+1$ relations of the form $\prod_{j=1}^{N+1} c_{j}^{\mathbf{K}_{j, l}}=1$, where $\mathbf{K}_{j, l}$ is an $(N+1) \times(N+1)$ integer-valued matrix derived from the last two lines of (7.29). An arbitrary element $\prod_{j=1}^{N+1} c_{j}^{v_{j}}$ in $H_{1}(M)$ is trivial if and only if the vector $v_{j}$ can be written as $v_{j}=\sum_{l} \mathbf{K}_{j, l} w_{l}$ for some other 
vector $w_{l}$ of integers. In terms of $\mathbf{K}_{j, l}$, the vanishing of $H_{1}(M)$ is then equivalent to the condition $\operatorname{det} \mathbf{K}= \pm 1$. As one can easily verify, the latter requirement amounts to the arithmetic condition that the Seifert invariants (7.11) of $M$ satisfy

$$
n+\sum_{j=1}^{N} \frac{b_{j}}{a_{j}}= \pm \prod_{j=1}^{N} \frac{1}{a_{j}} .
$$

Hence if $h=0$ and the arithmetic condition in (7.30) holds, $M$ is a Seifert homology sphere.

As in Section 5.2 of [18], the present discussion will actually apply to a slightly more general class of three-manifolds than Seifert homology spheres. To indicate the broader class of Seifert manifolds we consider, we note that the quantity appearing on the left of (7.30) is the Chern class of the line $V$-bundle $\mathcal{L}$ over $\Sigma$ associated to $M$,

$$
c_{1}(\mathcal{L})=n+\sum_{j=1}^{N} \frac{b_{j}}{a_{j}}>0,
$$

where we assume without loss that $M$ is oriented so that $c_{1}(\mathcal{L})$ is positive. If the arithmetic condition on $c_{1}(\mathcal{L})$ in $(7.30)$ holds, then the orders $a_{j}$ of the orbifold points on $\Sigma$ are necessarily pairwise coprime,

$$
\operatorname{gcd}\left(a_{j}, a_{j^{\prime}}\right)=1, \quad j \neq j^{\prime},
$$

and as explained for instance in Section 1 of [53], $\mathcal{L}$ generates the Picard group of line $V$-bundles over $\Sigma$. That is, every line $V$-bundle on $\Sigma$ is an integral multiple of $\mathcal{L}$.

Besides the Seifert homology sphere associated to the fundamental line $V$-bundle $\mathcal{L}$, we also consider the Seifert manifolds which arise from any multiple $\mathcal{L}^{d}$ with $d \geq 1$. The Seifert manifold $M$ derived from $\mathcal{L}^{d}$ is a quotient by the cyclic group $\mathbb{Z}_{d}$ of the integral homology sphere associated to $\mathcal{L}$, and in this case, $H_{1}(M)=\mathbb{Z}_{d}$. Thus, the integer $d$ can be characterized topologically as the order of $H_{1}(M)$,

$$
d=\left|H_{1}(M)\right|
$$

Such Seifert manifolds are rational homology spheres, with $H_{1}(M ; \mathbb{R})=0$. As a simple example, if $\mathcal{L}$ is the smooth line-bundle of degree one over $\mathbb{C P}^{1}$, which describes $S^{3}$, then the rational homology spheres associated to multiples $\mathcal{L}^{d}$ are lens spaces. 
Regardless of whether $M$ is a Seifert homology sphere or a cyclic quotient thereof, the vanishing of $H_{1}(M ; \mathbb{R})$ implies that the trivial connection is isolated as a flat connection on $M$. Hence the trivial connection determines a distinguished point $\{0\} \in \mathcal{M}$, whose contribution to the Chern-Simons path integral is natural to consider under localization.

One can ask whether an analogous statement holds for the extended moduli space $\mathcal{M}(C, \alpha)$. That is, if $M$ is a Seifert homology sphere or a cyclic quotient thereof, does $\mathcal{M}(C, \alpha)$ contain an distinguished, isolated point $\left\{\varrho_{*}\right\} \in \mathcal{M}(C, \alpha)$, whose local contribution to the Seifert loop path integral is equally natural to consider? As our notation indicates, $\varrho_{*}: \pi_{1}\left(M^{o}\right) \rightarrow G$ must provide a generally non-trivial representation of the knot group $\pi_{1}\left(M^{o}\right)$, since $\varrho_{*}(\mathrm{~m})$ lies by assumption in the generally non-trivial conjugacy class $\mathfrak{C}_{\alpha / k}=\mathrm{Cl}[\exp (2 \pi \alpha / k)]$.

To answer this question, we will directly exhibit $\varrho_{*}$. We do so by the time-tested means of following our nose.

Specializing to the case of Seifert homology spheres, we first observe that the knot group $\pi_{1}\left(M^{o}\right)$ is generated by elements $\mathrm{c}_{j}, \mathrm{f}$, and $\mathrm{m}$, subject to the relations

$$
\begin{aligned}
{[\mathrm{m}, \mathrm{f}] } & =\left[\mathrm{c}_{j}, \mathrm{f}\right]=1, \quad j=1, \ldots, N, \\
\mathrm{c}_{j}^{a_{j}} \mathrm{f}^{b_{j}} & =1, \\
\prod_{j=1}^{N} \mathrm{c}_{j} & =\mathrm{m} \mathrm{f}^{n} .
\end{aligned}
$$

According to (7.10), if $\varrho_{*}$ determines a point in $\mathcal{M}(C, \alpha)$, then $\varrho_{*}(\mathrm{~m})=$ $\exp \left(2 \pi U_{0} / k\right)$ for some $U_{0} \in \mathcal{O}_{\alpha}$. Without loss, we similarly parameterize the values of $\varrho_{*}$ on the generators $\mathrm{c}_{1}, \ldots, \mathrm{c}_{N}$ and $f \equiv c_{N+1}$ in terms of elements $\delta_{j} \in \mathfrak{g}$, such that $\varrho_{*}\left(\mathbf{c}_{j}\right)=\exp \left(\delta_{j}\right)$ for $j=1, \ldots, N+1$.

So far, we have made no assumptions about $\varrho_{*}$. However, as the analogue of $\{0\} \in \mathcal{M}$, the homomorphism $\varrho_{*}$ should be suitably 'close' to the trivial homomorphism, for which $\delta_{j}=0$ for all $j=1, \ldots, N+1$. Motivated by this observation, we initially work to first order in the parameters $\left(\delta_{1}, \ldots, \delta_{N+1}\right)$ in our attempt to construct $\varrho_{*}$.

At first order, the relations among the generators in (7.34) imply corresponding linear relations among the parameters $\left(\delta_{1}, \ldots, \delta_{N+1}\right)$ which specify $\varrho_{*}$. Specifically, if $\mathbf{K}_{j, l}$ is the $(N+1) \times(N+1)$ integer-valued matrix that appeared following (7.29) in our discussion of $H_{1}(M)$, then $\sum_{j} \mathbf{K}_{j, l} \delta_{j}=0$ for $l=1, \ldots, N$, and $\sum_{j} \mathbf{K}_{j, l} \delta_{j}=2 \pi U_{0} / k$ for $l=N+1$. Since $M$ is a 
"ATMP-17-1-A1-BEA" — 2013/5/15 — 19:32 — page 128 — \#128

Seifert homology sphere or a cyclic quotient thereof, the matrix $\mathbf{K}_{j, l}$ is nondegenerate over $\mathbb{R}$. Therefore, if $U_{0} \in \mathcal{O}_{\alpha}$ is held fixed, the inhomogeneous linear system for the parameters $\left(\delta_{1}, \ldots, \delta_{N+1}\right)$ has a unique solution. Moreover, by linearity, each $\delta_{j}$ for $j=1, \ldots, N+1$ is proportional to $U_{0}$ as an element of $\mathfrak{g}$. Explicitly, in terms of the inverse matrix $\left(\mathbf{K}^{-1}\right)_{l, j}$, we have $\delta_{j}=\left(\mathbf{K}^{-1}\right)_{N+1, j} \cdot\left(2 \pi U_{0} / k\right)$. In particular, the elements $\left(\delta_{1}, \ldots, \delta_{N+1}\right)$ all commute, and we obtain an honest homomorphism $\varrho_{*}: \pi_{1}\left(M^{o}\right) \rightarrow G$, even though we began by working only to first order in $\left(\delta_{1}, \ldots, \delta_{N+1}\right)$.

Necessarily $\varrho_{*}$ is a reducible representation of $\pi_{1}\left(M^{o}\right)$, since $\varrho_{*}$ is invariant under constant gauge transformations on $M$ which preserve $U_{0}$. Such gauge transformations form a subgroup of $G$ conjugate to the stabilizer $G_{\alpha}$. Also, any infinitesimal deformation of $\varrho_{*}$ with fixed $U_{0}$ must lie in the kernel of $\mathbf{K}_{j, l}$. But since $\mathbf{K}_{j, l}$ is non-degenerate over $\mathbb{R}$, the kernel of $\mathbf{K}_{j, l}$ trivial. Therefore, like the trivial flat connection on $M$, the reducible connection described by $\varrho_{*}$ is isolated as a flat connection on $M^{o}$ with monodromy $\varrho_{*}(\mathrm{~m})$ fixed by $U_{0} \in \mathcal{O}_{\alpha}$. Since $\varrho_{*}$ derives from a maximally reducible, abelian representation of $\pi_{1}\left(M^{o}\right)$, we henceforth set $\varrho_{*} \equiv \varrho_{\mathrm{ab}}$ to indicate this fact.

We are not quite done, though, since the parameter $U_{0}$ still varies in the orbit $\mathcal{O}_{\alpha}$. As $U_{0}$ varies, the homomorphism $\varrho_{\text {ab }}$ also varies, so we actually have a family of homomorphisms parameterized by $\mathcal{O}_{\alpha}$. However, by the same token, constant gauge transformations on $M$ induce the adjoint action of $G$ on $\mathcal{O}_{\alpha}$. Thus in total, $\left\{\varrho_{\mathrm{ab}}\right\}$ represents a distinguished point in $\mathcal{M}(C, \alpha)$ isomorphic to the quotient $\mathcal{O}_{\alpha} / G$,

$$
\left\{\varrho_{\mathrm{ab}}\right\} \cong \mathcal{O}_{\alpha} / G \in \mathcal{M}(C, \alpha)
$$

Despite the fact that $\left\{\varrho_{\mathrm{ab}}\right\} \cong \mathcal{O}_{\alpha} / G$ is naively just a point, keeping track of both the orbit $\mathcal{O}_{\alpha}$ and the $G$-action on $\mathcal{O}_{\alpha}$ will be extremely important for the localization computation in Section 7.2. To illustrate why such an equivariant perspective is useful, let us consider the analogue for Seifert homology spheres of the smooth fibration in (7.22).

If $\alpha=0$, the orbit $\mathcal{O}_{\alpha}$ reduces to a point, and $\varrho_{\mathrm{ab}}$ describes the trivial connection on $M^{o}$. So if $\alpha / k$ is small, the reducible connection on $M^{o}$ described by $\varrho_{\mathrm{ab}}$ is only a small deformation of the trivial connection. Via the same reasoning as in our discussion of the symplectic fibration for $\mathcal{N}(P ; \lambda)$ in Section 5.2, we then obtain the $G$-equivariant fibration

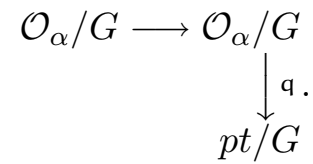


Here $\{0\} \cong p t / G$ is the point in $\mathcal{M}$ corresponding to the trivial connection on $M$. Since constant gauge transformations on $M$ fix the trivial connection, we write $\{0\} \cong p t / G$ to indicate that $\{0\}$ is equivariantly a point equipped with the trivial action of $G$.

Since the base of (7.36) is the equivariant point $p t / G$, the total space is just the equivariant fiber $\mathcal{O}_{\alpha} / G$. So admittedly, the equivariant fibration in (7.36) may appear rather formal at first glance. Nonetheless, the analogy between (7.22) and (7.36) will eventually be helpful to explain certain similarities between localization on the smooth moduli space $\mathcal{M}_{0}(C, \alpha)$ and localization at the maximally-reducible, abelian point $\left\{\varrho_{\mathrm{ab}}\right\} \cong \mathcal{O}_{\alpha} / G$.

\subsubsection{Example: torus knots in $S^{3}$}

Most intentionally, our discussion of the classical Seifert loop moduli space $\mathcal{M}(C, \alpha)$ has been couched in general terms. Oftentimes, though, concrete examples can be even more illuminating. With this thought in mind, we now turn our attention to the simplest, and also the most interesting, Seifert loop operators.

The simplest Seifert manifold is $S^{3}$, so the simplest Seifert loop operators will wrap curves embedded as knots $\mathcal{K} \subset S^{3}$. Here, we meet a basic topological question. Namely, which knots can be realized as Seifert fibers of $S^{3}$ ? Clearly, for each locally free $U(1)_{\mathrm{R}}$ action on $S^{3}$, we obtain a corresponding knot as the generic orbit. So to approach our question in a systematic fashion, let us consider the possible locally free $U(1)_{\mathrm{R}}$ actions on $S^{3}$.

We regard $S^{3}$ as the unit sphere in $\mathbb{C}^{2}$, which carries complex coordinates $(X, Y)$. For each pair of integers $(\mathbf{p}, \mathbf{q})$, we obtain a natural $U(1)$ action on $S^{3}$ under which $X$ and $Y$ transform with respective charges $\mathbf{p}$ and $\mathbf{q}$,

$$
(X, Y) \stackrel{\mathrm{e}^{\mathrm{i} \theta}}{\longmapsto}\left(\mathrm{e}^{i \mathbf{p} \theta} \cdot X, \mathrm{e}^{i \mathbf{q} \theta} \cdot Y\right), \quad \operatorname{gcd}(\mathbf{p}, \mathbf{q})=1 .
$$

Here, $\theta$ is an angular parameter on $U(1)$, and without loss we assume in (7.37) that $\mathbf{p}$ and $\mathbf{q}$ are relatively prime. Otherwise, if $\mathbf{p}$ and $\mathbf{q}$ share a common factor $f=\operatorname{gcd}(\mathbf{p}, \mathbf{q})$, then a cyclic subgroup $\mathbb{Z}_{f}$ of $U(1)$ acts trivially on $S^{3}$ and can be factored out.

The classification of locally free $U(1)_{\mathrm{R}}$ actions on $S^{3}$ is now very easy to state. So long as both $\mathbf{p}$ and $\mathbf{q}$ are non-zero, the vector field on $S^{3}$, which generates the $U(1)$ action in (7.37) is nowhere vanishing, and the action is locally free. Conversely, as for instance in Section 1.5 of [90], every locally free $U(1)_{\mathrm{R}}$ action on $S^{3}$ appears as an action of the form (7.37) with some $\mathbf{p}, \mathbf{q} \neq 0$. 
One interesting corollary of the classification of locally free $U(1)_{\mathrm{R}}$ actions on $S^{3}$ is that $S^{3}$ admits many distinct presentations as a Seifert manifold. For instance, in the case $\mathbf{p}=\mathbf{q}=1$, the $U(1)_{\mathrm{R}}$ action in (7.37) is actually free, and we obtain the standard Hopf presentation of $S^{3}$ as a smooth $S^{1}$-bundle over $\mathbb{C P}^{1}$, on which $[X: Y]$ serve as homogeneous coordinates. As we mentioned in the Introduction, here the Hopf fiber is just the unknot.

More generally, dividing $S^{3}$ by the $U(1)_{\mathrm{R}}$ action in (7.37), we obtain a fibration of $S^{3}$ over the weighted projective space $\mathbb{W} \mathbb{C P}_{\mathbf{p}, \mathbf{q}}^{1}$,

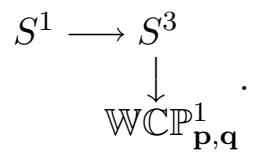

Topologically, $\mathbb{W} \mathbb{C P}_{\mathbf{p}, \mathbf{q}}^{1}$ is a genus-zero Riemann surface with two orbifold points, of orders $\mathbf{p}$ and $\mathbf{q}$. The orbifold points sit under the two exceptional orbits in $S^{3}$ where either $X$ or $Y$ vanishes, and hence a corresponding cyclic subgroup $\mathbb{Z}_{\mathbf{q}}$ or $\mathbb{Z}_{\mathbf{p}}$ in $U(1)_{\mathbf{R}}$ acts trivially.

For each Seifert presentation of $S^{3}$ in (7.38), let us quickly compute the associated Seifert invariants $\left[h ; n,\left(a_{1}, b_{1}\right),\left(a_{2}, b_{2}\right)\right]$, in the notation of $(7.11)$. Because the base $\Sigma=\mathbb{W} \mathbb{C P}_{\mathbf{p}, \mathbf{q}}^{1}$ is a genus-zero Riemann surface with orbifold points of orders $\mathbf{p}$ and $\mathbf{q}$, we obtain immediately

$$
h=0, \quad a_{1}=\mathbf{p}, \quad a_{2}=\mathbf{q} .
$$

Otherwise, the remaining Seifert invariants $\left(n, b_{1}, b_{2}\right)$ are determined by the arithmetic condition in (7.30) which characterizes Seifert homology spheres. Explicitly, the condition in (7.30) becomes

$$
n+\frac{b_{1}}{a_{1}}+\frac{b_{2}}{a_{2}}=\frac{1}{a_{1} a_{2}}, \quad 0 \leq b_{1}<a_{1}, \quad 0 \leq b_{2}<a_{2} .
$$

To solve (7.40), we note that because $a_{1}=\mathbf{p}$ and $a_{2}=\mathbf{q}$ are relatively-prime, there exist unique integers $\mathbf{r}$ and $\mathbf{s}$ such that

$$
\mathbf{p s}-\mathbf{q r}=1, \quad 0<\mathbf{r}<\mathbf{p}, \quad 0<\mathbf{s}<\mathbf{q} .
$$

In terms of $\mathbf{r}$ and $\mathbf{s}$, the unique integers $\left(n, b_{1}, b_{2}\right)$ satisfying (7.40) are then

$$
n=-1, \quad b_{1}=\mathbf{p}-\mathbf{r}, \quad b_{2}=\mathbf{s} .
$$

At this stage, from the explicit description of the $U(1)_{R}$ action in (7.37), we see immediately that the knots that can be realized as Seifert orbits in $S^{3}$ 

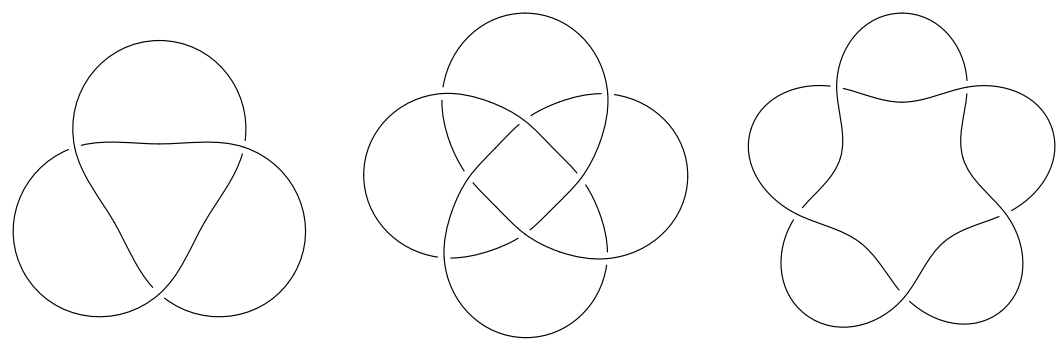

Figure 1: The torus knots $\mathcal{K}_{\mathbf{3 , 2}}, \mathcal{K}_{\mathbf{4 , 3}}$, and $\mathcal{K}_{\mathbf{5 , 2}}$.

are exactly [85] the torus knots. As the name suggests, the torus knot $\mathcal{K}_{\mathbf{p}, \mathbf{q}}$ is described by a trigonometric embedding of $S^{1}$ into a two-torus $S^{1} \times S^{1}$, itself embedded in $S^{3}$, under which

$$
\mathrm{e}^{\mathrm{i} \theta} \longmapsto\left(\mathrm{e}^{\mathrm{i} \mathbf{p} \theta}, \mathrm{e}^{\mathrm{i} \mathbf{q} \theta}\right), \quad \operatorname{gcd}(\mathbf{p}, \mathbf{q})=1 .
$$

Here, $\theta$ is an angular parameter along $S^{1}$, and $\mathbf{p}$ and $\mathbf{q}$ are again non-zero, relatively prime ${ }^{27}$ integers which determine the embedding $S^{1} \hookrightarrow S^{1} \times S^{1}$. Our reuse of $\theta$ as an angular parameter on $S^{1}$ in (7.43) is no accident. Comparing (7.37) to (7.43), we see that so long as both $X, Y \neq 0$, the torus knot $\mathcal{K}_{\mathbf{p}, \mathbf{q}}$ is embedded in $S^{3}$ as a $U(1)_{\mathrm{R}}$ orbit in the two-torus parameterized by the phases of the complex coordinates $X$ and $Y$.

For later use, let us quickly recall a few basic facts about the classification of torus knots. If either $\mathbf{p}$ or $\mathbf{q}$ is equal to 1 , then $\mathcal{K}_{\mathbf{p}, \mathbf{q}}$ is equivalent to the unknot, as we already observed for the Hopf fiber $\mathcal{K}_{\mathbf{1}, \mathbf{1}}$. Also, for fixed (p, q), the knots $\mathcal{K}_{\mathbf{p}, \mathbf{q}}$ and $\mathcal{K}_{\mathbf{q}, \mathbf{p}}$ are equivalent under isotopy in $S^{3}$. By definition, $\mathcal{K}_{-\mathbf{p},-\mathbf{q}}$ agrees with $\mathcal{K}_{\mathbf{p}, \mathbf{q}}$ but carries the opposite orientation. Finally, $\mathcal{K}_{\mathbf{p},-\mathbf{q}}$ is the mirror image of $\mathcal{K}_{\mathbf{p}, \mathbf{q}}$. All torus knots (other than the unknot) are chiral, meaning that $\mathcal{K}_{\mathbf{p}, \mathbf{q}}$ is not isotopic to its mirror. Indeed, one way to prove the latter statement is to compute the Jones polynomial for $\mathcal{K}_{\mathbf{p}, \mathbf{q}}$, as we shall do using localization in the next section.

Up to orientation and reflection, we can thus restrict attention to the torus knots associated to distinct pairs ${ }^{28} \mathbf{p}>\mathbf{q}>1$. These knots are all non-trivial and topologically distinct. By way of illustration, we present in figure 1 three torus knots, which can be drawn on the plane with few crossings, including the trefoil, which is $\mathcal{K}_{\mathbf{3}, \mathbf{2}}$. As hopefully clear, the set of

\footnotetext{
${ }^{27}$ If $\mathbf{p}$ and $\mathbf{q}$ share a common factor $f>1$, the map in (7.43) is a degree $f$ covering of the embedding determined by the reduced pair $(\mathbf{p} / f, \mathbf{q} / f)$.

${ }^{28}$ We restrict attention to positive $\mathbf{q}$ merely to suppress the proliferation of signs in the computations in Section 7.2.
} 
"ATMP-17-1-A1-BEA" — 2013/5/15 — 19:32 — page 132 — \#132

Seifert loop operators is a restricted but nonetheless quite interesting subset of the possible Wilson loop operators in $S^{3}$.

\subsubsection{Irreducible points in $\mathcal{M}\left(\mathcal{K}_{\mathrm{p}, \mathrm{q}}, \alpha\right)$}

With the torus knot $\mathcal{K}_{\mathbf{p}, \mathbf{q}}$ in hand, we finally want to make some remarks about the structure of the associated Seifert loop moduli space $\mathcal{M}\left(\mathcal{K}_{\mathbf{p}, \mathbf{q}}, \alpha\right)$. At least for gauge group $G=S U(2)$, this moduli space has been extensively studied, and our goal here is just to sketch a few of its salient features. See for instance $[23,79]$ for a much more thorough analysis.

To discuss the structure of $\mathcal{M}\left(\mathcal{K}_{\mathbf{p}, \mathbf{q}}, \alpha\right)$, we first need to discuss the structure of the knot group $\pi_{1}\left(S^{3}-\mathcal{K}_{\mathbf{p}, \mathbf{q}}\right)$. Most famously, $\pi_{1}\left(S^{3}-\mathcal{K}_{\mathbf{p}, \mathbf{q}}\right)$ is the group generated by two elements $x$ and $y$ subject to the single relation $x^{\mathbf{p}}=y^{\mathbf{q}}$. That is,

$$
\pi_{1}\left(S^{3}-\mathcal{K}_{\mathbf{p}, \mathbf{q}}\right) \cong\left\langle x, y \mid x^{\mathbf{p}}=y^{\mathbf{q}}\right\rangle
$$

When $\mathbf{p}=\mathbf{q}=1$ so that $\mathcal{K}_{\mathbf{1 , 1}}$ is the unknot, $\pi_{1}\left(S^{3}-\mathcal{K}_{\mathbf{1 , 1}}\right) \cong \mathbb{Z}$ is freely generated by $x=y$. Otherwise, for $\mathbf{p}>\mathbf{q}>1$, the knot group is a nonabelian group of infinite order.

Of course, a finitely-generated group can be presented in many different ways. Via the general description of $\pi_{1}\left(M^{\circ}\right)$ in $(7.34)$, we have already provided a distinct, Seifert set of generators and relations for the knot group in (7.44). According to our computation of the Seifert invariants in (7.39) and (7.42), the Seifert presentation of $\pi_{1}\left(S^{3}-\mathcal{K}_{\mathbf{p}, \mathbf{q}}\right)$ is given by generators $\left\{c_{1}, c_{2}, m, f\right\}$ subject to relations

$$
\begin{aligned}
{[\mathrm{m}, \mathrm{f}] } & =\left[\mathrm{c}_{j}, \mathrm{f}\right]=1, \quad j=1,2, \\
\mathrm{c}_{1}^{\mathbf{p}} \mathrm{f}^{\mathbf{p}-\mathbf{r}} & =1, \\
\mathrm{c}_{2}^{\mathbf{q}} \mathrm{f}^{\mathbf{s}} & =1 \\
\mathrm{c}_{1} \mathrm{c}_{2} & =\mathrm{mf}^{-1} .
\end{aligned}
$$

We recall from (7.41) that the coprime integers $(\mathbf{r}, \mathbf{s})$ satisfy $\mathbf{p s}-\mathbf{q r}=1$.

The presentation of $\pi_{1}\left(S^{3}-\mathcal{K}_{\mathbf{p}, \mathbf{q}}\right)$ in (7.44) is certainly simpler than the Seifert presentation in (7.45), but the Seifert presentation has the virtue of making manifest the meridian $\mathrm{m}$, which enters the definition of $\mathcal{M}\left(\mathcal{K}_{\mathbf{p}, \mathbf{q}}, \alpha\right)$. With a little bit of work, one can check that the Seifert presentation of 
$\pi_{1}\left(S^{3}-\mathcal{K}_{\mathbf{p}, \mathbf{q}}\right)$ reduces to the presentation in (7.44) under the identifications

$$
x=c_{1}^{q_{1}} c_{2}^{q_{f}} \mathrm{q}^{q}, \quad y=c_{1}^{p_{1}} c_{2}^{p_{f}} f^{p} .
$$

The meridian $m$ of the knot $\mathcal{K}_{\mathbf{p}, \mathbf{q}}$ is then specified in terms of the generators $x$ and $y$ by

$$
m=c_{1} c_{2} f=x^{-\mathbf{r}} y^{s} .
$$

For the special case of the unknot $\mathcal{K}_{\mathbf{1 , 1}}$ (with $\mathbf{r}=0, \mathbf{s}=1$ ), the meridian $\mathrm{m}=\mathrm{y}$ is the generator of $\pi_{1}\left(S^{3}-\mathcal{K}_{\mathbf{1}, \mathbf{1}}\right) \cong \mathbb{Z}$.

We are now in an excellent position to analyze the structure of the classical moduli space $\mathcal{M}\left(\mathcal{K}_{\mathbf{p}, \mathbf{q}}, \alpha\right)$, which parameterizes homomorphisms $\varrho^{o}: \pi_{1}\left(S^{3}-\mathcal{K}_{\mathbf{p}, \mathbf{q}}\right) \rightarrow G$ with monodromy $\varrho^{o}(\mathrm{~m}) \in \mathfrak{C}_{\alpha / k}$. According to the presentation of $\pi_{1}\left(S^{3}-\mathcal{K}_{\mathbf{p}, \mathbf{q}}\right)$ in $(7.44)$, any homomorphism $\varrho^{o}$ is uniquely determined by its values on the generators $x$ and $y$ of the knot group. To parameterize $\varrho^{o}$, let us therefore set

$$
\varrho^{o}(x)=X, \quad \varrho^{o}(y)=Y,
$$

for elements $\mathrm{X}, \mathrm{Y} \in G$. In terms of $\mathrm{X}$ and $\mathrm{Y}$, the moduli space $\mathcal{M}\left(\mathcal{K}_{\mathbf{p}, \mathbf{q}}, \alpha\right)$ is given very concretely by

$$
\begin{aligned}
\mathcal{M}\left(\mathcal{K}_{\mathbf{p}, \mathbf{q}}, \alpha\right) & =\left\{(\mathrm{X}, \mathrm{Y}) \in G \times G \mid \mathrm{X}^{\mathbf{p}}=\mathrm{Y}^{\mathbf{q}}, \mathrm{X}^{-\mathbf{r}} \cdot \mathrm{Y}^{\mathbf{s}} \in \mathfrak{C}_{\alpha / k}\right\} / G, \\
\mathfrak{C}_{\alpha / k} & =\operatorname{Cl}[\exp (2 \pi \alpha / k)] .
\end{aligned}
$$

As we have already observed for the general Seifert homology sphere, $\mathcal{M}\left(\mathcal{K}_{\mathbf{p}, \mathbf{q}}, \alpha\right)$ always contains a distinguished, reducible point $\left\{\varrho_{\mathrm{ab}}\right\} \cong \mathcal{O}_{\alpha} / G$, which fibers equivariantly via (7.36) over the trivial connection on $S^{3}$. To exhibit $\varrho_{\mathrm{ab}}$ in the notation of (7.49), we set

$$
X=\exp (\delta \mathrm{X}), \quad \mathrm{Y}=\exp (\delta \mathrm{Y}),
$$

where $\delta \mathrm{X}$ and $\delta \mathrm{Y}$ are elements in the Lie algebra $\mathfrak{g}$. According to (7.49), to first order the logarithms $\delta \mathrm{X}$ and $\delta \mathrm{Y}$ satisfy the linear relations

$$
\begin{aligned}
\mathbf{p} \delta \mathrm{X}-\mathbf{q} \delta \mathrm{Y} & =0, \\
-\mathbf{r} \delta \mathrm{X}+\mathbf{s} \delta \mathrm{Y} & =\frac{2 \pi}{k} U_{0}, \quad U_{0} \in \mathcal{O}_{\alpha} .
\end{aligned}
$$

If $U_{0}$ is fixed, the system in (7.51) then admits the unique solution

$$
\delta \mathrm{X}=\frac{2 \pi \mathbf{q}}{k} U_{0}, \quad \delta \mathrm{Y}=\frac{2 \pi \mathbf{p}}{k} U_{0} .
$$


Since $\delta \mathrm{X}$ and $\delta \mathrm{Y}$ are both proportional to $U_{0}$, the group elements $\mathrm{X}$ and $\mathrm{Y}$ automatically commute, and we obtain a family of abelian homomorphisms $\varrho_{\text {ab }}$ labelled by $U_{0}$. These homomorphisms correspond geometrically to the reducible point $\left\{\varrho_{\mathrm{ab}}\right\} \cong \mathcal{O}_{\alpha} / G$ in $\mathcal{M}\left(\mathcal{K}_{\mathbf{p}, \mathbf{q}}, \alpha\right)$.

If $\mathbf{p}=\mathbf{q}=1$ so that $\mathcal{K}_{\mathbf{1 , 1}}$ is the unknot, then $\mathrm{m}$ freely-generates $\pi_{1}\left(S^{3}-\right.$ $\left.\mathcal{K}_{\mathbf{1}, \mathbf{1}}\right) \cong \mathbb{Z}$, and any homomorphism $\varrho^{o}$ is entirely determined by its value on $\mathrm{m}$. Consequently, the reducible point $\left\{\varrho_{\mathrm{ab}}\right\}$ is indeed the only point in $\mathcal{M}\left(\mathcal{K}_{1,1}, \alpha\right)$.

When $\mathcal{K}_{\mathbf{p}, \mathbf{q}}$ is a non-trivial torus knot, the structure of the moduli space $\mathcal{M}\left(\mathcal{K}_{\mathbf{p}, \mathbf{q}}, \alpha\right)$ is much more interesting. Beyond the distinguished reducible point, $\mathcal{M}\left(\mathcal{K}_{\mathbf{p}, \mathbf{q}}, \alpha\right)$ may contain other points associated to irreducible ${ }^{29}$ homomorphisms $\varrho^{o}: \pi_{1}\left(S^{3}-\mathcal{K}_{\mathbf{p}, \mathbf{q}}\right) \rightarrow G$. In the case $G=S U(2)$, the existence of such points was essentially noted long ago by Klassen [79], and their presence depends upon the precise value of the parameter $\alpha / k$.

To exhibit the irreducible points in $\mathcal{M}\left(\mathcal{K}_{\mathbf{p}, \mathbf{q}}, \alpha\right)$, we first observe that $x^{\mathbf{p}}=y^{\mathbf{q}}$ is automatically a central element in the knot group $\pi_{1}\left(S^{3}-\mathcal{K}_{\mathbf{p}, \mathbf{q}}\right)$. Hence, if $\varrho^{o}$ is irreducible, the element $X^{\mathbf{p}}=Y^{\mathbf{q}}$ must lie in the center of $G$. For simplicity, we focus on the standard case $G=S U(2)$, for which $\mathrm{X}^{\mathbf{p}}=\mathrm{Y}^{\mathbf{q}}= \pm 1$. Thus, $\mathrm{X}$ and $\mathrm{Y}$ are conjugate to elements $\mathrm{X}_{0}, \mathrm{Y}_{0} \in S U(2)$ which take the diagonal forms

$$
\begin{array}{ll}
\mathrm{X}_{0}=\left(\begin{array}{cc}
\mathrm{e}^{\frac{\mathrm{i} \pi}{\mathbf{p}} u} & 0 \\
0 & \mathrm{e}^{\frac{-\mathrm{i} \pi}{\mathrm{p}} u}
\end{array}\right), \quad 0 \leq u<\mathbf{p}, \\
\mathrm{Y}_{0}=\left(\begin{array}{cc}
\mathrm{e}^{\frac{\mathrm{i} \pi}{\mathbf{q}} v} & 0 \\
0 & \mathrm{e}^{\frac{-\mathrm{i} \pi}{\mathbf{q}} v}
\end{array}\right), \quad 0 \leq v<\mathbf{q}, & v \equiv u \bmod 2,
\end{array}
$$

for some integers $u$ and $v$ which are equal modulo 2 as above.

We assume without loss that $X=X_{0}$ takes precisely the diagonal form in the first line of (7.53). Up to the action of $S U(2)$, $\mathrm{Y}$ can then be parameterized in terms of an angle $\phi$ as

$$
\mathrm{Y}=g_{\phi} \cdot \mathrm{Y}_{0} \cdot g_{\phi}^{-1}, \quad g_{\phi}=\left(\begin{array}{cc}
\cos \left(\frac{\phi}{2}\right) & i \sin \left(\frac{\phi}{2}\right) \\
i \sin \left(\frac{\phi}{2}\right) & \cos \left(\frac{\phi}{2}\right)
\end{array}\right) .
$$

\footnotetext{
${ }^{29}$ When $G$ has higher rank than $S U(2)$, the moduli space $\mathcal{M}\left(\mathcal{K}_{\mathbf{p}, \mathbf{q}}, \alpha\right)$ also generally contains points associated to partially reducible homomorphisms $\varrho^{\circ}$, whose image lies in a subgroup $G^{\prime}$ such that $T \subsetneq G^{\prime} \subsetneq G$. For the purpose of the following discussion, these partially reducible points can be lumped together with the irreducible points.
} 
The angle $\phi$ is not arbitrary but is fixed by the requirement in (7.49) that $\mathrm{X}^{-\mathbf{r}} \cdot \gamma^{\mathbf{s}}$ be conjugate to the group element $\exp (2 \pi \alpha / k)$, where the weight $\alpha$ is given explicitly by

$$
\alpha=\frac{m}{2}\left(\begin{array}{cc}
i & 0 \\
0 & -i
\end{array}\right), \quad m \in \mathbb{Z}_{\geq 0} .
$$

Tracing over the entries of the conjugate elements $\exp (2 \pi \alpha / k)$ and $\mathbf{X}^{-\mathbf{r}} \cdot \gamma^{\mathbf{s}}$ with the ansatz for $Y$ in (7.54), we obtain a trigonometric relation which determines $\phi$ in terms of the integers $(m, k, \mathbf{p}, \mathbf{q}, u, v)$,

$$
\cos \left(\frac{\pi m}{k}\right)=\cos \left(\frac{\pi u \mathbf{r}}{\mathbf{p}}\right) \cos \left(\frac{\pi v \mathbf{s}}{\mathbf{q}}\right)+\sin \left(\frac{\pi u \mathbf{r}}{\mathbf{p}}\right) \sin \left(\frac{\pi v \mathbf{s}}{\mathbf{q}}\right) \cos (\phi) .
$$

So long as $\phi$ is not equal to 0 or $\pi$, any solution of (7.56) describes an irreducible homomorphism $\varrho^{o}: \pi_{1}\left(S^{3}-\mathcal{K}_{\mathbf{p}, \mathbf{q}}\right) \rightarrow S U(2)$ with $\varrho^{o}(\mathrm{~m}) \in \mathfrak{C}_{\alpha / k}$. Geometrically, $\left\{\varrho^{o}\right\}$ corresponds to an isolated, smooth point in $\mathcal{M}\left(\mathcal{K}_{\mathbf{p}, \mathbf{q}}, \alpha\right)$, distinct from the reducible point $\left\{\varrho_{\mathrm{ab}}\right\} \cong \mathcal{O}_{\alpha} / G$.

As one example, for the trefoil knot $\mathcal{K}_{\mathbf{3 , 2}}$ (with $\mathbf{r}=\mathbf{s}=1$ ), we take $u=v=1$. Then (7.56) reduces to the condition

$$
\cos \left(\frac{\pi m}{k}\right)=\sin \left(\frac{\pi}{3}\right) \cos (\phi),
$$

which can be satisfied whenever $m / k$ lies in the interval $\left[\frac{1}{6}, \frac{5}{6}\right]$.

This example illustrates an important, well-known general phenomenon. Namely, the irreducible points in $\mathcal{M}\left(\mathcal{K}_{\mathbf{p}, \mathbf{q}}, \alpha\right)$ exist only when $\alpha / k>0$ is a finite, non-zero distance from the origin. In contrast, the maximally reducible, abelian point $\left\{\varrho_{\mathrm{ab}}\right\}$ exists even when $\alpha / k$ is arbitrarily small, as in the classical limit $k \rightarrow \infty$ with $\alpha$ held fixed. ${ }^{30}$

To summarize the discussion so far, the moduli space $\mathcal{M}\left(\mathcal{K}_{\mathbf{p}, \mathbf{q}}, \alpha\right)$ always contains the distinguished, reducible point $\left\{\varrho_{\mathrm{ab}}\right\} \cong \mathcal{O}_{\alpha} / G$. Depending on the ratio $\alpha / k$ as well as the pair $(\mathbf{p}, \mathbf{q})$, the moduli space $\mathcal{M}\left(\mathcal{K}_{\mathbf{p}, \mathbf{q}}, \alpha\right)$ may contain additional points, which arise from irreducible, non-abelian representations of the knot group $\pi_{1}\left(S^{3}-\mathcal{K}_{\mathbf{p}, \mathbf{q}}\right)$.

Under localization, the Seifert loop path integral for the torus knot $\mathcal{K}_{\mathbf{p}, \mathbf{q}}$ is generally evaluated as a sum over contributions from the connected components of $\mathcal{M}\left(\mathcal{K}_{\mathbf{p}, \mathbf{q}}, \alpha\right)$. Although evaluating the individual contribution from

\footnotetext{
More broadly, both statements are true if $\mathcal{K}$ is any knot in $S^{3}$, not necessarily a torus knot.
} 
"ATMP-17-1-A1-BEA" — 2013/5/15 — 19:32 — page 136 — \#136

each component of $\mathcal{M}\left(\mathcal{K}_{\mathbf{p}, \mathbf{q}}\right)$ is relatively straightforward (such computations are the essential content of Sections 7.2 and 7.3), summing the results over all components of $\mathcal{N}\left(\mathcal{K}_{\mathbf{p}, \mathbf{q}}\right)$ would appear to be a much harder task, requiring a detailed analysis of $\mathcal{N}\left(\mathcal{K}_{\mathbf{p}, \mathbf{q}}\right)$ for all values of $\mathbf{p}, \mathbf{q}$, and $\alpha / k$.

In fact, the situation turns out to be a good deal simpler than at first glance, and we are able to evaluate the Seifert loop path integral for the torus knot $\mathcal{K}_{\mathbf{p}, \mathbf{q}}$ entirely by localization at the distinguished reducible point $\left\{\varrho_{\mathrm{ab}}\right\} \cong \mathcal{O}_{\alpha} / G{ }^{31}$ As follows from the exact expressions obtained via conformal field theory, the Seifert loop path integral $Z\left(\epsilon ; \mathcal{K}_{\mathbf{p}, \mathbf{q}}, R\right)$ is an analytic function of the coupling $\epsilon=2 \pi / k$. Hence, the behavior of $Z\left(\epsilon ; \mathcal{K}_{\mathbf{p}, \mathbf{q}}, R\right)$ for all $\epsilon$ is determined by the behavior of $Z\left(\epsilon ; \mathcal{K}_{\mathbf{p}, \mathbf{q}}, R\right)$ for $\epsilon$ in any neighborhood of zero. But in the limit $\epsilon \rightarrow 0$ with $\alpha$ held fixed, so that $\alpha / k$ is arbitrarily small, the only point in the moduli space $\mathcal{M}\left(\mathcal{K}_{\mathbf{p}, \mathbf{q}}\right)$ is the reducible point $\left\{\varrho_{\mathrm{ab}}\right\}$. Hence by analyticity, $Z\left(\epsilon ; \mathcal{K}_{\mathbf{p}, \mathbf{q}}, R\right)$ is completely determined under localization by the contribution from the single point $\left\{\varrho_{\mathrm{ab}}\right\} \cong \mathcal{O}_{\alpha} / G$.

We now turn to evaluating that contribution.

\subsection{Localization at the trivial connection on a Seifert homology sphere}

In this section, we perform our first localization computation for the Seifert loop operator. Throughout, we assume that $M$ is a Seifert homology sphere, or a cyclic $\mathbb{Z}_{d}$ quotient thereof. Our goal is to compute the local contribution to the Seifert loop path integral from the reducible point $\left\{\varrho_{\mathrm{ab}}\right\} \cong \mathcal{O}_{\alpha} / G$ in the Seifert loop moduli space $\mathcal{M}(C, \alpha)$.

From the perspective of (7.36), the point $\left\{\varrho_{\mathrm{ab}}\right\}$ can be considered to fiber equivariantly over the trivial connection on $M$. Essentially for this reason, the localization computation at $\left\{\varrho_{\mathrm{ab}}\right\}$ turns out to be a very natural extension of the computation in Section 5.2 of [18], where we evaluated the contribution from the trivial connection to the Chern-Simons partition function. Among the highlights of the present work, we will extract the renowned Weyl character formula from the Seifert loop path integral. Moreover, since we effectively treat the Seifert loop operator as a disorder operator, this computation provides a nice example of how, at least in principle, the expectation value of a disorder operator can be evaluated beyond the leading classical approximation.

\footnotetext{
${ }^{31}$ I thank E. Witten for remarks which substantially clarified the observations here.
} 


\subsubsection{The results of Lawrence and Rozansky revisited}

To prepare for our localization computation of the Seifert loop path integral, let us first discuss what results to expect based upon the exact expression for $Z(\epsilon ; C, R)$ as computed from conformal field theory. Once again, we rely on the work of Lawrence and Rozansky in [74], where the authors provide an exceedingly simple formula for $Z(\epsilon ; C, R)$ in the case that $C$ is the generic fiber of a Seifert homology sphere (or a cyclic $\mathbb{Z}_{d}$ quotient thereof) and the gauge group $G$ is $S U(2)$.

To express $Z(\epsilon ; C, R)$ in a manner which makes the semi-classical interpretation of the Seifert loop operator manifest, we find it useful to introduce the quantities

$$
\begin{aligned}
\epsilon_{r} & =\frac{2 \pi}{k+2} \\
\mathrm{P} & =\prod_{j=1}^{N} a_{j} \quad \text { if } N \geq 1, \quad \mathrm{P}=1 \quad \text { otherwise } \\
\theta_{0} & =3-\frac{d}{\mathrm{P}}+12 \sum_{j=1}^{N} s\left(b_{j}, a_{j}\right)
\end{aligned}
$$

Here $\epsilon_{r}$ is the renormalized coupling incorporating the famous shift $k \rightarrow k+2$ in the Chern-Simons level in the case $G=S U(2)$, and $s(b, a)$ is the Dedekind sum,

$$
s(b, a)=\frac{1}{4 a} \sum_{l=1}^{a-1} \cot \left(\frac{\pi l}{a}\right) \cot \left(\frac{\pi l b}{a}\right) .
$$

For sake of brevity, we also introduce the analytic functions

$$
\begin{aligned}
F(z) & =\left(2 \sinh \left(\frac{z}{2}\right)\right)^{2-N} \cdot \prod_{j=1}^{N}\left(2 \sinh \left(\frac{z}{2 a_{j}}\right)\right), \\
G^{(l)}(z) & =\frac{i}{4 \epsilon_{r}}\left(\frac{d}{\mathrm{P}}\right) z^{2}-\frac{2 \pi l}{\epsilon_{r}} z .
\end{aligned}
$$

Last but not least, we introduce the character $\operatorname{ch}_{\mathbf{j}}(z)$ for the irreducible representation $\mathbf{j}$ of $S U(2)$ with dimension $j$,

$$
\operatorname{ch}_{\mathbf{j}}(z)=\frac{\sinh (j z)}{\sinh (z)}=\mathrm{e}^{(j-1) z}+\mathrm{e}^{(j-3) z}+\cdots+\mathrm{e}^{-(j-3) z}+\mathrm{e}^{-(j-1) z} .
$$


According to the results of [74], the Seifert loop path integral on $M$ can then be written exactly as

$$
\begin{aligned}
Z(\epsilon ; C, \mathbf{j})= & (-1) \frac{\exp \left[\frac{3 \pi \mathrm{i}}{4}-\frac{\mathrm{i}}{4}\left(\theta_{0}+\left(j^{2}-1\right) \mathrm{P}\right) \epsilon_{r}\right]}{4 \sqrt{\mathrm{P}}} \\
& \times\left\{\sum_{l=0}^{d-1} \frac{1}{2 \pi i} \int_{\mathcal{C}^{(l)}} d z \operatorname{ch}_{\mathbf{j}}\left(\frac{z}{2}\right) F(z) \exp \left[G^{(l)}(z)\right]\right. \\
& -\left.\sum_{t=1}^{2 \mathrm{P}-1} \operatorname{Res}\left(\frac{\operatorname{ch}_{\mathbf{j}}\left(\frac{z}{2}\right) F(z) \exp \left[G^{(0)}(z)\right]}{1-\exp \left(-\frac{2 \pi}{\epsilon_{r}} z\right)}\right)\right|_{z=2 \pi i t} \\
& \left.-\left.\sum_{l=1}^{d-1} \sum_{t=1}^{\left[\frac{2 \mathrm{P} l}{d}\right]} \operatorname{Res}\left(\operatorname{ch}_{\mathbf{j}}\left(\frac{z}{2}\right) F(z) \exp \left[G^{(l)}(z)\right]\right)\right|_{z=-2 \pi i t}\right\} .
\end{aligned}
$$

Here, $\mathcal{C}^{(l)}$ for $l=0, \ldots, d-1$ denote a set of contours in the complex plane over which we evaluate the integrals in the first line of (7.62). In particular, $\mathcal{C}^{(0)}$ is the diagonal line contour through the origin,

$$
\mathcal{C}^{(0)}=\mathrm{e}^{\frac{\mathrm{i} \pi}{4}} \times \mathbb{R}
$$

and the other contours $\mathcal{C}^{(l)}$ for $l>0$ are diagonal line contours parallel to $\mathcal{C}^{(0)}$ running through the stationary phase point of the integrand, given by $z=-4 \pi \mathrm{i} l(\mathrm{P} / d)$. Also, "Res" denotes the residue of the given analytic function evaluated at the given point. Our formula for $Z(\epsilon ; C, \mathbf{j})$ in $(7.62)$ is a marginal extension of the corresponding formula in [74], where the authors focus attention on torus knots in $S^{3}$. However, (7.62) follows very easily from general results in [74]. A bit later, we will indeed specialize (7.62) to the particular case of torus knots.

We now wish to point out a few interesting features of (7.62) from the perspective of non-abelian localization.

First, if $\mathbf{j}=\mathbf{1}$ is the trivial representation, $\operatorname{ch}_{\mathbf{j}}(z)$ becomes the identity, and the formula for $Z(\epsilon ; C, \mathbf{j})$ in $(7.62)$ immediately reduces to a corresponding formula for the Chern-Simons partition function $Z(\epsilon)$ exhibited in Section 5.2 of [18]. In the case of the partition function, each summand in (7.62) is naturally identified with the contribution to $Z(\epsilon)$ from an associated component in the moduli space $\mathcal{M}$ of flat connections on $M$. Briefly, the $d$ integrals over the contours $\mathcal{C}^{(l)}$ appearing in the second line of (7.62) represent the local contributions to $Z(\epsilon)$ from the $d$ reducible flat connections on $M$, and the remaining residues in the final two lines of (7.62) can 
be identified with the contributions to $Z(\epsilon)$ from irreducible flat connections on $M$.

Second and perhaps more remarkably, we see from the formula in (7.62) that $Z(\epsilon ; C, \mathbf{j})$ has exactly the same structure as $Z(\epsilon)$ even when $\mathbf{j}$ is nontrivial. Again, $Z(\epsilon ; C, \mathbf{j})$ appears as a sum of terms associated to each component in the moduli space $\mathcal{M}$, and the Seifert loop operator is universally described on each component by the character $\mathrm{ch}_{\mathbf{j}}$. Of the terms in (7.62), the contour integral for $l=0$ represents the contribution from the trivial connection, which is given explicitly by

$$
\begin{aligned}
\left.Z(\epsilon ; C, \mathbf{j})\right|_{\{0\}}= & (-1) \frac{\exp \left[\frac{3 \pi \mathrm{i}}{4}-\frac{\mathrm{i}}{4}\left(\theta_{0}+\left(j^{2}-1\right) \mathrm{P}\right) \epsilon_{r}\right]}{4 \sqrt{\mathrm{P}}} \\
& \times \frac{1}{2 \pi \mathrm{i}} \int_{\mathcal{C}^{(0)}} d z \operatorname{ch}_{\mathbf{j}}\left(\frac{z}{2}\right) \exp \left[\frac{i}{4 \epsilon_{r}}\left(\frac{d}{\mathrm{P}}\right) z^{2}\right]\left(2 \sinh \left(\frac{z}{2}\right)\right)^{2-N} \\
& \times \prod_{j=1}^{N}\left(2 \sinh \left(\frac{z}{2 a_{j}}\right)\right)
\end{aligned}
$$

Of course, the Seifert loop path integral does not localize on the moduli space $\mathcal{M}$ but on its extended cousin $\mathcal{N}(C, \alpha)$, so the apparent structure in (7.62) deserves further explanation. Although the formula (7.62) for $Z(\epsilon ; C, \mathbf{j})$ is valid for arbitrary values of $\epsilon$ and $\mathbf{j}$, its semi-classical interpretation holds in the particular regime $\epsilon \rightarrow 0$ with $\mathbf{j}$ held fixed. In that regime, the parameter $\alpha / k$ is small, and each component of $\mathcal{M}(C, \alpha)$ fibers over a corresponding component of $\mathcal{N}$, with fiber $\mathcal{O}_{\alpha}$. Pushing down over $\mathcal{O}_{\alpha}$, we then identify the contributions from such fibered components of $\mathcal{M}(C, \alpha)$ with contributions from $\mathcal{M}$, just as in (7.62). Indeed, the major result in this section is to reproduce the particular term in (7.64) by localization at the distinguished reducible point $\left\{\varrho_{\mathrm{ab}}\right\} \cong \mathcal{O}_{\alpha} / G$, which fibers over the trivial connection $\{0\}$ on the Seifert homology sphere $M$.

The preceding limit for the parameters $\epsilon$ and $\mathbf{j}$ should be contrasted with the alternative semi-classical limit in which $\epsilon$ is taken to zero with the product $\epsilon \mathbf{j}$ held fixed. Equivalently, the Chern-Simons level $k$ goes to infinity with $\alpha / k$ non-zero and finite. In the latter limit, relevant for the generalized volume conjecture [86], the moduli space $\mathcal{M}(C, \alpha)$ can have components which do not fiber over components of $\mathcal{M}$, as we exhibited explicitly for torus knots $\mathcal{K}_{\mathbf{p}, \mathbf{q}} \subset S^{3}$ at the end of Section 7.1. The contributions to $Z(\epsilon ; C, \mathbf{j})$ from the additional, non-fibered components in $\mathcal{M}(C, \alpha)$ then become visible only when the exact expression in (7.62) is rewritten in an asymptotic form compatible with the stationary-phase approximation at finite (non-zero) values for $\alpha / k$. See for example the "Main Theorem" in [45], where such an asymptotic expansion is carried out for torus knots in $S^{3}$. 
Finally, the phase of $Z(\epsilon ; C, \mathbf{j})$ in (7.62) is quite subtle. This phase depends upon both a two-framing of the manifold $M$ as well as a framing of the curve $C$. By definition, a two-framing of $M$ is a trivialization of the direct sum $T M \oplus T M$ of two copies of the tangent bundle $T M$. As explained in [13], a canonical choice (up to homotopy) of two-framing exists for each threemanifold.

Similarly, a framing of the curve $C$ is specified by non-vanishing normal vector field on $C$. Such a vector field determines a small displacement $C^{\prime}$ of $C$ inside $M$, as would be used for instance in a point-splitting regularization of the Wilson loop operator. If $M$ is a homology sphere, Alexander duality (see for instance Theorem 3.44 in [71]) implies that $H_{1}\left(M^{o} ; \mathbb{Z}\right) \cong \mathbb{Z}$ is freely generated by the meridian $\mathrm{m}$ of $C$. In this case, $C$ also carries a canonical framing, determined by the condition that $C^{\prime}$ and $C$ have zero linking number inside $M$, where the linking number is defined by $\operatorname{lk}\left(C^{\prime}, C\right)=\left[C^{\prime}\right]$ in $H_{1}\left(M^{o} ; \mathbb{Z}\right)$.

Since $M$ carries a canonical two-framing and $C$ carries a canonical framing, $Z(\epsilon ; C, \mathbf{j})$ can be presented with a definite phase, as given in (7.62). The phase of $Z(\epsilon ; C, \mathbf{j})$ that arises naturally when we define Chern-Simons theory via localization actually differs from the canonical phase, and we discuss this fact at the end of the section.

\subsubsection{Special case: the Jones polynomial of a torus knot}

To gain a bit more insight into the empirical formula (7.62) for $Z(\epsilon ; C, \mathbf{j})$, let us again specialize to the case of torus knots $\mathcal{K}_{\mathbf{p}, \mathbf{q}}$ in $S^{3}$.

With the Seifert invariants given in (7.39) and (7.42), the formula for $Z(\epsilon ; C, \mathbf{j})$ becomes

$$
\begin{aligned}
Z(\epsilon ; & \left.\mathcal{K}_{\mathbf{p}, \mathbf{q}}, \mathbf{j}\right) \\
= & (-1) \frac{\exp \left[\frac{3 \pi \mathrm{i}}{4}-\frac{\mathrm{i}}{4}\left(\frac{\mathbf{p}}{\mathbf{q}}+\frac{\mathbf{q}}{\mathbf{p}}+\mathbf{p q}\left(j^{2}-1\right)\right) \epsilon_{r}\right]}{\sqrt{\mathbf{p q}}} \\
& \times\left\{\frac{1}{2 \pi \mathrm{i}} \int_{\mathcal{C}^{(0)}} d z \operatorname{ch}_{\mathbf{j}}\left(\frac{z}{2}\right) \sinh \left(\frac{z}{2 \mathbf{p}}\right) \sinh \left(\frac{z}{2 \mathbf{q}}\right) \exp \left[\frac{\mathrm{i}}{4 \epsilon_{r}}\left(\frac{1}{\mathbf{p q}}\right) z^{2}\right]\right. \\
& \left.+\left(\frac{j}{k+2}\right) \sum_{t=1}^{2 \mathbf{p q}-1}(-1)^{t(j+1)} \sin \left(\frac{\pi t}{\mathbf{p}}\right) \sin \left(\frac{\pi t}{\mathbf{q}}\right) \exp \left(\frac{-\mathrm{i} \pi(k+2)}{2 \mathbf{p q}} t^{2}\right)\right\} .
\end{aligned}
$$

In passing from (7.62) to (7.65), we have explicitly evaluated the phase $\theta_{0}$ in (7.58) for the Seifert presentation of $S^{3}$ with fiber $\mathcal{K}_{\mathbf{p}, \mathbf{q}}$. Here we use 
two arithmetic properties of the Dedekind sum $s(\cdot, \cdot)$ that enters $\theta_{0}$. First, as follows more or less directly from the definition in (7.59),

$$
s(\mathbf{p}-\mathbf{r}, \mathbf{p})=s(\mathbf{q}, \mathbf{p}), \quad s(\mathbf{s}, \mathbf{q})=s(\mathbf{p}, \mathbf{q}), \quad \mathbf{p s}-\mathbf{q r}=1 .
$$

Much more non-trivially, we also use Dedekind reciprocity, which states that

$$
12 \mathbf{p q}[s(\mathbf{p}, \mathbf{q})+s(\mathbf{q}, \mathbf{p})]=\mathbf{p}^{2}+\mathbf{q}^{2}-3 \mathbf{p q}+1, \quad \operatorname{gcd}(\mathbf{p}, \mathbf{q})=1 .
$$

See Section 3.8 of [6] for an elementary (but by no means obvious) proof of Dedekind reciprocity. Together, we apply (7.66) and (7.67) to compute $\theta_{0}$ as

$$
\theta_{0}=3-\frac{1}{\mathbf{p q}}+12[s(\mathbf{p}-\mathbf{r}, \mathbf{p})+s(\mathbf{s}, \mathbf{q})]=\frac{\mathbf{p}}{\mathbf{q}}+\frac{\mathbf{q}}{\mathbf{p}} .
$$

We have also evaluated the residues appearing in the empirical formula for $Z(\epsilon ; C, R)$. These residues appear in the sum over $t$ in $(7.65)$, in terms of which we decompose $Z\left(\epsilon ; \mathcal{K}_{\mathbf{p}, \mathbf{q}}, \mathbf{j}\right)$ as

$$
Z\left(\epsilon ; \mathcal{K}_{\mathbf{p}, \mathbf{q}}, \mathbf{j}\right)=\left.Z\left(\epsilon ; \mathcal{K}_{\mathbf{p}, \mathbf{q}}, \mathbf{j}\right)\right|_{\{0\}}+Z\left(\epsilon ; \mathcal{K}_{\mathbf{p}, \mathbf{q}}, \mathbf{j}\right)_{\text {res }},
$$

where

$$
\begin{aligned}
Z(\epsilon ; & \left.\mathcal{K}_{\mathbf{p}, \mathbf{q}}, \mathbf{j}\right)\left.\right|_{\{0\}} \\
= & (-1) \frac{\exp \left[\frac{3 \pi \mathrm{i}}{4}-\frac{\mathrm{i}}{4}\left(\frac{\mathbf{p}}{\mathbf{q}}+\frac{\mathbf{q}}{\mathbf{p}}+\mathbf{p q}\left(j^{2}-1\right)\right) \epsilon_{r}\right]}{\sqrt{\mathbf{p q}}} \\
& \times \frac{1}{2 \pi \mathrm{i}} \int_{\mathcal{C}^{(0)}} d z \operatorname{ch}_{\mathbf{j}}\left(\frac{z}{2}\right) \sinh \left(\frac{z}{2 \mathbf{p}}\right) \sinh \left(\frac{z}{2 \mathbf{q}}\right) \exp \left[\frac{\mathrm{i}}{4 \epsilon_{r}}\left(\frac{1}{\mathbf{p q}}\right) z^{2}\right],
\end{aligned}
$$

and

$$
\begin{aligned}
Z(\epsilon ; & \left.\mathcal{K}_{\mathbf{p}, \mathbf{q}}, \mathbf{j}\right)_{\text {res }} \\
= & (-1) \frac{\exp \left[\frac{3 \pi \mathrm{i}}{4}-\frac{\mathrm{i}}{4}\left(\frac{\mathbf{p}}{\mathbf{q}}+\frac{\mathbf{q}}{\mathbf{p}}+\mathbf{p q}\left(j^{2}-1\right)\right) \epsilon_{r}\right]}{\sqrt{\mathbf{p q}}} \\
& \times\left(\frac{j}{k+2}\right) \sum_{t=1}^{2 \mathbf{p q}-1}(-1)^{t(j+1)} \sin \left(\frac{\pi t}{\mathbf{p}}\right) \sin \left(\frac{\pi t}{\mathbf{q}}\right) \exp \left(\frac{-\mathrm{i} \pi(k+2)}{2 \mathbf{p q}} t^{2}\right) .
\end{aligned}
$$


As we have already mentioned, $\left.Z\left(\epsilon ; \mathcal{K}_{\mathbf{p}, \mathbf{q}}, \mathbf{j}\right)\right|_{\{0\}}$ can be naturally interpreted as the contribution to the Seifert loop path integral from the reducible point $\left\{\varrho_{\mathrm{ab}}\right\}$ in the extended moduli space $\mathcal{M}\left(\mathcal{K}_{\mathbf{p}, \mathbf{q}}, \mathbf{j}\right)$. Equivalently, via equivariant pushdown in the fibration $(7.36),\left.Z\left(\epsilon ; \mathcal{K}_{\mathbf{p}, \mathbf{q}}, \mathbf{j}\right)\right|_{\{0\}}$ is the contribution from the trivial connection $\{0\}$ on $S^{3}$. But in the relevant semiclassical limit, for which $\epsilon \rightarrow 0$ with $\mathbf{j}$ fixed, $\left\{\varrho_{\mathrm{ab}}\right\}$ is indeed the only point in $\mathcal{N}\left(\mathcal{K}_{\mathbf{p}, \mathbf{q}}, \mathbf{j}\right)$. Hence, our localization result for the Seifert loop path integral implies that the additional, oscillatory Gaussian sum in (7.71) must actually vanish,

$$
Z\left(\epsilon ; \mathcal{K}_{\mathbf{p}, \mathbf{q}}, \mathbf{j}\right)_{\text {res }}=0, \quad \operatorname{gcd}(\mathbf{p}, \mathbf{q})=1
$$

As a small check, we verify this statement directly in Appendix B.

After applying the vanishing result (7.72) from Appendix B, we obtain a wonderfully compact formula ${ }^{32}$ for the expectation value of an arbitrary Wilson loop operator wrapping the torus knot $\mathcal{K}_{\mathbf{p}, \mathbf{q}}$ in $S^{3}$ and decorated with the irreducible $S U(2)$ representation $\mathbf{j}$,

$$
\begin{aligned}
Z\left(\epsilon ; \mathcal{K}_{\mathbf{p}, \mathbf{q}}, \mathbf{j}\right)= & \left.Z\left(\epsilon ; \mathcal{K}_{\mathbf{p}, \mathbf{q}}, \mathbf{j}\right)\right|_{\{0\}}, \\
= & \frac{1}{2 \pi \mathrm{i}} \frac{1}{\sqrt{\mathbf{p q}}} \exp \left[-\frac{\mathrm{i} \pi}{2(k+2)}\left(\frac{\mathbf{p}}{\mathbf{q}}+\frac{\mathbf{q}}{\mathbf{p}}+\mathbf{p q}\left(j^{2}-1\right)\right)\right] \\
& \times \int_{\mathbb{R}} d x \operatorname{ch}_{\mathbf{j}}\left(\mathrm{e}^{\frac{\mathrm{i} \pi}{4}} \frac{x}{2}\right) \sinh \left(\mathrm{e}^{\frac{\mathrm{i} \pi}{4}} \frac{x}{2 \mathbf{p}}\right) \sinh \left(\mathrm{e}^{\frac{\mathrm{i} \pi}{4}} \frac{x}{2 \mathbf{q}}\right) \\
& \times \exp \left[-\frac{(k+2)}{8 \pi}\left(\frac{x^{2}}{\mathbf{p q}}\right)\right] .
\end{aligned}
$$

In writing (7.73), we have rotated the contour $\mathcal{C}^{(0)}=\mathrm{e}^{\mathrm{i} \pi} \frac{\mathrm{i}}{4} \times \mathbb{R}$ to the real axis and substituted $\epsilon_{r}=2 \pi /(k+2)$, so that (7.73) appears as a simple generalization of the corresponding formula (1.8) for the unknot $\bigcirc=\mathcal{K}_{1,1}$.

The expression for $Z\left(\epsilon ; \mathcal{K}_{\mathbf{p}, \mathbf{q}}, \mathbf{j}\right)$ in (7.73) has several remarkable properties, which also provide an independent check that (7.73) is correct. First, if $\mathbf{j}=\mathbf{1}$, then all dependence on $\mathbf{p}$ and $\mathbf{q}$ cancels between the prefactor and the integral over $x$ in $(7.73)$, and our formula for $Z\left(\epsilon ; \mathcal{K}_{\mathbf{p}, \mathbf{q}}, \mathbf{j}\right)$ reproduces

\footnotetext{
${ }^{32} \mathrm{~A}$ related formula appears in Lemma 1 of [76].
} 
the standard result for the $S U(2)$ Chern-Simons partition function on $S^{3}$,

$$
Z(k)=\sqrt{\frac{2}{k+2}} \sin \left(\frac{\pi}{k+2}\right) .
$$

Second, if either $\mathbf{p}, \mathbf{q}=1$ and the other is an arbitrary non-zero integer, then $\mathcal{K}_{\mathbf{p}, \mathbf{q}}$ is equivalent to the unknot. As one can verify, (7.73) still reproduces the expected result for $Z(k ; \bigcirc, \mathbf{j})$ in that more general case,

$$
Z(k ; \bigcirc, \mathbf{j})=\sqrt{\frac{2}{k+2}} \sin \left(\frac{\pi j}{k+2}\right) .
$$

Finally, the integral over $x$ in (7.73) is just a sum of Gaussian integrals, which can be evaluated explicitly as

$$
\begin{aligned}
Z\left(\epsilon ; \mathcal{K}_{\mathbf{p}, \mathbf{q}}, \mathbf{j}\right)= & \frac{1}{2 \mathrm{i}} \sqrt{\frac{2}{k+2}} \mathrm{t}^{\frac{1}{4} \mathbf{p q}\left(j^{2}-1\right)} \sum_{l=0}^{j-1} \mathrm{t}^{-\frac{1}{4} l_{*}^{2} \mathbf{p q}-\frac{1}{2} l_{*} \mathbf{p}} \\
& \times\left[\mathrm{t}^{-\frac{1}{2}\left(l_{*} \mathbf{q}+1\right)}-\mathrm{t}^{\frac{1}{2}\left(l_{*} \mathbf{q}+1\right)}\right] \\
l_{*}= & 2 l-j+1, \quad \mathrm{t}=\exp \left(-\frac{2 \pi \mathrm{i}}{k+2}\right)
\end{aligned}
$$

The sum over $l$ in (7.76) arises from the sum (7.61) over exponentials in the character $\mathrm{ch}_{\mathbf{j}}$, and we have introduced the standard variable $t$ that appears in the Jones polynomial.

When $\mathbf{j}=\mathbf{2}$, the sum in (7.76) must reproduce the Jones polynomial of the torus knot $\mathcal{K}_{\mathbf{p}, \mathbf{q}}$. Concretely,

$$
Z\left(\epsilon ; \mathcal{K}_{\mathbf{p}, \mathbf{q}}, \mathbf{2}\right)=\frac{1}{2 i} \sqrt{\frac{2}{k+2}} \mathrm{t}^{\frac{1}{2}(\mathbf{p q}-\mathbf{p}-\mathbf{q}-1)}\left[1+\mathrm{t}^{\mathbf{p}+\mathbf{q}}-\mathrm{t}^{\mathbf{p}+1}-\mathrm{t}^{\mathbf{q}+1}\right] .
$$

The Jones polynomial $V_{\mathcal{K}}(\mathrm{t})$ for a knot $\mathcal{K}$ is generally proportional to $Z(\epsilon ; \mathcal{K}, \mathbf{2})$. However, $V_{\mathcal{K}}(\mathrm{t})$ is conventionally normalized so that for the unknot, $V_{\bigcirc}(\mathrm{t})=1$. Rescaling $(7.77)$ by $Z\left(\epsilon ; \mathcal{K}_{\mathbf{1}, \mathbf{1}}, \mathbf{2}\right)$, we find

$$
V_{\mathcal{K}_{\mathbf{p}, \mathbf{q}}}(\mathrm{t})=\frac{Z\left(\epsilon ; \mathcal{K}_{\mathbf{p}, \mathbf{q}}, \mathbf{2}\right)}{Z\left(\epsilon ; \mathcal{K}_{\mathbf{1}, \mathbf{1}}, \mathbf{2}\right)}=\frac{\mathrm{t}^{\frac{1}{2}(\mathbf{p}-1)(\mathbf{q}-1)}}{1-\mathrm{t}^{2}}\left[1+\mathrm{t}^{\mathbf{p}+\mathbf{q}}-\mathrm{t}^{\mathbf{p}+1}-\mathrm{t}^{\mathbf{q}+1}\right] .
$$

This formula for the Jones polynomial of a torus knot is a classic result that goes back to Jones himself [68], and early after Witten's foundational work [105], the same result was obtained in $[73,80]$ using the Hamiltonian 
formulation of Chern-Simons theory. Among the many motivations for this paper, one is to explain how $V_{\mathcal{K}_{\mathbf{p}, \mathbf{q}}}(\mathrm{t})$ can be alternatively computed using non-abelian localization, as we now discuss.

\subsubsection{A symplectic model for $\mathcal{O}_{\alpha} \subset \overline{\mathcal{A}}_{\alpha}$}

In the remainder of this section, we wish to apply the non-abelian localization formula in (6.31) to compute directly the contribution from the reducible point $\left\{\varrho_{\mathrm{ab}}\right\} \cong \mathcal{O}_{\alpha} / G$ in $\mathcal{M}(C, \alpha)$ to the Seifert loop path integral. Though we presented the corresponding empirical result (7.64) of Lawrence and Rozansky for the special case $G=S U(2)$, no extra effort is required to treat the case of an arbitrary compact, connected, simply connected, and simple Lie group $G$, as we shall do here. See also [81] for work extending the results of [74] to arbitrary $G$.

Of course, in order to apply the non-abelian localization formula to our problem, we first need to determine the local symplectic geometry in an equivariant neighborhood of the orbit $\mathcal{O}_{\alpha}$ embedded as a finite-dimensional submanifold of the infinite-dimensional product $\overline{\mathcal{A}}_{\alpha}=\overline{\mathcal{A}} \times \epsilon L \mathcal{O}_{\alpha}$,

$$
\mathcal{O}_{\alpha} \subset \overline{\mathcal{A}}_{\alpha}=\overline{\mathcal{A}} \times \epsilon L \mathcal{O}_{\alpha} .
$$

Following the ansatz in Section 6.2, we model an equivariant neighborhood $N$ of $\mathcal{O}_{\alpha} \subset \overline{\mathcal{A}}_{\alpha}$ on a symplectic fibration of the general form

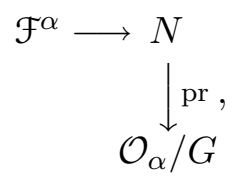

where the fiber $\mathcal{F}^{\alpha}$ is itself the total space of a homogeneous vector bundle over a quotient $\mathcal{H} / H_{0}^{\alpha}$. Specifically, in complete correspondence to (6.21),

$$
\mathcal{F}^{\alpha}=\mathcal{H} \times_{H_{0}^{\alpha}}\left(\mathfrak{h}^{\perp} \oplus \mathcal{E}_{1}^{\alpha}\right), \quad \mathfrak{h}^{\perp} \equiv \mathfrak{h} \ominus \mathfrak{h}_{0}^{\alpha} \ominus \mathcal{E}_{0}^{\alpha} .
$$

Here $H_{0}^{\alpha}$ is the subgroup of the Hamiltonian group $\mathcal{H}$ which preserves points in $\mathcal{O}_{\alpha}$, and we recall from Section 4.3 that we have already identified $\mathcal{H}$ to be

$$
\mathcal{H}=U(1)_{\mathrm{R}} \ltimes \widetilde{\mathcal{G}_{0}} .
$$

So to specify the geometry of $\mathcal{F}^{\alpha}$ in (7.81), we are left to determine $H_{0}^{\alpha}$ as well as the vector spaces $\left(\mathcal{E}_{0}^{\alpha}, \mathcal{E}_{1}^{\alpha}\right)$. 
The local model for $\mathcal{F}^{\alpha}$, which describes a neighborhood of $\mathcal{O}_{\alpha}$ in $\overline{\mathcal{A}}_{\alpha}$ turns out to be a natural generalization of the model we applied in Section 5.2 of [18] to describe a neighborhood of the trivial connection $\{0\}$ in $\overline{\mathcal{A}}$. Both to compare with our previous work and to economize the present discussion, let us therefore quickly recall the analogous data $\left(H_{0}, \mathcal{E}_{0}, \mathcal{E}_{1}\right)$ which specify the local model describing a neighborhood of $\{0\} \in \overline{\mathcal{A}}$. With these data in hand, the extension to $\left(H_{0}^{\alpha}, \mathcal{E}_{1}^{\alpha}, \mathcal{E}_{a}^{\alpha}\right)$ will be more or less straightforward.

First, under the action of the Hamiltonian group $\mathcal{H}$, the trivial connection on $M$ is fixed by the subgroup

$$
H_{0}=U(1)_{\mathrm{R}} \times G \times U(1)_{\mathrm{z}}
$$

where $G$ is identified with the group of constant gauge transformations on $M$. To play the roles of $E_{0}$ and $E_{1}$ in the abstract symplectic model of Section 6.2 , we also introduce vector spaces $\left(\mathcal{E}_{0}, \mathcal{E}_{1}\right)$. Both $\mathcal{E}_{0}$ and $\mathcal{E}_{1}$ turn out to be quite delicate to determine, and we refer the reader to Section 5.1 of [18] for a complete discussion. Suffice it to say, $\mathcal{E}_{0}$ and $\mathcal{E}_{1}$ are given by certain direct sums of Dolbeault cohomology groups,

$$
\begin{aligned}
& \mathcal{E}_{0}=\bigoplus_{t \geq 1} H \frac{0}{\partial}\left(\Sigma, \mathfrak{g} \otimes\left(\mathcal{L}^{t} \oplus \mathcal{L}^{-t}\right)\right), \\
& \mathcal{E}_{1}=\bigoplus_{t \geq 1} H \frac{1}{\partial}\left(\Sigma, \mathfrak{g} \otimes\left(\mathcal{L}^{t} \oplus \mathcal{L}^{-t}\right)\right) .
\end{aligned}
$$

Here $\Sigma$ is the Riemann surface (or more generally orbifold) sitting at the base of $M$, and $\mathcal{L}$ is the line bundle over $\Sigma$ associated to the Seifert presentation of $M$. In general, both $H_{\frac{0}{\partial}}\left(\Sigma, \mathfrak{g} \otimes\left(\mathcal{L}^{t} \oplus \mathcal{L}^{-t}\right)\right)$ and $H_{\bar{\partial}}\left(\Sigma, \mathfrak{g} \otimes\left(\mathcal{L}^{t} \oplus\right.\right.$ $\left.\mathcal{L}^{-t}\right)$ ) are non-vanishing for arbitrarily large $t$, so $\mathcal{E}_{0}$ and $\mathcal{E}_{1}$ have infinite dimension.

To describe a neighborhood of $\mathcal{O}_{\alpha} \subset \overline{\mathcal{A}}_{\alpha}$, we must modify both $H_{0}$ in (7.83) and $\left(\mathcal{E}_{0}, \mathcal{E}_{1}\right)$ in $(7.84)$. Clearly, the stabilizer $H_{0}^{\alpha}$ of a point in $\mathcal{O}_{\alpha}$ will only be a subgroup of the stabilizer $H_{0}$ for the trivial connection. As we noted following (7.34), points in $\mathcal{O}_{\alpha}$ are represented by reducible connections which are preserved by constant gauge transformations in $G_{\alpha} \subset G$. These connections are also invariant under the $U(1)_{\mathrm{R}}$ action on $M$, so immediately

$$
H_{0}^{\alpha}=U(1)_{\mathrm{R}} \times G_{\alpha} \times U(1)_{\mathrm{Z}}
$$

By way of illustration, we recall that if $\alpha$ is regular, then $G_{\alpha}=T$ is a maximal torus of $G$. At the other extreme, if $\alpha=0$, then $G_{\alpha}=G$ and $H_{0}^{\alpha}$ reduces to $H_{0}$. 
"ATMP-17-1-A1-BEA" — 2013/5/15 — 19:32 — page 146 — \#146

We are left to specify the vector spaces $\left(\mathcal{E}_{0}^{\alpha}, \mathcal{E}_{1}^{\alpha}\right)$ in (7.81). By construction, the vector spaces $\left(\mathcal{E}_{0}, \mathcal{E}_{1}\right)$ in (7.84) already provide a local model for the normal directions to $\mathcal{O}_{\alpha} \subset \overline{\mathcal{A}}_{\alpha}=\overline{\mathcal{A}} \times \epsilon L \mathcal{O}_{\alpha}$ which lie along $\overline{\mathcal{A}}$. To account further for the symplectic geometry of the loopspace $L \mathcal{O}_{\alpha}$, we need only enlarge $\mathcal{E}_{0}$ and $\mathcal{E}_{1}$ slightly. We discuss the required modifications in turn.

As we explained in Section 4.3 of [18] and briefly indicated in Section 6.2 , the role of $E_{0}$ in the abstract model for $F$ is to control the symplectic structure on a neighborhood of $H / H_{0} \subseteq F$. In essence, the possible symplectic models for the embedding of $H / H_{0}$ inside $F$ interpolate from a cotangent model, for which $H / H_{0}$ is embedded as the zero section of the cotangent bundle $T^{*}\left(H / H_{0}\right)$, to a coadjoint model, for which $H / H_{0}$ carries the coadjoint symplectic form and no cotangent fibers are present in $F$. In the cotangent model, $E_{0}$ is trivial, and in the coadjoint model, $E_{0}$ coincides with the holomorphic tangent space to $H / H_{0}$ at the identity, as we illustrated in (6.47).

In the case at hand, since $\mathcal{H}$ contains a copy of $H_{0}$, the homogeneous space $\mathcal{H} / H_{0}^{\alpha}$ sitting at the base of $\mathcal{F}^{\alpha}$ contains a copy of

$$
H_{0} / H_{0}^{\alpha}=G / G_{\alpha}=\mathcal{O}_{\alpha}
$$

So we see that $\mathcal{H} / H_{0}^{\alpha}$ already contains a copy of the orbit $\mathcal{O}_{\alpha}$ on which we localize. However, we also know that $\mathcal{O}_{\alpha}$ must carry the canonical coadjoint symplectic form. According to the abstract symplectic model sketched above, if $\mathcal{O}_{\alpha} \subset \mathcal{H} / H_{0}^{\alpha}$ is to carry the coadjoint symplectic form, the vector space $\mathcal{E}_{0}^{\alpha}$, which enters (7.81) must include the holomorphic tangent space $\mathfrak{g}^{(1,0)}$ to $\mathcal{O}_{\alpha}$ as a summand.

Otherwise, the original vector space $\mathcal{E}_{0}$ in (7.84) already encodes the correct symplectic form on the remaining directions normal to $\mathcal{O}_{\alpha}$ inside $\mathcal{H} / H_{0}^{\alpha}$. Hence the new vector space $\mathcal{E}_{0}^{\alpha}$ is given merely by the direct sum

$$
\mathcal{E}_{0}^{\alpha}=\mathcal{E}_{0} \oplus \mathfrak{g}^{(1,0)}
$$

For future reference, we recall from $(4.23)$ that $\mathfrak{g}^{(1,0)}$ is given concretely by the following sum of rootspaces $\mathfrak{e}_{\beta} \subset \mathfrak{g}_{\mathbb{C}}$,

$$
\mathfrak{g}^{(1,0)}=\bigoplus_{(\beta, \alpha)>0} \mathfrak{e}_{\beta}, \quad \beta \in \mathfrak{R}
$$

When $\alpha$ is regular, $\mathfrak{g}^{(1,0)}=\mathfrak{g}_{+}$is the entire positive rootspace. 
Having identified the analogue of $E_{0}$ in the abstract symplectic model, we now consider $E_{1}$. The role of $E_{1}$ in the symplectic model for $F$ is to describe whatever directions are normal to $H / H_{0}$ beyond those already captured in the interpolating fiber $\mathfrak{h}^{\perp}=\mathfrak{h} \ominus \mathfrak{h}_{0} \ominus E_{0}$. In the case at hand, the vector space $\mathcal{E}_{1}$ in (7.84) already encodes such directions normal to $\mathcal{O}_{\alpha} \subset \overline{\mathcal{A}} \times \epsilon L \mathcal{O}_{\alpha}$ inside $\overline{\mathcal{A}}$. So, we are left to consider the other directions normal to $\mathcal{O}_{\alpha}$ inside the loopspace $L \mathcal{O}_{\alpha}$.

In general, the tangent space at a point $[U] \in L \mathcal{O}_{\alpha}$ corresponding to a given map $U: C \rightarrow \mathcal{O}_{\alpha}$ is the space of sections of the pullback $U^{*}\left(T \mathcal{O}_{\alpha}\right)$. Localizing on $\mathcal{O}_{\alpha}$, we are interested in the special case that $U(\tau)=U_{0}$ is constant. In that case, $U^{*}\left(T \mathcal{O}_{\alpha}\right)$ can be identified up to the action of $G$ as the trivial bundle on $C$ with fiber $\mathfrak{g} \ominus \mathfrak{g}_{\alpha}$. The tangent space to $L \mathcal{O}_{\alpha}$ at a point in $\mathcal{O}_{\alpha}$ is then isomorphic to the space of maps $\delta U: C \rightarrow \mathfrak{g} \ominus \mathfrak{g}_{\alpha}$.

By assumption, $C$ is a Seifert fiber of $M$ and hence is preserved under the action of $U(1)_{R}$. We can thus decompose the map $\delta U$ into eigenmodes of the Lie derivative $£_{R}$ along the vector field $R$,

$$
\delta U=\sum_{t=-\infty}^{+\infty} \delta U_{t},
$$

such that each eigenmode $\delta U_{t}$ satisfies

$$
£_{\mathrm{R}} \delta U_{t}=-2 \pi \mathrm{i} t \cdot \delta U_{t} .
$$

Of course, (7.89) is nothing more than an infinitesimal version of the decomposition of $U$ into Fourier modes along $C$.

The constant eigenmode $\delta U_{0}$ describes tangent directions to $\mathcal{O}_{\alpha}$ itself, and we have already accounted for these directions with the summand $\mathfrak{g}^{(1,0)} \subset \mathcal{E}_{0}^{\alpha}$. In contrast, the eigenmodes $\delta U_{t}$ with $t \neq 0$ describe non-trivial normal directions to $\mathcal{O}_{\alpha}$ inside $L \mathcal{O}_{\alpha}$. Thus, the normal fiber $\mathcal{N}_{\alpha}$ to $\mathcal{O}_{\alpha}$ embedded in $L \mathcal{O}_{\alpha}$ consists of a countable sum of copies of $\mathfrak{g} \ominus \mathfrak{g}_{\alpha}$, graded by the non-zero integer $t$.

Of course, $\mathfrak{g} \ominus \mathfrak{g}_{\alpha}$ is a real vector space. As we discussed in Section 4.3, the loopspace $L \mathcal{O}_{\alpha}$ carries a complex structure induced pointwise from the complex structure on $\mathcal{O}_{\alpha}$. So as a complex vector space,

$$
\mathcal{N}_{\alpha}=\bigoplus_{t \geq 1}\left[\mathfrak{g}_{t}^{(1,0)} \oplus \mathfrak{g}_{-t}^{(1,0)}\right] .
$$


Here, the subscript on each copy of $\mathfrak{g}^{(1,0)}$ indicates the Fourier eigenvalue in (7.90).

Since the role of $\mathcal{E}_{1}^{\alpha}$ is to account for directions normal to $\mathcal{O}_{\alpha}$ in both $\overline{\mathcal{A}}$ and $L \mathcal{O}_{\alpha}$, one might guess that $\mathcal{E}_{1}^{\alpha}$ is given by the direct sum $\mathcal{E}_{1} \oplus \mathcal{N}_{\alpha}$. To the first approximation this guess is correct, but we must be careful about the choice of complex structure on $\mathcal{E}_{1}^{\alpha}$.

According to the convention in (6.23), the complex structure on the abstract vector space $E_{1}$ in the non-abelian localization formula (6.31) is defined so that $-\mathrm{i} \gamma_{0}$ acts with strictly negative eigenvalues on a holomorphic basis of $E_{1}$. This convention is opposite to the convention in (4.23), which defines the complex structure on $\mathcal{O}_{\alpha}$, since $\mathfrak{g}^{(1,0)}$ is defined to be the positive eigenspace of $-\mathrm{i} \alpha$ under the adjoint action. To account for the relative sign in our two conventions, we define $\mathcal{E}_{1}^{\alpha}$ using not $\mathcal{N}_{\alpha}$ in (7.91) but rather the conjugate vector space $\overline{\mathcal{N}}_{\alpha}$,

$$
\overline{\mathcal{N}}_{\alpha}=\bigoplus_{t \geq 1}\left[\mathfrak{g}_{t}^{(0,1)} \oplus \mathfrak{g}_{-t}^{(0,1)}\right]
$$

Here, $\mathfrak{g}^{(0,1)}$ is the anti-holomorphic tangent space to $\mathcal{O}_{\alpha}$, which is spanned by the rootspaces $\mathfrak{e}_{-\beta}$ associated to negative roots $-\beta<0$ of $G$,

$$
\mathfrak{g}^{(0,1)}=\bigoplus_{(\beta, \alpha)>0} \mathfrak{e}_{-\beta}, \quad \beta \in \mathfrak{R}
$$

So if we are careful about the complex structure,

$$
\mathcal{E}_{1}^{\alpha}=\mathcal{E}_{1} \oplus \overline{\mathcal{N}}_{\alpha}
$$

As will be clear in our later computations, the appearance of $\overline{\mathcal{N}}_{\alpha}$ as opposed to $\mathcal{N}_{\alpha}$ in (7.94) is actually crucial for the interpretation of the Seifert loop operator as the character associated to the representation $R$.

Altogether, (7.85), (7.87), and (7.94) specify the local symplectic model for the embedding $\mathcal{O}_{\alpha} \subset \overline{\mathcal{A}}_{\alpha}$.

\subsubsection{Non-abelian localization on $\mathcal{O}_{\alpha} / G$}

With the symplectic model for $\mathcal{F}^{\alpha}$ in hand, we now apply the non-abelian localization formula in (6.31) to the Seifert loop path integral. Since $\left\{\varrho_{a b}\right\} \cong$ $\mathcal{O}_{\alpha} / G$ is a point, the path integral immediately reduces via (6.41) to an 
integral over the finite-dimensional Lie algebra $\mathfrak{h}_{0}^{\alpha}=\mathbb{R} \oplus \mathfrak{g}_{\alpha} \oplus \mathbb{R}$ of the stabilizer $H_{0}^{\alpha}$,

$$
\begin{aligned}
\left.Z(\epsilon ; C, R)\right|_{\mathcal{O}_{\alpha} / G}= & \frac{(2 \pi \epsilon)}{\operatorname{Vol}\left(G_{\alpha}\right)} \int_{\mathfrak{h}_{0}^{\alpha}}\left[\frac{d \psi}{2 \pi}\right] \operatorname{det}\left(\left.\frac{\psi}{2 \pi}\right|_{\mathcal{E}_{0}^{\alpha}}\right) \operatorname{det}\left(\left.\frac{\psi}{2 \pi}\right|_{\mathcal{E}_{1}^{\alpha}}\right)^{-1} \\
& \times \exp \left[-\mathrm{i}\left(\gamma_{0}, \psi\right)-\frac{\mathrm{i} \epsilon}{2}(\psi, \psi)\right] .
\end{aligned}
$$

Here, $\psi$ is an element in the algebra $\mathfrak{h}_{0}^{\alpha}$. Since the group $H_{0}^{\alpha}=U(1)_{\mathrm{R}} \times$ $G_{\alpha} \times U(1)_{z}$ decomposes as a product, we frequently write $\psi$ in terms of components

$$
\psi=(p, \phi, a) \in \mathbb{R} \oplus \mathfrak{g}_{\alpha} \oplus \mathbb{R},
$$

where $p$ and $a$ generate $U(1)_{\mathrm{R}}$ and $U(1)_{\mathrm{z}}$ respectively, and $\phi$ is an element of $\mathfrak{g}_{\alpha}$.

In arriving at the expression for $\left.Z(\epsilon ; C, R)\right|_{\mathcal{O}_{\alpha} / G}$ in $(7.95)$, we have multiplied the result obtained directly from (6.41) by

$$
\operatorname{Vol}\left[U(1)_{R}\right] \cdot \operatorname{Vol}\left[U(1)_{Z}\right] \cdot 2 \pi \epsilon
$$

which accounts for the prefactor involving $\epsilon$ in (7.95). The same multiplicative factor appears in Section 5 of [18], for precisely the same reason. For sake of brevity, we refer the interested reader to the discussion surrounding (5.10) of [18] for a simple explanation of how this normalization factor arises.

In the remainder of this section, most of our effort will be devoted to evaluating the determinants of $\psi \in \mathfrak{h}_{0}^{\alpha}$ acting on the infinite-dimensional vector spaces $\mathcal{E}_{0}^{\alpha}$ and $\mathcal{E}_{1}^{\alpha}$. However, let us first make the argument of the exponential in (7.95) a bit more explicit.

By definition, $\gamma_{0} \in \mathfrak{h}_{0}^{\alpha}$ is the dual of the value of the moment map $\mu$ evaluated at the point $\alpha \in \mathcal{O}_{\alpha}$. According to (4.105), $\mu$ is generally given on $\overline{\mathcal{A}}_{\alpha}$ by

$$
\begin{aligned}
\langle\mu,(p, \phi, a)\rangle= & a-p \int_{M} \kappa \wedge \operatorname{Tr}\left[\frac{1}{2} £_{\mathrm{R}} A \wedge A+\epsilon \alpha\left(g^{-1} £_{\mathrm{R}} g\right) \delta_{C}\right] \\
& -\int_{M} \kappa \wedge \operatorname{Tr}\left(\phi \mathcal{F}_{A}\right)+\int_{M} d \kappa \wedge \operatorname{Tr}(\phi A) .
\end{aligned}
$$

Points in $\mathcal{O}_{\alpha}$ correspond to classical configurations of $(A, U)$ which are annihilated by $£_{\mathrm{R}}$ and satisfy $\mathcal{F}_{A}=0$, so only the first and last terms in (7.98) contribute when $\mu$ is evaluated at points in $\mathcal{O}_{\alpha}$. 
We compute directly the last term in (7.98) to be

$$
\begin{aligned}
\int_{M} d \kappa \wedge \operatorname{Tr}(\phi \mathrm{A}) & =\int_{M} \kappa \wedge \operatorname{Tr}\left(\phi F_{A}\right), \\
& =-\epsilon \int_{M} \kappa \wedge \delta_{C} \operatorname{Tr}(\alpha \phi), \\
& =-\epsilon \operatorname{Tr}(\alpha \phi)=\epsilon\langle\alpha, \phi\rangle .
\end{aligned}
$$

In deducing the first equality of (7.99), we integrate by parts with $\phi \in \mathfrak{g}_{\alpha}$ constant, and in the second equality, we use that $\mathcal{F}_{A}=F_{A}+\epsilon \alpha \cdot \delta_{C}=0$ at the point $\alpha \in \mathcal{O}_{\alpha}$. Of course, up to the factor of $\epsilon$, we recognize the last expression in (7.99) as nothing more than the moment map (4.18) for the action of $G$ on the coadjoint orbit $\mathcal{O}_{\alpha}$.

From (7.98) and (7.99) we thereby obtain

$$
\left(\gamma_{0}, \psi\right)=\left.\langle\mu,(p, \phi, a)\rangle\right|_{\alpha \in \mathcal{O}_{\alpha}}=a+\epsilon\langle\alpha, \phi\rangle .
$$

We also recall from (3.38) that the norm of $\psi$ is given by

$$
\begin{aligned}
(\psi, \psi) & =-\int_{M} \kappa \wedge d \kappa \operatorname{Tr}\left(\phi^{2}\right)-2 p a, \\
& =-\frac{d}{\mathrm{P}} \operatorname{Tr}\left(\phi^{2}\right)-2 p a .
\end{aligned}
$$

In passing to the second line of (7.101), we use the description of $d \kappa$ in (3.19) along with the identity in (7.30) to compute the integral $\int_{M} \kappa \wedge d \kappa=d / \mathrm{P}$, where $d$ is defined in (7.33) and $\mathrm{P}$ is defined in (7.58). Via (7.100) and (7.101), the integral over $\mathfrak{h}_{0}^{\alpha}$ then takes the more explicit form

$$
\begin{aligned}
\left.Z(\epsilon ; C, R)\right|_{\mathcal{O}_{\alpha} / G} \\
=\frac{(2 \pi \epsilon)}{\operatorname{Vol}\left(G_{\alpha}\right)} \int_{\mathbb{R} \times \mathfrak{g}_{\alpha} \times \mathbb{R}}\left[\frac{d p}{2 \pi}\right]\left[\frac{d \phi}{2 \pi}\right]\left[\frac{d a}{2 \pi}\right] \operatorname{det}\left(\left.\frac{\psi}{2 \pi}\right|_{\mathcal{E}_{0}^{\alpha}}\right) \operatorname{det}\left(\left.\frac{\psi}{2 \pi}\right|_{\mathcal{E}_{1}^{\alpha}}\right)^{-1} \\
\quad \times \exp \left[-\mathrm{i} a-\mathrm{i} \epsilon\langle\alpha, \phi\rangle+\frac{\mathrm{i} \epsilon}{2}\left(\frac{d}{\mathrm{P}}\right) \operatorname{Tr}\left(\phi^{2}\right)+\mathrm{i} \epsilon p a\right] .
\end{aligned}
$$

Let us make one final simplification of (7.102) before we proceed to honest calculations. As we have seen, the vector bundles $\mathcal{E}_{0}^{\alpha}$ and $\mathcal{E}_{1}^{\alpha}$ both decompose into summands associated to the respective factors in the product 
$\overline{\mathcal{A}}_{\alpha}=\overline{\mathcal{A}} \times \epsilon L \mathcal{O}_{\alpha}$, so that

$$
\mathcal{E}_{0}^{\alpha}=\mathcal{E}_{0} \oplus \mathfrak{g}^{(1,0)}, \quad \mathcal{E}_{1}^{\alpha}=\mathcal{E}_{1} \oplus \overline{\mathcal{N}}_{\alpha}
$$

Consequently, in any natural regularization, we can factorize the ratio of determinants appearing in (7.102) as

$$
\operatorname{det}\left(\left.\frac{\psi}{2 \pi}\right|_{\mathcal{E}_{0}^{\alpha}}\right) \cdot \operatorname{det}\left(\left.\frac{\psi}{2 \pi}\right|_{\mathcal{E}_{1}^{\alpha}}\right)^{-1}=e(\overline{\mathcal{A}}) \cdot e\left(L \mathcal{O}_{\alpha}\right)
$$

where we introduce the separate ratios

$$
\begin{aligned}
e(\overline{\mathcal{A}}) & =\operatorname{det}\left(\left.\frac{\psi}{2 \pi}\right|_{\mathcal{E}_{0}}\right) \operatorname{det}\left(\left.\frac{\psi}{2 \pi}\right|_{\mathcal{E}_{1}}\right)^{-1}, \\
e\left(L \mathcal{O}_{\alpha}\right) & =\operatorname{det}\left(\left.\frac{\psi}{2 \pi}\right|_{\mathfrak{g}^{(1,0)}}\right) \operatorname{det}\left(\left.\frac{\psi}{2 \pi}\right|_{\mathcal{N}_{\alpha}}\right)^{-1} .
\end{aligned}
$$

The integral in (7.102) immediately becomes

$$
\begin{aligned}
\left.Z(\epsilon ; C, R)\right|_{\mathcal{O}_{\alpha} / G}= & \frac{(2 \pi \epsilon)}{\operatorname{Vol}\left(G_{\alpha}\right)} \int_{\mathbb{R} \times \mathfrak{g}_{\alpha} \times \mathbb{R}}\left[\frac{d p}{2 \pi}\right]\left[\frac{d \phi}{2 \pi}\right]\left[\frac{d a}{2 \pi}\right] e(\overline{\mathcal{A}}) \cdot e\left(L \mathcal{O}_{\alpha}\right) \\
& \times \exp \left[-\mathrm{i} a-\mathrm{i} \epsilon\langle\alpha, \phi\rangle+\frac{\mathrm{i} \epsilon}{2}\left(\frac{d}{\mathrm{P}}\right) \operatorname{Tr}\left(\phi^{2}\right)+\mathrm{i} \epsilon p a\right] .
\end{aligned}
$$

The essence of localization on $\mathcal{O}_{\alpha} / G$ now lies in evaluating $e(\overline{\mathcal{A}})$ and $e\left(L \mathcal{O}_{\alpha}\right)$.

\subsubsection{Evaluating $e(\overline{\mathcal{A}})$}

Of the determinants in $(7.105), e(\overline{\mathcal{A}})$ is by far the more delicate to compute. Thankfully, we have already evaluated $e(\overline{\mathcal{A}})$ in (5.90) of [18], where we used the standard, but slightly ad hoc, technique of zeta/eta-function regularization to define the infinite products of eigenvalues of $\psi$ acting on $\mathcal{E}_{0}$ and $\mathcal{E}_{1}$. Because the central generator $a$ of $U(1)_{z}$ acts trivially, $e(\overline{\mathcal{A}})$ depends only 
on the generators $(p, \phi)$ of $U(1)_{\mathrm{R}} \times G_{\alpha}$ and is given by ${ }^{33}$

$$
\begin{aligned}
e(\overline{\mathcal{A}})= & \exp \left(-\frac{i \pi}{2} \eta_{0}(0)\right) \cdot \frac{(2 \pi)^{\Delta_{G}}}{(p \sqrt{\mathrm{P}})^{\Delta_{T}}} \exp \left[\frac{\mathrm{i} \check{c}_{\mathfrak{g}}}{4 \pi p^{2}}\left(\frac{d}{\mathrm{P}}\right) \operatorname{Tr}\left(\phi^{2}\right)\right] \\
& \times \prod_{\beta>0}\langle\beta, \phi\rangle^{-2}\left[2 \sin \left(\frac{\langle\beta, \phi\rangle}{2 p}\right)\right]^{2-N} \prod_{j=1}^{N}\left[2 \sin \left(\frac{\langle\beta, \phi\rangle}{2 a_{j} p}\right)\right], \\
\Delta_{G}= & \operatorname{dim} G, \quad \Delta_{T}=\operatorname{dim} T .
\end{aligned}
$$

Here, we recall that $T \subset G$ is a maximal torus, and in writing this formula for $e(\overline{\mathcal{A}})$, we assume without loss that $\phi$ lies in the associated Cartan subalgebra t. Each $\beta>0$ is then a positive root of $G$, and $\langle\cdot, \cdot\rangle$ is the canonical dual pairing.

One very interesting feature of $e(\overline{\mathcal{A}})$ is the appearance of a phase proportional to $\operatorname{Tr}\left(\phi^{2}\right)$ in the second line of (7.107). This phase also involves the dual Coxeter number $\check{c}_{\mathfrak{g}}$ of the Lie algebra $\mathfrak{g}$, which for convenience we take to be simply-laced. ${ }^{34}$ As we discussed in detail in [18], the $\phi$-dependent phase in $e(\overline{\mathcal{A}})$ ultimately leads to the famous quantum shift $k \rightarrow k+\check{c}_{\mathfrak{g}}$ in the Chern-Simons level. We will shortly encounter a closely related quantum effect when we compute $e\left(L \mathcal{O}_{\alpha}\right)$.

Finally, $\eta_{0}(0)$ in $(7.107)$ is a rather subtle constant that arises when we introduce an eta-function to define the phase of $e(\overline{\mathcal{A}})$. In the simplest case that $M$ is a circle bundle of degree $n$ over a smooth Riemann surface $\Sigma$, we computed $\eta_{0}(0)$ in (5.83) of [18] to be

$$
\eta_{0}(0)=-\frac{n \Delta_{G}}{6}, \quad \Sigma \text { smooth }
$$

independent of the genus of $\Sigma$.

On the other hand, to describe a non-trivial torus knot $\mathcal{K}_{\mathbf{p}, \mathbf{q}} \subset S^{3}$, we consider a Seifert structure on $S^{3}$ for which the base $\Sigma=\mathbb{W} \mathbb{C P}_{\mathbf{p}, \mathbf{q}}^{1}$ is a nontrivial orbifold. As we ultimately wish to compare the empirical formula for $Z\left(\epsilon ; \mathcal{K}_{\mathbf{p}, \mathbf{q}}, \mathbf{j}\right)$ in $(7.73)$ with precise results of localization, we need to determine the value of $\eta_{0}(0)$ in the orbifold case as well.

Here, we are in luck. By construction, $\eta_{0}(0)$ is the eta-invariant associated to a certain "adiabatic" Dirac operator considered by Nicolaescu [89] in the

\footnotetext{
${ }^{33}$ Strictly speaking, we computed $e(\overline{\mathcal{A}})$ in [18] for the case that $\phi$ lies in the Lie algebra of $G$, as opposed to the subgroup $G_{\alpha} \subset G$, but the result in [18] immediately specializes.

${ }^{34}$ See $(5.88)$ of [18] for a description of the $\phi$-dependent phase which does not require $\mathfrak{g}$ to be simply-laced.
} 
"ATMP-17-1-A1-BEA" — 2013/5/15 — 19:32 — page 153 — \#153

context of Seiberg-Witten theory on a four-manifold bounding the Seifert manifold $M$. According to Proposition 1.4 of [89], the value of $\eta_{0}(0)$ for a general Seifert manifold $M$ is given by

$$
\eta_{0}(0)=\frac{\Delta_{G}}{6}\left[-c_{1}(\mathcal{L})+12 \sum_{j=1}^{N} s\left(b_{j}, a_{j}\right)\right] .
$$

In (7.109), $\mathcal{L}$ is the line $V$-bundle associated to the Seifert presentation of $M$. Explicitly in terms of Seifert invariants,

$$
c_{1}(\mathcal{L})=n+\sum_{j=1}^{N} \frac{b_{j}}{a_{j}}=\frac{d}{\mathrm{P}},
$$

where the final equality in (7.110) holds when $M$ is a Seifert homology sphere or a cyclic $\mathbb{Z}_{d}$ quotient thereof.

Of particular note, the general formula (7.109) for $\eta_{0}(0)$ now involves the Dedekind sum $s\left(b_{j}, a_{j}\right)$. The appearance of such a complicated arithmetic object in a one-loop determinant may seem rather mysterious, but it is crucial if we are to make contact with empirical formulae such as (7.64), in which the Dedekind sum enters through the phase $\theta_{0}$. That said, at the moment we do not wish to divert the exposition to review the complete derivation of (7.109). Instead, we refer the interested reader to Appendix C for a self-contained computation of the adiabatic eta-invariant $\eta_{0}(0)$.

\subsubsection{Evaluating $e\left(L \mathcal{O}_{\alpha}\right)$}

We are left to evaluate the product of determinants associated to the free loopspace $L \mathcal{O}_{\alpha}$,

$$
e\left(L \mathcal{O}_{\alpha}\right)=\operatorname{det}\left(\left.\frac{\psi}{2 \pi}\right|_{\mathfrak{g}^{(1,0)}}\right) \operatorname{det}\left(\left.\frac{\psi}{2 \pi}\right|_{\overline{\mathcal{N}}_{\alpha}}\right)^{-1} .
$$

Eventually these determinants, along with the moment map on $\mathcal{O}_{\alpha}$ which enters the argument of the exponential in (7.106), will determine the invariant function of $\phi$ which represents the Seifert loop operator under localization at the trivial connection on $M$. 
According to (4.86), the vector field generated by an arbitrary element $\psi \equiv(p, \phi, a)$ in the Lie algebra of the Hamiltonian group $\mathcal{H}$ is given on $L \mathcal{O}_{\alpha}$ by

$$
\delta g=p £_{\mathrm{R}} g+\left.\phi\right|_{C} \cdot g .
$$

Upon restriction to the stabilizer $\mathfrak{h}_{0}^{\alpha}=\mathbb{R} \oplus \mathfrak{g}_{\alpha} \oplus \mathbb{R}$, the generator $\psi$ consequently acts on both the finite-dimensional vector space $\mathfrak{g}^{(1,0)}$ and the infinite-dimensional vector space $\overline{\mathcal{N}}_{\alpha}$ as the first-order differential operator

$$
\mathcal{D}_{(p, \phi)}=p £_{\mathrm{R}}+[\phi, \cdot] .
$$

In complete analogy to the evaluation of $e(\overline{\mathcal{A}})$ in Section 5.2 of [18] (see also Appendix C), we now compute the respective determinants of $\mathcal{D}_{(p, \phi)}$ acting on $\mathfrak{g}^{(1,0)}$ and $\overline{\mathcal{N}}_{\alpha}$.

We begin by evaluating the numerator of (7.111), which is merely a finitedimensional determinant. Elements in $\mathfrak{g}^{(1,0)}$ represent holomorphic tangent vectors to $\mathcal{O}_{\alpha}$ embedded as the space of constant maps in $L \mathcal{O}_{\alpha}$. Hence the Lie derivative $£_{\mathrm{R}}$ annihilates $\mathfrak{g}^{(1,0)}$, so trivially

$$
\begin{aligned}
\operatorname{det}\left(\left.\frac{\psi}{2 \pi}\right|_{\mathfrak{g}^{(1,0)}}\right) & =\operatorname{det}\left(\left.\frac{\mathcal{D}_{(p, \phi)}}{2 \pi}\right|_{\mathfrak{g}^{(1,0)}}\right), \\
& =\operatorname{det}\left(\left.\frac{[\phi, \cdot]}{2 \pi}\right|_{\mathfrak{g}^{(1,0)}}\right), \quad \phi \in \mathfrak{g}_{\alpha} .
\end{aligned}
$$

To compute the latter determinant in (7.114), we assume without loss that $\phi$ lies in the Cartan subalgebra $\mathfrak{t} \subseteq \mathfrak{g}_{\alpha}$ associated to a maximal torus $T \subseteq G_{\alpha} \cdot{ }^{35}$ With this assumption, the adjoint action of $\phi$ is diagonalized in terms of the roots $\beta$ of $\mathfrak{g}$ as $\left[\phi, x_{\beta}\right]=i\langle\beta, \phi\rangle x_{\beta}$, where $x_{\beta}$ is an element of the rootspace $\mathfrak{e}_{\beta}$. Thus,

$$
\begin{aligned}
\operatorname{det}\left(\left.\frac{[\phi, \cdot]}{2 \pi}\right|_{\mathfrak{g}^{(1,0)}}\right) & =\prod_{\left(\beta_{+}, \alpha\right)>0}\left(\frac{\mathrm{i}}{2 \pi}\left\langle\beta_{+}, \phi\right\rangle\right) \\
& =\left(\frac{\mathrm{i}}{2 \pi}\right)^{\left(\Delta_{G}-\Delta_{G_{\alpha}}\right) / 2} \cdot \prod_{\left(\beta_{+}, \alpha\right)>0}\left\langle\beta_{+}, \phi\right\rangle, \\
\Delta_{G} & =\operatorname{dim} G, \quad \Delta_{G_{\alpha}}=\operatorname{dim} G_{\alpha} .
\end{aligned}
$$

\footnotetext{
${ }^{35}$ If the weight $\alpha$ happens to be regular, then $\mathfrak{g}_{\alpha}=\mathfrak{t}$ is automatically such a Cartan subalgebra.
} 
As indicated, the products in (7.115) run over those roots $\beta_{+}$whose associated rootspaces lie in the holomorphic tangent space $\mathfrak{g}^{(1,0)}$ to $\mathcal{O}_{\alpha}$. Equivalently, each root $\beta_{+}$satisfies $\left(\beta_{+}, \alpha\right)>0$. Of course, if $\alpha$ is regular, the latter inequality just says that $\beta_{+}>0$ is a positive root. At the opposite extreme, for $\alpha=0$ and $\mathfrak{g}^{(1,0)}$ empty, the product over $\beta_{+}$is trivial.

Because the dimension of the normal bundle to $\mathcal{O}_{\alpha}$ inside $L \mathcal{O}_{\alpha}$ is infinite, we must work somewhat harder to evaluate the denominator in (7.111). We recall that $\overline{\mathcal{N}}_{\alpha}$ decomposes as a direct sum of anti-holomorphic tangent spaces $\mathfrak{g}_{t}^{(0,1)}$ upon which $£_{\mathrm{R}}$ acts with eigenvalue $-2 \pi i t$,

$$
\overline{\mathcal{N}}_{\alpha}=\bigoplus_{t \geq 1}\left[\mathfrak{g}_{t}^{(0,1)} \oplus \mathfrak{g}_{-t}^{(0,1)}\right]
$$

Diagonalizing the action of $\phi$ on each summand $\mathfrak{g}_{t}^{(0,1)}$, we write the determinant of $\mathcal{D}_{(p, \phi)}$ acting on $\overline{\mathcal{N}}_{\alpha}$ formally as the product

$$
\begin{aligned}
\operatorname{det}\left(\left.\frac{\psi}{2 \pi}\right|_{\overline{\mathcal{N}}_{\alpha}}\right)= & \operatorname{det}\left(\left.\frac{\mathcal{D}_{(p, \phi)}}{2 \pi}\right|_{\overline{\mathcal{N}}_{\alpha}}\right) \\
= & \prod_{t \neq 0} \prod_{\left(\beta_{+}, \alpha\right)>0}\left[\left(-i t p-\frac{\mathrm{i}}{2 \pi}\left\langle\beta_{+}, \phi\right\rangle\right)\right] \\
= & \exp \left(-\frac{\mathrm{i} \pi}{2} \delta(p, \phi)\right) \times \prod_{t \geq 1} \mid(t p)^{\left(\Delta_{G}-\Delta_{G_{\alpha}}\right)} \\
& \times \prod_{\left(\beta_{+}, \alpha\right)>0}\left(1-\left(\frac{\left\langle\beta_{+}, \phi\right\rangle}{2 \pi t p}\right)^{2}\right) \mid
\end{aligned}
$$

In the second line of (7.117), we express the determinant of $\phi$ acting on $\mathfrak{g}_{t}^{(0,1)}$ as a product over positive roots $\beta_{+}>0$, with a crucial sign relative to the analogous determinant in (7.115) to account for the exchange $\mathfrak{g}_{t}^{(1,0)} \leftrightarrow \mathfrak{g}_{t}^{(0,1)}$. In the final line of (7.117), we then encode the phase of the determinant through a function $\delta(p, \phi)$ generally depending upon both $p$ and $\phi$. Otherwise, the terms which appear explicitly in the product over the Fourier mode $t$ represent the norm.

As often the case for functional determinants, the norm in (7.117) is much easier to evaluate than the phase $\delta(p, \phi)$, so we will compute the norm first. 
"ATMP-17-1-A1-BEA" — 2013/5/15 — 19:32 — page 156 — \#156

To evaluate the product over $t$, we recall the well-known identity

$$
\frac{\sin (x)}{x}=\prod_{t \geq 1}\left(1-\frac{x^{2}}{\pi^{2} t^{2}}\right)
$$

implying

$$
\prod_{t \geq 1}\left(1-\left(\frac{\left\langle\beta_{+}, \phi\right\rangle}{2 \pi t p}\right)^{2}\right)=\frac{2 p}{\left\langle\beta_{+}, \phi\right\rangle} \sin \left(\frac{\left\langle\beta_{+}, \phi\right\rangle}{2 p}\right) .
$$

Just as for the computation in (5.65) of [18], we then use the Riemann zeta-function $\zeta(s)$ to define the trivial but infinite products

$$
\begin{aligned}
& \prod_{t \geq 1} p^{\left(\Delta_{G}-\Delta_{G_{\alpha}}\right)}=\exp \left[\left(\Delta_{G}-\Delta_{G_{\alpha}}\right) \ln p \cdot \zeta(0)\right]=p^{-\left(\Delta_{G}-\Delta_{G_{\alpha}}\right) / 2} \\
& \prod_{t \geq 1} t^{\left(\Delta_{G}-\Delta_{G_{\alpha}}\right)}=\exp \left[-\left(\Delta_{G}-\Delta_{G_{\alpha}}\right) \cdot \zeta^{\prime}(0)\right]=(2 \pi)^{\left(\Delta_{G}-\Delta_{G_{\alpha}}\right) / 2} .
\end{aligned}
$$

Canceling overall factors of $p$ from (7.119) and (7.120), we obtain

$$
\begin{aligned}
\operatorname{det}\left(\left.\frac{\psi}{2 \pi}\right|_{\mathcal{N}_{\alpha}}\right)= & (2 \pi)^{\left(\Delta_{G}-\Delta_{G_{\alpha}}\right) / 2} \exp \left(-\frac{\mathrm{i} \pi}{2} \delta(p, \phi)\right) \\
& \times \prod_{\left(\beta_{+}, \alpha\right)>0}\left|\frac{2}{\left\langle\beta_{+}, \phi\right\rangle} \sin \left(\frac{\left\langle\beta_{+}, \phi\right\rangle}{2 p}\right)\right| .
\end{aligned}
$$

Our remaining task is to compute the phase $\delta(p, \phi)$ in (7.117). Naively, $\delta(p, \phi)$ is given by the sum

$$
\delta(p, \phi) \approx \sum_{t \neq 0} \sum_{\left(\beta_{+}, \alpha\right)>0} \operatorname{sign}\left(\lambda\left(t, \beta_{+}\right)\right), \quad \lambda\left(t, \beta_{+}\right)=t+\frac{\left\langle\beta_{+}, \phi\right\rangle}{2 \pi p} .
$$

We have not written the expression in (7.122) with an equality because the sum over eigenvalues $\lambda(t, \beta)$ is ill-defined without a regulator. To make sense of (7.122), we follow the philosophy of [14] and introduce an eta-function associated to the spectrum of $\mathcal{D}_{(p, \phi)}$. We thus set

$$
\delta_{(p, \phi)}(s)=\sum_{t \neq 0} \sum_{\left(\beta_{+}, \alpha\right)>0} \operatorname{sign}\left(\lambda\left(t, \beta_{+}\right)\right)\left|\lambda\left(t, \beta_{+}\right)\right|^{-s} .
$$

Here $s$ is a complex parameter. When the real part of $s$ is sufficiently large, the sum in (7.123) is absolutely convergent, so that $\delta_{(p, \phi)}(s)$ is defined in 
that case. Otherwise, $\delta_{(p, \phi)}(s)$ is defined by analytic continuation in the $s$-plane. Assuming that $\delta_{(p, \phi)}(s)$ remains finite as $s \rightarrow 0$, we then take

$$
\delta(p, \phi)=\delta_{(p, \phi)}(0)
$$

To evaluate $\delta_{(p, \phi)}(s)$ near $s=0$, we first expand the sum in (7.123) as

$$
\delta_{(p, \phi)}(s)=\sum_{t \geq 1} \sum_{\left(\beta_{+}, \alpha\right)>0} \frac{1}{\left(t+\frac{\left\langle\beta_{+}, \phi\right\rangle}{2 \pi p}\right)^{s}}-\sum_{t \geq 1} \sum_{\left(\beta_{+}, \alpha\right)>0} \frac{1}{\left(t-\frac{\left\langle\beta_{+}, \phi\right\rangle}{2 \pi p}\right)^{s}} .
$$

Here we assume that $p$ and $\phi$ satisfy

$$
0<\frac{\left\langle\beta_{+}, \phi\right\rangle}{2 \pi p}<1
$$

for each root $\beta_{+}$appearing in the sum. Otherwise, when the quantity in (7.126) shifts by an integer, the phase $\exp (-\mathrm{i} \pi \delta(p, \phi) / 2)$ is multiplied by a sign \pm 1 , depending upon the parity of the shift. This sign effectively removes the absolute value bars $|\cdot|$ appearing in (7.121), so that the determinant of $\mathcal{D}_{(p, \phi)}$ depends analytically on $p$ and $\phi$ as one might naively expect.

We now apply the binomial expansion to the denominators in (7.125) to obtain

$$
\delta_{(p, \phi)}(s)=\sum_{t \geq 1} \sum_{\left(\beta_{+}, \alpha\right)>0}-\frac{2 s}{t^{s+1}} \cdot\left(\frac{\left\langle\beta_{+}, \phi\right\rangle}{2 \pi p}\right)+\sum_{t \geq 1} \sum_{\left(\beta_{+}, \alpha\right)>0} s \cdot \mathcal{O}\left(\frac{1}{t^{s+2}}\right) .
$$

In the process of expanding (7.125), we collect into $\mathcal{O}\left(1 / t^{s+2}\right)$ all terms for which the sum over $t$ is absolutely convergent near $s=0$. As a result, when we evaluate $\delta_{(p, \phi)}(s)$ at $s=0$, the last term in (7.127) vanishes.

On the other hand, we note that

$$
\sum_{t \geq 1} \sum_{\left(\beta_{+}, \alpha\right)>0}-\frac{2 s}{t^{s+1}} \cdot\left(\frac{\left\langle\beta_{+}, \phi\right\rangle}{2 \pi p}\right)=-s \zeta(1+s) \cdot \sum_{\left(\beta_{+}, \alpha\right)>0} \frac{\left\langle\beta_{+}, \phi\right\rangle}{\pi p},
$$

where $\zeta$ is again the Riemann zeta-function. Because $\zeta(1+s)$ has a simple pole with unit residue at $s=0$, we see that (7.128) makes a non-zero 
contribution to $\delta_{(p, \phi)}(0)$. Specifically,

$$
\delta_{(p, \phi)}(0)=-\frac{2\left\langle\rho^{[\alpha]}, \phi\right\rangle}{\pi p} \bmod 2,
$$

where we introduce the generalized Weyl vector

$$
\rho^{[\alpha]}=\frac{1}{2} \sum_{\left(\beta_{+}, \alpha\right)>0} \beta_{+} .
$$

If $\alpha$ is regular, the sum which defines $\rho^{[\alpha]}$ runs over all positive roots of $\mathfrak{g}$, and $\rho^{[\alpha]}=\rho$ is the distinguished weight which appeared previously in (7.27). This observation motivates the otherwise extraneous factor of $1 / 2$ in the definition of $\rho^{[\alpha]}$. Otherwise, if $\alpha$ is not regular, the sum which defines $\rho^{[\alpha]}$ runs over only the subset of positive roots whose corresponding rootspaces lie in $\mathfrak{g}^{(1,0)}$. In this situation, $\rho^{[\alpha]}$ need not be a weight of $G$, as for instance when $G=S U(3)$ and $\alpha$ is the weight of the fundamental representation 3. Trivially, in the extreme case $\alpha=0$, then $\rho^{[\alpha]}=0$ as well.

Finally, we emphasize that our computation of $\delta_{(p, \phi)}(0)$ is strictly valid modulo 2, due to the assumption in (7.126). As we mentioned before, a complete formula for $\delta_{(p, \phi)}(0)$ also includes locally-constant terms which vanish mod 2 and which compensate for the absolute value bars $|\cdot|$ appearing in (7.121).

Physically, the non-trivial value for $\delta_{(p, \phi)}(0)$ in (7.129) can be understood as a finite renormalization effect, due to the divergence in the naive sum over eigenvalues in (7.122). In complete analogy to the $\phi$-dependent phase appearing in (7.107), the determinant of $\psi \equiv \mathcal{D}_{(p, \phi)}$ acting on $\mathcal{N}_{\alpha}$ acquires a similar $\phi$-dependent quantum phase,

$$
\begin{aligned}
\operatorname{det}\left(\left.\frac{\psi}{2 \pi}\right|_{\mathcal{N}_{\alpha}}\right)= & (2 \pi)^{\left(\Delta_{G}-\Delta_{G_{\alpha}}\right) / 2} \exp \left(\mathrm{i} \frac{\left\langle\rho^{[\alpha]}, \phi\right\rangle}{p}\right) \\
& \times \prod_{\left(\beta_{+}, \alpha\right)>0} \frac{2}{\left\langle\beta_{+}, \phi\right\rangle} \sin \left(\frac{\left\langle\beta_{+}, \phi\right\rangle}{2 p}\right) .
\end{aligned}
$$

Taking the ratio between the determinants in (7.115) and (7.131), we see that $e\left(L \mathcal{O}_{\alpha}\right)$ is given succinctly by

$$
e\left(L \mathcal{O}_{\alpha}\right)=\left(\frac{1}{2 \pi}\right)^{\left(\Delta_{G}-\Delta_{G_{\alpha}}\right)} \exp \left[\frac{\mathrm{i} \pi}{4}\left(\Delta_{G}-\Delta_{G_{\alpha}}\right)-\mathrm{i} \frac{\left\langle\rho^{[\alpha]}, \phi\right\rangle}{p}\right]
$$




$$
\begin{aligned}
& \times \prod_{\left(\beta_{+}, \alpha\right)>0}\left\langle\beta_{+}, \phi\right\rangle^{2} \cdot\left[2 \sin \left(\frac{\left\langle\beta_{+}, \phi\right\rangle}{2 p}\right)\right]^{-1}, \\
\Delta_{G}= & \operatorname{dim} G, \quad \Delta_{G_{\alpha}}=\operatorname{dim} G_{\alpha} .
\end{aligned}
$$

\subsubsection{Simplifying the integral over $\mathfrak{h}_{0}^{\alpha}$}

As manifest in (7.107) and (7.132), neither $e(\overline{\mathcal{A}})$ nor $e\left(L \mathcal{O}_{\alpha}\right)$ depends upon the variable $a$, which parameterizes the Lie algebra of $U(1)_{z}$. Because $U(1)_{z}$ acts in a completely trivial fashion on $\overline{\mathcal{A}}_{\alpha}$, the result could hardly have been otherwise. Yet this observation does have an important consequence.

We recall from (7.106) that the local contribution from $\left\{\varrho_{\mathrm{ab}}\right\} \cong \mathcal{O}_{\alpha} / G$ to the Seifert loop path integral is given by

$$
\begin{aligned}
\left.Z(\epsilon ; C, R)\right|_{\mathcal{O}_{\alpha} / G}= & \frac{(2 \pi \epsilon)}{\operatorname{Vol}\left(G_{\alpha}\right)} \int_{\mathbb{R} \times \mathfrak{g}_{\alpha} \times \mathbb{R}}\left[\frac{d p}{2 \pi}\right]\left[\frac{d \phi}{2 \pi}\right]\left[\frac{d a}{2 \pi}\right] e(\overline{\mathcal{A}}) \cdot e\left(L \mathcal{O}_{\alpha}\right) \\
& \times \exp \left[-\mathrm{i} a-\mathrm{i} \epsilon\langle\alpha, \phi\rangle+\frac{\mathrm{i} \epsilon}{2}\left(\frac{d}{\mathrm{P}}\right) \operatorname{Tr}\left(\phi^{2}\right)+\mathrm{i} \epsilon p a\right] .
\end{aligned}
$$

Since $a$ enters the integrand of (7.133) only linearly in the argument of the exponential, we can immediately integrate over $a$ using the elementary identity

$$
\int_{-\infty}^{+\infty} d y \exp (-\mathrm{i} x y)=2 \pi \delta(x) .
$$

Hence, the integral over $a$ yields a delta-function $2 \pi \delta(1-\epsilon p)$.

Next, we use the delta-function to perform the integral over $p$, thereby setting $p=1 / \epsilon$. In the process, the prefactor of $2 \pi \epsilon$ which appears in the normalization of (7.133) is canceled, and the integral over $\mathbb{R} \oplus \mathfrak{g}_{\alpha} \oplus \mathbb{R}$ reduces to an integral over $\mathfrak{g}_{\alpha}$ alone,

$$
\begin{aligned}
& \left.Z(\epsilon ; C, R)\right|_{\mathcal{O}_{\alpha} / G} \\
& =\exp \left[-\frac{\mathrm{i} \pi}{2}\left(\eta_{0}(0)-\frac{1}{2}\left(\Delta_{G}-\Delta_{G_{\alpha}}\right)\right)\right] \frac{1}{\operatorname{Vol}\left(G_{\alpha}\right)}\left(\frac{\epsilon}{\sqrt{\mathrm{P}}}\right)^{\Delta_{T}} \\
& \quad \times \int_{\mathfrak{g}_{\alpha}}[d \phi] \exp \left[-\mathrm{i} \epsilon\left\langle\alpha+\rho^{[\alpha]}, \phi\right\rangle+\frac{\mathrm{i} \epsilon}{2}\left(\frac{d}{\mathrm{P}}\right)\left(1+\frac{\epsilon \check{c}_{\mathfrak{g}}}{2 \pi}\right) \operatorname{Tr}\left(\phi^{2}\right)\right]
\end{aligned}
$$




$$
\begin{aligned}
& \times \prod_{\beta>0}\langle\beta, \phi\rangle^{-2}\left[2 \sin \left(\frac{\epsilon\langle\beta, \phi\rangle}{2}\right)\right]^{2-N} \prod_{j=1}^{N}\left[2 \sin \left(\frac{\epsilon\langle\beta, \phi\rangle}{2 a_{j}}\right)\right] \\
& \times \prod_{\left(\beta_{+}, \alpha\right)>0}\left\langle\beta_{+}, \phi\right\rangle^{2}\left[2 \sin \left(\frac{\epsilon\left\langle\beta_{+}, \phi\right\rangle}{2}\right)\right]^{-1} .
\end{aligned}
$$

Here we have substituted the expressions for $e(\overline{\mathcal{A}})$ and $e\left(L \mathcal{O}_{\alpha}\right)$ in $(7.107)$ and (7.132). Also, as a word of caution, we emphasize that the products over $\beta$ and $\beta_{+}$in (7.135) run over distinct sets of roots whenever $\alpha$ is not regular.

Let us simplify (7.135) a bit further.

If the weight $\alpha$ is regular, then $\mathfrak{g}_{\alpha} \cong \mathfrak{t}$, and the integral in (7.135) automatically runs over the Cartan subalgebra of $G$. More generally, to reduce (7.135) to an integral over $\mathfrak{t}$ even when $\alpha$ is not regular, we note that the integrand is a function on $\mathfrak{g}_{\alpha}$ which is invariant under the adjoint action of $G_{\alpha}$. This invariance ultimately follows from the trivial invariance of $\alpha$ under the Weyl group $\mathfrak{W}_{\alpha}$ of $G_{\alpha}$. Here we think of $\mathfrak{W}_{\alpha}$ as a subgroup of the Weyl group $\mathfrak{W}$ of $G$, and we note that $\mathfrak{W}_{\alpha}$ preserves the set of roots $\beta_{+}$satisfying $\left(\beta_{+}, \alpha\right)>0$. Hence both $\rho^{[\alpha]}$ and the product over roots $\beta_{+}$in the last line of (7.135) are invariant under $\mathfrak{W}_{\alpha}$. The remaining terms in the integrand of (7.135) are manifestly invariant under $\mathfrak{W}_{\alpha}$, from which we deduce invariance under the group $G_{\alpha}$.

Since the integrand of (7.135) is invariant under the adjoint action of $G_{\alpha}$, we can apply the Weyl integral formula to reduce the integral from $\mathfrak{g}_{\alpha}$ to $\mathfrak{t}$. In its infinitesimal version, the Weyl integral formula generally states that if $f$ is a function on a Lie algebra $\mathfrak{g}$ invariant under the adjoint action of a group $G$, then

$$
\int_{\mathfrak{g}}[d \phi] f(\phi)=\frac{1}{|\mathfrak{W}|} \frac{\operatorname{Vol}(G)}{\operatorname{Vol}(T)} \int_{\mathfrak{t}}[d \phi] \prod_{\beta>0}\langle\beta, \phi\rangle^{2} f(\phi) .
$$

Here $|\mathfrak{W}|$ is the order of the Weyl group of $G$, and the product over positive roots $\beta$ of $G$ appearing on the right in (7.136) is a Jacobian factor generalizing the classical van der Monde determinant.

In the case at hand, we want to apply the Weyl integral formula (7.136) not for $G$ but for $G_{\alpha}$. The roots of $G_{\alpha}$ are precisely those roots $\beta_{\perp}$ of $G$ orthogonal to $\alpha$ in the invariant metric on $\mathfrak{t}^{*}$, such that

$$
\left(\beta_{\perp}, \alpha\right)=0 .
$$


Consequently, when we apply the Weyl integral formula to reduce the integral in (7.135) from $\mathfrak{g}_{\alpha}$ to $\mathfrak{t}$, the Weyl Jacobian for $G_{\alpha}$ conspires to cancel against the following product of factors in (7.135):

$$
\prod_{\beta>0}\langle\beta, \phi\rangle^{-2} \cdot \prod_{\left(\beta_{+}, \alpha\right)>0}\left\langle\beta_{+}, \phi\right\rangle^{2}=\prod_{\beta_{\perp}>0}\left\langle\beta_{\perp}, \phi\right\rangle^{-2}
$$

implying

$$
\begin{aligned}
\left.Z(\epsilon ; C, R)\right|_{\mathcal{O}_{\alpha} / G} \\
=\exp \left[-\frac{\mathrm{i} \pi}{2}\left(\eta_{0}(0)-\frac{1}{2}\left(\Delta_{G}-\Delta_{G_{\alpha}}\right)\right)\right] \frac{1}{\left|\mathfrak{W}_{\alpha}\right|} \frac{1}{\operatorname{Vol}(T)}\left(\frac{1}{\sqrt{\mathrm{P}}}\right)^{\Delta_{T}} \\
\quad \times \int_{\mathfrak{t}}[d \phi] \exp \left[-\mathrm{i}\left\langle\alpha+\rho^{[\alpha]}, \phi\right\rangle+\frac{\mathrm{i}}{2 \epsilon_{\mathrm{r}}}\left(\frac{d}{\mathrm{P}}\right) \operatorname{Tr}\left(\phi^{2}\right)\right] \\
\quad \times \prod_{\beta>0}\left[2 \sin \left(\frac{\langle\beta, \phi\rangle}{2}\right)\right]^{2-N} \prod_{j=1}^{N}\left[2 \sin \left(\frac{\langle\beta, \phi\rangle}{2 a_{j}}\right)\right] \\
\quad \times \prod_{\left(\beta_{+}, \alpha\right)>0}\left[2 \sin \left(\frac{\left\langle\beta_{+}, \phi\right\rangle}{2}\right)\right]^{-1}
\end{aligned}
$$

In passing to (7.139), we have performed a change of variables $\phi \mapsto \epsilon \phi$ to remove extraneous factors of $\epsilon$. In the process, we introduce the renormalized coupling $\epsilon_{\mathrm{r}}$,

$$
\epsilon_{\mathrm{r}}=\frac{2 \pi}{k+\check{c}_{\mathfrak{g}}}
$$

to absorb the explicit shift in the coefficient of $\operatorname{Tr}\left(\phi^{2}\right)$ that arises from $e(\overline{\mathcal{A}})$ in (7.107). Also, as hopefully clear, $\left|\mathfrak{W}_{\alpha}\right|$ denotes the order of the Weyl group of $G_{\alpha}$. If $G_{\alpha}=T$ is abelian, then $\mathfrak{W}_{\alpha}$ is trivial and $\left|\mathfrak{W}_{\alpha}\right|=1$.

As a small check, if $\alpha=0$, so that no Wilson loop operator is actually present in the path integral, the expression for $\left.Z(\epsilon ; C, R)\right|_{\mathcal{O}_{\alpha} / G}$ in (7.139) directly reduces to the expression in (5.97) of [18] for the local contribution $\left.Z(\epsilon)\right|_{\{0\}}$ from the trivial connection to the Chern-Simons partition function.

Following [18], we now make two further substitutions to relate the formula in (7.139) to the empirical result in (7.64). First, we rotate the contour 
of integration from $\mathfrak{t} \equiv \mathfrak{t} \times \mathbb{R}$ to $\mathfrak{t} \times \mathrm{e}^{-\frac{\mathrm{i} \pi}{4}}$. Second, we make a change of variables $\phi \mapsto \mathrm{i} \phi$. Hence,

$$
\begin{aligned}
\left.Z(\epsilon ; C, R)\right|_{\mathcal{O}_{\alpha} / G} \\
=\exp \left(-\frac{\mathrm{i} \pi}{2} \eta_{0}(0)\right) \frac{1}{\left|\mathfrak{W}_{\alpha}\right|} \frac{(-1)^{\left(\Delta_{G_{\alpha}}-\Delta_{T}\right) / 2}}{\operatorname{Vol}(T)}\left(\frac{1}{\mathrm{i} \sqrt{\mathrm{P}}}\right)^{\Delta_{T}} \\
\quad \times \int_{\mathfrak{t} \times \mathcal{C}^{(0)}}[d \phi] \exp \left[-\left\langle\alpha+\rho^{[\alpha]}, \phi\right\rangle-\frac{\mathrm{i}}{2 \epsilon_{\mathrm{r}}}\left(\frac{d}{\mathrm{P}}\right) \operatorname{Tr}\left(\phi^{2}\right)\right] \\
\quad \times \prod_{\beta>0}\left[2 \sinh \left(\frac{\langle\beta, \phi\rangle}{2}\right)\right]^{2-N} \prod_{j=1}^{N}\left[2 \sinh \left(\frac{\langle\beta, \phi\rangle}{2 a_{j}}\right)\right] \\
\quad \times \prod_{\left(\beta_{+}, \alpha\right)>0}\left[2 \sinh \left(\frac{\left\langle\beta_{+}, \phi\right\rangle}{2}\right)\right]^{-1} .
\end{aligned}
$$

This contour integral should be compared to (5.99) in [18], to which (7.141) reduces when $\alpha=\rho^{[\alpha]}=0$.

\subsubsection{The Seifert loop operator as a character}

We now arrive at the second main result of this paper - the interpretation of the Seifert loop operator as a character of $G$, via the Weyl character formula. The appearance of the character formula in the context of localization on the loopspace $L \mathcal{O}_{\alpha}$ is not so surprising, given the classic Atiyah-Bott derivation [15] of the character formula from index theory on $\mathcal{O}_{\alpha}$ and the general relation between index theory on a manifold and localization on its loopspace $[9,38]$. Nonetheless, the emergence of the character formula from the Seifert loop path integral is quite satisfying. See also $[5,99]$ for earlier and somewhat more direct quantum mechanical derivations of the character formula.

To start, let us quickly recall the essentials of the character formula. See for instance Section 24 of [52] for a more thorough introduction to the Weyl character formula and a few of its mathematical applications.

We first introduce some notation. For each weight $\alpha$ of $G$, we define an alternating function $\mathrm{A}_{\alpha}$ on the Cartan subalgebra $\mathfrak{t} \subset \mathfrak{g}$ by

$$
\mathrm{A}_{\alpha}(\phi)=\sum_{w \in \mathfrak{W}}(-1)^{w} \mathrm{e}^{\langle w \cdot \alpha, \phi\rangle}, \quad \phi \in \mathfrak{t} .
$$


Here the sum runs over all elements $w$ in the Weyl group $\mathfrak{W}$ of $G$, with a sign $(-1)^{w}$ defined as follows. Because $\mathfrak{t}$ carries a metric invariant under $\mathfrak{W}$, each element $w \in \mathfrak{W}$ acts as an orthogonal transformation with respect to any orthonormal basis of $\mathfrak{t}$. Given such a basis, we then set $(-1)^{w} \equiv \operatorname{det} w= \pm 1$, depending upon whether $w$ preserves or reverses the orientation of $\mathfrak{t}$. As usual, $w \cdot \alpha$ indicates the image of $\alpha$ under the dual action of $\mathfrak{W}$ on $\mathfrak{t}^{*}$. Last but not least, because of the sign $(-1)^{w}$ appearing in (7.142), $\mathrm{A}_{\alpha}$ is alternating under the Weyl action on $\mathfrak{t}$,

$$
\mathrm{A}_{\alpha}(w \cdot \phi)=(-1)^{w} \mathrm{~A}_{\alpha}(\phi), \quad w \in \mathfrak{W} .
$$

In terms of $\mathrm{A}_{\alpha}$, the character $\mathrm{ch}_{R}$ associated to each irreducible representation $R$ of $G$ has a remarkably simple description. As a function on the maximal torus $T \subset G$, the character is given by the ratio

$$
\operatorname{ch}_{R}\left(\mathrm{e}^{\phi}\right)=\frac{\mathrm{A}_{\alpha+\rho}(\phi)}{\mathrm{A}_{\rho}(\phi)}, \quad \mathrm{e}^{\phi} \in T .
$$

As throughout, $\alpha$ is the highest weight of $R$, and $\rho$ is the Weyl vector (7.27) given by half the sum of the positive roots of $G$.

Though we will not prove the character formula here, let us make a few basic comments about it. First, because $\mathrm{A}_{\alpha}$ is alternating under $\mathfrak{W}$, the ratio appearing on the right of (7.144) is invariant under $\mathfrak{W}$, as required of any character when restricted to $T$. Second, because $\rho$ is a weight of $G$ (a slightly non-trivial fact), the expression for $\operatorname{ch}_{R}$ in (7.144) is welldefined as a function on $T$, just as for the discussion surrounding (4.29). Finally, the character formula in (7.144) is manifestly correct in the special case $\alpha=0$, for which $R$ is trivial and $\operatorname{ch}_{R}=1$. Also, when $G=S U(2)$ and $\mathfrak{W}=\mathbb{Z}_{2}$ acting by reflection, one can readily check that (7.144) reproduces the formula for $\mathrm{ch}_{\mathbf{j}}$ in (7.61) with a suitable choice of coordinate on $\mathfrak{t} \cong \mathbb{R}$.

Let us now interpret our result (7.141) for $\left.Z(\epsilon ; C, R)\right|_{\mathcal{O}_{\alpha} / G}$ in light of the character formula. This interpretation is slightly more straightforward when $\alpha$ is a regular weight of $G$, so we specialize to the regular case first.

When $\alpha$ is regular, $G_{\alpha}=T,\left|\mathfrak{W}_{\alpha}\right|=1$, and $\rho^{[\alpha]}$ reduces to the Weyl vector $\rho$ itself. Also, the product over roots $\beta_{+}$satisfying $\left(\beta_{+}, \alpha\right)>0$ in (7.141) is simply the product over all positive roots $\beta>0$ of $G$. As a result, the final factor in the integrand of (7.141) reduces to the Weyl denominator $\mathrm{A}_{\rho}$,

$$
\mathrm{A}_{\rho}(\phi)=\prod_{\beta>0} 2 \sinh \left(\frac{\langle\beta, \phi\rangle}{2}\right) .
$$


See Lemma 24.3 of [52] for a proof of this well-known product formula for the Weyl denominator. Thus for regular weights,

$$
\begin{aligned}
\left.Z(\epsilon ; C, R)\right|_{\mathcal{O}_{\alpha} / G} \\
=\exp \left[-\frac{\mathrm{i} \pi}{2} \eta_{0}(0)\right] \frac{1}{\operatorname{Vol}(T)}\left(\frac{1}{\mathrm{i} \sqrt{\mathrm{P}}}\right)^{\Delta_{T}} \\
\quad \times \int_{\mathfrak{t} \times \mathcal{C}^{(0)}}[d \phi] \frac{1}{\mathrm{~A}_{\rho}(\phi)} \exp \left[-\langle\alpha+\rho, \phi\rangle-\frac{\mathrm{i}}{2 \epsilon_{\mathrm{r}}}\left(\frac{d}{\mathrm{P}}\right) \operatorname{Tr}\left(\phi^{2}\right)\right] \\
\quad \times \prod_{\beta>0}\left[2 \sinh \left(\frac{\langle\beta, \phi\rangle}{2}\right)\right]^{2-N} \prod_{j=1}^{N}\left[2 \sinh \left(\frac{\langle\beta, \phi\rangle}{2 a_{j}}\right)\right], \quad \alpha \text { regular. }
\end{aligned}
$$

To simplify (7.146) further, we make an elementary observation regarding discrete symmetries. By definition, the measure $[d \phi]$ in the contour integral is invariant under the Weyl group $\mathfrak{W}$ of $G$. Moreover, the integrand of (7.146) can generally be decomposed as a sum of terms, each of which transforms in a one-dimensional representation of $\mathfrak{W}$. Then since $[d \phi]$ is Weyl invariant, only the Weyl invariant piece of the integrand actually contributes to the integral over $\phi$.

Now, of the various factors in that integrand, the quadratic function $\operatorname{Tr}\left(\phi^{2}\right)$ appearing in the argument of the exponential in (7.146) is obviously Weyl invariant. Since $\mathfrak{W}$ is generated by reflections in the root lattice of $G$, the expression in the last line of (7.146) is also Weyl invariant, as it arises from a product over all positive roots $\beta>0$ of the even function

$$
\begin{aligned}
& F_{\beta}(\phi)=\left[2 \sinh \left(\frac{\langle\beta, \phi\rangle}{2}\right)\right]^{2-N} \prod_{j=1}^{N}\left[2 \sinh \left(\frac{\langle\beta, \phi\rangle}{2 a_{j}}\right)\right], \\
& F_{\beta}(\phi)=F_{\beta}(-\phi)=F_{-\beta}(\phi) .
\end{aligned}
$$

So in the integrand of (7.146), we are left to consider the factor

$$
S_{\alpha}(\phi)=\frac{\mathrm{e}^{-\langle\alpha+\rho, \phi\rangle}}{\mathrm{A}_{\rho}(\phi)} .
$$

By construction, the Weyl denominator $\mathrm{A}_{\rho}(\phi)$ is alternating under $\mathfrak{W}$. Therefore, only the alternating piece of the numerator $\exp [-\langle\alpha+\rho, \phi\rangle]$ in $S_{\alpha}(\phi)$ actually contributes to the contour integral over $\phi$ in (7.146). We 
immediately recognize that alternating piece to be

$$
\begin{aligned}
\mathrm{A}\left[\mathrm{e}^{-\langle\alpha+\rho, \phi\rangle}\right] & \equiv \frac{1}{|\mathfrak{W}|} \sum_{w \in \mathfrak{W}}(-1)^{w} \mathrm{e}^{\langle w \cdot(\alpha+\rho),-\phi\rangle}, \\
& =\frac{1}{|\mathfrak{W}|} \mathrm{A}_{\alpha+\rho}(-\phi), \\
& =(-1)^{\left(\Delta_{G}-\Delta_{T}\right) / 2} \cdot \frac{1}{|\mathfrak{W}|} \mathrm{A}_{\alpha+\rho}(\phi) .
\end{aligned}
$$

Here $\mathrm{A}[\cdot]$ denotes anti-symmetrization under $\mathfrak{W}$, and we have been careful to divide by the order of $\mathfrak{W}$ to ensure that the anti-symmetrization in (7.149) is properly normalized. Also, the overall sign $(-1)^{\left(\Delta_{G}-\Delta_{T}\right) / 2}$ in the last line of (7.149) accounts for reflecting $-\phi$ to $\phi$ in the argument of $\mathrm{A}_{\alpha+\rho}$, just as in $(7.143) .{ }^{36}$

Without loss, we replace $S_{\alpha}(\phi)$ in the integrand of (7.146) with the Weylinvariant function

$$
S_{\alpha}(\phi) \stackrel{[\cdot]^{\mathfrak{W}}}{\longmapsto} \frac{(-1)^{\left(\Delta_{G}-\Delta_{T}\right) / 2}}{|\mathfrak{W}|} \cdot \frac{\mathrm{A}_{\alpha+\rho}(\phi)}{\mathrm{A}_{\rho}(\phi)} .
$$

Via the character formula in (7.144), we finally obtain the following elegant result for the contribution of $\left\{\varrho_{\mathrm{ab}}\right\} \cong \mathcal{O}_{\alpha} / G$ to the Seifert loop path integral,

$$
\begin{aligned}
\left.Z(\epsilon ; C, R)\right|_{\mathcal{O}_{\alpha} / G} \\
=\exp \left[-\frac{\mathrm{i} \pi}{2} \eta_{0}(0)\right] \frac{1}{|\mathfrak{W}|} \frac{(-1)^{\left(\Delta_{G}-\Delta_{T}\right) / 2}}{\operatorname{Vol}(T)}\left(\frac{1}{\mathrm{i} \sqrt{\mathrm{P}}}\right)^{\Delta_{T}} \\
\quad \times \int_{\mathfrak{t} \times \mathcal{C}^{(0)}}[d \phi] \operatorname{ch}_{R}\left(\mathrm{e}^{\phi}\right) \exp \left[-\frac{\mathrm{i}}{2 \epsilon_{\mathrm{r}}}\left(\frac{d}{\mathrm{P}}\right) \operatorname{Tr}\left(\phi^{2}\right)\right] \\
\quad \times \prod_{\beta>0}\left[2 \sinh \left(\frac{\langle\beta, \phi\rangle}{2}\right)\right]^{2-N} \prod_{j=1}^{N}\left[2 \sinh \left(\frac{\langle\beta, \phi\rangle}{2 a_{j}}\right)\right], \quad \alpha \text { regular. }
\end{aligned}
$$

As claimed, all dependence on the weight $\alpha$ has been subsumed into the character $\mathrm{ch}_{R}$, which represents the Seifert loop operator under localization on $\mathcal{O}_{\alpha} / G$.

\footnotetext{
${ }^{36}$ The same sign under reflection can be seen directly, for instance, in the Weyl denominator formula (7.145).
} 
"ATMP-17-1-A1-BEA" — 2013/5/15 — 19:32 — page 166 — \#166

\subsubsection{A remark on equivariant pushdown}

Before we proceed further, let us make one theoretical remark about the interpretation of the Seifert loop operator as the character $\mathrm{ch}_{R}$. Although this remark is not strictly necessary, it nicely foreshadows certain aspects of the computation that we will perform in Section 7.3.

As in (7.36), we consider the distinguished abelian connection $\left\{\varrho_{\mathrm{ab}}\right\} \cong$ $\mathcal{O}_{\alpha} / G$ to fiber equivariantly over the trivial connection $\{0\}=p t / G$,

$$
\begin{gathered}
\mathcal{O}_{\alpha} / G \\
\underset{p t}{\downarrow} \mathrm{q} \\
p t G
\end{gathered} .
$$

When $\alpha$ is regular, localization on $\mathcal{O}_{\alpha}=G / T$ naturally produces an element of the equivariant cohomology ring

$$
H_{G}^{*}\left(\mathcal{O}_{\alpha}\right)=H_{G}^{*}(G / T)=H_{T}^{*}(p t),
$$

which we recognize to be the ring of functions on the Cartan subalgebra $\mathfrak{t} \subset \mathfrak{g}$. Indeed, the particular function on $\mathfrak{t}$, which we obtain via localization is precisely the integrand of (7.146). Given the equivariant fibration in (7.152), we can then push the result of localization on $\left\{\varrho_{\mathrm{ab}}\right\} \in \mathcal{M}(C, \alpha)$ down to the point $\{0\} \in \mathcal{M}$. But what does this equivariant pushdown mean?

Just as in (7.153), localization at the point $\{0\}$ naturally produces an element of $H_{G}^{*}(p t)$, which is the ring of invariant functions on the Lie algebra $\mathfrak{g}$. As well-known, the ring of invariant functions on $\mathfrak{g}$ is isomorphic to the ring of Weyl-invariant functions on $\mathfrak{t}$, so that

$$
H_{G}^{*}(p t) \cong H_{T}^{*}(p t)^{\mathfrak{W}} .
$$

The pushdown $\mathrm{q}_{*}: H_{G}^{*}\left(\mathcal{O}_{\alpha}\right) \rightarrow H_{G}^{*}(p t)$ is then simply symmetrization under $\mathfrak{W}$, exactly as in (7.150). So from the theoretical perspective, the character $\mathrm{ch}_{R}$ that represents the Seifert loop operator in (7.151) should not really be interpreted as the result of localization at the point $\left\{\varrho_{\mathrm{ab}}\right\} \in \mathcal{M}(C, \alpha)$, but rather as the result of localization at $\left\{\varrho_{\mathrm{ab}}\right\}$ followed by equivariant pushdown to $\{0\} \in \mathcal{M}$.

\subsubsection{Extension to irregular weights}

So far, we have assumed the weight $\alpha>0$ to be regular. For instance, if $G=S U(2)$, then all non-zero weights are regular, and (7.151) reproduces 
the empirical result in (7.64), at least up to an overall phase which we discuss at the end of this section.

However, for groups other than $S U(2)$, some weights are inevitably irregular. As an elementary example, the weight associated to the fundamental $(r+1)$-dimensional representation of $S U(r+1)$ is not regular when $r>1$. We certainly want to consider Wilson loops such as those associated to the fundamental representation of $S U(r+1)$, so let us quickly consider what happens to the preceding analysis when $\alpha$ is an irregular weight.

To start, we decompose the roots $\beta$ of $G$ into two sets, consisting of roots $\beta_{+}$for which $\left(\beta_{+}, \alpha\right) \neq 0$ and roots $\beta_{\perp}$ for which $\left(\beta_{\perp}, \alpha\right)=0$, just as in (7.138). The set of roots $\beta_{\perp}$ is empty when $\alpha$ is regular, and the set of roots $\beta_{\perp}$ runs over all roots when $\alpha$ vanishes. The Weyl denominator $\mathrm{A}_{\rho}$ in (7.145) then factorizes as a product over each set,

$$
\mathrm{A}_{\rho}(\phi)=\left[\prod_{\left(\beta_{+}, \alpha\right)>0} 2 \sinh \left(\frac{\left\langle\beta_{+}, \phi\right\rangle}{2}\right)\right] \cdot\left[\prod_{\beta_{\perp}>0} 2 \sinh \left(\frac{\left\langle\beta_{\perp}, \phi\right\rangle}{2}\right)\right] .
$$

Using (7.155), we rewrite the general contour integral in (7.141) as

$$
\begin{aligned}
\left.Z(\epsilon ; C, R)\right|_{\mathcal{O}_{\alpha} / G} \\
=\exp \left[-\frac{\mathrm{i} \pi}{2} \eta_{0}(0)\right] \frac{1}{\left|\mathfrak{W}_{\alpha}\right|} \frac{(-1)^{\left(\Delta_{G_{\alpha}}-\Delta_{T}\right) / 2}}{\operatorname{Vol}(T)}\left(\frac{1}{\mathrm{i} \sqrt{\mathrm{P}}}\right)^{\Delta_{T}} \\
\quad \times \int_{\mathfrak{t} \times \mathcal{C}^{(0)}}[d \phi] \frac{1}{\mathrm{~A}_{\rho}(\phi)}\left[\prod_{\beta_{\perp}>0} 2 \sinh \left(\frac{\left\langle\beta_{\perp}, \phi\right\rangle}{2}\right)\right] \exp \left[-\left\langle\alpha+\rho^{[\alpha]}, \phi\right\rangle\right] \\
\quad \times \exp \left[-\frac{\mathrm{i}}{2 \epsilon_{\mathrm{r}}}\left(\frac{d}{\mathrm{P}}\right) \operatorname{Tr}\left(\phi^{2}\right)\right] \prod_{\beta>0}\left[2 \sinh \left(\frac{\langle\beta, \phi\rangle}{2}\right)\right]^{2-N} \\
\quad \times \prod_{j=1}^{N}\left[2 \sinh \left(\frac{\langle\beta, \phi\rangle}{2 a_{j}}\right)\right]
\end{aligned}
$$

Once again, we wish to tease the character $\operatorname{ch}_{R}$ out of the integrand in (7.156). To do so, let us introduce the following function of $\phi$,

$$
\mathrm{B}_{\alpha}(\phi)=\mathrm{e}^{\left\langle\alpha+\rho^{[\alpha]}, \phi\right\rangle} \cdot \prod_{\beta_{\perp}>0}\left[2 \sinh \left(\frac{\left\langle\beta_{\perp}, \phi\right\rangle}{2}\right)\right],
$$


in terms of which we write the factor in the second line of (7.156) as

$$
\begin{aligned}
S_{\alpha}(\phi) & =\frac{\mathrm{e}^{-\left\langle\alpha+\rho^{[\alpha]}, \phi\right\rangle}}{\mathrm{A}_{\rho}(\phi)} \cdot\left[\prod_{\beta_{\perp}>0} 2 \sinh \left(\frac{\left\langle\beta_{\perp}, \phi\right\rangle}{2}\right)\right], \\
& =(-1)^{\left(\Delta_{G_{\alpha}}-\Delta_{T}\right) / 2} \cdot \frac{\mathrm{B}_{\alpha}(-\phi)}{\mathrm{A}_{\rho}(\phi)} .
\end{aligned}
$$

By the same symmetry argument as before, only the Weyl-invariant component of $S_{\alpha}(\phi)$, or equivalently the alternating component of $\mathrm{B}_{\alpha}(\phi)$, contributes to the contour integral over $\phi$.

The function $\mathrm{B}_{\alpha}(\phi)$ turns out to have some nice properties, which make evaluating its alternating component under $\mathfrak{W}$ particularly easy. For instance, in the extreme case that $\alpha$ vanishes, $\rho^{[\alpha]}$ vanishes as well, and the product over $\beta_{\perp}$ in (7.157) runs over all roots of $G$. Via the denominator formula in (7.145), $\mathrm{B}_{\alpha}(\phi)$ then reduces to $\mathrm{A}_{\rho}(\phi)$, as required for (7.156) to reproduce the contribution from the trivial connection on $M$ to Chern-Simons partition function.

More generally, $\mathrm{B}_{\alpha}(\phi)$ satisfies an identity, which extends the denominator formula in (7.145). According to this extended denominator formula, $\mathrm{B}_{\alpha}(\phi)$ can be rewritten as an alternating sum over elements $w^{\prime}$ of the Weyl group $\mathfrak{W}_{\alpha}$ of the stabilizer $G_{\alpha}$, so that

$$
\mathrm{B}_{\alpha}(\phi)=\sum_{w^{\prime} \in \mathfrak{W}_{\alpha}}(-1)^{w^{\prime}} \mathrm{e}^{\left\langle w^{\prime} \cdot(\alpha+\rho), \phi\right\rangle} .
$$

When $\alpha$ is regular, the Weyl group $\mathfrak{W}_{\alpha}$ is trivial, and the statement in (7.159) is immediate. Otherwise, $\mathfrak{W}_{\alpha}$ is non-trivial for irregular weights, in which case the identity in (7.159) has non-trivial content. Though we spare the reader the details, (7.159) can be proven in exactly the same way as the Weyl denominator formula, to which (7.159) reduces when $\alpha=0$. For sake of completeness, we sketch such a proof in Appendix D.

Given the identity in (7.159), the alternating component of $\mathrm{B}_{\alpha}(\phi)$ is easy to evaluate. Clearly,

$$
\begin{aligned}
\mathrm{A}\left[\mathrm{B}_{\alpha}(\phi)\right] & =\frac{1}{|\mathfrak{W}|} \sum_{w \in \mathfrak{W}}(-1)^{w} \mathrm{~B}_{\alpha}(w \cdot \phi), \\
& =\frac{1}{|\mathfrak{W}|} \sum_{w \in \mathfrak{W}} \sum_{w^{\prime} \in \mathfrak{W}_{\alpha}}(-1)^{\left(w w^{\prime}\right)} \mathrm{e}^{\left\langle\left(w w^{\prime}\right) \cdot(\alpha+\rho), \phi\right\rangle},
\end{aligned}
$$




$$
=\frac{1}{|\mathfrak{W}|} \sum_{w^{\prime} \in \mathfrak{W}_{\alpha}} \mathrm{A}_{\alpha+\rho}(\phi)=\frac{\left|\mathfrak{W}_{\alpha}\right|}{|\mathfrak{W}|} \mathrm{A}_{\alpha+\rho}(\phi) .
$$

In complete analogy to (7.150), we apply the identity in (7.160) to symmetrize $S_{\alpha}(\phi)$ under $\mathfrak{W}$,

$$
S_{\alpha}(\phi) \stackrel{[\cdot]^{\mathfrak{W}}}{\longmapsto}(-1)^{\left(\Delta_{G}-\Delta_{G_{\alpha}}\right) / 2} \cdot \frac{\left|\mathfrak{W}_{\alpha}\right|}{|\mathfrak{W}|} \cdot \frac{\mathrm{A}_{\alpha+\rho}(\phi)}{\mathrm{A}_{\rho}(\phi)} .
$$

The sign on the right in (7.161) again arises after a reflection from $-\phi$ to $\phi$ in the argument of $\mathrm{A}_{\alpha+\rho}$.

Via the character formula (7.144), the contour integral in (7.156) then becomes

$$
\begin{aligned}
\left.Z(\epsilon ; C, R)\right|_{\mathcal{O}_{\alpha} / G}= & \exp \left[-\frac{\mathrm{i} \pi}{2} \eta_{0}(0)\right] \frac{1}{|\mathfrak{W}|} \frac{(-1)^{\left(\Delta_{G}-\Delta_{T}\right) / 2}}{\operatorname{Vol}(T)}\left(\frac{1}{\mathrm{i} \sqrt{\mathrm{P}}}\right)^{\Delta_{T}} \\
& \times \int_{\mathfrak{t} \times \mathcal{C}^{(0)}}[d \phi] \operatorname{ch}_{R}\left(\mathrm{e}^{\phi}\right) \exp \left[-\frac{\mathrm{i}}{2 \epsilon_{\mathrm{r}}}\left(\frac{d}{\mathrm{P}}\right) \operatorname{Tr}\left(\phi^{2}\right)\right] \\
& \times \prod_{\beta>0}\left[2 \sinh \left(\frac{\langle\beta, \phi\rangle}{2}\right)\right]^{2-N} \prod_{j=1}^{N}\left[2 \sinh \left(\frac{\langle\beta, \phi\rangle}{2 a_{j}}\right)\right],
\end{aligned}
$$

exactly as in the regular case (7.151). So regardless of whether $\alpha$ is regular or irregular, the Seifert loop operator reduces to the character $\operatorname{ch}_{R}$ under localization on $\left\{\varrho_{\mathrm{ab}}\right\} \cong \mathcal{O}_{\alpha} / G$ and subsequent pushdown to the trivial connection $\{0\}$ on $M$.

\subsubsection{On framings and the phase of $Z(\epsilon ; C, R)$}

We finally wish to discuss the phase of our result (7.162) for the local contribution from $\left\{\varrho_{\mathrm{ab}}\right\} \cong \mathcal{O}_{\alpha} / G$ to $Z(\epsilon ; C, R)$. This discussion extends related remarks at the very end of Section 5.2 in [18] concerning the phase of the partition function $Z(\epsilon)$.

With no essential loss, we specialize to the case $G=S U(2)$, for which we can make a precise comparison between (7.162) and the result of Lawrence and Rozansky in (7.64). After parameterizing $\phi \in \mathfrak{t} \cong \mathbb{R}$ in terms of a coordinate $z$ via $\phi=(i / 2) \operatorname{diag}(z,-z)$, we easily see that the localization result in (7.162) agrees exactly with the corresponding empirical result in (7.64), at least up to a phase. However, the phases of the expressions in (7.162) and (7.64) do differ. 
To be precise, in the case $G=S U(2)$ with $R \cong \mathbf{j}$, the ratio $\exp (\mathrm{i} \delta \Psi)$ between the canonical phase in (7.64) and the phase in (7.162) is given by

$$
\begin{aligned}
\exp (\mathrm{i} \delta \Psi) & =\exp \left[\frac{\mathrm{i} \pi}{4}\left(\frac{k}{k+2}\right) \theta_{0}\right] \cdot \exp \left[\frac{-\mathrm{i} \pi}{2(k+2)}\left(j^{2}-1\right) \mathrm{P}\right] \\
\theta_{0} & =3-\frac{d}{\mathrm{P}}+12 \sum_{j=1}^{N} s\left(b_{j}, a_{j}\right) \\
\mathrm{P} & =\prod_{j=1}^{N} a_{j} \quad \text { if } N \geq 1, \quad \mathrm{P}=1 \quad \text { otherwise. }
\end{aligned}
$$

In computing $\delta \Psi$, we have used the expression (7.109) for $\eta_{0}(0)$ in the case that $M$ is a Seifert homology sphere, or a cyclic $\mathbb{Z}_{d}$ quotient thereof. Most significantly, we write $\exp (i \delta \Psi)$ in (7.163) as a product of two factors, the first proportional to $\theta_{0}$ and the second proportional to P. As we now explain, these factors are associated to the respective choices of framing for the Seifert manifold $M$ and the embedded curve $C \subset M$.

In general, under a shift in the framing of $M$ by $s$ units, the phase of $Z(\epsilon ; C, R)$ transforms as

$$
\begin{gathered}
Z(\epsilon ; C, R) \stackrel{s}{\longmapsto} \exp \left[\frac{\mathrm{i} \pi c}{12} s\right] \cdot Z(\epsilon ; C, R), \quad s \in \mathbb{Z}, \\
c=\frac{k \Delta_{G}}{k+\check{c}_{\mathfrak{g}}}, \quad \Delta_{G}=\operatorname{dim} G .
\end{gathered}
$$

Here, the constant $c$ is the central charge of the current algebra for $G$ at level $k$. Similarly, under a shift in the framing of $C$ by $t$ units, the phase of $Z(\epsilon ; C, R)$ transforms as

$$
\begin{aligned}
Z(\epsilon ; C, R) & \stackrel{t}{\longmapsto} \exp \left[2 \pi \mathrm{i} h_{R} t\right] \cdot Z(\epsilon ; C, R), \quad t \in \mathbb{Z}, \\
h_{R} & =\frac{(\alpha, \alpha+2 \rho)}{2\left(k+\check{c}_{\mathfrak{g}}\right)} .
\end{aligned}
$$

Here $h_{R}$ is the conformal weight of the current algebra primary associated to the representation $R .^{37}$

Because of the intrinsic ambiguity in the phase of $Z(\epsilon ; C, R)$ under shifts in the framing of the pair $(M, C)$, we need only check that the relative phase

\footnotetext{
${ }^{37}$ As throughout, $\alpha$ in $(7.165)$ is the highest weight of $R, \rho$ is the Weyl vector for $G$, $\check{c}_{\mathfrak{g}}$ is the dual Coxeter number, and $(\cdot, \cdot)=-\operatorname{Tr}(\cdot)$ is the invariant metric on $\mathfrak{g} \cong \mathfrak{g}^{*}$.
} 
$\exp (\mathrm{i} \delta \Psi)$ between the formulae in (7.64) and (7.162) can be absorbed with suitable choices of $s$ and $t$ in (7.164) and (7.165). For the case at hand, with $G=S U(2)$ and $R \cong \mathbf{j}$, the central charge $c$ and the weight $h_{R}$ become

$$
c=\frac{3 k}{k+2}, \quad h_{R}=\frac{j^{2}-1}{4(k+2)} .
$$

Comparing to (7.163), we see immediately that the relative phase takes the requisite form

$$
\exp (\mathrm{i} \delta \Psi)=\exp \left[\frac{\mathrm{i} \pi c}{12} s\right] \cdot \exp \left[2 \pi \mathrm{i} h_{R} t\right]
$$

provided we set

$$
s=\theta_{0}, \quad t=-\mathrm{P} .
$$

Although apparently resolving the phase discrepancy in (7.163), this observation begs two immediate questions. First, at no stage of the localization computation did we actually specify a framing for $(M, C)$. So how did we manage to produce a definite phase for $Z(\epsilon ; C, R)$ at all?

Second and perhaps more technically, although the parameter $\theta_{0}=3-d$ is integral when the Riemann surface $\Sigma$ at the base of $M$ is smooth, $\theta_{0}$ is typically only rational when $\Sigma$ carries a non-trivial orbifold structure. For instance, in the case of the Seifert fibration of $S^{3}$ over $\Sigma=\mathbb{W} \mathbb{C P}_{\mathbf{p}, \mathbf{q}}^{1}$, we computed $\theta_{0}$ in $(7.68)$ to be $\theta_{0}=(\mathbf{p} / \mathbf{q})+(\mathbf{q} / \mathbf{p})$, which is certainly not an integer in general. (When $d=\mathbf{p}=\mathbf{q}=1$, corresponding to the smooth, degree one fibration of $S^{3}$ over $\mathbb{C P}^{1}$, we note the trivial equality $\theta_{0}=3-d=\frac{\mathbf{p}}{\mathbf{q}}+\frac{\mathbf{q}}{\mathbf{p}}=2$.) So how are we to make sense of the shift (7.168) in framing by $s=\theta_{0}$ units when $\Sigma$ is an orbifold?

We address these questions in turn.

We largely answered the first question at the end of Section 5.2 in [18], for which the crucial observation is the following. If $M$ is a Seifert manifold equipped with a locally free $U(1)_{\mathrm{R}}$ action, then $M$ possesses a pair of distinguished "two-framings". By definition, a two-framing of $M$ is a trivialization of the direct sum $T M^{\oplus 2}=T M \oplus T M$ of two copies of the tangent bundle $T M$. By way of comparison, a framing of $M$ is just a trivialization of $T M$ itself. Like an honest framing, a two-framing of $M$ suffices to determine a definite phase for the Chern-Simons partition function $Z(\epsilon)$.

The first of the distinguished two-framings on $M$ was introduced by Atiyah [13] and is very well-known. The Atiyah two-framing $\tilde{\alpha}$ is defined 
"ATMP-17-1-A1-BEA" — 2013/5/15 — 19:32 — page 172 — \#172

for any compact, oriented three-manifold $M$, not necessarily Seifert, and is characterized up to homotopy by the following property: if $W$ is any oriented four-manifold with boundary $M$, then the signature $\sigma(W)$ of $W$ is given by $\sigma(W)=\frac{1}{6} p_{1}\left(T W^{\oplus 2}, \tilde{\alpha}\right)$, where $p_{1}\left(T W^{\oplus 2}, \tilde{\alpha}\right)$ is the relative Pontrjagin class computed using the boundary trivialization by $\tilde{\alpha}$. When $M$ is endowed with the Atiyah two-framing, the Chern-Simons partition function $Z(\epsilon)$ can always be presented with a canonical phase, as appears for instance in the empirical formulae for $Z(\epsilon)$ in $[74,81]$.

The second two-framing, introduced in [18], exists only when $M$ admits a Seifert fibration $\pi: M \rightarrow \Sigma$. Briefly, the Seifert two-framing $\tilde{\beta}$ is defined as follows. By assumption, $M$ is equipped with a nowhere-vanishing vector field $\mathrm{R}$ which generates the given action by $U(1)_{\mathrm{R}}$. Therefore $\mathrm{R}$ determines a rank-one subbundle $[\mathrm{R}]$ of $T M$. The Seifert manifold $M$ is also equipped with an invariant contact form $\kappa$, which satisfies $\langle\kappa, R\rangle=1$. Thus the kernel of $\kappa$ is complementary to $\mathrm{R}$ in each fiber of $T M$, and $T M$ decomposes as the direct $\operatorname{sum} T M \cong[\mathrm{R}] \oplus \operatorname{ker}(\kappa)$. Equivalently,

$$
T M^{\oplus 2} \cong[\mathrm{R}] \oplus[\mathrm{R}] \oplus \operatorname{ker}(\kappa) \oplus \operatorname{ker}(\kappa) .
$$

Via the isomorphism in (7.169), we obtain a trivialization of $T M^{\oplus 2}$ from any trivialization of the rank-four bundle $\operatorname{ker}(\kappa) \oplus \operatorname{ker}(\kappa)$. The essential step in the construction of the Seifert two-framing $\tilde{\beta}$ is then to show that a natural trivialization, unique up to homotopy, exists for $\operatorname{ker}(\kappa) \oplus \operatorname{ker}(\kappa)$. In a nutshell, $\operatorname{ker}(\kappa) \oplus \operatorname{ker}(\kappa)$ is the pullback of a corresponding bundle on $\Sigma$, and the bundle on $\Sigma$ admits a natural trivialization as a $\operatorname{Spin}(4)$-bundle for dimensional reasons - specifically, the vanishing of the homotopy groups $\pi_{i}(\operatorname{Spin}(4))$ for $i<3$.

For further details on the construction of $\tilde{\beta}$, we refer the interested reader to Section 5.2 of [18]. Later on, we will say a bit more about the Seifert two-framing in the special case of the fibration $\pi: S^{3} \rightarrow \mathbb{W}_{\mathbb{P}, \mathbf{q}}^{1}$.

On a general Seifert manifold $M$, the homotopy types of the respective Atiyah and Seifert two-framings are distinct, $\tilde{\alpha} \neq \tilde{\beta}$, leading to two different but equally natural ways to define a phase for the partition function $Z(\epsilon)$. As we mentioned, empirical results such as (7.64) are presented with the phase defined by the Atiyah two-framing $\tilde{\alpha}$. On the other hand, when $Z(\epsilon)$ is computed using localization, one might expect the result to appear with the phase determined by the Seifert two-framing $\tilde{\beta}$. At least when the base $\Sigma$ of $M$ is smooth, with no orbifold points, we directly checked this expectation in [18], where we verified that $s=\theta_{0}=3-d$ in (7.168) correctly accounts for the shift in framing from $\tilde{\alpha}$ to $\tilde{\beta}$. 
"ATMP-17-1-A1-BEA" — 2013/5/15 - 19:32 — page 173 — \#173

LOCALIZATION FOR WILSON LOOPS IN CHERN-SIMONS

At the end of this section, we reconsider the situation when $\Sigma$ is an orbifold. However, before we indulge in orbifold technicalities, let us discuss the framing of the knot $C \subset M$, which is the essentially new ingredient at present.

\subsubsection{The Seifert framing on $C$}

By definition, a framing of $C$ is given by the choice of a non-vanishing normal vector field along $C \subset M$. Such a normal vector field determines a new curve $C^{\prime}$ which is a small displacement of $C$ along the vector field. The pair $\left(C, C^{\prime}\right)$, along with the two-framing of $M$, then determines a definite phase for the Wilson loop path integral $Z(\epsilon ; C, R)$.

Most important here, if $C$ is an arbitrary curve embedded in an integral homology sphere $M$, then $C$ admits a canonical framing. This framing is fixed up to homotopy by the condition that $C$ and its displacement $C^{\prime}$ have zero linking number inside $M$,

$$
\operatorname{lk}\left(C, C^{\prime}\right)=0,
$$

where the linking number in a general homology sphere is defined by the homology class of $C^{\prime}$ in the complement of $C$ (or vice versa),

$$
\operatorname{lk}\left(C, C^{\prime}\right)=\left[C^{\prime}\right] \in H_{1}\left(M^{o} ; \mathbb{Z}\right) \cong \mathbb{Z}, \quad M^{o}=M-C .
$$

If $M$ is an integral homology sphere, $H_{1}\left(M^{o} ; \mathbb{Z}\right)$ is freely-generated by the meridian $\mathrm{m}$ of $C$, from which we obtain the essential isomorphism $H_{1}\left(M^{o} ; \mathbb{Z}\right) \cong \mathbb{Z}$ in $(7.171)$. In particular, when $C$ carries the canonical framing $(7.170)$, the class of $C^{\prime}$ is trivial in $H_{1}\left(M^{o} ; \mathbb{Z}\right)$.

The canonical framing (7.170) of $C$ is roughly analogous to the Atiyah two-framing $\tilde{\alpha}$ of $M$, insofar as both are defined whether or not the pair $(M, C)$ is Seifert. On the other hand, when $M$ is Seifert and $C \subset M$ is a generic Seifert fiber, we have the option to introduce a homotopically distinct Seifert framing on $C$ analogous to the Seifert two-framing $\tilde{\beta}$ on $M$. Up to homotopy, the Seifert framing on $C$ is uniquely characterized by the condition that it be invariant under the Seifert $U(1)_{R}$ action. Such an invariant framing on $C$ clearly exists, but for sake of completeness we spell out its construction explicitly.

To construct the Seifert framing, we first pick a basepoint on $C$ and a nonvanishing normal vector $\nu_{0} \neq 0$ in the fiber of the normal bundle $N_{C / M}$ at that point. To be concrete, let us parameterize $C$ with a periodic coordinate $\tau \sim \tau+1$, so that $\tau=0$ corresponds to the basepoint at which $\nu_{0}$ is defined. 
"ATMP-17-1-A1-BEA" — 2013/5/15 — 19:32 — page 174 — \#174

By assumption, $C$ is a generic orbit of $U(1)_{R}$, which therefore acts freely on a neighborhood of $C$ in $M$. In particular, each point of $C$ is associated to a unique element of $U(1)_{\mathrm{R}}$, and we are welcome to use the periodic coordinate $\tau$ on $C$ to parameterize the group $U(1)_{\mathrm{R}}$ itself. In this way we obtain a family of maps $f^{\tau}: M \rightarrow M$, parameterized by $\tau \in[0,1)$, which describe the action of $U(1)_{\mathrm{R}}$ on $M$. Each map $f^{\tau}$ automatically induces a pushforward $f_{*}^{\tau}: T M \rightarrow T M$ on the tangent bundle $T M$. Since each $f^{\tau}$ also preserves the orbit $C$, we obtain by restriction a pushforward $\left.f_{*}^{\tau}\right|_{C}: T C \rightarrow T C$ acting on tangent vectors to $C$ itself. Hence $f^{\tau}$ also induces a pushforward $\left.f_{*}^{\tau}\right|_{C}: N_{C / M} \rightarrow N_{C / M}$ on the normal bundle $N_{C / M}$ associated to the embedding $C \subset M$.

We now use these pushforward maps on $N_{C / M}$ to extend the normal vector $\nu_{0}$ defined at the basepoint $\tau=0$ to an invariant, non-vanishing normal vector field $\nu_{\mathrm{R}}$ defined everywhere on $C$. To define $\nu_{\mathrm{R}}$ for all $\tau$, we simply set $\nu_{\mathrm{R}}(\tau)=\left.f_{*}^{\tau}\right|_{C}\left(\nu_{0}\right)$. By construction, $\nu_{\mathrm{R}}$ is invariant under the action of $U(1)_{R}$ on $C$. Since each $f_{*}^{\tau}$ is a fiberwise isomorphism in a neighborhood of $C \subset M$, the normal vector $\nu_{\mathrm{R}}(\tau)$ is also non-vanishing for all $\tau$. Finally, the only choices in our construction of $\nu_{\mathrm{R}}$ were the choices of $\nu_{0}$ and the parameter $\tau$, so the Seifert framing of $C$ is unique up to homotopy.

The Seifert framing on $C$ is defined for any Seifert manifold $M$, whether or not $M$ is a homology sphere. Consequently, using the Seifert two-framing on $M$ and the Seifert framing on $C$, we can always present the Seifert loop path integral $Z(\epsilon ; C, R)$ with a canonical phase, which we naturally expect to appear in computations of $Z(\epsilon ; C, R)$ by non-abelian localization.

As a check, let us compare the Seifert framing of $C$ embedded in an integral homology sphere $M$ to the canonical framing in (7.170), for which $C$ and its displacement $C^{\prime}$ have zero linking number. In the case of the Seifert framing, both $C$ and the normal vector field $\nu_{\mathrm{R}}$ are invariant under $U(1)_{\mathrm{R}}$, so the displacement $C_{\mathrm{R}}^{\prime}$ of $C$ along $\nu_{\mathrm{R}}$ is naturally invariant as well. Thus like $C$ itself, $C_{\mathrm{R}}^{\prime}$ is a Seifert fiber of $M$.

In general, the linking number of two Seifert fibers in $M$ is non-zero. Hence, the canonical framing in (7.170) is related to the Seifert framing by a shift of $t=\operatorname{lk}\left(C, C_{\mathrm{R}}^{\prime}\right)$ units. To make a quantitative comparison between the canonical and the Seifert framings, we are left to compute the linking number $\operatorname{lk}\left(C, C_{\mathrm{R}}^{\prime}\right)$ for two Seifert fibers of $M$.

According to the definition in (7.171), the linking number $\operatorname{lk}\left(C, C_{\mathrm{R}}^{\prime}\right)$ is given by the homology class of $C_{\mathrm{R}}^{\prime}$ in the complement $M^{o}=M-C$, where the class of $C_{\mathrm{R}}^{\prime}$ is to be measured as an appropriate multiple of the class of the meridian $\mathrm{m}$ of $C$. Of course, $H_{1}\left(M^{o} ; \mathbb{Z}\right)$ is the abelianization of $\pi_{1}\left(M^{o}\right)$, 
and in (7.34) we have already provided an explicit description of $\pi_{1}\left(M^{\circ}\right)$ via generators $\left\{\mathrm{c}_{j}, \mathrm{f}, \mathrm{m}\right\}, j=1, \ldots, N$, and relations

$$
\begin{aligned}
{[\mathrm{m}, \mathrm{f}] } & =\left[\mathrm{c}_{j}, \mathrm{f}\right]=1, \quad j=1, \ldots, N, \\
\mathrm{c}_{j}^{a_{j}} \mathrm{f}^{b_{j}} & =1, \\
\prod_{j=1}^{N} \mathrm{c}_{j} & =\mathrm{m} \mathrm{f}^{n} .
\end{aligned}
$$

In interpreting $(7.172)$, we recall that $f$ corresponds to the Seifert fiber of $M$, so we identify $C_{\mathrm{R}}^{\prime}=\mathrm{f}$.

Abelianizing the relations in (7.172), we directly obtain

$$
\mathrm{f}=\mathrm{m}^{-\mathrm{P}}, \quad \mathrm{P}=\prod_{j=1}^{N} a_{j}=\left[n+\sum_{j=1}^{N} \frac{b_{j}}{a_{j}}\right]^{-1} .
$$

In the latter formula for $\mathrm{P}$, we use the arithmetic condition (7.30) satisfied by the Seifert invariants of the integral homology sphere $M$. As a relation between homology classes $[\mathrm{f}]=\left[C_{\mathrm{R}}^{\prime}\right]$ and $[\mathrm{m}]$, the identity in $(7.173)$ is perhaps better written additively as

$$
[\mathrm{f}]=\left[C_{\mathrm{R}}^{\prime}\right]=-\mathrm{P} \cdot[\mathrm{m}] \in H_{1}\left(M^{o} ; \mathbb{Z}\right) .
$$

Consequently,

$$
t=\operatorname{lk}\left(C, C_{\mathrm{R}}^{\prime}\right)=-\mathrm{P},
$$

exactly as we found previously in (7.168).

\subsubsection{Further remarks on the Seifert two-framing}

To complete our analysis of phases and framings, we finally consider the potentially fractional assignment $s=\theta_{0}$ in (7.168), relevant when the base $\Sigma$ of $M$ is an orbifold. As a first step, let us elaborate slightly upon the paradox posed by this result.

We recall that a framing of $M$ is a trivialization of $T M$. Concretely, such a trivialization is given by three smooth, non-vanishing vector fields which provide an oriented basis for the tangent space at each point of $M$. Any two such framings are related by a smooth map $h: M \rightarrow S O(3)$, which takes one trivialization pointwise to the other. Up to continuous deformations, the difference between the given framings is then measured by the homotopy class of the map $h$. 
"ATMP-17-1-A1-BEA" — 2013/5/15 — 19:32 — page 176 — \#176

We actually want to consider not framings but two-framings, which are given by trivializations of the sum $T M^{\oplus 2}=T M \oplus T M$. The bundle $T M^{\oplus 2}$ carries a natural spin structure, induced from the diagonal embedding $S O(3) \subset S O(3) \times S O(3) \subset S O(6)$, and any two trivializations of $T M^{\oplus 2}$ as a Spin(6)-bundle are related by a smooth map $h: M \rightarrow \operatorname{Spin}(6)$. Again, up to continuous deformations, the difference between the given two-framings as trivializations of $T M^{\oplus 2}$ is measured by the homotopy class of the $\operatorname{map} h$.

By standard arguments, the homotopy class of $h$ takes values in $\pi_{3}$ $(\operatorname{Spin}(6)) \cong \mathbb{Z}$. We naturally want to identify the parameter $s=\theta_{0}$ in (7.168) with the class $[h] \in \pi_{3}(\operatorname{Spin}(6))$ which measures the difference between the Atiyah two-framing $\tilde{\alpha}$ and the Seifert two-framing $\tilde{\beta}$ on $M$. If the base $\Sigma$ of $M$ is a smooth Riemann surface, then $\theta_{0}=3-d$ is indeed an integer, and we have already checked in Section 5.2 of [18] that the identification $\theta_{0}=[h]$ is correct. But if $\theta_{0}$ is fractional, as can happen when $\Sigma$ has orbifold points, then $s=\theta_{0}$ cannot correspond to the homotopy class of any smooth map $h: M \rightarrow \operatorname{Spin}(6)$.

This paradox has a natural resolution: the Seifert two-framing $\tilde{\beta}$ is not necessarily smooth when $\Sigma$ is an orbifold. By this statement, we mean that the vector fields on $M$ which describe the trivialization of $T M^{\oplus 2}$ associated to $\tilde{\beta}$ are not themselves smooth but have singularities along the exceptional fibers of $M$ which sit over orbifold points of $\Sigma$. As a result, the map $h: M \rightarrow \operatorname{Spin}(6)$ relating the smooth, Atiyah two-framing $\tilde{\alpha}$ to the Seifert two-framing $\tilde{\beta}$ also has singularities, and the class $[h]$ need not be integral. Apparently, despite the orbifold singularities that may occur along exceptional fibers in $M$, the Seifert two-framing $\tilde{\beta}$ still suffices to fix the phase of $Z(\epsilon ; C, R)$, as we will see in the example below.

According to our earlier sketch, the construction of $\tilde{\beta}$ relies upon the existence of a natural trivialization for a certain $\operatorname{Spin}(4)$-bundle over $\Sigma$. When $\Sigma$ has orbifold points, the presence of orbifold singularities in the trivialization over $\Sigma$ is perhaps not terribly surprising. Nonetheless, to illustrate precisely the relation between orbifold points of $\Sigma$ and singularities of $\tilde{\beta}$, let us analyze the Seifert two-framing for the very simple Seifert fibration $\pi: S^{3} \rightarrow \mathbb{W} \mathbb{C P}_{\mathbf{p}, \mathbf{q}}^{1}$.

We recall that the parameter $s=\theta_{0}$ for the Seifert fibration of $S^{3}$ over $\Sigma=\mathbb{W} \mathbb{C P}_{\mathbf{p}, \mathbf{q}}^{1}$ is given by

$$
\theta_{0}=\frac{\mathbf{p}}{\mathbf{q}}+\frac{\mathbf{q}}{\mathbf{p}}, \quad \operatorname{gcd}(\mathbf{p}, \mathbf{q})=1
$$


So $\theta_{0}$ is fractional in all but the special case $\mathbf{p}=\mathbf{q}=1$, for which $\Sigma=\mathbb{C P}^{1}$ is smooth. Beyond merely establishing that $\tilde{\beta}$ is singular whenever $\theta_{0}$ is fractional, we would also like to understand why $\theta_{0}$ takes the particular value in (7.176).

One might worry that the quantitative, technical analysis of singular twoframings on even a three-manifold as simple as $S^{3}$ could become rather involved. To sidestep many potential complications arising from singularities in $\tilde{\beta}$, we follow a slightly indirect approach, adapted to the presentation of $S^{3}$ via surgery on $S^{2} \times S^{1}$. Besides its concreteness, the analysis of $\tilde{\beta}$ via surgery also makes clear the relation between the fractions in (7.176) and the orbifold points of orders $\mathbf{p}$ and $\mathbf{q}$ in $\mathbb{W C P}_{\mathbf{p}, \mathbf{q}}^{1}$.

We first recall that $S^{3}=T^{\prime} \cup_{\delta} T$ can be presented topologically as a union of solid tori $T^{\prime}$ and $T$ which are glued together along their boundaries by a non-trivial element $\delta$ in the mapping class group $S L(2, \mathbb{Z})$ of the twotorus $S^{1} \times S^{1}$. As standard, $\delta$ acts on the lattice $H_{1}\left(S^{1} \times S^{1} ; \mathbb{Z}\right) \cong \mathbb{Z} \oplus \mathbb{Z}$; to describe $\delta$ as a matrix, we pick a basis $\{\mathrm{m}, l\}$ for this lattice as follows. We identify $S^{1} \times S^{1}$ with the boundary of $T=D^{2} \times S^{1}$, where $D^{2}$ is the unit disk in $\mathbb{R}^{2}$. The meridian $m$ is then given by the boundary of $D^{2}$, and the longitude $l$ is given by the $S^{1}$ fiber over any point on that boundary. Equivalently, if we parameterize $D^{2}$ with polar coordinates $(r, \varphi)$ and $S^{1}$ with a periodic coordinate $\tau \sim \tau+1$, the meridian $\mathrm{m}$ is parameterized by $\varphi$, and the longitude $l$ is parameterized by $\tau$.

If $\delta$ were trivial in $S L(2, \mathbb{Z})$, then $T^{\prime} \cup_{\delta} T$ would simply be the aforementioned product $S^{2} \times S^{1}$, where $S^{2}$ arises by gluing together the respective $D^{2}$ factors in $T$ and $T^{\prime}$. To obtain $S^{3}$ instead of $S^{2} \times S^{1}$, we take $\delta$ to have the form

$$
\delta=\mathbf{T}^{b} \cdot \mathbf{S} \cdot \mathbf{T}^{a}, \quad a, b \in \mathbb{Z} .
$$

Here, $\mathbf{S}$ and $\mathbf{T}$ are the standard generators of $S L(2, \mathbb{Z})$,

$$
\mathbf{S}=\left(\begin{array}{ll}
0 & -1 \\
1 & 0
\end{array}\right), \quad \mathbf{T}=\left(\begin{array}{ll}
1 & 1 \\
0 & 1
\end{array}\right)
$$

and $(a, b)$ are arbitrary integers (possibly zero).

As will be of use momentarily, let us review how the form of $\delta$ in (7.177) arises. We regard $S^{3}$ as the unit sphere embedded in $\mathbb{C}^{2}$, parameterized by complex coordinates $(X, Y)$ satisfying $|X|^{2}+|Y|^{2}=1$. In these coordinates, the solid tori $T$ and $T^{\prime}$ in the decomposition $S^{3}=T^{\prime} \cup_{\delta} T$ are given without 
"ATMP-17-1-A1-BEA" — 2013/5/15 — 19:32 — page 178 — \#178

loss by the respective subsets of $S^{3}$ below, ${ }^{38}$

$$
T=\left\{\left.(X, Y) \in S^{3}|| X\right|^{2} \leq \frac{1}{2}\right\}, \quad T^{\prime}=\left\{\left.(X, Y) \in S^{3}|| Y\right|^{2} \leq \frac{1}{2}\right\} .
$$

In terms of the topological identification $T=D^{2} \times S^{1}$, the disk $D^{2}$ is parameterized up to an overall rescaling by the complex variable $X$, and the $S^{1}$ fiber is parameterized by the phase of the non-vanishing complex variable $Y$. Thus on the boundary of $T$, where $|X|^{2}=|Y|^{2}=1 / 2$, the phase of $X$ is identified with $\varphi$, and the phase of $Y$ is identified with $\tau$. In the other solid torus $T^{\prime}$, the roles of $X$ and $Y$ are reversed. The exchange $X \leftrightarrow Y$ in passing from $T$ to $T^{\prime}$ is then accomplished by the generator $\mathbf{S}$ in (7.177).

Otherwise, the choice of the pair $(a, b)$ is related to the possibility to perform Dehn twists by $\mathbf{T} \in S L(2, \mathbb{Z})$ on the boundaries of $T$ and $T^{\prime}$. Our description of $\delta$ as a matrix relies upon the choice of the particular basis $\{m, l\}$ for $H_{1}(\partial T ; \mathbb{Z})$. The meridian $m$ is canonically determined, at least up to sign, as the generator of the kernel of the natural map from $H_{1}(\partial T ; \mathbb{Z}) \cong$ $\mathbb{Z} \oplus \mathbb{Z}$ to $H_{1}(T ; \mathbb{Z}) \cong \mathbb{Z}$. But any $\mathrm{l}^{\prime}=\mathbf{T}^{a}(\mathrm{l})=\mathrm{l}+a \cdot \mathrm{m}$ is equally good as a complementary basis element for $H_{1}(\partial T ; \mathbb{Z})$. As a result, the possible twists by $\mathbf{T}^{a}$ and $\mathbf{T}^{b}$ acting on the respective boundaries of $T$ and $T^{\prime}$ do not change the topology of the union $S^{3}=T^{\prime} \cup_{\delta} T$.

However, the parameters $a$ and $b$ are far from irrelevant, since these data determine a definite two-framing on $S^{3}$. As carefully explained for instance in [51], the solid tori $T$ and $T^{\prime}$ can each be endowed with a standard twoframing, which restricts on the boundary to the product two-framing determined by the vector fields $\partial / \partial \varphi$ and $\partial / \partial \tau$. These standard two-framings on $T$ and $T^{\prime}$ then determine a corresponding two-framing on $S^{3}=T^{\prime} \cup_{\delta} T$ after gluing by $\delta$.

We now wish to relate the two-framing on $S^{3}$ determined by $\delta$ to both the Atiyah two-framing $\tilde{\alpha}$ and the Seifert two-framing $\tilde{\beta}$.

The relation to the Atiyah two-framing $\tilde{\alpha}$ is well-known. Briefly, with the conventions in (7.177), the Atiyah two-framing $\tilde{\alpha}$ is described by any pair $(a, b)$ such that $a+b=0$. Else, each Dehn twist by $\mathbf{T}$ shifts the standard two-framing on the solid torus by one unit, and the gluing under $\delta$ generally produces the two-framing on $S^{3}$ which is shifted by a total $s=a+b$ units relative to $\tilde{\alpha}$. Once again, $s=[h]$ is the homotopy class of the map

\footnotetext{
${ }^{38}$ The choice of the symmetric bound for $|X|^{2}$ and $|Y|^{2}$ by $1 / 2$, as opposed to any other parameters $x$ and $1-x$ in the unit interval, is inessential.
} 
$h: M \rightarrow \operatorname{Spin}(6)$ which measures the difference between $\tilde{\alpha}$ and the twoframing induced by $\delta$. That said, throughout the present analysis, we will really only keep track of the magnitude of $s$, not the overall sign. Finally, as a small check that the overall shift by $s=a+b$ units is correct, we consider the case $b=-a$. Then $\delta=\mathbf{T}^{-a} \cdot \mathbf{S} \cdot \mathbf{T}^{a}$ is conjugate to $\mathbf{S}$ in $S L(2, \mathbb{Z})$, and the two-framing determined by $\delta$ necessarily agrees with the two-framing determined by $\mathbf{S}$ alone.

We are left to describe the Seifert two-framing $\tilde{\beta}$ similarly in terms of surgery under $\delta$. As before, $\tilde{\beta}$ is constructed as a trivialization of $T M^{\oplus 2}$ using the evident isomorphism

$$
T M^{\oplus 2} \cong[\mathrm{R}] \oplus[\mathrm{R}] \oplus \operatorname{ker}(\kappa) \oplus \operatorname{ker}(\kappa),
$$

along with a certain natural trivialization of the rank-four bundle $\operatorname{ker}(\kappa) \oplus$ $\operatorname{ker}(\kappa)$. At first glance, one might worry that a detailed knowledge of the vector fields on $S^{3}$ responsible for trivializing $\operatorname{ker}(\kappa) \oplus \operatorname{ker}(\kappa)$ would be necessary to describe $\tilde{\beta}$ via surgery. However, all we really need to know is that the trivialization of $T M^{\oplus 2}$ associated to $\tilde{\beta}$ involves the non-vanishing, Seifert vector field R. In the current example, if $S^{3}$ is parameterized by complex coordinates $(X, Y)$ satisfying $|X|^{2}+|Y|^{2}=1$, then $U(1)_{\mathrm{R}}$ acts on $X$ and $Y$ with respective charges $\mathbf{p}$ and $\mathbf{q}$. The generating vector field $\mathrm{R}$ is then given explicitly by

$$
\mathrm{R}=\frac{\mathrm{i}}{2}\left[\mathbf{p} X \frac{\partial}{\partial X}+\mathbf{q} Y \frac{\partial}{\partial Y}-\mathbf{p} \bar{X} \frac{\partial}{\partial \bar{X}}-\mathbf{q} \bar{Y} \frac{\partial}{\partial \bar{Y}}\right] .
$$

Upon restriction, $\tilde{\beta}$ determines a two-framing on each of the solid tori $T$ and $T^{\prime}$ specified as subsets of $S^{3}$ in (7.179). So to characterize $\tilde{\beta}$ under surgery, we need only compare these induced two-framings on $T$ and $T^{\prime}$ to the standard two-framing. We first perform the comparison on $T$; the comparison on $T^{\prime}$ is entirely similar.

As we mentioned earlier, the standard two-framing on $T=D^{2} \times S^{1}$ restricts on the boundary $\partial T=S^{1} \times S^{1}$ to the product two-framing by the non-vanishing vector fields $\{\partial / \partial \varphi, \partial / \partial \tau\}$ which generate rotations in each $S^{1}$ factor. Equivalently, the standard two-framing identifies twice the tangent bundle of $S^{1} \times S^{1}$ with the sum

$$
T\left(S^{1} \times S^{1}\right)^{\oplus 2} \stackrel{\text { std }}{\cong}\left[\frac{\partial}{\partial \varphi}\right] \oplus\left[\frac{\partial}{\partial \varphi}\right] \oplus\left[\frac{\partial}{\partial \tau}\right] \oplus\left[\frac{\partial}{\partial \tau}\right] .
$$

Just as in $(7.180),[\cdot]$ indicates the trivial line bundle generated by the given vector field. 
In contrast, if we restrict the Seifert two-framing $\tilde{\beta}$ to the boundary of $T$, we obtain a different trivialization, now involving the Seifert vector field R. Up to homotopy, the Seifert two-framing $\tilde{\beta}$ identifies twice the tangent bundle of $S^{1} \times S^{1}$ with the sum

$$
T\left(S^{1} \times S^{1}\right)^{\oplus 2} \stackrel{\tilde{\beta}}{\cong}\left[\mathrm{R}^{\perp}\right] \oplus\left[\mathrm{R}^{\perp}\right] \oplus[\mathrm{R}] \oplus[\mathrm{R}] .
$$

Here $\mathrm{R}^{\perp}$ is a non-vanishing vector field on $S^{1} \times S^{1}$ which generates the orthocomplement to $\mathrm{R}$ as determined by any convenient metric. (The homotopy class of the trivialization does not depend on the continuous choice of the metric on $S^{1} \times S^{1}$.)

Again up to homotopy, any two-framing on the solid torus $T$ is related to the standard two-framing by the repeated action of the Dehn twist $\mathbf{T}$ on the boundary. To relate the respective trivializations in (7.182) and (7.183), we recall that $\varphi$ is identified on $\partial T$ with the phase of the coordinate $X$, and $\tau$ is identified with the phase of the coordinate $Y$. Hence, $\mathrm{R}$ in (7.181) is given on the boundary of $T$ by

$$
\mathbf{R}=\mathbf{p} \frac{\partial}{\partial \varphi}+\mathbf{q} \frac{\partial}{\partial \tau}
$$

Further, with a suitable choice of metric on $\partial T$, we can always arrange that $\mathrm{R}^{\perp}=\partial / \partial \varphi$. The trivialization (7.183) induced by $\tilde{\beta}$ on $\partial T$ then becomes

$$
T\left(S^{1} \times S^{1}\right)^{\oplus 2} \stackrel{\tilde{\beta}}{\cong}\left[\frac{\partial}{\partial \varphi}\right] \oplus\left[\frac{\partial}{\partial \varphi}\right] \oplus\left[\frac{\partial}{\partial \tau}+\left(\frac{\mathbf{p}}{\mathbf{q}}\right) \frac{\partial}{\partial \varphi}\right] \oplus\left[\frac{\partial}{\partial \tau}+\left(\frac{\mathbf{p}}{\mathbf{q}}\right) \frac{\partial}{\partial \varphi}\right] .
$$

To facilitate the comparison between (7.182) and (7.185), we have rescaled $\mathrm{R}$ in (7.184) by an overall factor of $1 / \mathbf{q}$. Of course, such a scaling has no effect on the line bundle generated by $\mathrm{R}$.

Under a general twist by $\mathbf{T}^{a}$, the line bundles in the standard product trivialization (7.182) transform as

$$
\left[\frac{\partial}{\partial \varphi}\right] \stackrel{\mathbf{T}^{a}}{\longmapsto}\left[\frac{\partial}{\partial \varphi}\right], \quad\left[\frac{\partial}{\partial \tau}\right] \stackrel{\mathbf{T}^{a}}{\longmapsto}\left[\frac{\partial}{\partial \tau}+a \frac{\partial}{\partial \varphi}\right] .
$$

From (7.186), we see immediately that $\mathbf{T}^{a}$ for $a=\mathbf{p} / \mathbf{q}$ formally takes the standard two-framing in (7.182) to the induced Seifert two-framing in (7.185).

Now, if $\mathbf{p}=\mathbf{q}=1$, then $\mathbf{T}^{a}=\mathbf{T}$ is the usual Dehn twist, which takes one smooth two-framing on $T$ to another, shifted by one unit. Otherwise, for 
relatively-prime pairs $\mathbf{p}>\mathbf{q}>1$, the Seifert two-framing $\tilde{\beta}$ on $T$ is related to the standard two-framing via the fractional twist $\mathbf{T}^{\mathbf{p} / \mathbf{q}}$. Such a fractional Dehn twist does not really make sense on a smooth three-manifold, but it does make sense on a three-dimensional orbifold. Indeed, the exceptional fiber given by $X=0$ in $T$ sits precisely over the orbifold point of order $\mathbf{q}$ in the base of the fibration $\pi: S^{3} \rightarrow \mathbb{W}_{\mathbb{C P}_{\mathbf{p}, \mathbf{q}}^{1}}^{1}$, from which the denominator in the exponent $a=\mathbf{p} / \mathbf{q}$ arises. ${ }^{39}$ Clearly as promised, the fractional twist by $\mathbf{T}^{\mathbf{p} / \mathbf{q}}$ on the standard two-framing creates orbifold singularities in $\tilde{\beta}$ along the exceptional fiber.

On the complementary torus $T^{\prime}$, the same analysis applies after an exchange of the coordinates $X$ and $Y$. Equivalently, because $X$ and $Y$ are distinguished only by their charges under $U(1)_{R}$, we simply exchange $\mathbf{p}$ and $\mathbf{q}$. Thus a twist by $\mathbf{T}^{b}$ for $b=\mathbf{q} / \mathbf{p}$ takes the standard two-framing on $T^{\prime}$ to the induced Seifert two-framing $\tilde{\beta}$. In total, we deduce that the difference between the Seifert two-framing $\tilde{\beta}$ and the Atiyah two-framing $\tilde{\alpha}$ on the union $S^{3}=T^{\prime} \cup_{\delta} T$ is given by the sum

$$
s=a+b=\frac{\mathbf{p}}{\mathbf{q}}+\frac{\mathbf{q}}{\mathbf{p}}
$$

exactly as in (7.176).

\subsection{The Seifert loop operator as a Chern character}

To conclude our analysis of Chern-Simons gauge theory on a Seifert threemanifold $M$, we now extend our work in Section 7.2 to describe the cohomology class of the Seifert loop operator $W_{R}(C)$ on any smooth component $\mathcal{M}_{0}$ in the moduli space of flat connections on $M$. Though the discussion here will be self-contained, the results in this section also build upon those in Section 5.3 of [18].

Mostly as a means to streamline the exposition, let us immediately make a few simplifying assumptions. First, we assume throughout that $M$ is the total space of a circle bundle of degree $n \geq 1$ over a smooth (non-orbifold)

\footnotetext{
${ }^{39}$ Let us briefly check that $X=0$ sits over the orbifold point of order $\mathbf{q}$, as opposed to $\mathbf{p}$, in $\mathbb{W C P}_{\mathbf{p}, \mathbf{q}}^{1}$. Since $|Y| \neq 0$ in $T$, we can partially fix a slice for the action of $U(1)_{\mathbf{R}}$ by imposing the condition that $Y=|Y|$ be real and positive. But since $Y$ transforms with charge $\mathbf{q}>1$ under $U(1)_{\mathrm{R}}$, the condition $Y=|Y|$ still leaves unfixed a cyclic subgroup $\mathbb{Z}_{\mathbf{q}} \subset U(1)_{\mathrm{R}}$, whose generator acts on $X$ as $X \mapsto \zeta^{\mathbf{p}} \cdot X, \zeta=\exp (2 \pi \mathrm{i} / \mathbf{q})$. So under the quotient by $\mathbb{Z}_{\mathbf{q}}$, the point corresponding to $X=0$ in $\mathbb{W} \mathbb{C P}_{\mathbf{p}, \mathbf{q}}^{1}$ is an orbifold point of order q.
} 
Riemann surface $\Sigma$ of genus $h \geq 1$,

$$
S^{1} \stackrel{n}{\longrightarrow} \underset{\substack{\downarrow \\ \Sigma}}{M}, \quad \operatorname{genus}(\Sigma)=h \geq 1 .
$$

So unlike the examples in Section 7.2, for which orbifold points in genus zero were crucial, $\Sigma$ now has no orbifold points.

Second, because our current focus is on topology rather than group theory, we restrict attention to the familiar case that the Chern-Simons gauge group is $G=\mathrm{SU}(r+1)$. Similarly, for the irreducible representation $R$, we assume that the highest weight $\alpha>0$ of $R$ is regular. Hence, the stabilizer of $\alpha$ under the adjoint action is $G_{\alpha}=T=U(1)^{r}$, and the coadjoint orbit $\mathcal{O}_{\alpha} \cong G / T$ is the complete flag manifold. Explicitly, again using the identification $\mathfrak{t} \cong \mathfrak{t}^{*}$, we write

$$
\alpha=i \operatorname{diag}\left(\alpha_{1}, \ldots, \alpha_{r+1}\right), \quad \alpha_{1}+\cdots+\alpha_{r+1}=0,
$$

in terms of which the regularity condition $\alpha>0$ becomes

$$
\alpha_{1}>\alpha_{2}>\cdots>\alpha_{r}>\alpha_{r+1}
$$

Later, we will want to characterize the diagonal entries (7.189) of $\alpha$ in a slightly more invariant fashion. As in (5.55), we therefore introduce the basic generating weights $\left\{\hat{\omega}_{1}, \ldots, \hat{\omega}_{r+1}\right\}$ of $\mathrm{SU}(r+1)$, in terms of which $\alpha$ can be expanded as

$$
\alpha=\alpha_{1} \hat{\omega}_{1}+\cdots+\alpha_{r+1} \hat{\omega}_{r+1} .
$$

Finally, we assume without loss that the representation $R$ is integrable in the current algebra for $G$ at level $k$. This assumption implies that the highest weight $\alpha>0$ of $R$ is bounded in terms of the highest root $\vartheta$ of $G$ by $(\vartheta, \alpha)=\alpha_{1}-\alpha_{r+1} \leq k$. Actually, for technical reasons that will become clear, we need to assume that $\alpha$ satisfies the slightly stronger, strict bound $(\vartheta, \alpha)<k$. As we indicated in Section 7.1, the strict bound on $\alpha$ is conceptually the 'bare' version of the integrability condition expressed in the form $(\vartheta, \alpha+\rho)<k+\check{c}_{\mathfrak{g}}$.

In actuality, neither the restriction on $G$ nor $R$ is particularly essential, as exemplified by the preceding computation for general $(G, R)$ in Section 7.2. Moreover, the result we eventually obtain for the cohomology class which describes the Seifert loop operator $W_{R}(C)$ on $\mathcal{M}_{0}$ will have a natural meaning 
"ATMP-17-1-A1-BEA" — 2013/5/15 - 19:32 — page 183 — \#183

for arbitrary pairs $(G, R)$. Nonetheless, we prefer to phrase the discussion here in the language of holomorphic vector bundles, as opposed to bundles with arbitrary structure group, and we prefer to avoid the small complications, such as in the discussion surrounding (7.156), which arise when $\alpha$ is not regular.

The computation of the Seifert loop class on $\mathcal{M}_{0}$, through straightforward, is slightly involved. Rather than plunge into that computation straightaway, let us first present our eventual result, which is perhaps more illuminating than the computation itself. Indeed, the class in $H^{*}\left(\mathcal{M}_{0}\right)$ which describes the Seifert loop operator $W_{R}(C)$ under localization is not hard to guess, provided we note the following clues.

To start, the Seifert loop operator is defined solely by the choice of $R$ and the point $p \in \Sigma$ over which $C$ sits as a Seifert fiber of $M$, so the Seifert loop class can depend a priori only upon these data. Moreover, since the isotopy class of the embedding $C \subset M$ is invariant under continuous deformations of $p$, the Seifert loop class can really only depend upon the discrete choice of $R$.

Let us now ask - what are the natural cohomology classes to consider on $\mathcal{M}_{0}$ ?

The answer to this question is particularly clear if we recall from Section 7.1 the identification (7.20) of moduli spaces

$$
\mathcal{M}_{0} \cong \widetilde{\mathcal{N}}(P)
$$

where $\widetilde{\mathcal{N}}(P)$ is a finite, unramified cover of the moduli space $\mathcal{N}(P)$ parameterizing flat connections on a principal $G_{\text {ad }}(=G / \mathcal{Z}(G))$-bundle $P$ over $\Sigma$. Here the topology of $P$ is determined by the central fiberwise holonomy $\varrho(\mathrm{f}) \in \mathcal{Z}(G)$, constant for all points in $\mathcal{M}_{0}$, of the corresponding irreducible flat $G$-connection on $M$. For technical simplicity, we assume throughout that $\zeta=\varrho(\mathrm{f})^{n}$ in (7.18) generates the center $\mathcal{Z}(G)=\mathbb{Z}_{r+1}$, in which case $\mathcal{N}(P)$ is smooth, and the cover $\widetilde{\mathcal{N}}(P) \rightarrow \mathcal{N}(P)$ has degree $|\mathcal{Z}(G)|^{2 h}=(r+1)^{2 h}$ for $G=S U(r+1)$.

As in Section 5.2, when $\mathcal{N}(P)$ is smooth, a universal bundle $\mathcal{V}$ exists as a holomorphic vector bundle of rank $r+1$ over the product $\Sigma \times \mathcal{N}(P)$,

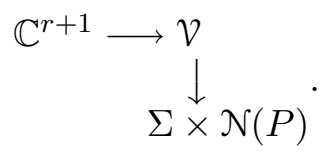


"ATMP-17-1-A1-BEA" — 2013/5/15 — 19:32 — page 184 — \#184

In this situation, the characteristic classes of $\mathcal{V}$ define natural cohomology classes on $\Sigma \times \mathcal{N}(P)$, and those characteristic classes can be evaluated on homology classes of $\Sigma$ to obtain associated classes on $\mathcal{N}(P)$ alone. From the perspective of gauge theory, these Atiyah-Bott classes (introduced in Section 9 of [12]) are the natural, God-given cohomology classes on $\mathcal{N}(P)$, and as demonstrated in Theorem 9.11 of [12], they suffice to generate multiplicatively the integral cohomology ring $H^{*}(\mathcal{N}(P) ; \mathbb{Z})$.

With this philosophy and the identification $\mathcal{M}_{0} \cong \widetilde{\mathcal{N}}(P)$, we see that the natural cohomology classes to consider on $\mathcal{M}_{0}$ are the pullbacks under the covering map $\widetilde{\mathcal{N}}(P) \rightarrow \mathcal{N}(P)$ of the Atiyah-Bott classes on $\mathcal{N}(P)$. To avoid cluttering the notation any further, we will not attempt to distinguish between cohomology classes on $\mathcal{N}(P)$ and their pullbacks to $\mathcal{M}_{0} \cong \widetilde{\mathcal{N}}(P)$.

As we mentioned above, each Atiyah-Bott class is defined by evaluating a given characteristic class of $\mathcal{V}$ on a given homology class of $\Sigma$. For the requisite homology class, we have already noted that the Seifert loop operator involves the choice of a point $p \in \Sigma$. The Atiyah-Bott classes on $\mathcal{N}(P)$ associated to the homology class of $p$ are then simply the characteristic classes of the bundle $\mathcal{V}$ restricted to $\{p\} \times \mathcal{N}(P)$,

$$
\left.\mathcal{V}_{p} \equiv \mathcal{V}\right|_{\{p\} \times \mathcal{N}(P)},
$$

as appeared already in (5.43).

We are finally left to guess which characteristic class of $\mathcal{V}_{p}$ actually describes the Seifert loop operator $W_{R}(C)$ under localization on $\mathcal{M}_{0}$. This characteristic class must somehow incorporate the choice of the representation $R$, for which there is a more-or-less evident way to proceed.

Via the Chern-Weil homomorphism, the characteristic classes of a bundle with structure group $G$ are determined by invariant functions on the Lie algebra $\mathfrak{g}$, or equivalently, by Weyl-invariant functions on the Cartan subalgebra $\mathfrak{t}$. When $M$ is a Seifert homology sphere, we have already demonstrated in Section 7.2 that the Seifert loop operator $W_{R}(C)$ reduces at the trivial connection $\{0\}=p t / G$ to the character $\operatorname{ch}_{R}$,

$$
\left.W_{R}(C)\right|_{\{0\}}=\operatorname{ch}_{R}\left(\mathrm{e}^{\phi}\right) \in H_{T}^{*}(p t)^{\mathfrak{W}}, \quad \phi \in \mathfrak{t},
$$

interpreted here as a Weyl-invariant function of $\phi \in \mathfrak{t}$. So for localization on $\mathcal{M}_{0} \cong \widetilde{\mathcal{N}}(P)$, the most natural - and certainly most elegant - possibility is that the Seifert loop operator $W_{R}(C)$ reduces to the characteristic class of 
$\mathcal{V}_{p}$ determined by the same character $\mathrm{ch}_{R}$,

$$
\left.W_{R}(C)\right|_{\mathcal{M}_{0}}=\operatorname{ch}_{R}\left(\mathcal{V}_{p}\right) \in H^{*}\left(\mathcal{M}_{0}\right)
$$

In the remainder of this paper, our goal will be to demonstrate the identification in (7.196) directly, by a localization computation on $\mathcal{M}_{0}$ very similar to the computation in Section 7.2. However, before we even begin to compute, let us first provide a more concrete description of the characteristic class $\operatorname{ch}_{R}\left(\mathcal{V}_{p}\right)$, as we will encounter it in practice.

In general, any characteristic class of holomorphic vector bundle can be expressed as a symmetric function of its Chern roots. With a certain malice aforethought, we were careful to provide a complete description of the Chern roots of $\mathcal{V}_{p}$ at the end of Section 5.2. As we reviewed there, the extended moduli space $\mathcal{N}(P ; \lambda)$ for regular $\lambda>0$ serves as a canonical splitting manifold for $\mathcal{V}_{p}$, such that under the fibration

$$
2 \pi \mathcal{O}_{-\lambda} \longrightarrow \mathcal{N}(P ; \lambda)
$$

the pullback $\mathrm{q}^{*} \mathcal{V}_{p}$ decomposes smoothly into a direct sum of line bundles over $\mathcal{N}(P ; \lambda)$,

$$
\mathrm{q}^{*} \mathcal{V}_{p} \cong \bigoplus_{j=1}^{r+1} \mathcal{L}_{j}
$$

Hence, the total Chern class of $\mathcal{V}_{p}$ satisfies

$$
\mathrm{q}^{*} c\left(\mathcal{V}_{p}\right)=\prod_{j=1}^{r+1}\left(1+\mathrm{u}_{j}\right), \quad \mathbf{u}_{j}=c_{1}\left(\mathcal{L}_{j}\right) \in H^{2}(\mathcal{N}(P ; \lambda) ; \mathbb{Z})
$$

and $\left\{u_{1}, \ldots, u_{r+1}\right\}$ realize the Chern roots of $\mathcal{V}_{p}$.

To encode the Chern roots in a slightly more invariant fashion, we collect them into a single degree-two class $u$ on $\mathcal{N}(P ; \lambda)$ which is valued in the Cartan subalgebra of $S U(r+1)$,

$$
\mathfrak{u}=i \operatorname{diag}\left(\mathfrak{u}_{1}, \cdots, \mathfrak{u}_{r+1}\right) \in H^{2}(\mathcal{N}(P ; \lambda) ; \mathbb{Z}) \otimes \mathfrak{t} .
$$


The individual Chern roots can then be extracted from $u$ using the basic weights $\left\{\hat{\omega}_{1}, \ldots, \hat{\omega}_{r+1}\right\}$ in $(5.55)$,

$$
\mathbf{u}_{j}=\left\langle\hat{\omega}_{j}, \mathfrak{u}\right\rangle, \quad j=1, \ldots, r+1 .
$$

With the conventions in (5.59), each $\mathfrak{u}_{j}$ restricts fiberwise on $\mathcal{O}_{\lambda}$ to the invariant two-form

$$
\left.\mathrm{u}_{j}\right|_{\mathcal{O}_{\lambda}}=-\frac{\nu_{\hat{\omega}_{j}}}{2 \pi}, \quad j=1, \ldots, r+1
$$

Under the Chern-Weil homomorphism, the degree-two class $u$ in (7.200) plays exactly the role of the variable $\phi \in \mathfrak{t}$ appearing in (7.195). As a result, the characteristic class $\operatorname{ch}_{R}\left(\mathcal{V}_{p}\right)$ is given explicitly in terms of $u$ by

$$
\operatorname{ch}_{R}\left(\mathcal{V}_{p}\right)=\operatorname{ch}_{R}\left(\mathrm{e}^{\mathfrak{u}}\right)=\frac{\mathrm{A}_{\alpha+\rho}(\mathrm{u})}{\mathrm{A}_{\rho}(\mathrm{u})} .
$$

Just as in (7.142), $\mathrm{A}_{\alpha}(\mathrm{u})$ is defined for each weight $\alpha$ via an alternating sum over the Weyl group $\mathfrak{W}$,

$$
\mathrm{A}_{\alpha}(\mathrm{u})=\sum_{w \in \mathfrak{W}}(-1)^{w} \mathrm{e}^{\langle w \cdot \alpha, \mathrm{u}\rangle},
$$

and in passing to the latter expression for $\operatorname{ch}_{R}\left(\mathcal{V}_{p}\right)$ in (7.203), we apply the Weyl character formula. Finally, we abuse notation slightly in (7.203). Most literally, $\operatorname{ch}_{R}\left(\mathcal{V}_{p}\right)$ is a class downstairs on $\mathcal{N}(P)$, whereas $\operatorname{ch}_{R}\left(\mathrm{e}^{\mathrm{u}}\right)$ is a class upstairs on the splitting manifold $\mathcal{N}(P ; \lambda)$. So more correctly, we should write $\mathrm{q}^{*} \operatorname{ch}_{R}\left(\mathcal{V}_{p}\right)=\operatorname{ch}_{R}\left(\mathrm{e}^{\mathrm{u}}\right)$, recalling from (7.199) that any symmetric function of the Chern roots $\left\{u_{1}, \ldots, u_{r+1}\right\}$ is the pullback from a corresponding characteristic class on $\mathcal{N}(P)$. We will henceforth avoid such pedantries, and we will not distinguish between characteristic classes of $\mathcal{V}_{p}$ on $\mathcal{N}(P)$ and their pullbacks written in terms of $\boldsymbol{u}$ on $\mathcal{N}(P ; \lambda)$.

The characteristic class $\operatorname{ch}_{R}\left(\mathcal{V}_{p}\right)$ admits yet another description as the Chern character of a universal bundle $\mathcal{V}_{p}^{R}$ associated to the representation $R$. Here $\mathcal{V}_{p}^{R}$ is the restriction to $\{p\} \times \mathcal{N}(P)$ of a universal bundle $\mathcal{V}^{R}$ with fiber $R$ over $\Sigma \times \mathcal{N}(P)$,

$$
R \longrightarrow V^{\stackrel{V^{R}}{\perp} \mathcal{N}(P)} .
$$


If $R$ is the fundamental, $(r+1)$-dimensional representation of $S U(r+1)$, then $\mathcal{V}^{R}$ is the standard universal bundle $\mathcal{V}$ appearing in (7.193). Otherwise, at least for the familiar example $G=S U(r+1)$, the associated bundle $V^{R}$ can be constructed algebraically from appropriate symmetric and antisymmetric tensor powers of $\mathcal{V}$.

By definition, if $E$ is a complex vector bundle with Chern roots $e_{j}$ for $j=1, \ldots, \mathrm{rk} E$, the Chern character $\operatorname{ch}(E)$ is given by the sum of exponentials

$$
\operatorname{ch}(E)=\sum_{j=1}^{\mathrm{rk} E} \exp \left(e_{j}\right), \quad \exp \left(e_{j}\right)=1+e_{j}+\frac{1}{2} e_{j}^{2}+\cdots
$$

As throughout, the exponential in (7.206) is to be understood by the indicated Taylor series, which eventually terminates at some finite order due to the nilpotency of the $e_{j}$. On the other hand, the Chern roots of the associated bundle $\mathcal{V}_{p}^{R}$ are by construction the eigenvalues of $u$ acting as an element of $\mathfrak{t}$ on the representation $R$. So tautologically, the Chern character of $\mathcal{V}_{p}^{R}$ is given by

$$
\begin{aligned}
\operatorname{ch}\left(\mathcal{V}_{p}^{R}\right) & =\operatorname{Tr}_{R}\left(\mathrm{e}^{\mathrm{u}}\right) \equiv \operatorname{ch}_{R}\left(\mathrm{e}^{\mathrm{u}}\right), \\
& =\operatorname{ch}_{R}\left(\mathcal{V}_{p}\right) .
\end{aligned}
$$

From the $K$-theory perspective adopted in [101], the alternative description of the Seifert loop class as the Chern character $\operatorname{ch}\left(\mathcal{V}_{p}^{R}\right)$ is possibly more natural.

\subsubsection{Warm-up: index theory and the Seifert loop operator on $S^{1} \times \Sigma$}

In order to analyze the Seifert loop path integral via non-abelian localization, we must assume that the degree $n$ of the bundle $S^{1} \stackrel{n}{\longrightarrow} M \stackrel{\pi}{\longrightarrow} \Sigma$ is nonzero. Otherwise, if $n=0$ and $M=S^{1} \times \Sigma$, the construction of the invariant contact form $\kappa$ in Section 3.2 fails - see especially (3.14) - and our analysis of Chern-Simons theory via localization does not apply.

Regardless, localization or no, Chern-Simons gauge theory simplifies dramatically on a three-manifold which is a product $S^{1} \times \Sigma$. In this situation, as we quickly review below, the Seifert loop path integral $Z(\epsilon ; C, R)$ merely computes the dimension of a certain Hilbert space, which is constructed geometrically as the space of holomorphic sections of a prequantum line bundle over $\mathcal{N}(P ; \lambda)$. Since our identification (7.196) of the Seifert loop class as the 
"ATMP-17-1-A1-BEA" — 2013/5/15 — 19:32 — page 188 — \#188

character $\operatorname{ch}_{R}\left(\mathcal{V}_{p}\right)$ is independent of the degree $n$, one might wonder whether that identification can be understood more easily in the special case $n=0$.

Somewhat remarkably, this question has already been answered by Jeffrey in work [65] that substantially predates the current paper. (See also [58] for a related follow-up.) Indeed, many of the most important ideas required to analyze the Seifert loop path integral for general degree $n \geq 1$ already appear for the somewhat degenerate case $n=0$. Therefore, as a very useful prelude to our localization computations, let us first discuss the Seifert loop path integral on $M=S^{1} \times \Sigma$, closely following the relevant portions of [65].

By way of comparison and contrast, we consider simultaneously both the partition function $Z(\epsilon)$ and the Seifert loop path integral $Z(\epsilon ; C, R)$ for Chern-Simons theory at level $k$ on $M=S^{1} \times \Sigma$. The curve $C$ associated to the Seifert loop operator is then given by $C=S^{1} \times\{p\}$, where $p$ is a point on $\Sigma$.

When $M=S^{1} \times \Sigma$ factorizes, Chern-Simons theory on $M$ can be immediately analyzed via canonical quantization in the Hamiltonian formalism, just as we applied to the Wilson loop operator itself in Section 4.1. In the Hamiltonian formalism, we interpret the $S^{1}$ factor of $M$ as a periodic, Euclidean "time". According to the standard axioms of quantum field theory, the Chern-Simons partition function $Z(\epsilon)$ on $M$ is then generally given by a quantum mechanical trace of the form

$$
Z(\epsilon)=\operatorname{Tr}_{\mathcal{H}(k)} P \exp \left(-\mathrm{i} \oint_{S^{1}} \mathbf{H}\right), \quad M=S^{1} \times \Sigma .
$$

Here $\mathcal{H}(k)$ is the finite-dimensional Hilbert space obtained by quantizing Chern-Simons theory at level $k$ on $\Sigma$, and $\mathbf{H}$ is the Hamiltonian which acts on $\mathcal{H}(k)$ to generate infinitesimal translations around $S^{1}$. Because the Chern-Simons action is purely topological, the Hamiltonian vanishes,

$$
\mathbf{H}=0 .
$$

Hence $Z(\epsilon)$ reduces to the trace of the identity operator on $\mathcal{H}(k)$,

$$
Z(\epsilon)=\operatorname{Tr}_{\mathcal{H}(k)} \mathbf{1}=\operatorname{dim}_{\mathbb{C}} \mathcal{H}(k),
$$

and the Chern-Simons path integral on $S^{1} \times \Sigma$ simply computes the dimension of the Hilbert space $\mathcal{H}(k)$.

For the Seifert loop path integral, the axiomatic reasoning, which leads to (7.210) remains valid. Again, $Z(\epsilon ; C, R)$ is given by the dimension of a 
"ATMP-17-1-A1-BEA" — 2013/5/15 — 19:32 — page 189 — \#189

LOCALIZATION FOR WILSON LOOPS IN CHERN-SIMONS

Hilbert space,

$$
Z(\epsilon ; C, R)=\operatorname{Tr}_{\mathcal{H}(k ; \alpha)} \mathbf{1}=\operatorname{dim}_{\mathbb{C}} \mathcal{H}(k ; \alpha)
$$

where $\mathcal{H}(k ; \alpha)$ is now obtained by quantizing Chern-Simons theory at level $k$ in the presence of the Seifert loop operator $W_{R}(C)$ which punctures $\Sigma$ at the point $p$.

As nicely reviewed in [10], each of the Hilbert spaces $\mathcal{H}(k)$ and $\mathcal{H}(k ; \alpha)$ can be constructed geometrically as the space of holomorphic sections of a certain prequantum line bundle over the appropriate classical phase space of Chern-Simons theory. In the case of $\mathcal{H}(k)$, the relevant phase space is just the moduli space $\mathcal{N}(P)$ of flat connections on $\Sigma$, so that ${ }^{40}$

$$
\mathcal{H}(k) \cong H_{\bar{\partial}}^{0}\left(\mathcal{N}(P), \mathcal{L}_{0}^{k}\right)
$$

Here the prequantum line bundle $\mathcal{L}_{0}^{k}$ is the $k$ th power of the basic line bundle $\mathcal{L}_{0}$, which generates the Picard group of $\mathcal{N}(P)$ and whose first Chern class is represented on $\mathcal{N}(P)$ by

$$
c_{1}\left(\mathcal{L}_{0}\right)=\Omega_{0}=\frac{1}{4 \pi^{2}} \Omega .
$$

We recall that $\Omega$ is the symplectic form appearing in (2.2). With our normalization conventions, the periods of $\Omega$ are given by integral multiples of $4 \pi^{2}$, so the normalization factor in (7.213) ensures that $\Omega_{0}$ is an integral generator of $H^{2}(\mathcal{N}(P) ; \mathbb{Z}) \cong \mathbb{Z}$.

The geometric description of $\mathcal{H}(k ; \alpha)$ may be only marginally less familiar. As we have seen in Section 5.1, classical solutions of Chern-Simons theory in the presence of the Seifert loop operator $W_{R}(C)$ are described on $\Sigma$ by the extended moduli space $\mathcal{N}(P ; \lambda)$, where the parameter $\lambda$ is fixed as in (7.17) by the ratio

$$
\lambda=\frac{\alpha}{k}
$$

\footnotetext{
${ }^{40}$ The following caveat is in order. If the Chern-Simons gauge group $G$ is simplyconnected, then the $G$-bundle $P$ on $\Sigma$ is topologically trivial, and $\mathcal{N}(P)$ has singularities at points corresponding to reducible flat connections on $\Sigma$. At present, we proceed somewhat formally and ignore singularities in $\mathcal{N}(P)$. Lest the reader worry, when we return to this discussion in the context of localization, $P$ will be non-trivial and $\mathcal{N}(P)$ will be smooth.
} 
Hence, the Hilbert space $\mathcal{H}(k ; \alpha)$ which describes the Seifert loop operator is constructed by quantizing a new phase space, namely $\mathcal{N}(P ; \lambda)$,

$$
\mathcal{H}(k ; \alpha) \cong H_{\bar{\partial}}^{0}\left(\mathcal{N}(P ; \lambda), \mathcal{L}_{\alpha}^{(k)}\right)
$$

where $\mathcal{L}_{\alpha}^{(k)}$ is the requisite prequantum line bundle over $\mathcal{N}(P ; \lambda)$.

Unlike the prequantum line bundle $\mathcal{L}_{0}^{k}$ which defines the Hilbert space $\mathcal{H}(k)$, the line bundle $\mathcal{L}_{\alpha}^{(k)}$ is not generally the $k$ th power of any more basic line bundle on $\mathcal{N}(P ; \lambda)$. However, again up to factors of $2 \pi$, the first Chern class of $\mathcal{L}_{\alpha}^{(k)}$ is still represented on $\mathcal{N}(P ; \lambda)$ by the $k$ th multiple of the symplectic form $\Omega_{\lambda}$ in (5.32),

$$
c_{1}\left(\mathcal{L}_{\alpha}^{(k)}\right)=\frac{k}{4 \pi^{2}} \Omega_{\lambda}=k \mathrm{q}^{*} \Omega_{0}+\langle\alpha, \mathrm{u}\rangle .
$$

Here, along with the elementary identification in (7.214), we use the description (5.65) of $\Omega_{\lambda}$ in terms of the Chern roots $u$ of $\mathcal{V}_{p}$. This description of $\Omega_{\lambda}$ is valid so long as the symplectic fibration $\mathrm{q}: \mathcal{N}(P ; \lambda) \rightarrow \mathcal{N}(P)$ in (7.197) is itself valid, for which we require the strict integrability bound $(\vartheta, \alpha)<k$ on the weight $\alpha$.

According to Theorem 9.12 in $[12], \mathcal{N}(P)$ is simply-connected. Via the symplectic fibration in (7.197), $\mathcal{N}(P ; \lambda)$ is simply-connected as well. Hence, our expression for the Chern class of $\mathcal{L}_{\alpha}^{(k)}$ in (7.216) suffices to determine the line bundle itself. To be precise, since $\Omega_{0}$ represents the Chern class of the generating line bundle $\mathcal{L}_{0}$ on $\mathcal{N}(P)$, and since each Chern root $u_{j}$ represents the Chern class of the splitting line bundle $\mathcal{L}_{j}$ in (7.198), the sum in (7.216) implies that $\mathcal{L}_{\alpha}^{(k)}$ is given by the tensor product

$$
\mathcal{L}_{\alpha}^{(k)} \cong \mathrm{q}^{*} \mathcal{L}_{0}^{k} \otimes \bigotimes_{j=1}^{r+1} \mathcal{L}_{j}^{\alpha_{j}},
$$

where $\alpha_{j}$ for $j=1, \ldots, r+1$ are the components of $\alpha$ in (7.191). The quantization of $\alpha$ as a weight of $S U(r+1)$ is necessary to ensure that the tensor product of line bundles $\mathcal{L}_{j}^{\alpha_{j}}$ in (7.217) is well-defined. To check that the tensor product is sensible, we observe that the product $\otimes_{j=1}^{r+1} \mathcal{L}_{j}^{\alpha_{j}}$ can be rewritten as $\otimes_{j=1}^{r} \mathcal{L}_{j}^{\alpha_{j}-\alpha_{r+1}}$, where we eliminate the redundant bundle $\mathcal{L}_{r+1}$ via the relation $\mathcal{L}_{r+1} \cong \otimes_{j=1}^{r} \mathcal{L}_{j}^{-1}$, which follows from the elementary identity $\hat{\omega}_{1}+\cdots+\hat{\omega}_{r+1}=0$. In our conventions, with $\alpha>0$ a positive weight of $S U(r+1)$, the differences $\alpha_{j}-\alpha_{r+1} \in \mathbb{Z}_{>0}$ for $j=1, \ldots, r$ are then strictly 
positive integers, and no fractional powers of line bundles appear in the tensor product $\otimes_{j=1}^{r} \mathcal{L}_{j}^{\alpha_{j}-\alpha_{r+1}}$.

To summarize, for Chern-Simons theory on $M=S^{1} \times \Sigma$, both $Z(\epsilon)$ and $Z(\epsilon ; C, R)$ count holomorphic sections of appropriate prequantum line bundles on $\mathcal{N}(P)$ and $\mathcal{N}(P ; \lambda)$,

$$
\begin{aligned}
Z(\epsilon) & =\operatorname{dim}_{\mathbb{C}} H \frac{0}{\partial}\left(\mathcal{N}(P), \mathcal{L}_{0}^{k}\right) \\
Z(\epsilon ; C, R) & =\operatorname{dim}_{\mathbb{C}} H_{\bar{\partial}}^{0}\left(\mathcal{N}(P ; \lambda), \mathrm{q}^{*} \mathcal{L}_{0}^{k} \otimes \bigotimes_{j=1}^{r+1} \mathcal{L}_{j}^{\alpha_{j}}\right)
\end{aligned}
$$

Crucially, for both prequantum line bundles appearing in (7.218), the higher Dolbeault cohomology vanishes. As noted for instance in Section 11 of [64], the vanishing of $H_{\frac{q}{\partial}}\left(\mathcal{N}(P), \mathcal{L}_{0}^{k}\right)$ for $q>0$ follows from the Kodaira vanishing theorem, in combination with the identification of the canonical bundle of $\mathcal{N}(P)$ as $\mathcal{L}_{0}^{-2}$. The vanishing of $H_{\frac{q}{\partial}}^{q}\left(\mathcal{N}(P ; \lambda), \mathcal{L}_{\alpha}^{(k)}\right)$ for $q>0$ is apparently a bit more subtle, but it has been proven by Teleman in (9.6) of [102]. As a result, both $Z(\epsilon)$ and $Z(\epsilon ; C, R)$ are alternatively given by the holomorphic Euler characters

$$
\begin{aligned}
Z(\epsilon) & =\chi\left(\mathcal{N}(P), \mathcal{L}_{0}^{k}\right) \\
Z(\epsilon ; C, R) & =\chi\left(\mathcal{N}(P ; \lambda), \mathrm{q}^{*} \mathcal{L}_{0}^{k} \otimes \bigotimes_{j=1}^{r+1} \mathcal{L}_{j}^{\alpha_{j}}\right)
\end{aligned}
$$

Let us recall one basic fact about the holomorphic Euler character. In general, if $E$ is a holomorphic vector bundle over a compact complex manifold $X$, the holomorphic Euler character $\chi(X, E)$ is defined by the alternating sum

$$
\chi(X, E)=\sum_{q}(-1)^{q} \operatorname{dim}_{\mathbb{C}} H_{\frac{q}{\partial}}^{q}(X, E) .
$$

The Atiyah-Singer index theorem, in its Hirzebruch-Riemann-Roch form, then provides an entirely cohomological description for the Euler character,

$$
\chi(X, E)=\int_{X} \operatorname{ch}(E) \cdot \operatorname{Td}(X) .
$$

We have already encountered the Chern character $\operatorname{ch}(E)$ in (7.206). Otherwise, $\operatorname{Td}(X)$ is the Todd class of $X$. Explicitly, if $x_{j}$ for $j=1, \ldots, \operatorname{dim}_{\mathbb{C}} X$ 
are the Chern roots of the complex tangent bundle of $X$, such that

$$
c(X)=\prod_{j=1}^{\operatorname{dim}_{\mathbb{C}} X}\left(1+x_{j}\right),
$$

then $\operatorname{Td}(X)$ is given by the product

$$
\operatorname{Td}(X)=\prod_{j=1}^{\operatorname{dim}_{\mathbb{C}} X} \frac{x_{j}}{1-\exp \left(-x_{j}\right)} .
$$

Applying the Hirzebruch-Riemann-Roch formula in (7.221) to (7.219), we thereby express $Z(\epsilon)$ and $Z(\epsilon ; C, R)$ as integrals over the respective moduli spaces $\mathcal{N}(P)$ and $\mathcal{N}(P ; \lambda)$,

$$
\begin{aligned}
Z(\epsilon) & =\int_{\mathcal{N}(P)} \exp \left[k \Omega_{0}\right] \cdot \operatorname{Td}(\mathcal{N}(P)) \\
Z(\epsilon ; C, R) & =\int_{\mathcal{N}(P ; \lambda)} \exp \left[k \mathrm{q}^{*} \Omega_{0}+\langle\alpha, \mathfrak{u}\rangle\right] \cdot \operatorname{Td}(\mathcal{N}(P ; \lambda)) .
\end{aligned}
$$

In obtaining (7.224), we trivially note that $\operatorname{ch}(E)=\exp \left[c_{1}(E)\right]$ when $E$ is a line bundle, and we substitute for the respective Chern classes using (7.213) and (7.216).

Although (7.224) provides the sought-after cohomological description for $Z(\epsilon ; C, R)$, we still need to tease out a description of the Seifert loop class itself,

$$
\left.W_{R}(C)\right|_{\mathcal{N}(P)} \in H^{*}(\mathcal{N}(P))
$$

Intuitively, the Seifert loop class $\left.W_{R}(C)\right|_{\mathcal{N}(P)}$ should play the same role in the presentation of $Z(\epsilon ; C, R)$ as an integral over the finite-dimensional moduli space $\mathcal{N}(P)$ that the Seifert loop operator $W_{R}(C)$ itself plays in the full Seifert loop path integral over the infinite-dimensional affine space $\mathcal{A}$ of connections,

$$
\begin{aligned}
Z(\epsilon ; C, R)= & \frac{1}{\operatorname{Vol}(\mathcal{G})}\left(\frac{1}{2 \pi \epsilon}\right)^{\Delta_{\mathcal{G}}} \int_{\mathcal{A}} \mathcal{D} A W_{R}(C) \\
& \times \exp \left[\frac{\mathrm{i}}{2 \epsilon} \int_{M} \operatorname{Tr}\left(A \wedge d A+\frac{2}{3} A \wedge A \wedge A\right)\right] \\
\epsilon= & \frac{2 \pi}{k}, \quad \Delta_{\mathcal{G}}=\operatorname{dim} \mathcal{G} .
\end{aligned}
$$


Here the Seifert loop path integral (7.226) is obtained by multiplying the exponential of the Chern-Simons action by the operator $W_{R}(C)$, interpreted as a classical functional of the connection $A$.

Similarly, the Seifert loop class will be characterized as the element of $H^{*}(\mathcal{N}(P))$ by which we multiply the index theory integrand of $Z(\epsilon)$ in (7.224) to obtain a corresponding cohomological formula for $Z(\epsilon ; C, R)$,

$$
Z(\epsilon ; C, R)=\left.\int_{\mathcal{N}(P)} W_{R}(C)\right|_{\mathcal{N}(P)} \cdot \exp \left[k \Omega_{0}\right] \cdot \operatorname{Td}(\mathcal{N}(P)) .
$$

Crucially, $Z(\epsilon ; C, R)$ is expressed in (7.227) as an integral over the basic moduli space $\mathcal{N}(P)$, as opposed to the extended moduli space $\mathcal{N}(P ; \lambda)$. To compute the Seifert loop class, we must therefore push the index density on $\mathcal{N}(P ; \lambda)$ in $(7.224)$ down to $\mathcal{N}(P)$ by integrating over the fiber $2 \pi \mathcal{O}_{-\lambda}$ of the $\operatorname{map} \mathrm{q}: \mathcal{N}(P ; \lambda) \rightarrow \mathcal{N}(P)$,

$$
Z(\epsilon ; C, R)=\int_{\mathcal{N}(P)} \mathbf{q}_{*}\left[\exp \left(k \mathrm{q}^{*} \Omega_{0}+\langle\alpha, \mathrm{u}\rangle\right) \cdot \operatorname{Td}(\mathcal{N}(P ; \lambda))\right] .
$$

After simplifying the expression in (7.228) and comparing the result to (7.227), we ultimately determine $\left.W_{R}(C)\right|_{\mathcal{N}(P)}$. As will be clear, this calculation nicely parallels the more elementary manipulations leading to (7.151) in Section 7.2.

To begin to simplify (7.228), let us express the Todd class of $\mathcal{N}(P ; \lambda)$ in a more revealing form. In general, for any holomorphic fibration of smooth complex manifolds

$$
\begin{gathered}
F \longrightarrow \\
\underset{B}{\downarrow} \pi \\
{ }_{B} \pi
\end{gathered}
$$

the Todd class of $X$ factorizes as

$$
\operatorname{Td}(X)=\operatorname{Td}(F) \cdot \pi^{*} \operatorname{Td}(B) .
$$

As standard, the factorization in (7.230) follows from the corresponding exact sequence of bundles

$$
0 \longrightarrow T F \longrightarrow T X \stackrel{\pi_{*}}{\longrightarrow} T B \longrightarrow 0,
$$

along with the manifestly multiplicative definition (7.223) of $\operatorname{Td}(X)$ as a product over the individual Chern roots of the holomorphic tangent bundle $T X$. For the fibration $\mathrm{q}: \mathcal{N}(P ; \lambda) \rightarrow \mathcal{N}(P)$ with the natural complex 
"ATMP-17-1-A1-BEA" — 2013/5/15 — 19:32 — page 194 — \#194

structures on all spaces involved, we obtain

$$
\operatorname{Td}(\mathcal{N}(P ; \lambda))=\operatorname{Td}\left(\mathcal{O}_{-\lambda}\right) \cdot \mathrm{q}^{*} \operatorname{Td}(\mathcal{N}(P)) .
$$

Let us make a comment, not inessential, about the appearance of $\mathcal{O}_{-\lambda}$ as opposed to $\mathcal{O}_{\lambda}$ in (7.232). According to (7.197), the fiber of the map $q$ is symplectically the orbit $2 \pi \mathcal{O}_{-\lambda}$. For the purpose of computing the fiberwise Todd class, the overall normalization of the orbit by $2 \pi$ is irrelevant, so we have dropped that normalization in (7.232). However, if we are going to be careful about signs and orientations, the distinction between $\lambda$ and $-\lambda$ in (7.232) is important.

According to (4.23), when $\lambda>0$ is positive, the complex structure on $\mathcal{O}_{\lambda}$ compatible with the symplectic form $\nu_{\lambda}$ is such that the holomorphic tangent space to $\mathcal{O}_{\lambda}$ at the identity is given by $\mathfrak{g}^{(1,0)}=\mathfrak{g}_{+}$, the positive rootspace of $\mathfrak{g}$. However, because the sign of the symplectic form on $\mathcal{O}_{-\lambda}$ is reversed relative to $\mathcal{O}_{\lambda}$, the compatible complex structure on $\mathcal{O}_{-\lambda}$ is also reversed. That is, the holomorphic tangent space to $\mathcal{O}_{-\lambda}$ at the identity is given by the negative rootspace $\mathfrak{g}_{-}$(for a fixed splitting $\mathfrak{g}_{\mathbb{C}} \ominus \mathfrak{t}_{\mathbb{C}}=\mathfrak{g}_{+} \oplus \mathfrak{g}_{-}$). So even though $\mathcal{O}_{\lambda}$ and $\mathcal{O}_{-\lambda}$ agree at the level of topology, they carry opposite complex structures. Eventually, this distinction will be manifested through various signs in $\operatorname{Td}\left(\mathcal{O}_{-\lambda}\right)$ relative to $\operatorname{Td}\left(\mathcal{O}_{\lambda}\right)$, associated to the exchange $\mathfrak{g}_{+} \leftrightarrow \mathfrak{g}_{-}$.

Via (7.232), the index density on $\mathcal{N}(P ; \lambda)$ itself factorizes, implying

$$
Z(\epsilon ; C, R)=\int_{\mathcal{N}(P ; \lambda)} \exp [\langle\alpha, \mathfrak{u}\rangle] \cdot \operatorname{Td}\left(\mathcal{O}_{-\lambda}\right) \cdot \mathrm{q}^{*}\left(\exp \left[k \Omega_{0}\right] \operatorname{Td}(\mathcal{N}(P))\right)
$$

In particular, comparing to (7.224), we see the pullback of the index density on $\mathcal{N}(P)$ appears as the final factor in the integrand of (7.233). Trivially, terms which pull back from the base $\mathcal{N}(P)$ do not contribute to the integral over the fiber of $\mathcal{N}(P ; \lambda)$. Consequently, the Seifert loop class in $(7.227)$ is given by the pushdown of the first two factors in the integrand of (7.233),

$$
\left.W_{R}(C)\right|_{\mathcal{N}(P)}=\mathrm{q}_{*} S_{\alpha}(\mathrm{u}) \in H^{*}(\mathcal{N}(P)),
$$

where we set

$$
S_{\alpha}(\mathfrak{u})=\exp [\langle\alpha, u\rangle] \cdot \operatorname{Td}\left(\mathcal{O}_{-\lambda}\right) .
$$

Evaluating $\mathrm{q}_{*} S_{\alpha}(\mathrm{u})$ will be easy as soon as we determine the Todd class of the orbit $\mathcal{O}_{-\lambda}$. We accomplish this task directly, by computing the Chern roots of $\mathcal{O}_{-\lambda}$ and then applying the definition in (7.223). 
Let us first make a simple observation about signs. If $x_{j}$ for $j=1, \ldots$, $\operatorname{dim}_{\mathbb{C}} \mathcal{O}_{\lambda}$ are the Chern roots of the coadjoint orbit $\mathcal{O}_{\lambda}$ with the standard complex structure in (4.23),

$$
c\left(\mathcal{O}_{\lambda}\right)=\prod_{j=1}^{\operatorname{dim}_{\mathbb{C}} \mathcal{O}_{\lambda}}\left(1+x_{j}\right),
$$

then the Chern roots of the conjugate orbit $\mathcal{O}_{-\lambda}$, which carries the opposite complex structure, are given by $-x_{j}$ for $j=1, \ldots, \operatorname{dim}_{\mathbb{C}} \mathcal{O}_{\lambda}$,

$$
c\left(\mathcal{O}_{-\lambda}\right)=\prod_{j=1}^{\operatorname{dim}_{\mathbb{C}} \mathcal{O}_{\lambda}}\left(1-x_{j}\right)
$$

Because of this relation, we have decided to phrase our initial discussion in terms of the more familiar Chern roots $x_{j}$ of $\mathcal{O}_{\lambda}$. We then reverse signs to compute the Chern roots of $\mathcal{O}_{-\lambda}$.

The computation of the Chern roots of the complete flag manifold $\mathcal{O}_{\lambda} \cong$ $G / T$ is a classic result, so we will be brief. See Section 14.2 of [59] for a textbook discussion. Because $\mathcal{O}_{\lambda}$ is a homogeneous space for $G$, the Chern roots of $\mathcal{O}_{\lambda}$ are determined by any smooth splitting of the holomorphic tangent space $\mathfrak{g}^{(1,0)}=\mathfrak{g}_{+}$at the identity. The obvious splitting at hand is the eigenspace decomposition of $\mathfrak{g}_{+}$under the action of the maximal torus $T$,

$$
\mathfrak{g}_{+} \cong \bigoplus_{\beta>0} \mathfrak{e}_{\beta}, \quad \beta \in \mathfrak{R}
$$

where $\mathfrak{e}_{\beta}$ is the one-dimensional rootspace associated to the root $\beta$ of $G$. Each rootspace $\mathfrak{e}_{\beta}$ then varies globally over $\mathcal{O}_{\lambda} \cong G / T$ as the fiber of the corresponding homogeneous line bundle $\mathfrak{L}(\beta)$, as in (4.32). So via the splitting in (7.238), the Chern classes of the line bundles $\mathfrak{L}(\beta)$ for all $\beta>0$ realize the Chern roots of $\mathcal{O}_{\lambda}$,

$$
c\left(\mathcal{O}_{\lambda}\right)=\prod_{\beta>0}\left[1+c_{1}(\mathfrak{L}(\beta))\right], \quad c_{1}(\mathfrak{L}(\beta))=\frac{\nu_{\beta}}{2 \pi} .
$$

Equivalently, after reversing signs to account for the reversal of complex structure on $\mathcal{O}_{-\lambda}$,

$$
c\left(\mathcal{O}_{-\lambda}\right)=\prod_{\beta>0}\left[1-c_{1}(\mathfrak{L}(\beta))\right] .
$$


As a check, if $G=S U(2)$, then $\mathcal{O}_{\lambda}=\mathbb{C} \mathbb{P}^{1}$. Previously in the example in Section 4.1, we identified the line bundle of degree $m$ on $\mathbb{C P}^{1}$ with the homogeneous line bundle $\mathfrak{L}(m \hat{\omega})$, where $\hat{\omega}>0$ is the highest weight of the fundamental, two-dimensional representation of $S U(2)$. Observing that $\beta=2 \hat{\omega}$ in (7.239), we obtain the standard result that the first Chern class of $\mathbb{C P}^{1}$ has degree two.

To compute $\operatorname{Td}\left(\mathcal{O}_{-\lambda}\right)$ as a class on $\mathcal{N}(P ; \lambda)$, we now express the Chern class of each line bundle $\mathfrak{L}(\beta)$ in terms of the fiberwise components of the fundamental Chern roots $u$ of $\mathcal{V}_{p}$. According to (4.35) and (5.59),

$$
c_{1}(\mathfrak{L}(\beta))=\frac{\nu_{\beta}}{2 \pi}=-\left.\langle\beta, \mathfrak{u}\rangle\right|_{\mathcal{O}_{\lambda}} .
$$

Substituting into (7.240), we then obtain the simple result

$$
c\left(\mathcal{O}_{-\lambda}\right)=\prod_{\beta>0}(1+\langle\beta, \mathfrak{u}\rangle)
$$

where the signs in (7.240) and (7.241) nicely cancel.

Consequently,

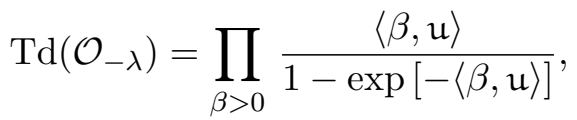

$$
\begin{aligned}
& =\prod_{\beta>0} \frac{\mathrm{e}^{\langle\beta, \mathrm{u}\rangle / 2}}{2 \sinh (\langle\beta, \mathfrak{u}\rangle / 2)} \cdot\langle\beta, \mathfrak{u}\rangle, \\
& =\frac{\mathrm{e}^{\langle\rho, \mathrm{u}\rangle}}{\mathrm{A}_{\rho}(\mathrm{u})} \cdot \prod_{\beta>0}\langle\beta, \mathrm{u}\rangle, \quad \rho=\frac{1}{2} \sum_{\beta>0} \beta .
\end{aligned}
$$

The second equality in (7.243) is an elementary algebraic identity, and in passing to the last line of (7.243), we apply the Weyl denominator formula (7.145) for $\mathrm{A}_{\rho}$.

With this description of $\operatorname{Td}\left(\mathcal{O}_{-\lambda}\right)$, the class $S_{\alpha}(\mathfrak{u})$ in (7.235) becomes

$$
S_{\alpha}(\mathfrak{u})=\frac{\mathrm{e}^{\langle\alpha+\rho, \mathfrak{u}\rangle}}{\mathrm{A}_{\rho}(\mathfrak{u})} \cdot \prod_{\beta>0}\langle\beta, \mathfrak{u}\rangle .
$$

To finish our computation of the Seifert loop class $\left.W_{R}(C)\right|_{\mathcal{N}(P)}$, we are left to push $S_{\alpha}(\mathrm{u})$ down to $\mathcal{N}(P)$ under the map q : $\mathcal{N}(P ; \lambda) \rightarrow \mathcal{N}(P)$. 
To orient the reader, let us quickly recall how pushdown works. See Section 6 of [25] for a more thorough discussion in the special case of vector bundles. As in (7.229), we suppose that we have a smooth fibration of compact manifolds $F \rightarrow X \stackrel{\pi}{\rightarrow} B .^{41}$ Locally, with respect to a given trivialization over a patch of $B$, any differential form $\psi$ on $X$ can be written as a finite sum of terms

$$
\psi=\sum_{i} \pi^{*} \xi_{(i)} \wedge \eta_{(i)}
$$

where each $\xi_{(i)}$ is a differential form on $B$, and each $\eta_{(i)}$ is a vertical differential form on $X$. That is, $\eta_{(i)}$ has components only along the fiber $F$, or equivalently, the contraction of $\eta_{(i)}$ with any horizontal tangent vector along $B$ vanishes.

As a map from differential forms on $X$ to differential forms on $B$, the pushdown $\pi_{*}$ is given by the naive operation of integrating each vertical component $\eta_{(i)}$ of $\psi$ fiberwise over $F$,

$$
\pi_{*} \psi=\sum_{i} \xi_{(i)} \cdot \int_{F} \eta_{(i)}
$$

By definition, if $\eta_{(i)}$ has no component of top degree on $F$, the integral over $F$ is set to zero. As in Section 6 of [25], one can check that the operation in (7.246) does not depend upon the particular choice of local trivialization used to obtain the fiberwise decomposition of $\psi$ in (7.245), so long as the trivialization respects the relative orientations of $X$ and $F$. Hence $\pi_{*}$ is well-defined globally on $X$. Moreover, $\pi_{*}$ commutes with the exterior derivative $d$ and thus induces a map on de Rham cohomology $\pi_{*}: H^{*}(X) \rightarrow H^{*-\operatorname{dim} F}(B)$, where we observe that the integration in (7.246) reduces the degree of a form on $X$ by the dimension of $F$.

We now return to the specific problem of computing the pushdown $\mathfrak{q}_{*} S_{\alpha}(\mathfrak{u})$, for which the essential step is simply to write $S_{\alpha}(u)$ in the form (7.245). Let us therefore consider the Weyl action by permutations on the entries of $\mathfrak{u}=i \operatorname{diag}\left(\mathfrak{u}_{1}, \ldots, \mathfrak{u}_{r+1}\right)$. Because $\left(\mathfrak{u}_{1}, \ldots, \mathfrak{u}_{r+1}\right)$ are the Chern roots of the bundle $\mathcal{V}_{p}$ over $\mathcal{N}(P)$, any symmetric function of $\left(u_{1}, \ldots, u_{r+1}\right)$ is tautologically the pullback from $\mathcal{N}(P)$ of a corresponding function of the Chern classes of $\mathcal{V}_{p}$.

On the other hand, as we have already seen in (7.242), the fiberwise components of the classes $\langle\beta, \mathfrak{u}\rangle$ for all $\beta>0$ represent the Chern roots of

\footnotetext{
${ }^{41}$ We phrase the discussion here in the context of differential topology, and we do not necessarily assume that $F, X$, and $B$ are complex manifolds.
} 
$\mathcal{O}_{-\lambda}$. Let us focus attention on the product over $\langle\beta, \mathfrak{u}\rangle$ occurring as the final factor in (7.244), for which we introduce the shorthand

$$
\eta=\prod_{\beta>0}\langle\beta, \mathfrak{u}\rangle
$$

This differential form $\eta$ on $\mathcal{N}(P ; \lambda)$ has two important features.

First, since the Weyl group $\mathfrak{W}$ is generated by reflections in the simple roots, $\eta$ is manifestly alternating under the action of $\mathfrak{W}$.

Second, $\eta$ restricts to a non-vanishing top-form on each fiber of $\mathcal{N}(P ; \lambda)$. For this claim, we note that each Chern root $\mathfrak{u}_{j}$ restricts via (5.59) to an invariant two-form on $\mathcal{O}_{-\lambda}$, so $\eta$ is also invariant. As a result, $\eta$ will be non-vanishing on $\mathcal{O}_{-\lambda}$ so long as the integral of $\eta$ over $\mathcal{O}_{-\lambda}$ is itself nonvanishing. But the product of Chern roots in (7.247) represents the top Chern class of $\mathcal{O}_{-\lambda}$, so the fiberwise integral of $\eta$ computes the topological Euler character $\chi_{\text {top }}$ of $\mathcal{O}_{-\lambda} \cong G / T$,

$$
\begin{aligned}
\int_{\mathcal{O}_{-\lambda}} \eta & =\chi_{\text {top }}(G / T), \\
& =|\mathfrak{W}| .
\end{aligned}
$$

As we review at the conclusion of this discussion, for any compact simple Lie group $G$, the Euler character of $G / T$ is given by the order of the Weyl group $\mathfrak{W}$, which is certainly non-zero. In the case $G=\mathrm{SU}(r+1)$ of present relevance, $\mathfrak{W}$ is the symmetric group acting by permutations on $\left\{\mathfrak{u}_{1}, \ldots, u_{r+1}\right\}$, so $|\mathfrak{W}|=(r+1) !$

As apparent from these observations, in any fiberwise decomposition of $S_{\alpha}(\mathrm{u})$ as in (7.245), each top-degree vertical form appearing in the decomposition must be proportional to $\eta$ and is thus alternating under $\mathfrak{W}$. Otherwise, each horizontal form which pulls back from $\mathcal{N}(P)$ under $\mathrm{q}^{*}$ is automatically invariant under $\mathfrak{W}$. Consequently, in the fiberwise decomposition of $S_{\alpha}(\mathfrak{u})$, only those summands which are themselves alternating under $\mathfrak{W}$ can contribute under the pushdown $\mathrm{q}_{*}$.

To pick out the alternating piece of

$$
S_{\alpha}(\mathfrak{u})=\frac{\mathrm{e}^{\langle\alpha+\rho, \mathrm{u}\rangle}}{\mathrm{A}_{\rho}(\mathrm{u})} \cdot \eta,
$$

we note that both $\eta$ and $\mathrm{A}_{\rho}(\mathrm{u})$ are already alternating. So the alternating piece of $S_{\alpha}(\mathrm{u})$ is obtained by anti-symmetrizing the remaining exponential 
factor exactly as in (7.149),

$$
\begin{aligned}
\mathrm{A}\left[\mathrm{e}^{\langle\alpha+\rho, \mathfrak{u}\rangle}\right] & =\frac{1}{|\mathfrak{W}|} \sum_{w \in \mathfrak{W}}(-1)^{w} \mathrm{e}^{\langle w \cdot(\alpha+\rho), \mathfrak{u}\rangle} \\
& =\frac{1}{|\mathfrak{W}|} \mathrm{A}_{\alpha+\rho}(\mathfrak{u})
\end{aligned}
$$

Thus we write $S_{\alpha}(\mathfrak{u})$ as

$$
\begin{aligned}
S_{\alpha}(\mathfrak{u}) & =\frac{1}{|\mathfrak{W}|} \frac{\mathrm{A}_{\alpha+\rho}(\mathrm{u})}{\mathrm{A}_{\rho}(\mathrm{u})} \cdot \eta+\cdots, \\
& =\frac{1}{|\mathfrak{W}|} \mathrm{q}^{*} \operatorname{ch}_{R}\left(\mathcal{V}_{p}\right) \cdot \eta+\cdots,
\end{aligned}
$$

where the "..." indicate terms which are annihilated by $\mathrm{q}_{*}$, and in the second line of (7.251) we apply the character formula as in (7.203).

Finally, integrating $\eta$ over the fiber of $\mathcal{N}(P ; \lambda)$ via (7.248), we obtain the promised result (7.196) for the Seifert loop class on $\mathcal{N}(P)$, albeit in the special case $n=0$,

$$
\begin{aligned}
\left.W_{R}(C)\right|_{\mathcal{N}(P)} & =\mathrm{q}_{*} S_{\alpha}(\mathrm{u}) \\
& =\frac{1}{|\mathfrak{W}|} \operatorname{ch}_{R}\left(\mathcal{V}_{p}\right) \cdot \int_{\mathcal{O}_{-\lambda}} \eta \\
& =\operatorname{ch}_{R}\left(\mathcal{V}_{p}\right)
\end{aligned}
$$

In passing to the last line of (7.252), we see that integral of $\eta$ over $\mathcal{O}_{-\lambda}$ precisely cancels the factor $1 /|\mathfrak{W}|$ which appears under the anti-symmetrization in $(7.250)$.

Equivalently via (7.227),

$$
Z(\epsilon ; C, R)=\int_{\mathcal{N}(P)} \operatorname{ch}_{R}\left(\mathcal{V}_{p}\right) \cdot \exp \left[k \Omega_{0}\right] \cdot \operatorname{Td}(\mathcal{N}(P)) .
$$

To tie up a loose end, let us quickly sketch a computation of the Euler character of $G / T$. One approach, valid for any compact simple Lie group, is to use the Bruhat decomposition of the complex group $G_{\mathbb{C}}$. In a nutshell (see Section 23.4 of [52] for details), the Bruhat decomposition of $G_{\mathbb{C}}$ provides a distinguished covering of $G / T$ by disjoint open affine cells, each cell being labeled by an element of the Weyl group $\mathfrak{W}$ of $G$. As a concrete example, if $G=S U(2), \mathfrak{W} \cong \mathbb{Z}_{2}$, and $G / T=\mathbb{C P}^{1}$, the Bruhat decomposition implies 
the cellular presentation $\mathbb{C P}^{1}=\{p t\} \cup D^{2}$, where $D^{2}$ is the open disc realizing the complement of the given point. Since each Bruhat cell generally has even dimension, the Euler character simply counts the number of cells, which is given by $|\mathfrak{W}|$.

For the special case $G=S U(r+1)$, the Euler character of $G / T$ can also be computed by more elementary means. For each rank $r \geq 1$, we have the standard fiber bundle

$$
S U(r) \longrightarrow S U(r+1)
$$

Dividing $S U(r+1)$ in (7.254) by the maximal torus $T=U(1)^{r}$, we obtain

$$
\begin{aligned}
S U(r) / U(1)^{r-1} \longrightarrow & S U(r+1) / U(1)^{r} \\
& S^{2 r+1} / U(1) \cong \mathbb{C P}^{r} .
\end{aligned}
$$

Because we are about to compute the Euler character inductively in $r$, let us set

$$
X_{r}=S U(r) / U(1)^{r-1} .
$$

The fibration in (7.255), together with the multiplicativity of the Euler character, then implies

$$
\begin{aligned}
\chi_{\mathrm{top}}\left(X_{r+1}\right) & =\chi_{\mathrm{top}}\left(X_{r}\right) \cdot \chi_{\mathrm{top}}\left(\mathbb{C P}^{r}\right) \\
& =\chi_{\mathrm{top}}\left(X_{r}\right) \cdot(r+1)
\end{aligned}
$$

Trivially, $X_{1}$ is a point, so that $\chi_{\text {top }}\left(X_{1}\right)=1$. The relation in (7.257) thus yields by induction

$$
\chi_{\text {top }}\left(X_{r+1}\right)=(r+1) !=|\mathfrak{W}|, \quad G=S U(r+1) .
$$

\subsubsection{A symplectic model for localization on $\mathcal{M}_{0}(C, \alpha)$}

The identification (7.252) of the Seifert loop class with the character $\operatorname{ch}_{R}\left(\mathcal{V}_{p}\right)$ is certainly an elegant result, but one might wonder whether this identification is somehow special to Chern-Simons theory on the product $S^{1} \times \Sigma$. After all, only in this case does the Seifert loop path integral $Z(\epsilon ; C, R)$ compute a quantity as simple as the dimension of a Hilbert space, 
an observation which provided the starting point (7.211) for the preceding analysis. Indeed, given the intricacies of that analysis, one might question whether the Seifert loop operator still reduces to the character $\operatorname{ch}_{R}\left(\mathcal{V}_{p}\right)$ even when $M$ is the total space of a non-trivial $S^{1}$-bundle over $\Sigma$ of positive degree $n \geq 1$.

To complete our analysis of Chern-Simons gauge theory on Seifert manifolds, we now establish the universal description (7.196) of the Seifert loop class as $\operatorname{ch}_{R}\left(\mathcal{V}_{p}\right)$ for all degrees $n \geq 1$. In essence, our strategy is direct we apply the general non-abelian localization formula in (6.31) to the Seifert loop path integral on $M$. Rather than plunge immediately into details, however, let us quickly indicate the structure of that localization computation, which has two main steps.

According to our discussion of the classical Seifert loop operator in Section 7.1, the Seifert loop path integral localizes onto the extended moduli space $\mathcal{M}(C, \alpha)$ of flat connections on $M$ with fixed monodromy around the meridian of $C$, as in (7.10). For the first step in our computation, we use the localization formula in (6.31) to reduce the Seifert loop path integral over the infinite-dimensional space $\overline{\mathcal{A}}_{\alpha}=\overline{\mathcal{A}} \times \epsilon L \mathcal{O}_{\alpha}$ to the integral of an appropriate de Rham cohomology class $[d \mu]$ on each smooth component $\mathcal{M}_{0}(C, \alpha)$ of the moduli space $\mathcal{M}(C, \alpha)$. Schematically,

$$
\left.Z(\epsilon ; C, R)\right|_{\mathcal{M}_{0}(C, \alpha)}=\int_{\mathcal{M}_{0}(C, \alpha)}[d \mu], \quad[d \mu] \in H^{*}\left(\mathcal{M}_{0}(C, \alpha)\right)
$$

where the class $[d \mu]$ generally depends upon the discrete parameters $(n, k, \alpha)$ which specify respectively the degree of the $S^{1}$-bundle, the Chern-Simons level, and the highest weight of the irreducible representation $R$. As will hopefully be clear, the computation of $[d \mu]$ closely resembles the localization computation on $\mathcal{O}_{\alpha}$ in Section 7.2.

According to (7.196), the universal identification of the Seifert loop class with the character $\operatorname{ch}_{R}\left(\mathcal{V}_{p}\right)$ is to hold on each smooth component $\mathcal{M}_{0}$ of the basic moduli space $\mathcal{M}$ of flat connections, as opposed to the extended moduli space $\mathcal{M}(C, \alpha)$. For the second step in our calculation, we therefore push the class $[d \mu]$ on $\mathcal{M}_{0}(C, \alpha)$ down to $\mathcal{M}_{0}$ via the map q : $\mathcal{M}_{0}(C, \alpha) \rightarrow \mathcal{M}_{0}$ in the symplectic fibration (7.22) of moduli spaces. Not surprisingly, given the identifications

$$
\mathcal{M}_{0} \cong \widetilde{\mathcal{N}}(P), \quad \mathcal{M}_{0}(C, \alpha) \cong \widetilde{\mathcal{N}}(P ; \lambda), \quad \lambda=\frac{\alpha}{k},
$$

the pushdown under $\mathrm{q}_{*}$ proceeds along exactly the same lines as the previous index theory computation for the Seifert loop operator on $M=S^{1} \times \Sigma$. So 
for the second step in the computation, in which we push $[d \mu]$ down to $\mathcal{M}_{0}$, we effectively recycle the results of Jeffrey in [65].

Of the two main steps in our calculation of the Seifert loop class, only the first step, the localization computation of $[d \mu]$ on $\mathcal{M}_{0}(C, \alpha)$, requires additional work.

To make use of the general formula in (6.31) for localization on $\mathcal{M}_{0}(C, \alpha)$, we must first specify the symplectic model for a small neighborhood in $\overline{\mathcal{A}}_{\alpha}=\overline{\mathcal{A}} \times \epsilon L \mathcal{O}_{\alpha}$ of any pair $\left(A_{\mathrm{cl}}, U_{\mathrm{cl}}\right)$ which satisfies the classical equations of motion in (7.9) and thereby represents a point in $\mathcal{M}_{0}(C, \alpha)$. Since we assume $\mathcal{M}_{0}(C, \alpha)$ to be smooth, $A_{\mathrm{cl}}$ is an irreducible flat connection on the knot complement $M^{o}=M-C$, and the only gauge transformations, which fix the pair $\left(A_{\mathrm{cl}}, U_{\mathrm{cl}}\right)$ are constant gauge transformations taking values in the center $\mathcal{Z}(G)=\mathbb{Z}_{r+1}$ of the simply connected gauge group $G=\mathrm{SU}(r+1)$. Of course, here we note that the center of $G$ always acts trivially in the adjoint representation.

Without loss, we additionally assume that the pair $\left(A_{\mathrm{cl}}, U_{\mathrm{cl}}\right)$ is invariant under the Seifert action by $U(1)_{\mathrm{R}}$ on $M$. Hence the covariant derivative determined by $A_{\mathrm{cl}}$ commutes with the Lie derivative $£_{\mathrm{R}}$,

$$
\left[d_{A_{\mathrm{cl}}}, £_{\mathrm{R}}\right]=0
$$

and the fluctuating modes of the fields $(A, U)$ about the basepoint $\left(A_{\mathrm{cl}}, U_{\mathrm{cl}}\right)$ admit a covariant Fourier decomposition with respect to $U(1)_{R}$. For the defect field $U$, which we mostly focus on here, we will make the covariant Fourier decomposition explicit in a moment.

Following the ansatz in Section 6.2, we again model an equivariant neighborhood $N$ of the point $\left(A_{\mathrm{cl}}, U_{\mathrm{cl}}\right) \in \overline{\mathcal{A}}_{\alpha}$ on a symplectic fibration of the general form

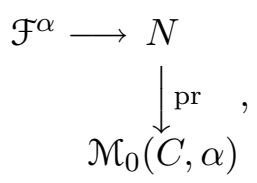

where $\mathcal{F}^{\alpha}$ is the total space of a homogeneous vector bundle over a quotient $\mathcal{H} / H_{0}^{\alpha}$,

$$
\mathcal{F}^{\alpha}=\mathcal{H} \times_{H_{0}^{\alpha}}\left(\mathfrak{h}^{\perp} \oplus \mathcal{E}_{1}^{\alpha}\right), \quad \mathfrak{h}^{\perp} \equiv \mathfrak{h} \ominus \mathfrak{h}_{0}^{\alpha} \ominus \mathcal{E}_{0}^{\alpha}
$$


Here $H_{0}^{\alpha}$ is the stabilizer of the point $\left(A_{\mathrm{cl}}, U_{\mathrm{cl}}\right)$ in the full Hamiltonian group

$$
\mathcal{H}=U(1)_{\mathrm{R}} \ltimes \widetilde{\mathcal{G}}_{0},
$$

and $\left(\mathcal{E}_{0}^{\alpha}, \mathcal{E}_{1}^{\alpha}\right)$ are vector spaces upon which $H_{0}^{\alpha}$ acts.

As in Section 7.2, we are left to identify $H_{0}^{\alpha}, \mathcal{E}_{0}^{\alpha}$, and $\mathcal{E}_{1}^{\alpha}$ in the canonical local model (7.263) for $\mathcal{F}^{\alpha}$.

First, since the pair $\left(A_{\mathrm{cl}}, U_{\mathrm{cl}}\right)$ is irreducible and invariant under $U(1)_{\mathrm{R}}$, the stabilizer $H_{0}^{\alpha} \subset \mathcal{H}$ is given immediately by the product

$$
H_{0}^{\alpha}=U(1)_{\mathrm{R}} \times \mathcal{Z}(G) \times U(1)_{\mathrm{z}} \equiv H_{0},
$$

where we recall that the central $U(1)_{\mathrm{z}} \subset \widetilde{\mathcal{G}}_{0}$ acts trivially on all of $\overline{\mathcal{A}}_{\alpha}$. As indicated in (7.265), because $H_{0}^{\alpha}$ does not in fact depend upon $\alpha$ (so long as $\alpha>0$ is regular), we abbreviate $H_{0}^{\alpha} \equiv H_{0}$ throughout the following analysis.

As for the vector spaces $\left(\mathcal{E}_{0}^{\alpha}, \mathcal{E}_{1}^{\alpha}\right)$, we proceed in close analogy to the discussion surrounding the similar identifications in (7.87) and (7.94) of Section 7.2. Since we consider a neighborhood of $\left(A_{\mathrm{cl}}, U_{\mathrm{cl}}\right)$ in the product $\overline{\mathcal{A}}_{\alpha}=\overline{\mathcal{A}} \times \epsilon L \mathcal{O}_{\alpha}$, both $\mathcal{E}_{0}^{\alpha}$ and $\mathcal{E}_{1}^{\alpha}$ decompose a priori into pair of subspaces, one subspace associated to the affine space $\overline{\mathcal{A}}$ and one subspace associated to the loopspace $L \mathcal{O}_{\alpha}$. In the case $\alpha=0$, for which $L \mathcal{O}_{\alpha}$ reduces to a point, we have already determined in Section 5.1 of [18] the summands associated to $\overline{\mathcal{A}}$ alone,

$$
\begin{aligned}
& \mathcal{E}_{0}=\bigoplus_{t \geq 1} H_{\frac{0}{\partial}}\left(\Sigma, \operatorname{ad}(P) \otimes\left(\mathcal{L}^{t} \oplus \mathcal{L}^{-t}\right)\right), \\
& \mathcal{E}_{1}=\bigoplus_{t \geq 1} H_{\frac{1}{\partial}}\left(\Sigma, \operatorname{ad}(P) \otimes\left(\mathcal{L}^{t} \oplus \mathcal{L}^{-t}\right)\right) .
\end{aligned}
$$

The pair $\left(\mathcal{E}_{0}, \mathcal{E}_{1}\right)$ in (7.266) should be compared to the corresponding pair in (7.84), for which we have merely replaced the Lie algebra $\mathfrak{g}$ of $G$ with the adjoint bundle $\operatorname{ad}(P)$ over $\Sigma$. As throughout, $P$ is the principal $G_{\text {ad }^{-}}$ bundle appearing in the diffeomorphism $\mathcal{M}_{0} \cong \tilde{\mathcal{N}}(P)$, and $\mathcal{L}$ is the positive line bundle over $\Sigma$ whose unit-circle bundle describes the Seifert manifold $M$.

Given (7.266), our job here is only to capture the additional symplectic geometry in $\mathcal{F}^{\alpha}$, which derives from the loopspace $L \mathcal{O}_{\alpha}$. As in Section 7.2, we identify the tangent space to $L \mathcal{O}_{\alpha}$ at the point $\left[U_{\mathrm{cl}}\right]$ with the space of 
"ATMP-17-1-A1-BEA" — 2013/5/15 — 19:32 — page 204 — \#204

maps $\delta U: C \rightarrow \mathfrak{g} \ominus \mathfrak{t}$, and again we decompose $\delta U$ into eigenmodes of $£_{\mathrm{R}}$ as

$$
\delta U=\sum_{t=-\infty}^{+\infty} \delta U_{t}, \quad £_{\mathrm{R}} \delta U_{t}=-2 \pi \mathrm{i} t \cdot \delta U_{t}
$$

Here, in identifying each eigenmode $\delta U_{t}$ geometrically with a map from $C$ to $\mathfrak{g} \ominus \mathfrak{t}$, we trivialize both the line bundle $\mathcal{L}$ and the adjoint bundle $\operatorname{ad}(P)$ at the basepoint $p \in \Sigma$ under the Seifert fiber $C \subset M$. For this reason, and in contrast to the description of $\left(\mathcal{E}_{0}, \mathcal{E}_{1}\right)$ in $(7.266)$, neither the adjoint bundle $\operatorname{ad}(P)$ nor the line bundle $\mathcal{L}$ play any role in (7.267).

Now, in the description (7.87) of $\mathcal{E}_{0}^{\alpha}$ in Section 7.2 , we were careful to include as an additional summand a copy of $\mathfrak{g}^{(1,0)}$ to account for directions tangent to the orbit $\mathcal{O}_{\alpha} \subset L \mathcal{O}_{\alpha}$ parameterizing constant loops. However for the case at hand, $\mathcal{O}_{\alpha}$ already appears (up to normalization) as a submanifold of the extended moduli space $\mathcal{M}_{0}(C, \alpha)$ sitting at the base of the symplectic fibration in (7.262). Thus for localization on $\mathcal{M}_{0}(C, \alpha)$, we do not need to account separately for $\mathcal{O}_{\alpha}$ in the vector space $\mathcal{E}_{0}^{\alpha}$. With nothing further to include, $\mathcal{E}_{0}^{\alpha}$ is given simply by

$$
\mathcal{E}_{0}^{\alpha}=\mathcal{E}_{0}, \quad \alpha>0 \quad \text { regular }
$$

Otherwise, via the Fourier decomposition in (7.267), the normal directions to $\mathcal{O}_{\alpha}$ inside $L \mathcal{O}_{\alpha}$ are given by a countable sum of copies of $\mathfrak{g} \ominus \mathfrak{t}$ graded by the non-zero integer $t \neq 0$. As in (7.91), we introduce the holomorphic normal bundle $\mathcal{N}_{\alpha}$ for the embedding $\mathcal{O}_{\alpha} \subset L \mathcal{O}_{\alpha}$,

$$
\mathcal{N}_{\alpha}=\bigoplus_{t \geq 1}\left[\mathfrak{g}_{t}^{(1,0)} \oplus \mathfrak{g}_{-t}^{(1,0)}\right]
$$

Once more, in the process of identifying $\mathcal{E}_{1}^{\alpha}$, we must be careful about the complex structure on $\mathcal{N}_{\alpha}$. Repeating verbatim the discussion which surrounds the corresponding presentation of $\mathcal{E}_{1}^{\alpha}$ in (7.94), we see that consistency with the convention in (6.23) requires that we identify $\mathcal{E}_{1}^{\alpha}$ as the direct sum of $\mathcal{E}_{1}$ in (7.266) with the conjugate normal bundle

$$
\overline{\mathcal{N}}_{\alpha}=\bigoplus_{t \geq 1}\left[\mathfrak{g}_{t}^{(0,1)} \oplus \mathfrak{g}_{-t}^{(0,1)}\right]
$$

Hence

$$
\mathcal{E}_{1}^{\alpha}=\mathcal{E}_{1} \oplus \overline{\mathcal{N}}_{\alpha}
$$


"ATMP-17-1-A1-BEA" — 2013/5/15 - 19:32 — page $205-\# 205$

LOCALIZATION FOR WILSON LOOPS IN CHERN-SIMONS

Together, (7.265), (7.268), and (7.271) specify the symplectic model for localization on $\mathcal{M}_{0}(C, \alpha)$.

\subsubsection{Non-abelian localization on $\mathcal{M}_{0}(C, \alpha)$}

We now possess all the ingredients required to apply the non-abelian localization formula in (6.31) to compute the cohomology class $[d \mu]$ in (7.259).

Immediately,

$$
\begin{aligned}
\left.Z(\epsilon ; C, R)\right|_{\mathcal{M}_{0}(C, \alpha)}= & \frac{(2 \pi \epsilon)}{|\mathcal{Z}(G)|} \int_{\mathfrak{h}_{0} \times \mathcal{M}_{0}(C, \alpha)}\left[\frac{d p}{2 \pi}\right]\left[\frac{d a}{2 \pi}\right] \frac{e_{H_{0}}\left(\mathcal{M}_{0}(C, \alpha), \mathcal{E}_{0}^{\alpha}\right)}{e_{H_{0}}\left(\mathcal{M}_{0}(C, \alpha), \mathcal{E}_{1}^{\alpha}\right)} \\
& \times \exp \left[\Omega_{\lambda}+\mathrm{i} \epsilon n \Theta-\mathrm{i} a+\mathrm{i} \epsilon p a\right] .
\end{aligned}
$$

Here the prefactor involving $\epsilon$ arises for the same reason as the corresponding prefactor in (7.95). Otherwise, the semiclassical contribution to $Z(\epsilon ; C, R)$ from $\mathcal{M}_{0}(C, \alpha)$ reduces to an integral over the abelian Lie algebra $\mathfrak{h}_{0} \cong \mathbb{R} \oplus \mathbb{R}$ of the stabilizer $H_{0}$, as well as an integral over $\mathcal{M}_{0}(C, \alpha)$ itself. Following the notation in Section 3, we parameterize $\mathfrak{h}_{0}$ with coordinates $(p, a)$.

As for the integrand in (7.272), beyond the ratio of equivariant Euler classes associated to $\left(\mathcal{E}_{0}^{\alpha}, \mathcal{E}_{1}^{\alpha}\right)$, we recognize in the argument of the exponential the equivariant symplectic form $\left[\Omega_{\lambda}-\mathrm{i} a\right]$ on $\mathcal{M}_{0}(C, \alpha)$, as appears more generally in the first line of (6.35). Similarly, $[n \Theta+p a]$ is the equivariant characteristic class of degree four on $\mathcal{M}_{0}(C, \alpha)$ appearing in the second line of (6.35). The same degree-four class enters the localization computation in Section 5.3 of [18], and according to the discussion there, $\Theta$ is given concretely in terms of the Chern roots $u$ of $\mathcal{V}_{p}$ by

$$
\Theta=-\frac{1}{2}(\mathfrak{u}, \mathfrak{u}) \in H^{4}\left(\mathcal{M}_{0}(C, \alpha)\right) .
$$

Since $\Theta$ is manifestly a symmetric function of the Chern roots, $\Theta$ is the pullback from $\mathcal{M}_{0}$ of a corresponding degree-four characteristic class of the universal bundle $\mathcal{V}_{p}$. Again, we find it convenient to abuse notation slightly, and we will not usually attempt to distinguish $\Theta$ as a characteristic class on $\mathcal{M}_{0}$ from its pullback (7.273) to the splitting manifold $\mathcal{M}_{0}(C, \alpha)$.

The attentive reader may note that $\Theta$ is multiplied in (7.272) by a factor $+i n$. The $+i$ arises trivially from the $+i$ that multiplies the Chern-Simons action in the original path integral. Otherwise, as we observed previously 
in (5.110) of [18], the degree $n$ arises from the geometric identity

$$
\int_{M} \kappa \wedge d \kappa \operatorname{Tr}\left(\phi^{2}\right)=n \int_{\Sigma} \omega \operatorname{Tr}\left(\phi^{2}\right)
$$

where $\phi$ is any element in the Lie algebra of $\mathcal{G}_{0}$, which pulls back from $\Sigma$, and $\omega$ is a unit-volume symplectic form on $\Sigma$ satisfying $d \kappa=n \pi^{*}(\omega)$. Under localization on $\mathcal{M}_{0} \cong \widetilde{\mathcal{N}}(P)$, the quadratic function of $\phi$ on the right of (7.274) reduces to $n \Theta$, from which the factor of $n$ in (7.272) arises.

As in Section 7.2, our main task here is to evaluate the ratio of equivariant Euler classes associated to the bundles $\left(\mathcal{E}_{0}^{\alpha}, \mathcal{E}_{1}^{\alpha}\right)$ over $\mathcal{M}_{0}(C, \alpha)$. Using the multiplicative property of the Euler class and the identification $\mathcal{E}_{1}^{\alpha}=\mathcal{E}_{1} \oplus \overline{\mathcal{N}}_{\alpha}$ in (7.271), we immediately factor the ratio in (7.272) as

$$
\frac{e_{H_{0}}\left(\mathcal{M}_{0}(C, \alpha), \mathcal{E}_{0}^{\alpha}\right)}{e_{H_{0}}\left(\mathcal{M}_{0}(C, \alpha), \mathcal{E}_{1}^{\alpha}\right)}=\mathrm{q}^{*}\left[\frac{e_{H_{0}}\left(\mathcal{M}_{0}, \mathcal{E}_{0}\right)}{e_{H_{0}}\left(\mathcal{M}_{0}, \mathcal{E}_{1}\right)}\right] \cdot \frac{1}{e_{H_{0}}\left(\mathcal{M}_{0}(C, \alpha), \overline{\mathcal{N}}_{\alpha}\right)}
$$

In obtaining (7.275), we observe that $\mathcal{E}_{0}$ and $\mathcal{E}_{1}$ are defined in (7.266) as equivariant bundles on $\mathcal{M}_{0}$ which pull back to $\mathcal{M}_{0}(C, \alpha)$, implying that the ratio of Euler classes pulls back as well.

Evaluating the ratio of equivariant Euler classes associated to $\mathcal{E}_{0}$ and $\mathcal{E}_{1}$ on $\mathcal{M}_{0}$ turns out to be fairly tricky. Luckily, in (5.168) of [18] we have already computed that ratio. For sake of brevity, we merely state the answer,

$$
\begin{aligned}
\frac{e_{H_{0}}\left(\mathcal{M}_{0}, \mathcal{E}_{0}\right)}{e_{H_{0}}\left(\mathcal{M}_{0}, \mathcal{E}_{1}\right)}= & \exp \left[-\frac{\mathrm{i} \pi}{2} \eta_{0}(0)+\frac{\pi}{p} c_{1}\left(\mathcal{M}_{0}\right)+\frac{\mathrm{i} n \check{c}_{\mathfrak{g}}}{2 \pi p^{2}} \Theta\right] \\
& \times \prod_{j=1}^{\operatorname{dim}_{\mathbb{C}} \mathcal{M}_{0}} \frac{\varpi_{j}}{2 \sinh \left(\pi \varpi_{j} / p\right)} \\
\eta_{0}(0)= & -\frac{n \Delta_{G}}{6}
\end{aligned}
$$

Here $\varpi_{j}$ for $j=1, \ldots, \operatorname{dim}_{\mathbb{C}} \mathcal{M}_{0}$ are the Chern roots of the complex tangent bundle of $\mathcal{M}_{0}$, so that

$$
c\left(\mathcal{M}_{0}\right)=\prod_{j=1}^{\operatorname{dim}_{\mathbb{C}} \mathcal{M}_{0}}\left(1+\varpi_{j}\right), \quad c_{1}\left(\mathcal{M}_{0}\right)=\sum_{j=1}^{\operatorname{dim}_{\mathbb{C}} \mathcal{M}_{0}} \varpi_{j}
$$

and we recall from (7.108) the expression for the constant $\eta_{0}(0)$. 
Given (7.276), the only new calculation required is to evaluate the equivariant Euler class of the bundle $\overline{\mathcal{N}}_{\alpha}$ in (7.270) as it fibers equivariantly over $\mathcal{M}_{0}(C, \alpha)$. According to the general description in (6.39), the equivariant Euler class of $\overline{\mathcal{N}}_{\alpha}$ is given by the formal product

$$
e_{H_{0}}\left(\mathcal{M}_{0}(C, \alpha), \overline{\mathcal{N}}_{\alpha}\right)=\prod_{t \neq 0} \prod_{\beta>0}(-i t p+\langle\beta, \mathfrak{u}\rangle)
$$

Here, the dependence on $p$ arises exactly as for the corresponding determinant in (7.117). Otherwise, as $\beta>0$ ranges over the positive roots of $G$, the two-forms $\langle\beta, \mathfrak{u}\rangle$ represent the Chern roots of each summand $\mathfrak{g}_{t}^{(0,1)} \subset \mathcal{N}_{\alpha}$ as it fibers over $\mathcal{M}_{0}(C, \alpha)$. Not coincidentally, we encountered the same Chern roots in the index theory computation on $M=S^{1} \times \Sigma$, as in (7.242) and (7.243).

To make sense of the infinite product in (7.278), we must regularize it in some way. See the remarks beginning at (5.150) in [18] for a general discussion of the ways in which (7.278) can be reasonably defined. For sake of time, we proceed here in a more ad hoc fashion and simply use zeta/eta-function regularization to define the respective norm and phase of the product in (7.278).

Thankfully, the evaluation of the product in (7.278) using zeta/etafunction regularization is also a calculation which we have, in effect, already accomplished. In (7.117) of Section 7.2, we encountered a product which is formally identical to the product in (7.278), provided we make the natural Chern-Weil substitution

$$
\phi \longmapsto 2 \pi \mathrm{i} u
$$

The computation of the equivariant Euler class of the bundle $\overline{\mathcal{N}}_{\alpha}$ then proceeds along exactly the same lines which led previously to the formula in (7.131). So either by a direct computation completely analogous to the computation following (7.117) (which we omit), or just by substituting $2 \pi \mathrm{i} u$ for $\phi$ in our previous result (7.131), we obtain

$$
e_{H_{0}}\left(\mathcal{N}_{0}(C, \alpha), \overline{\mathcal{N}}_{\alpha}\right)=\exp \left(-\frac{2 \pi\langle\rho, \mathfrak{u}\rangle}{p}\right) \cdot \prod_{\beta>0} \frac{2}{\langle\beta, \mathfrak{u}\rangle} \sinh \left(\frac{\pi\langle\beta, \mathfrak{u}\rangle}{p}\right),
$$

where $\rho$ is the usual Weyl vector. 
Combining the formulae in (7.276) and (7.280), we see that the ratio of equivariant Euler classes in (7.275) becomes

$$
\begin{aligned}
& \frac{e_{H_{0}}\left(\mathcal{M}_{0}(C, \alpha), \mathcal{E}_{0}^{\alpha}\right)}{e_{H_{0}}\left(\mathcal{M}_{0}(C, \alpha), \mathcal{E}_{1}^{\alpha}\right)} \\
& =\exp \left(-\frac{\mathrm{i} \pi}{2} \eta_{0}(0)+\frac{2 \pi\langle\rho, \mathfrak{u}\rangle}{p}\right) \cdot \prod_{\beta>0} \frac{\langle\beta, \mathfrak{u}\rangle}{2 \sinh (\pi\langle\beta, \mathfrak{u}\rangle / p)} \\
& \quad \times \mathrm{q}^{*}\left[\exp \left(\frac{\pi}{p} c_{1}\left(\mathcal{M}_{0}\right)+\frac{\mathrm{i} n \check{c}_{\mathfrak{g}}}{2 \pi p^{2}} \Theta\right) \cdot \prod_{j=1}^{\operatorname{dim}_{\mathbb{C}} \mathcal{M}_{0}} \frac{\varpi_{j}}{2 \sinh \left(\pi \varpi_{j} / p\right)}\right] .
\end{aligned}
$$

Of particular note, the ratio in (7.281) depends only on the coordinate $p$, not $a$, in the Lie algebra $\mathfrak{h}_{0} \cong \mathbb{R} \oplus \mathbb{R}$. We could have predicted this occurrence at the outset, since $a$ parameterizes the Lie algebra of the central $U(1)_{z}$ which acts trivially on $\overline{\mathcal{A}}_{\alpha}$. Just as in Section 7.2 , the integral over $a$ in (7.272) then yields a delta-function $2 \pi \delta(1-\epsilon p)$, and the integral over the remaining coordinate $p$ amounts to setting $p=1 / \epsilon$ in (7.281).

Thus we express $\left.Z(\epsilon ; C, R)\right|_{\mathcal{M}_{0}(C, \alpha)}$ solely as an integral over the classical Seifert loop moduli space $\mathcal{M}_{0}(C, \alpha)$,

$$
\begin{aligned}
\left.Z(\epsilon ; C, R)\right|_{\mathcal{M}_{0}(C, \alpha)} & \frac{1}{|\mathcal{Z}(G)|} \exp \left(-\frac{i \pi}{2} \eta_{0}(0)\right) \\
& \times \int_{\mathcal{M}_{0}(C, \alpha)} \exp (2 \pi \epsilon\langle\alpha+\rho, \mathfrak{u}\rangle) \cdot \prod_{\beta>0} \frac{\langle\beta, \mathfrak{u}\rangle}{2 \sinh (\pi \epsilon\langle\beta, \mathfrak{u}\rangle)} \\
& \times \mathrm{q}^{*}\left[\exp \left(\Omega+\pi \epsilon c_{1}\left(\mathcal{M}_{0}\right)+i \epsilon n\left(1+\frac{\epsilon \check{c}_{\mathfrak{g}}}{2 \pi}\right) \Theta\right) \cdot \prod_{j=1}^{\operatorname{dim}_{\mathbb{C}} \mathcal{M}_{0}} \frac{\varpi_{j}}{2 \sinh \left(\pi \epsilon \varpi_{j}\right)}\right] .
\end{aligned}
$$

Since we eventually want to push the integrand in (7.282) down to $\mathcal{M}_{0}$, we have been careful to factor out those classes which manifestly pull back from $\mathcal{M}_{0}$ under $\mathrm{q}^{*}$. In particular, when obtaining (7.282), we have used the symplectic decomposition

$$
\Omega_{\lambda}=\mathrm{q}^{*} \Omega+2 \pi \epsilon\langle\alpha, \mathfrak{u}\rangle, \quad \lambda=\alpha / k,
$$

as follows directly from (5.65) upon setting $\epsilon=2 \pi / k$. 
To make the cohomological interpretation of (7.282) more transparent, let us rescale each element in the cohomology ring of $\mathcal{M}_{0}(C, \alpha)$ by a factor $(2 \pi \epsilon)^{-q / 2}$, where $q$ is the degree of the given class. For instance, the Chern roots $\varpi_{j}$ and $u$, each of degree two, scale by

$$
\begin{gathered}
\varpi_{j} \longmapsto \frac{1}{2 \pi \epsilon} \varpi_{j}, \\
\mathfrak{u} \longmapsto \frac{1}{2 \pi \epsilon} \mathfrak{u} .
\end{gathered}
$$

To preserve the value of the integral over $\mathcal{M}_{0}(C, \alpha)$, we simultaneously scale the integral itself by an overall factor $(2 \pi \epsilon)^{d}$, where $d=\operatorname{dim}_{\mathbb{C}} \mathcal{M}_{0}(C, \alpha)$. After this change of variables to clear away extraneous factors of $\epsilon$, $\left.Z(\epsilon ; C, R)\right|_{\mathcal{M}_{0}(C, \alpha)}$ becomes

$$
\begin{aligned}
& \left.Z(\epsilon ; C, R)\right|_{\mathcal{M}_{0}(C, \alpha)} \\
& =\frac{1}{|\mathcal{Z}(G)|} \exp \left(-\frac{\mathrm{i} \pi}{2} \eta_{0}(0)\right) \int_{\mathcal{M}_{0}(C, \alpha)} \frac{\mathrm{e}^{\langle\alpha+\rho, \mathrm{u}\rangle}}{\mathrm{A}_{\rho}(\mathrm{u})} \prod_{\beta>0}\langle\beta, \mathrm{u}\rangle \\
& \quad \times \mathrm{q}^{*}\left[\exp \left(\frac{1}{2 \pi \epsilon} \Omega+\frac{1}{2} c_{1}\left(\mathcal{M}_{0}\right)+\mathrm{i} \frac{n}{4 \pi^{2} \epsilon_{\mathrm{r}}} \Theta\right) \widehat{A}\left(\mathcal{M}_{0}\right)\right],
\end{aligned}
$$

where we recall the definition of the renormalized coupling

$$
\epsilon_{\mathrm{r}}=\frac{2 \pi}{k+\check{c}_{\mathfrak{g}}} .
$$

The expression for $\left.Z(\epsilon ; C, R)\right|_{\mathcal{M}_{0}(C, \alpha)}$ in (7.285) deserves a number of comments. First, we recognize in the integral over $\mathcal{M}_{0}(C, \alpha)$ our friend the Weyl denominator $\mathrm{A}_{\rho}(\mathrm{u})$,

$$
\mathrm{A}_{\rho}(\mathfrak{u})=\prod_{\beta>0} 2 \sinh \left(\frac{\langle\beta, \mathfrak{u}\rangle}{2}\right)
$$

Second, we see that the integrand of (7.285) contains the $\widehat{A}$-genus of $\mathcal{M}_{0}$. In general, if $X$ is a smooth complex manifold, the $\widehat{A}$-genus of $X$ is given by the following product over the Chern roots $x_{j}$ for $j=1, \ldots, \operatorname{dim}_{\mathbb{C}} X$ of the complex tangent bundle $T X$,

$$
\widehat{A}(X)=\prod_{j=1}^{\operatorname{dim}_{\mathbb{C}} X} \frac{x_{j}}{2 \sinh \left(x_{j} / 2\right)} .
$$


"ATMP-17-1-A1-BEA" — 2013/5/15 — 19:32 — page $210 — \# 210$

Directly upon specialization,

$$
\widehat{A}\left(\mathcal{M}_{0}\right)=\prod_{j=1}^{\operatorname{dim}_{\mathbb{C}} \mathcal{M}_{0}} \frac{\varpi_{j}}{2 \sinh \left(\varpi_{j} / 2\right)}
$$

The alert reader may also recognize the $\widehat{A}$-genus of the orbit $\mathcal{O}_{-\lambda}$ (with $\lambda=\alpha / k)$ appearing as a factor in (7.285),

$$
\widehat{A}\left(\mathcal{O}_{-\lambda}\right)=\frac{1}{\mathrm{~A}_{\rho}(\mathrm{u})} \cdot \prod_{\beta>0}\langle\beta, \mathrm{u}\rangle
$$

In comparing the general definition (7.288) of the $\widehat{A}$-genus to (7.290), we recall that the classes $\langle\beta, \mathfrak{u}\rangle$ as $\beta>0$ ranges over the positive roots of $G$ are precisely the Chern roots of $\mathcal{O}_{-\lambda}$.

The appearance of the $\widehat{A}$-genus of the orbit $\mathcal{O}_{-\lambda}$ in $(7.285)$ is no accident. Just as in (7.197), we have a holomorphic fibration of complex manifolds

$$
\underset{\mathcal{N}_{-\lambda} \longrightarrow}{\underset{\mathcal{M}_{0}}{\mathcal{M}_{0}},} \quad \lambda=\frac{\alpha}{k} .
$$

The description of the $\widehat{A}$-genus in (7.288), like the description of the Todd class in (7.223), is manifestly multiplicative. Hence the fibration of $\mathcal{M}_{0}(C, \alpha)$ over $\mathcal{M}_{0}$ in (7.291) implies the relation

$$
\widehat{A}\left(\mathcal{M}_{0}(C, \alpha)\right)=\widehat{A}\left(\mathcal{O}_{-\lambda}\right) \cdot \widehat{A}\left(\mathcal{M}_{0}\right)
$$

The individual factors in (7.289) and (7.290) therefore combine in the integrand of $(7.285)$ to describe the $\widehat{A}$-genus of the extended moduli space $\mathcal{M}_{0}(C, \alpha)$.

Given the secret appearance of $\widehat{A}\left(\mathcal{M}_{0}(C, \alpha)\right)$ in the integrand of $\left.Z(\epsilon ; C, R)\right|_{\mathcal{M}_{0}(C, \alpha)}$, let us work backwards a bit to express the entire integral over $\mathcal{M}_{0}(C, \alpha)$ in terms of natural classes on the extended moduli space. In addition to the identities in (7.283) and (7.292), we note that the first Chern 
class of $\mathcal{M}_{0}(C, \alpha)$ is given by the sum

$$
\begin{aligned}
c_{1}\left(\mathcal{M}_{0}(C, \alpha)\right) & =\mathrm{q}^{*} c_{1}\left(\mathcal{M}_{0}\right)+c_{1}\left(\mathcal{O}_{-\lambda}\right), \\
& =\mathrm{q}^{*} c_{1}\left(\mathcal{M}_{0}\right)+\sum_{\beta>0}\langle\beta, \mathfrak{u}\rangle=\mathrm{q}^{*} c_{1}\left(\mathcal{M}_{0}\right)+2\langle\rho, \mathfrak{u}\rangle,
\end{aligned}
$$

as enters the argument of the exponentials in (7.285). Using (7.283), (7.292), and (7.293), we then rewrite $\left.Z(\epsilon ; C, R)\right|_{\mathcal{M}_{0}(C, \alpha)}$ in terms of classes defined intrinsically on $\mathcal{M}_{0}(C, \alpha)$,

$$
\begin{aligned}
\left.Z(\epsilon ; C, R)\right|_{\mathcal{M}_{0}(C, \alpha)}= & \frac{1}{|\mathcal{Z}(G)|} \exp \left(-\frac{\mathrm{i} \pi}{2} \eta_{0}(0)\right) \int_{\mathcal{M}_{0}(C, \alpha)} \widehat{A}\left(\mathcal{M}_{0}(C, \alpha)\right) \\
& \times \exp \left[\frac{1}{2 \pi \epsilon} \Omega_{\lambda}+\frac{1}{2} c_{1}\left(\mathcal{M}_{0}(C, \alpha)\right)+\mathrm{i} \frac{n}{4 \pi^{2} \epsilon_{\mathrm{r}}} \mathrm{q}^{*} \Theta\right] .
\end{aligned}
$$

We emphasize above that the degree-four characteristic class $\Theta$ pulls back from $\mathcal{M}_{0}$ under the map q : $\mathcal{M}_{0}(C, \alpha) \rightarrow \mathcal{M}_{0}$.

The integral over $\mathcal{M}_{0}(C, \alpha)$ in (7.294) should be compared to the expression for the partition function $\left.Z(\epsilon)\right|_{\mathcal{M}_{0}}$ in (5.172) of [18],

$$
\begin{aligned}
\left.Z(\epsilon)\right|_{\mathcal{M}_{0}}= & \frac{1}{|\mathcal{Z}(G)|} \exp \left(-\frac{\mathrm{i} \pi}{2} \eta_{0}(0)\right) \int_{\mathcal{M}_{0}} \widehat{A}\left(\mathcal{M}_{0}\right) \\
& \times \exp \left[\frac{1}{2 \pi \epsilon} \Omega+\frac{1}{2} c_{1}\left(\mathcal{M}_{0}\right)+\mathrm{i} \frac{n}{4 \pi^{2} \epsilon_{\mathrm{r}}} \Theta\right] .
\end{aligned}
$$

Just as for the cohomological formulae in (7.224), we see that the respective contributions from the extended moduli space $\mathcal{M}_{0}(C, \alpha)$ to $Z(\epsilon ; C, R)$ and from the basic moduli space $\mathcal{M}_{0}$ to $Z(\epsilon)$ are structurally identical, insofar as the same characteristic classes appear in the respective integrands of each. In hindsight, the beautiful correlation between (7.294) and (7.295) was bound to occur, since in both cases we apply the same localization formula in the same smooth setting. The structural agreement between (7.294) and (7.295) therefore provides an additional check on our computations.

Finally, returning our attention to (7.285), we note that the renormalized coupling $\epsilon_{\mathrm{r}}$ naturally appears in the coefficient of $\Theta$ when we rescale the integral in (7.282). Indeed, based upon our experience with Chern-Simons theory, we expect the integrand in (7.285) to depend on the Chern-Simons level $k$ only through the renormalized coupling $\epsilon_{\mathrm{r}}$, as opposed to the bare coupling $\epsilon$. 
To recast our result (7.285) entirely in terms of $\epsilon_{\mathrm{r}}$, we apply a theorem of Drezet and Narasimhan [44] which determines $c_{1}\left(\mathcal{M}_{0}\right)$ in the case $G=S U(r+1)$ to be

$$
c_{1}\left(\mathcal{M}_{0}\right)=2(r+1) \Omega_{0}, \quad \Omega_{0}=\frac{1}{4 \pi^{2}} \Omega .
$$

Since $\check{c}_{\mathfrak{g}}=r+1$ as well, the local contribution from $\mathcal{M}_{0}(C, \alpha)$ to $Z(\epsilon ; C, R)$ becomes

$$
\begin{aligned}
\left.Z(\epsilon ; C, R)\right|_{\mathcal{M}_{0}(C, \alpha)}= & \frac{1}{|\mathcal{Z}(G)|} \exp \left(-\frac{\mathrm{i} \pi}{2} \eta_{0}(0)\right) \int_{\mathcal{M}_{0}(C, \alpha)} \frac{\mathrm{e}^{\langle\alpha+\rho, \mathfrak{u}\rangle}}{\mathrm{A}_{\rho}(\mathrm{u})} \prod_{\beta>0}\langle\beta, \mathrm{u}\rangle \\
& \times \mathrm{q}^{*}\left[\exp \left(\frac{1}{2 \pi \epsilon_{\mathrm{r}}}\left(\Omega+\mathrm{i} \frac{n}{2 \pi} \Theta\right)\right) \cdot \widehat{A}\left(\mathcal{M}_{0}\right)\right]
\end{aligned}
$$

and all dependence on $k$ has been absorbed into the renormalized coupling $\epsilon_{\mathrm{r}}$.

According to (7.259), the integrand in (7.297) is the class $[d \mu] \in$ $H^{*}\left(\mathcal{M}_{0}(C, \alpha)\right)$ which describes the local contribution from $\mathcal{M}_{0}(C, \alpha)$ to the Seifert loop path integral $Z(\epsilon ; C, R)$,

$$
\begin{aligned}
{[d \mu]=} & \frac{1}{|\mathcal{Z}(G)|} \exp \left(-\frac{\mathrm{i} \pi}{2} \eta_{0}(0)\right) \\
& \times \frac{\mathrm{e}^{\langle\alpha+\rho, \mathfrak{u}\rangle}}{\mathrm{A}_{\rho}(\mathrm{u})} \cdot \prod_{\beta>0}\langle\beta, \mathfrak{u}\rangle \cdot \mathrm{q}^{*}\left[\exp \left(\frac{1}{2 \pi \epsilon_{\mathrm{r}}}\left(\Omega+\mathrm{i} \frac{n}{2 \pi} \Theta\right)\right) \cdot \widehat{A}\left(\mathcal{M}_{0}\right)\right] .
\end{aligned}
$$

To obtain a cohomological formula for the Seifert loop class itself, we are left to push $[d \mu]$ down to $\mathcal{M}_{0}$ via the map q : $\mathcal{M}_{0}(C, \alpha) \rightarrow \mathcal{M}_{0}$ in (7.291).

\subsubsection{Pushdown to $\mathcal{M}_{0}$ and relation to index theory}

Like the pair of expressions in (7.294) and (7.295), the integral over $\mathcal{M}_{0}(C, \alpha)$ in (7.297) should be compared to the corresponding localization result for the partition function $\left.Z(\epsilon)\right|_{\mathcal{M}_{0}}$ in (5.174) of [18],

$$
\left.Z(\epsilon)\right|_{\mathcal{M}_{0}}=\frac{1}{|\mathcal{Z}(G)|} \exp \left(-\frac{\mathrm{i} \pi}{2} \eta_{0}(0)\right) \int_{\mathcal{M}_{0}} \exp \left[\frac{1}{2 \pi \epsilon_{\mathrm{r}}}\left(\Omega+\mathrm{i} \frac{n}{2 \pi} \Theta\right)\right] \cdot \widehat{A}\left(\mathcal{M}_{0}\right) \text {. }
$$

The Seifert loop class $\left.W_{R}(C)\right|_{\mathcal{M}_{0}}$ is then the element of $H^{*}\left(\mathcal{M}_{0}\right)$ such that the pushdown $\mathrm{q}_{*}[d \mu]$ is given by the product of $\left.W_{R}(C)\right|_{\mathcal{M}_{0}}$ with the integrand 
of the partition function $\left.Z(\epsilon)\right|_{\mathcal{M}_{0}}$ in (7.299), such that

$$
\begin{aligned}
\left.Z(\epsilon ; C, R)\right|_{\mathcal{M}_{0}}= & \frac{1}{|\mathcal{Z}(G)|} \exp \left(-\frac{\mathrm{i} \pi}{2} \eta_{0}(0)\right) \\
& \times\left.\int_{\mathcal{M}_{0}} W_{R}(C)\right|_{\mathcal{M}_{0}} \cdot \exp \left[\frac{1}{2 \pi \epsilon_{\mathrm{r}}}\left(\Omega+i \frac{n}{2 \pi} \Theta\right)\right] \cdot \widehat{A}\left(\mathcal{M}_{0}\right) .
\end{aligned}
$$

Comparing the Seifert integrand $[d \mu] \in H^{*}\left(\mathcal{M}_{0}(C, \alpha)\right)$ in (7.298) to the preceding formula $(7.300)$ for $\left.Z(\epsilon ; C, R)\right|_{\mathcal{M}_{0}}$, we deduce

$$
\left.W_{R}(C)\right|_{\mathcal{M}_{0}}=\mathrm{q}_{*} S_{\alpha}(\mathfrak{u}), \quad S_{\alpha}(\mathfrak{u})=\frac{\mathrm{e}^{\langle\alpha+\rho, \mathfrak{u}\rangle}}{\mathrm{A}_{\rho}(\mathfrak{u})} \cdot \prod_{\beta>0}\langle\beta, \mathfrak{u}\rangle .
$$

The class $S_{\alpha}(u)$ in (7.301) is precisely the same class that appeared previously in (7.244) when we considered the Seifert loop operator in ChernSimons theory on $S^{1} \times \Sigma$. Moreover, under the identification of moduli spaces $\mathcal{M}_{0}(C, \alpha) \cong \widetilde{\mathcal{N}}(P ; \lambda)$ and $\mathcal{M}_{0} \cong \widetilde{\mathcal{N}}(P)$, the calculation of the pushdown $\mathrm{q}_{*} S_{\alpha}(\mathrm{u})$ proceeds just as before. So without further ado, recycling the result in (7.252), we find the promised general description for the Seifert loop class,

$$
\left.W_{R}(C)\right|_{\mathcal{M}_{0}}=\operatorname{ch}_{R}\left(\mathcal{V}_{p}\right)
$$

Equivalently,

$$
\begin{aligned}
\left.Z(\epsilon ; C, R)\right|_{\mathcal{M}_{0}}= & \frac{1}{|\mathcal{Z}(G)|} \exp \left(-\frac{\mathrm{i} \pi}{2} \eta_{0}(0)\right) \int_{\mathcal{M}_{0}} \operatorname{ch}_{R}\left(\mathcal{V}_{p}\right) \\
& \times \exp \left[\frac{1}{2 \pi \epsilon_{\mathrm{r}}}\left(\Omega+\mathrm{i} \frac{n}{2 \pi} \Theta\right)\right] \cdot \widehat{A}\left(\mathcal{M}_{0}\right) .
\end{aligned}
$$

The expression for $\left.Z(\epsilon ; C, R)\right|_{\mathcal{M}_{0}}$ in (7.303) is very similar to the cohomological formula (7.253) derived from the index theorem when $M=S^{1} \times \Sigma$. To make the relation to (7.253) more transparent, we recall that the Todd class and the $\widehat{A}$-genus of a complex manifold $X$ generally satisfy the relation

$$
\operatorname{Td}(X)=\exp \left[\frac{1}{2} c_{1}(X)\right] \cdot \widehat{A}(X)
$$

as follows directly by comparison of the formulae in (7.223) and (7.288). This identity, applied to $\widehat{A}\left(\mathcal{M}_{0}\right)$ in (7.285), implies that the contribution 
from $\mathcal{M}_{0}$ to $Z(\epsilon ; C, R)$ can be alternatively presented as

$$
\begin{aligned}
\left.Z(\epsilon ; C, R)\right|_{\mathcal{M}_{0}}= & \frac{1}{|\mathcal{Z}(G)|} \exp \left(-\frac{\mathrm{i} \pi}{2} \eta_{0}(0)\right) \int_{\mathcal{M}_{0}} \operatorname{ch}_{R}\left(\mathcal{V}_{p}\right) \\
& \times \exp \left[k \Omega_{0}+\mathrm{i} \frac{n}{4 \pi^{2} \epsilon_{\mathrm{r}}} \Theta\right] \cdot \operatorname{Td}\left(\mathcal{M}_{0}\right) .
\end{aligned}
$$

Under the identification $\mathcal{M}_{0} \cong \widetilde{\mathcal{N}}(P)$, the integrand of (7.305) now manifestly reproduces the index density in (7.253) when $n=0$. Otherwise, if $n>0$, our localization formula for $\left.Z(\epsilon ; C, R)\right|_{\mathcal{M}_{0}}$ amounts to a surprisingly simple deformation away from the index theory result.

\subsubsection{The Yang-Mills limit of the Seifert loop operator}

Given the smooth Seifert fibration $S^{1} \stackrel{n}{\rightarrow} M \stackrel{\pi}{\rightarrow} \Sigma$ of degree $n>0$, another interesting regime in which to consider the Seifert loop path integral $Z(\epsilon ; C, R)$ is the limit $n \rightarrow \infty$. Intuitively, as $n$ becomes large, the nontrivial Fourier modes of the gauge field $A$ along the circle fiber of $M$ decouple, and Chern-Simons theory on $M$ effectively reduces to Yang-Mills theory on $\Sigma$. In this limit, according to the discussion surrounding (5.2), we expect the Seifert loop operator $W_{R}(C)$ to reduce to the Yang-Mills monodromy operator $\mathrm{V}_{\lambda}(p)$, where $\lambda=\alpha / k$.

To check the latter statement, let us quickly apply non-abelian localization to the monodromy operator path integral $Z\left(\epsilon_{\mathrm{ym}} ; p, \lambda\right)$. Here to avoid confusion with the Chern-Simons coupling parameter $\epsilon=2 \pi / k$, we introduce a distinct notation $\epsilon_{\mathrm{ym}}=g_{\mathrm{ym}}^{2}$ for the Yang-Mills coupling.

As we demonstrated in (5.19), $Z\left(\epsilon_{\mathrm{ym}} ; p, \lambda\right)$ takes the canonical form determined by the Hamiltonian action of $\mathcal{G}(P)$ on the symplectic space $\mathcal{A}(P)_{\lambda}=$ $\mathcal{A}(P) \times 2 \pi \mathcal{O}_{\lambda}$. When $\mathcal{G}(P)$ acts freely near the vanishing locus of the moment map $\mu=\mathcal{F}_{A}$ in $\mathcal{A}(P)_{\lambda}$, the extended moduli space $\mathcal{N}(P ; \lambda)$ is smooth, and the very simple version (6.40) of the non-abelian localization formula applies. Immediately by (6.40),

$$
\left.Z\left(\epsilon_{\mathrm{ym}} ; p, \lambda\right)\right|_{\mathcal{N}(P ; \lambda)}=\int_{\mathcal{N}(P ; \lambda)} \exp \left[\Omega_{\lambda}+\epsilon_{\mathrm{ym}} \Theta\right]
$$

Again, $\Omega_{\lambda}$ is the symplectic form on $\mathcal{N}(P ; \lambda)$ in (5.65), and $\Theta$ is the degreefour characteristic class of $\mathcal{V}_{p}$ in $(7.273)$.

If one wishes, the integrand in (7.306) can be pushed down to $\mathcal{N}(P)$ to describe the monodromy operator $\boldsymbol{V}_{\lambda}(p)$ as a class on $\mathcal{N}(P)$. For sake of 
"ATMP-17-1-A1-BEA" — 2013/5/15 - 19:32 — page 215 — \#215

brevity, we will not do so here. We refer the interested reader to [106] and to [21] for a more detailed analysis of $Z\left(\epsilon_{\mathrm{ym}} ; p, \lambda\right)$ in the special case $\epsilon_{\mathrm{ym}}=0$, for which the integral in (7.306) merely computes the symplectic volume of $\mathcal{N}(P ; \lambda)$.

We now compare our localization result for $Z(\epsilon ; C, R)$ in $(7.282)$ to the Yang-Mills formula in (7.306). To obtain a sensible limit for $Z(\epsilon ; C, R)$ as $n$ goes to infinity, we take the parameters $k, \alpha$ to infinity with the ratios $\epsilon_{\mathrm{ym}}=n / k$ and $\lambda=\alpha / k$ held fixed,

$$
n, k, \alpha \rightarrow \infty, \quad \epsilon_{\mathrm{ym}}=\frac{n}{k}, \quad \lambda=\frac{\alpha}{k} \quad \text { fixed }
$$

To obtain a finite result in the limit (7.307), we also scale $Z(\epsilon ; C, R)$ by an overall prefactor $(2 \pi \epsilon)^{d}$, where $d=\operatorname{dim}_{\mathbb{C}} \mathcal{M}_{0}(C, \alpha)$,

$$
Z(\epsilon ; C, R) \rightarrow(2 \pi \epsilon)^{d} Z(\epsilon ; C, R)
$$

Under this scaling, the products over the Chern roots $\langle\beta, u\rangle$ and $\varpi_{j}$ in (7.282) reduce to the identity, and the respective quantum shifts by $\check{c}_{\mathfrak{g}}$ and $\rho$ both vanish. With the final identification $\mathcal{M}_{0}(C, \alpha)=\widetilde{\mathcal{N}}(P ; \lambda)$, the localization formula for Chern-Simons theory in (7.282) then reproduces the Yang-Mills formula in (7.306), at least up to overall normalization.

\section{Acknowledgments}

I take great pleasure in thanking Dima Belov, Dan Freed, Amir-Kian Kashani-Poor, Martin Roček, Yongbin Ruan, Amit Sever, George Thompson, and Jonathan Weitsman for stimulating discussions on the matters herein. I also thank the organizers and participants of the Bonn workshop "Chern-Simons Gauge Theory: 20 Years After" and the 2007 Simons Workshop, where portions of this paper were presented. Last but not least, I thank Cliff Taubes for very helpful comments on a preliminary draft, and I especially thank Edward Witten, both for our prior collaboration on the subject and for posing the question which sparked this work. This work was supported in part under DOE grant no. DE-FG02-92ER40697. Any opinions, findings, and conclusions or recommendations expressed in this material are those of the author and do not necessarily reflect the views of the Department of Energy. 
"ATMP-17-1-A1-BEA" — 2013/5/15 — 19:32 — page 216 — \#216

\section{Appendix A Index of notation}

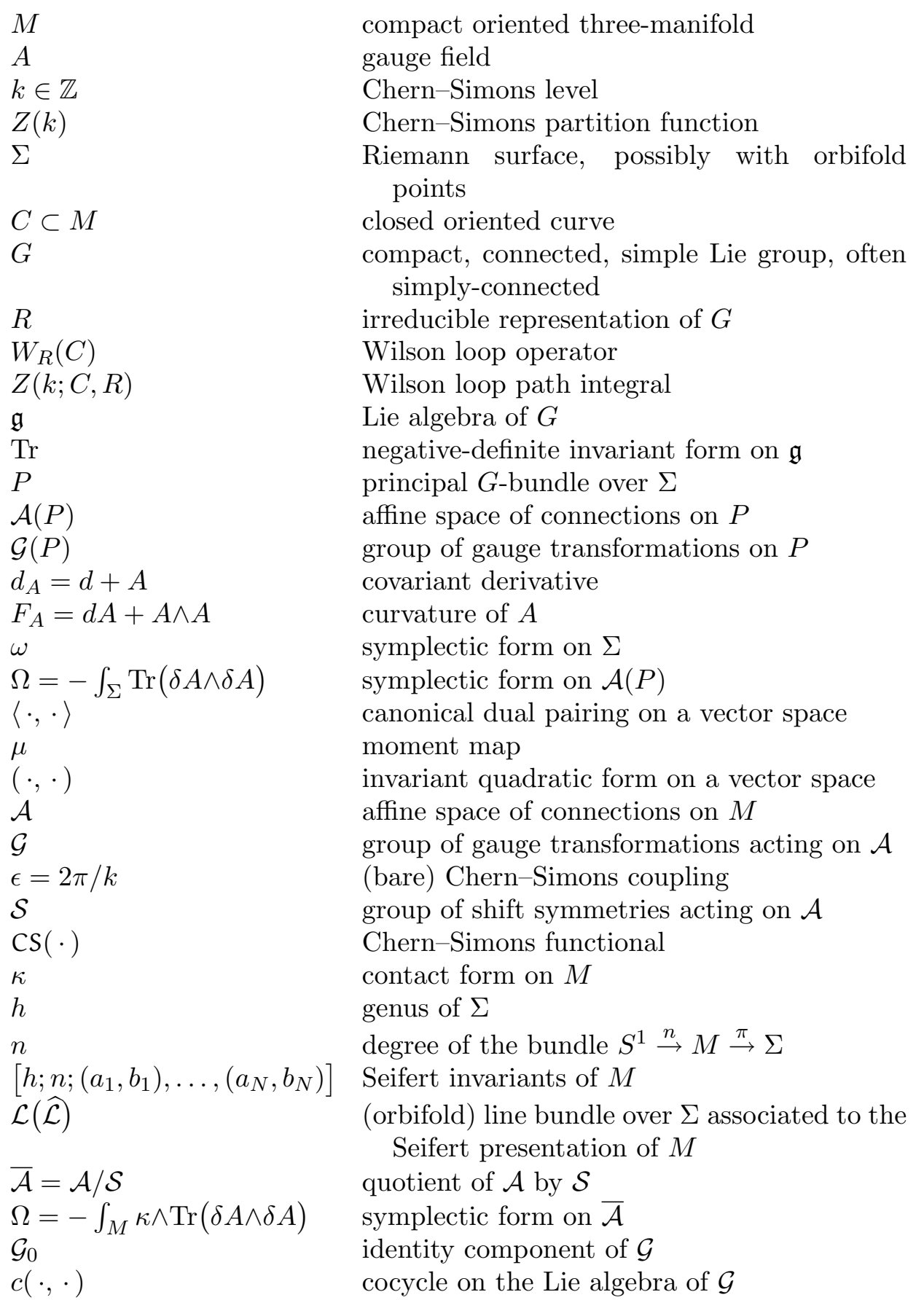




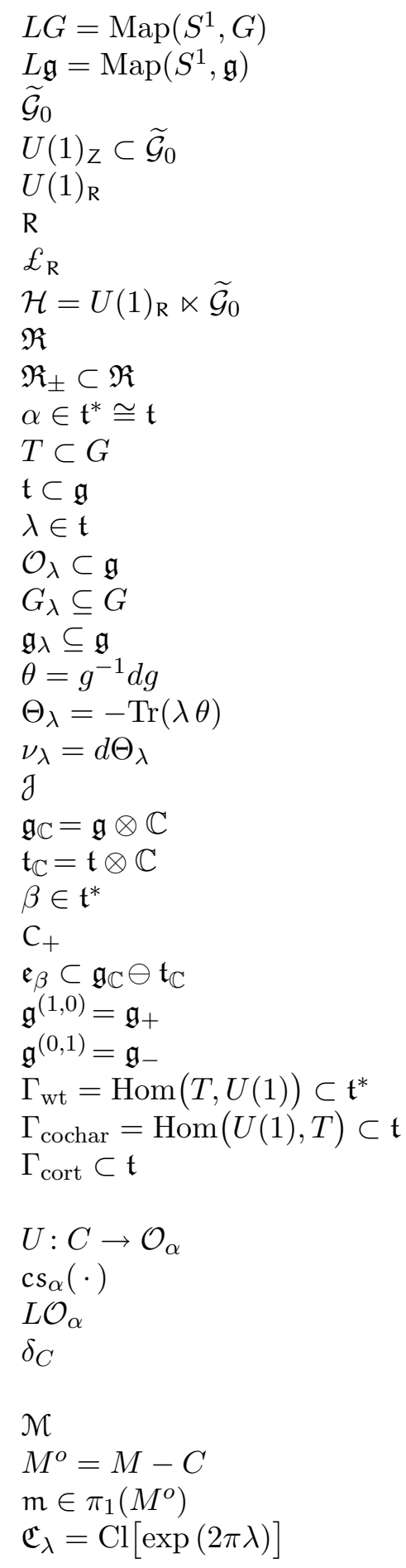

loop group of $G$

Lie algebra of $L G$

central extension of $\mathcal{G}_{0}$ defined by $c(\cdot, \cdot)$

central $U(1)$ subgroup of $\widetilde{\mathcal{G}}_{0}$

locally free, Seifert $U(1)$ acting on $M$

vector field on $M$ generating $U(1)_{\mathrm{R}}$

Lie derivative along $\mathrm{R}$

Hamiltonian group acting on $\overline{\mathcal{A}}$

set of roots of $G$

positive/negative roots of $G$

highest weight of the representation $R$

maximal torus

Cartan subalgebra

element of $\mathfrak{t}$

adjoint orbit through $\lambda$

stabilizer of $\lambda$

Lie algebra of $G_{\lambda}$

left-invariant Cartan form on $G$

pre-symplectic one-form on $G$

coadjoint symplectic form on $\mathcal{O}_{\lambda}$

invariant complex structure on $\mathcal{O}_{\lambda}$

complexification of $\mathfrak{g}$

complexification of $\mathfrak{t}$

root of $G$

positive Weyl chamber

rootspace associated to $\beta$

holomorphic tangent space to $\mathcal{O}_{\lambda}$

anti-holomorphic tangent space to $\mathcal{O}_{\lambda}$

weight lattice of $G$

cocharacter lattice of $G$

coroot lattice of $G$; identical to $\Gamma_{\text {cochar }}$ when $G$ is simply-connected

sigma model field on $C$ with target $\mathcal{O}_{\alpha}$

topological, Chern-Simons-type action for $U$

free (unbased) loopspace of $\mathcal{O}_{\alpha}$

two-form with delta-function support which is

Poincaré dual to $C \subset M$

moduli space of flat connections on $M$

complement of $C \subset M$

meridian of $C$

conjugacy class in $G$ containing $\Lambda=$ $\exp (2 \pi \lambda)$ 
$\mathcal{M}(C, \alpha)$

$\mathcal{F}_{A}=F_{A}+\epsilon\left(g \alpha g^{-1}\right) \delta_{C}$

$\overline{\mathcal{A}}_{\alpha}=\overline{\mathcal{A}} \times \epsilon L \mathcal{O}_{\alpha}$

$\Upsilon_{\alpha}$

$\Xi_{\alpha}$

$\Omega_{\alpha}=\Omega+\epsilon \Upsilon_{\alpha}$

$\mathrm{V}_{\lambda}(p)$

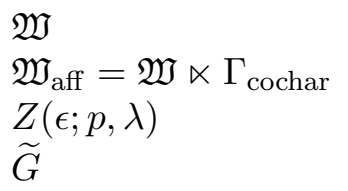

$\mathcal{Z}(\widetilde{G}), \mathcal{Z}(G)$

$\delta_{p}$

$\mathrm{D}_{+} \subset \mathrm{C}_{+}$

$\vartheta$

$\mathcal{A}(P)_{\lambda}=\mathcal{A}(P) \times \mathcal{O}_{2 \pi \lambda}$

$\Omega_{\lambda}=\Omega+2 \pi \nu_{\lambda}$

$\mathcal{N}(P)$

$\mathcal{N}(P ; \lambda) \equiv \mathcal{N}(P ; p, \lambda)$

$\Sigma^{o}=\Sigma-\{p\}$

$\varrho^{o}: \pi_{1}\left(\Sigma^{o}\right) \rightarrow G$

$\Sigma^{o o}=\Sigma-\{p\}-\{q\}$

$\varrho^{o o}: \pi_{1}\left(\Sigma^{\circ o}\right) \rightarrow G$

$\widetilde{\mathcal{N}}(P ; \lambda)$

$\Omega, \Omega_{\lambda}$

$e_{\lambda}$

$\mathcal{Z}_{\Lambda} \subseteq G$

V

$\nu_{p}$ moduli space of flat connections on $M^{o}$ with holonomy around $\mathrm{m}$ in $\mathfrak{C}_{\alpha / k}$

generalized curvature in the presence of $W_{R}(C)$

symplectic space associated to $Z(\epsilon ; C, R)$

coadjoint symplectic form on $L \mathcal{O}_{\alpha}$ induced from

$\nu_{\alpha}$

pre-symplectic one-form on $L G$ such that $\Upsilon_{\alpha}=\delta \Xi_{\alpha}$

symplectic form on $\overline{\mathcal{A}}_{\alpha}$

monodromy operator inserted at $p \in \Sigma$ with parameter $\lambda \in \mathfrak{t}$

Weyl group of $G$

affine Weyl group of $G$

monodromy operator path integral

simply-connected form of the Yang-Mills gauge group $G$

center of $\widetilde{G}$, respectively $G$

two-form with delta-function support which is Poincaré dual to $p \in \Sigma$

fundamental Weyl alcove

highest root of $G$

symplectic space associated to $Z(\epsilon ; p, \lambda)$

symplectic form on $\mathcal{A}(P)_{\lambda}$

moduli space of flat connections on the $G$-bundle $P$ over $\Sigma$

moduli space of flat connections on $P$ with monodromy at $p$ in $\mathfrak{C}_{\lambda}=\mathrm{Cl}[\exp (2 \pi \lambda)]$

punctured Riemann surface

homomorphism from the fundamental group of $\Sigma^{o}$ to $G$

doubly-punctured Riemann surface

homomorphism from the fundamental group of $\Sigma^{o o}$ to $G$

unramified, degree $|\widetilde{G}: G|^{2 h}$ cover of $\mathcal{N}(P ; \lambda)$

symplectic forms on $\mathcal{N}(P)$ and $\mathcal{N}(P ; \lambda)$ induced from those on $\mathcal{A}(P)$ and $\mathcal{A}(P)_{\lambda}$

closed two-form on $\mathcal{N}(P ; \lambda)$ which restricts fiberwise to $\nu_{\lambda}$ on $\mathcal{O}_{\lambda}$

centralizer of the element $\Lambda$ in $G$

universal bundle over $\Sigma \times \mathcal{N}(P)$

restriction of $\mathcal{V}$ to $\{p\} \times \mathcal{N}(P)$ for $p \in \Sigma$ 
$\mathcal{L}_{j}, j=1, \ldots, r+1 \quad$ splitting line for the pullback of $\mathcal{V}_{p}$ to $\mathcal{N}(P ; \lambda)$

$\mathrm{u}_{j}=c_{1}\left(\mathcal{L}_{j}\right)$

$\hat{\omega}_{1}, \ldots, \hat{\omega}_{r+1}$

Chern root of $\mathcal{V}_{p}$

standard generators for the weight lattice of $S U(r+1)$, with $\hat{\omega}_{1}+\cdots+\hat{\omega}_{r+1}=0$

$\mathbf{u}=i \operatorname{diag}\left(\mathfrak{u}_{1}, \cdots, \mathfrak{u}_{r+1}\right)$

element of $H^{2}(\mathcal{N}(P ; \lambda) ; \mathbb{Z}) \otimes \mathfrak{t}$ encoding the

Chern roots of $\mathcal{V}_{p}$

$X$

symplectic manifold

$\Omega$

symplectic form on $X$

$H$

connected Lie group acting in a Hamiltonian fashion on $X$

$\Delta_{H}$

$\mathfrak{h}$

(real) dimension of $H$

$\mathfrak{h}^{*}$

$\mu: X \rightarrow \mathfrak{h}^{*}$

$\epsilon \in \mathbb{R}$

$S=\frac{1}{2}(\mu, \mu)$

$V(\phi)$

$D=d+i \iota_{V(\phi)}$

$H_{H}^{*}(X)$

Lie algebra of $H$

dual of $\mathfrak{h}$

moment map for $H$ acting on $X$

coupling parameter

norm-square of moment map

vector field on $X$ determined by action of $\phi \in \mathfrak{h}$

BRST operator/Cartan differential

$H$-equivariant cohomology ring of $X$ with coefficients in $\mathbb{R}$

$\mathbf{J}: T X \rightarrow T X$

almost-complex structure on $X$ compatible with $\Omega$

$\mathcal{C} \subset X$

connected component in the critical locus of $S=\frac{1}{2}(\mu, \mu)$ on $X$

$H_{0} \subset H$
$\mathfrak{h}_{0}$
$\mathcal{M}=\mathcal{C} / H$
$\gamma_{0} \in \mathfrak{h}_{0}^{*} \cong \mathfrak{h}_{0}$
$F=H \times_{H_{0}}\left(\mathfrak{h}^{\perp} \oplus E_{1}\right)$

stabilizer at an arbitrary basepoint on $\mathcal{C}$

Lie algebra of $H_{0}$

smooth quotient of $\mathcal{C}$ by $H$

value of the moment map $\mu$ at the basepoint on $\mathcal{C}$

symplectic fiber over $\mathcal{M}$ in a neighborhood of $\mathcal{C} \subset X$

$E_{0} \subset \mathfrak{h}$

$\mathfrak{h}^{\perp}=\mathfrak{h} \ominus \mathfrak{h}_{0} \ominus E_{0}$

$E_{1}$

subspace of $\mathfrak{h}$, preserved under the adjoint action of $\mathfrak{h}_{0}$, on which $\gamma_{0}$ acts non-degenerately

orthocomplement to $\mathfrak{h}_{0} \oplus E_{0}$ inside $\mathfrak{h}$

symplectic vector space on which $H_{0}$ acts in a Hamiltonian fashion and $\gamma_{0}$ acts nondegenerately

$\left.Z(\epsilon)\right|_{\mathcal{M}}$

$\Theta$

local contribution to $Z(\epsilon)$ from $\mathcal{C} \subset X$

degree-four characteristic class on $\mathcal{M}$ derived from $-\frac{1}{2}(\phi, \phi)$ under the Chern-Weil homomorphism 


\begin{tabular}{|c|c|}
\hline$e_{H_{0}}\left(\mathcal{M}, E_{0}\right), e_{H_{0}}\left(\mathcal{M}, E_{1}\right)$ & $\begin{array}{l}H_{0} \text {-equivariant Euler classes of the complex } \\
\text { vector bundles over } \mathcal{M} \text { associated to } E_{0} \text { and } \\
E_{1}\end{array}$ \\
\hline$\Delta_{G}=\operatorname{dim} G$ & dimension of $G$ \\
\hline$\Delta_{G_{\lambda}}=\operatorname{dim} G_{\lambda}$ & mension of $G_{\lambda} \subseteq G$ \\
\hline $\mathrm{a}_{\ell}, \mathrm{b}_{\ell}, \mathrm{c}_{j} \in \pi_{1}(M)$ & $\begin{array}{l}\text { generators derived from cycles in the orbifold } \\
\text { base } \Sigma \text { of the Seifert manifold } M\end{array}$ \\
\hline $\mathrm{f} \in \pi_{1}(M)$ & $\begin{array}{l}\text { generator associated to the circle fiber of the } \\
\text { Seifert manifold } M\end{array}$ \\
\hline$G_{\mathrm{ad}}=G / \mathcal{Z}(G)$ & mply-connected group $G$ \\
\hline $\mathcal{M}_{0}$ & 100 \\
\hline $\mathcal{M}_{0}(C, \alpha)$ & $\mathfrak{\imath}(C, \alpha)$ \\
\hline$q: \mathcal{N}(P ; \lambda) \rightarrow \mathcal{N}(P)$ & th moduli spaces \\
\hline $\mathrm{q}: \mathcal{M}_{0}(C, \alpha) \rightarrow \mathcal{M}_{0}$ & $\mathrm{mp}$ \\
\hline & er number of $\mathfrak{g}$ \\
\hline$\rho=\frac{1}{2} \sum_{\beta>0} \beta$ & Weyl vector of $G$ \\
\hline$d=\left|H_{1}(M)\right|$ & $\begin{array}{l}\text { order of } H_{1}(M) \text { when } M \text { is a rational homol- } \\
\text { ogy sphere }\end{array}$ \\
\hline$\varrho_{\mathrm{ab}}: \pi_{1}\left(M^{o}\right) \rightarrow G$ & $\begin{array}{l}\text { maximally reducible, abelian representation } \\
\text { of } \pi_{1}\left(M^{o}\right)\end{array}$ \\
\hline $\mathcal{K}_{\mathbf{p}, \mathbf{q}}$ & $(\mathbf{p}, \mathbf{q})$-torus knot in $S^{3}$ \\
\hline$\epsilon_{r}=2 \pi /\left(k+\check{c}_{\mathfrak{g}}\right)$ & alized Chern-Simons coupling \\
\hline $\begin{array}{l}\mathrm{P}=\prod_{j=1}^{N} a_{j} \\
s(b, a)\end{array}$ & $\begin{array}{l}\text { product of orders of orbifold points on } \Sigma \\
\text { Dedekind sum }\end{array}$ \\
\hline & $\begin{array}{l}\text { irreducible representation of } S U(2) \text { with } \\
\quad \text { dimension } j\end{array}$ \\
\hline $\operatorname{ch}_{\mathbf{j}}(z)=\sinh (j z) / \sinh (z)$ & character for the $S U(2)$ representation $\mathbf{j}$ \\
\hline $\mathcal{C}^{(0)}=\mathrm{e}^{\frac{i \pi}{4}} \times \mathbb{R}$ & diagonal contour through the origin \\
\hline $\mathcal{C}^{(l)}=\mathcal{C}^{(0)}-4 \pi i l(\mathrm{P} / d)$ & shiftec \\
\hline$\left.\operatorname{Res}(f(z))\right|_{z=z_{0}}$ & residue of the function $f(z)$ at $z=z_{0}$ \\
\hline$Z\left(\epsilon ; \mathcal{K}_{\mathbf{p}, \mathbf{q}}, \mathbf{j}\right)$ & $\begin{array}{l}\mathrm{SU}(2) \text { Wilson loop path integral for the torus } \\
\text { knot } \mathcal{K}_{\mathbf{p}, \mathbf{q}} \subset S^{3} \text { decorated with the repre- } \\
\text { sentation } \mathbf{j}\end{array}$ \\
\hline$\left.Z\left(\epsilon ; \mathcal{K}_{\mathbf{p}, \mathbf{q}}, \mathbf{j}\right)\right|_{\{0\}}$ & $\begin{array}{l}\text { contribution to } Z\left(\epsilon ; \mathcal{K}_{\mathbf{p}, \mathbf{q}}, \mathbf{j}\right) \text { from the trivial } \\
\quad \text { connection on } S^{3}\end{array}$ \\
\hline$Z\left(\epsilon ; \mathcal{K}_{\mathbf{p}, \mathbf{q}}, \mathbf{j}\right)_{\text {res }}$ & residue contribution to $Z\left(\epsilon ; \mathcal{K}_{\mathbf{p}, \mathbf{q}}, \mathbf{j}\right)$ \\
\hline$V_{\mathcal{K}}(\mathrm{t})$ & Jones polynomial of the knot $\mathcal{K}$ \\
\hline & \\
\hline
\end{tabular}




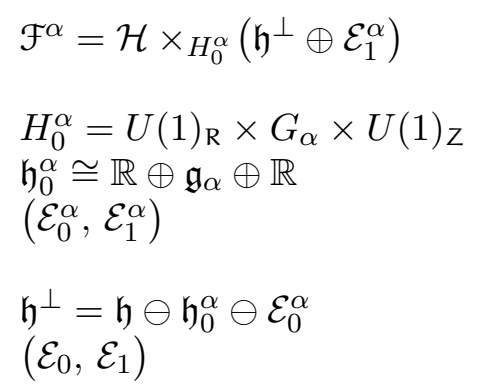

symplectic fiber describing a neighborhood of $\mathcal{O}_{\alpha} \subset \overline{\mathcal{A}}_{\alpha}$

stabilizer in $\mathcal{H}$ of a point in $\mathcal{O}_{\alpha}$

Lie algebra of $H_{0}^{\alpha}$

infinite-dimensional complex vector spaces which determine $\mathcal{F}^{\alpha}$

orthocomplement to $\mathfrak{h}_{0}^{\alpha} \oplus \mathcal{E}_{0}^{\alpha}$ inside $\mathfrak{h}$

infinite-dimensional complex vector spaces which describe a neighborhood of $\{0\} \in \overline{\mathcal{A}}$

$\mathcal{N}_{\alpha}=\bigoplus_{t \geq 1}\left[\mathfrak{g}_{t}^{(1,0)} \oplus \mathfrak{g}_{-t}^{(1,0)}\right]$

graded normal bundle to $\mathcal{O}_{\alpha}$ inside $L \mathcal{O}_{\alpha}$

$\overline{\mathcal{N}}_{\alpha}=\bigoplus_{t \geq 1}\left[\mathfrak{g}_{t}^{(0,1)} \oplus \mathfrak{g}_{-t}^{(0,1)}\right]$

conjugate of $\mathcal{N}_{\alpha}$

$\left.Z(\epsilon ; C, R)\right|_{\mathcal{O}_{\alpha} / G}$

$e(\overline{\mathcal{A}}), e\left(L \mathcal{O}_{\alpha}\right)$

local contribution to the Seifert loop path integral from the point $\left\{\varrho_{\mathrm{ab}}\right\} \cong \mathcal{O}_{\alpha} / G$

equivariant Euler classes associated to the normal directions to $\mathcal{O}_{\alpha}$ inside $\overline{\mathcal{A}} \times \epsilon L \mathcal{O}_{\alpha}$

$\eta_{0}(0)$

$\rho^{[\alpha]}=\frac{1}{2} \sum_{(\beta, \alpha)>0} \beta$

adiabatic eta-invariant on the Seifert manifold $M$

generalized Weyl vector

$\mathrm{A}_{\alpha}=\sum_{w \in \mathfrak{W}}(-1)^{w} \mathrm{e}^{w \cdot \alpha}$

alternating sum of exponentials

$\operatorname{ch}_{R}$

$\mathrm{B}_{\alpha}$

character of the representation $R$ of $G$

generalized Weyl denominator

Atiyah two-framing on $M$

Seifert two-framing on $M$

$\operatorname{lk}\left(C, C^{\prime}\right)$

linking number of curves $C$ and $C^{\prime}$ in the integral homology sphere $M$

$\mathbf{S}, \mathbf{T}$
$\mathcal{V}^{R}$
$\operatorname{ch}(E)$
$\mathcal{H}(k)$
$\mathcal{L}_{0}$
$\Omega_{0}=\Omega / 4 \pi^{2}$
$\mathcal{H}(k ; \alpha)$

standard generators of $S L(2, \mathbb{Z})$

associated universal bundle with fiber $R$ over $\Sigma \times \mathcal{N}(P)$

Chern character of a complex vector bundle E

Hilbert space of Chern-Simons theory at level $k$ on $\Sigma$

line bundle generating the Picard group of $\mathcal{N}(P)$

first Chern class of $\mathcal{L}_{0}$

Hilbert space of Chern-Simons theory at level $k$ with a Wilson line decorated by $\alpha$ puncturing $\Sigma$ 
$\mathcal{L}_{\alpha}^{(k)}$

$\chi(X, E)$

$\operatorname{Td}(X)$

$\chi_{\text {top }}$

$[d \mu] \in H^{*}\left(\mathcal{M}_{0}(C, \alpha)\right)$

$\Theta=-\frac{1}{2}(\mathrm{u}, \mathrm{u})$

$\widehat{A}(X)$

$\epsilon_{\mathrm{ym}}=g_{\mathrm{ym}}^{2}$ prequantum line bundle over $\mathcal{N}(P ; \lambda)$ for $\lambda=\alpha / k$

Euler character of a holomorphic vector bundle $E$ over a complex manifold $X$

Todd class of $X$

topological Euler character

de Rham class on $\mathcal{M}_{0}(C, \alpha)$ describing $\left.Z(\epsilon ; C, R)\right|_{\mathcal{M}_{0}(C, \alpha)}$ under localization

degree-four characteristic class of $\mathcal{V}_{p}$

$\widehat{A}$-genus of a complex manifold $X$

two-dimensional Yang-Mills coupling parameter

\section{Appendix B Vanishing lemma for a Gaussian sum}

In this appendix, we consider the Gaussian sum which appears in $Z\left(\epsilon ; \mathcal{K}_{\mathbf{p}, \mathbf{q}}\right.$, j) ${ }_{\text {res }}$ in (7.71),

$$
\begin{aligned}
I & =\sum_{t=1}^{2 \mathbf{p q}-1}(-1)^{t(j+1)} \sin \left(\frac{\pi t}{\mathbf{p}}\right) \sin \left(\frac{\pi t}{\mathbf{q}}\right) \exp \left(\frac{-\mathrm{i} \pi k_{r}}{2 \mathbf{p q}} t^{2}\right), \\
k_{r} & \equiv k+2 .
\end{aligned}
$$

We recall that $\mathbf{p}$ and $\mathbf{q}$ are positive, relatively prime integers which label the torus knot $\mathcal{K}_{\mathbf{p}, \mathbf{q}}$, and $j$ is the dimension of the irreducible $S U(2)$ representation $\mathbf{j}$.

Our goal is now to show that $I=0$. The proof is elementary, but as often the case with arithmetic arguments of this sort, the proof does require a tiny bit of work. For instance, $I$ need not vanish if $\operatorname{gcd}(\mathbf{p}, \mathbf{q})>1$, as one can easily verify in examples.

To start, since $\mathbf{p}$ and $\mathbf{q}$ are relatively-prime, there exist unique integers $\mathbf{r}$ and $\mathbf{s}$ such that

$$
\mathbf{p s}-\mathbf{q r}=1, \quad 0<\mathbf{s}<\mathbf{q}, \quad 0<\mathbf{r}<\mathbf{p},
$$

or

$$
\frac{\mathbf{s}}{\mathbf{q}}-\frac{\mathbf{r}}{\mathbf{p}}=\frac{1}{\mathbf{p q}} .
$$


Hence the sum in (B.1) can be immediately rewritten as

$$
I=\sum_{t=0}^{2 \mathbf{p q}-1}(-1)^{t(j+1)} \sin \left(\frac{\pi t}{\mathbf{p}}\right) \sin \left(\frac{\pi t}{\mathbf{q}}\right) \exp \left(\frac{-\mathrm{i} \pi k_{r} \mathbf{s}}{2 \mathbf{q}} t^{2}\right) \exp \left(\frac{\mathrm{i} \pi k_{r} \mathbf{r}}{2 \mathbf{p}} t^{2}\right)
$$

We include the summand for $t=0$ without loss (since that term trivially vanishes), and we note that the summand of (B.4) is otherwise manifestly periodic in $t$ with period 2pq.

Let us expand each sine in (B.4) as a sum of exponentials, so that

$$
I=I^{(++)}+I^{(--)}-I^{(+-)}-I^{(-+)},
$$

where

$I^{( \pm \pm)}=\sum_{t \in \mathbb{Z}_{2 \mathbf{p q}}}-\frac{1}{4}(-1)^{t(j+1)} \exp \left(\frac{-\mathrm{i} \pi k_{r} \mathbf{s}}{2 \mathbf{q}} t^{2} \pm \frac{\mathrm{i} \pi}{\mathbf{q}} t\right) \exp \left(\frac{\mathrm{i} \pi k_{r} \mathbf{r}}{2 \mathbf{p}} t^{2} \pm \frac{\mathrm{i} \pi}{\mathbf{p}} t\right)$.

As the summand in $I^{( \pm \pm)}$depends only on the value of $t$ modulo $2 \mathbf{p q}$, we regard the sum in (B.6) as running over elements of the cyclic group $\mathbb{Z}_{2 \mathbf{p q}}$. We note that trivially

$$
I^{(++)}=I^{(--)}, \quad I^{(+-)}=I^{(-+)},
$$

where these identifications follow by sending $t \mapsto-t$ in (B.6). To prove that $I$ vanishes, we will similarly demonstrate that

$$
I^{(++)}=I^{(+-)} .
$$

Our argument depends on the value of $k_{r} \bmod ^{42} \mathbf{p}$. Without loss, we assume that $\mathbf{p}>1$, since $I$ vanishes trivially if $\mathbf{p}=1$. We now consider two cases, depending upon whether or not $k_{r}$ is relatively-prime to $\mathbf{p}$.

Let us first assume that

$$
\operatorname{gcd}\left(k_{r}, \mathbf{p}\right)=d>1 \text {, }
$$

so that $\mathbf{p}$ and $k_{r}$ are not relatively-prime. We therefore introduce the reduced integer $\overline{\mathbf{p}}=\mathbf{p} / d$. On one hand, a overall shift $t \mapsto t+2 \overline{\mathbf{p}} \mathbf{q}$ in the

\footnotetext{
${ }^{42}$ The roles of $\mathbf{p}$ and $\mathbf{q}$ are entirely symmetric, but we will focus on $\mathbf{p}$ for concreteness.
} 
sum over elements of $\mathbb{Z}_{2 \text { pq }}$ trivially leaves $I^{( \pm \pm)}$invariant. On the other hand, under this shift the summand of (B.6) transforms with the phase $\zeta=\exp ( \pm 2 \pi \mathrm{i} \mathbf{q} / d)$, where the sign in $\zeta$ is determined by the sign in the term $\exp ( \pm \mathrm{i} \pi t / \mathbf{p})$ in (B.6). Thus

$$
I^{( \pm \pm)}=\zeta \cdot I^{( \pm \pm)}
$$

Finally, since $d$ divides $\mathbf{p}$ and $\operatorname{gcd}(\mathbf{p}, \mathbf{q})=1$, the integers $d$ and $\mathbf{q}$ must themselves be relatively-prime. Thus $\zeta \neq 1$, and B.10 implies that each of the four summands $I^{( \pm \pm)}$separately vanishes when (B.9) holds.

We are left to consider the case that $\mathbf{p}$ and $k_{r}$ are relatively prime,

$$
\operatorname{gcd}\left(k_{r}, \mathbf{p}\right)=1 \text {. }
$$

In this case, there again exist positive integers $a$ and $b$ such that

$$
a k_{r}-b \mathbf{p}=1, \quad 0<a<\mathbf{p}, \quad 0<b<k_{r} .
$$

That is, $a$ defines an inverse for $k_{r} \bmod \mathbf{p}$, so that $k_{r}^{-1} \equiv a \bmod \mathbf{p}$. Similarly, according to (B.2), $\mathbf{q}^{-1} \equiv-\mathbf{r} \bmod \mathbf{p}$.

We now consider shifting the summation variable $t$ in $I^{(++)}$by $t \mapsto t+$ $2 \mathbf{q} a$. Under this shift, the factors $(-1)^{t(j+1)}$ and $\exp \left[\left(-\mathrm{i} \pi k_{r} \mathbf{s} t^{2}+2 \pi \mathrm{i} t\right) / 2 \mathbf{q}\right]$ appearing in the summand of (B.6) are invariant, but

$$
\exp \left(\frac{\mathrm{i} \pi k_{r} \mathbf{r}}{2 \mathbf{p}} t^{2}+\frac{\mathrm{i} \pi}{\mathbf{p}} t\right) \longmapsto \zeta(t) \cdot \exp \left(\frac{\mathrm{i} \pi k_{r} \mathbf{r}}{2 \mathbf{p}} t^{2}+\frac{\mathrm{i} \pi}{\mathbf{p}} t\right)
$$

where

$$
\begin{aligned}
\zeta(t) & =\exp \left[\frac{2 \pi \mathrm{i}}{\mathbf{p}}\left(k_{r} \mathbf{r} \mathbf{q} a t+k_{r} \mathbf{r} \mathbf{q}^{2} a^{2}+\mathbf{q} a\right)\right] \\
& =\exp \left[-\frac{2 \pi \mathrm{i}}{\mathbf{p}} t\right]
\end{aligned}
$$

In passing to the second line of (B.14), we recall that $k_{r} a \equiv 1 \bmod \mathbf{p}$ and $\mathbf{q} \mathbf{r} \equiv-1 \bmod \mathbf{p}$. As a result,

$$
\begin{aligned}
& I^{(++)} \\
& =\sum_{t \in \mathbb{Z}_{2 \mathbf{p q}}}-\frac{1}{4}(-1)^{t(j+1)} \zeta(t) \exp \left(\frac{-\mathrm{i} \pi k_{r} \mathbf{s}}{2 \mathbf{q}} t^{2}+\frac{\mathrm{i} \pi}{\mathbf{q}} t\right) \exp \left(\frac{\mathrm{i} \pi k_{r} \mathbf{r}}{2 \mathbf{p}} t^{2}+\frac{\mathrm{i} \pi}{\mathbf{p}} t\right),
\end{aligned}
$$




$$
\begin{aligned}
& =\sum_{t \in \mathbb{Z}_{2 \mathbf{p q}}}-\frac{1}{4}(-1)^{t(j+1)} \exp \left(\frac{-\mathrm{i} \pi k_{r} \mathbf{s}}{2 \mathbf{q}} t^{2}+\frac{\mathrm{i} \pi}{\mathbf{q}} t\right) \exp \left(\frac{\mathrm{i} \pi k_{r} \mathbf{r}}{2 \mathbf{p}} t^{2}-\frac{\mathrm{i} \pi}{\mathbf{p}} t\right) \\
& =I^{(+-)} .
\end{aligned}
$$

So via (B.5) and (B.7), the Gaussian sum $I$ vanishes when $\mathbf{p}$ and $\mathbf{q}$ are relatively-prime.

\section{Appendix C The adiabatic eta-invariant on a Seifert manifold}

In this appendix, we explain how the adiabatic eta-invariant $\eta_{0}(0)$ is defined and computed on a general Seifert three-manifold $M$. Although the discussion here will be reasonably self-contained, it will also be only a sketch, and we refer the reader to Section 5.2 of [18] along with [89] for further details.

As usual for computations of functional determinants, we introduce an eta-function [14] to define the phase of the formal expression in (7.105),

$$
e(\overline{\mathcal{A}})=\operatorname{det}\left(\left.\frac{\psi}{2 \pi}\right|_{\mathcal{E}_{0}}\right) \operatorname{det}\left(\left.\frac{\psi}{2 \pi}\right|_{\mathcal{E}_{1}}\right)^{-1}, \quad \psi \equiv(p, \phi, a) \in \mathbb{R} \oplus \mathfrak{g}_{\alpha} \oplus \mathbb{R}
$$

Concretely, the generator $\psi$ acts on elements of both $\mathcal{E}_{0}$ and $\mathcal{E}_{1}$ as the firstorder differential operator ${ }^{43}$

$$
\mathcal{D}_{(p, \phi)}=p £_{\mathrm{R}}+[\phi, \cdot] .
$$

If $\mathcal{L}$ is the line bundle over $\Sigma$ associated to the Seifert manifold $M$, then $£_{\mathrm{R}}$ acts on sections of $\mathcal{L}^{t}$ with eigenvalue $-2 \pi \mathrm{i} t$. Hence $e(\overline{\mathcal{A}})$ can be written concretely as a product over the non-zero eigenvalues of $£_{\mathrm{R}}$ as

$$
e(\overline{\mathcal{A}})=\prod_{t \neq 0} \operatorname{det}\left[\left.\left(-\mathrm{i} t p+\frac{[\phi, \cdot]}{2 \pi}\right)\right|_{\mathfrak{g}}\right]^{\chi\left(\mathcal{L}^{t}\right)} .
$$

In (C.3) we correctly account for the ratio of determinants associated to $\mathcal{E}_{0}$ and $\mathcal{E}_{1}$ by introducing the Euler character

$$
\chi\left(\mathcal{L}^{t}\right)=\operatorname{dim}_{\mathbb{C}} H \frac{0}{\partial}\left(\Sigma, \mathcal{L}^{t}\right)-\operatorname{dim}_{\mathbb{C}} H \frac{1}{\partial}\left(\Sigma, \mathcal{L}^{t}\right) .
$$

\footnotetext{
${ }^{43}$ We note that the sign of $\phi$ in $\mathcal{D}_{(p, \phi)}$ is opposite to the corresponding sign in [18], a difference which can be traced to our convention in (2.5).
} 
Without loss, we take $\phi$ to lie in a Cartan subalgebra $\mathfrak{t} \subset \mathfrak{g}$. As standard, we diagonalize the adjoint action of $\phi$ on $\mathfrak{g}$ by introducing the roots $\beta \in \mathfrak{t}^{*}$. By definition, if $x_{\beta}$ is an element of the associated rootspace $\mathfrak{e}_{\beta} \subset \mathfrak{g}_{\mathbb{C}}$, then $\left[\phi, x_{\beta}\right]=i\langle\beta, \phi\rangle x_{\beta}$. Hence in terms of the roots $\beta$,

$$
\begin{aligned}
\left.\operatorname{det}\left(-\mathrm{i} t p+\frac{[\phi, \cdot]}{2 \pi}\right)\right|_{\mathfrak{g}} & =(-\mathrm{i} t p)^{\Delta_{G}} \prod_{\beta}\left(1-\frac{\langle\beta, \phi\rangle}{2 \pi t p}\right), \quad \Delta_{G}=\operatorname{dim} G \\
& =(-\mathrm{i} t p)^{\Delta_{G}} \prod_{\beta>0}\left(1-\left(\frac{\langle\beta, \phi\rangle}{2 \pi t p}\right)^{2}\right),
\end{aligned}
$$

where in the second line of (C.5) we rewrite the product over all roots as a product over only the positive roots $\beta>0$.

Using (C.5), we express $e(\overline{\mathcal{A}})$ as

$$
e(\overline{\mathcal{A}})=\exp \left(-\frac{\mathrm{i} \pi}{2} \eta\right) \cdot \prod_{t \geq 1}\left|(t p)^{\Delta_{G}} \prod_{\beta>0}\left(1-\left(\frac{\langle\beta, \phi\rangle}{2 \pi t p}\right)^{2}\right)\right|^{\chi\left(\mathcal{L}^{t}\right)+\chi\left(\mathcal{L}^{-t}\right)}
$$

In this appendix, we are most interested in the phase $\exp \left(-\frac{\mathrm{i} \pi}{2} \eta\right)$, which involves an infinite product of factors $\pm i$. The norm of $e(\overline{\mathcal{A}})$ is relatively straightforward to evaluate, as done in Section 5.2 of [18] using zeta-function regularization.

At least formally, the phase $\eta$ in (C.6) is given by the difference

$$
\eta \approx \sum_{\lambda_{(0)} \neq 0} \operatorname{sign}\left(\lambda_{(0)}\right)-\sum_{\lambda_{(1)} \neq 0} \operatorname{sign}\left(\lambda_{(1)}\right)
$$

where $\lambda_{(0)}$ and $\lambda_{(1)}$ range over the eigenvalues of the operator $\frac{1}{2 \pi i} \mathcal{D}_{(p, \phi)}$ acting respectively on $\mathcal{E}_{0}$ and $\mathcal{E}_{1}$. These eigenvalues are real, but they do not generally have a definite sign.

We are careful not to write (C.7) with an equality, because the sums appearing on the right in (C.7) are ill-defined without a regulator. To regulate these sums, we introduce the eta-function

$$
\eta_{(p, \phi)}(s)=\sum_{\lambda_{(0)} \neq 0} \operatorname{sign}\left(\lambda_{(0)}\right)\left|\lambda_{(0)}\right|^{-s}-\sum_{\lambda_{(1)} \neq 0} \operatorname{sign}\left(\lambda_{(1)}\right)\left|\lambda_{(1)}\right|^{-s} .
$$


Here, $s$ is a complex variable. When the real part of $s$ is sufficiently large, the sums in (C.8) are absolutely convergent, so that $\eta_{(p, \phi)}(s)$ is defined in that case. Otherwise, $\eta_{(p, \phi)}(s)$ is defined by analytic continuation in the $s$-plane. Assuming that the limit $s \rightarrow 0$ exists, we then set

$$
\eta=\eta_{(p, \phi)}(0)
$$

As shown in Section 5.2 of [18], $\eta_{(p, \phi)}(s)$ retains a non-trivial dependence on $p$ and $\phi$ even in the limit $s \rightarrow 0$, due to divergences in the naive sums in (C.7). Specifically,

$$
\eta_{(p, \phi)}(0)=\eta_{0}(0)-\frac{\check{c}_{\mathfrak{g}}}{2(\pi p)^{2}}\left(\frac{d}{P}\right) \operatorname{Tr}\left(\phi^{2}\right)
$$

where $\check{c}_{\mathfrak{g}}$ is the dual Coxeter number of the simply-laced Lie algebra $\mathfrak{g}$. The constant $\eta_{0}(0)$ in (C.10) is given by the value at $s=0$ of the $(p, \phi)$ independent eta-function

$$
\begin{aligned}
\eta_{0}(s) & =\sum_{t \neq 0} \sum_{\beta} \chi\left(\mathcal{L}^{t}\right) \operatorname{sign}(t)|t|^{-s} \\
& =\sum_{t \geq 1} \sum_{\beta} \frac{\chi\left(\mathcal{L}^{t}\right)-\chi\left(\mathcal{L}^{-t}\right)}{t^{s}}
\end{aligned}
$$

To remedy the small omission in [18], we now provide a general computation of $\eta_{0}(0)$, following the proof of Proposition 1.4 in [89].

\section{C.1 Evaluating $\eta_{0}(0)$}

By assumption, $\mathcal{L}$ is the orbifold line bundle, which describes the Seifert manifold $M$ with Seifert invariants

$$
\left[h ; n ;\left(a_{1}, b_{1}\right), \ldots,\left(a_{N}, b_{N}\right)\right], \quad \operatorname{gcd}\left(a_{j}, b_{j}\right)=1 .
$$

Here $h$ is the genus of the orbifold $\Sigma$, and $n=\operatorname{deg}(\mathcal{L})$ is the degree of $\mathcal{L}$. Just as for line bundles on a smooth Riemann surface, the Riemann-Roch theorem for orbifolds [77] states that the Euler character $\chi(\mathcal{L})$ is given in terms of the degree and the genus by

$$
\chi(\mathcal{L})=n+1-h,
$$


and similarly

$$
\chi\left(\mathcal{L}^{t}\right)=\operatorname{deg}\left(\mathcal{L}^{t}\right)+1-h .
$$

From (C.14), we see that the difference of Euler characters appearing in (C.11) is independent of $h$ and given by

$$
\delta(t)=\chi\left(\mathcal{L}^{t}\right)-\chi\left(\mathcal{L}^{-t}\right)=\operatorname{deg}\left(\mathcal{L}^{t}\right)-\operatorname{deg}\left(\mathcal{L}^{-t}\right) .
$$

If $\mathcal{L}$ is a line bundle of degree $d$ over a smooth Riemann surface, then $\operatorname{deg}\left(\mathcal{L}^{t}\right)=t d$, and $\delta(t)=2 t d$. However, on an orbifold the degree is not generally multiplicative,

$$
\operatorname{deg}\left(\mathcal{L}^{t}\right) \neq t \operatorname{deg}(\mathcal{L})
$$

and $\delta(t)$ is a more complicated arithmetic function which we must compute.

If $\mathcal{L}$ is characterized by isotropy invariants $b_{j}$ on $\Sigma$ as in (C.12), then $\mathcal{L}^{t}$ is characterized by isotropy invariants $b_{j}^{(t)}$ determined by the conditions

$$
b_{j}^{(t)} \equiv t b_{j} \bmod a_{j}, \quad 0 \leq b_{j}^{(t)}<a_{j} .
$$

In particular,

$$
\frac{b_{j}^{(t)}}{a_{j}}=\left\{\frac{t b_{j}}{a_{j}}\right\},
$$

where $\{\cdot\}$ denotes the fractional ${ }^{44}$ part of the given argument.

Now, unlike the degree, the first Chern class is multiplicative even for orbifold line bundles,

$$
c_{1}\left(\mathcal{L}^{t}\right)=t c_{1}(\mathcal{L})
$$

We also recall that the first Chern class of $\mathcal{L}^{t}$ is given by

$$
c_{1}\left(\mathcal{L}^{t}\right)=\operatorname{deg}\left(\mathcal{L}^{t}\right)+\sum_{j=1}^{N} \frac{b_{j}^{(t)}}{a_{j}} .
$$

\footnotetext{
${ }^{44}$ For $x \in \mathbb{R},\{x\}$ denotes the element in the interval $[0,1)$ such that $x-\{x\} \in \mathbb{Z}$.
} 
From (C.18) and (C.19) we therefore obtain

$$
\operatorname{deg}\left(\mathcal{L}^{t}\right)=t c_{1}(\mathcal{L})-\sum_{j=1}^{N}\left\{\frac{t b_{j}}{a_{j}}\right\},
$$

implying

$$
\delta(t)=2 t c_{1}(\mathcal{L})-\sum_{j=1}^{N}\left[\left\{\frac{t b_{j}}{a_{j}}\right\}-\left\{-\frac{t b_{j}}{a_{j}}\right\}\right] .
$$

Let us introduce another function $((\cdot))$ related to $\{\cdot\}$ by

$$
((x))=\left\{\begin{array}{ll}
\{x\}-\frac{1}{2} & \text { if } x \in \mathbb{R}-\mathbb{Z} \\
0 & \text { if } x \in \mathbb{Z}
\end{array} .\right.
$$

Clearly $((x+1))=((x))$ and $((-x))=-((x))$. In terms of $((\cdot))$, the sum in (C.22) can be rewritten as

$$
\delta(t)=2 t c_{1}(\mathcal{L})-2 \sum_{j=1}^{N}\left(\left(\frac{t b_{j}}{a_{j}}\right)\right) .
$$

Thus $\eta_{0}(s)$ is given explicitly by

$$
\eta_{0}(s)=2 \Delta_{G} \sum_{t=1}^{\infty}\left[\frac{c_{1}(\mathcal{L})}{t^{s-1}}-\sum_{j=1}^{N}\left(\left(\frac{t b_{j}}{a_{j}}\right)\right) \frac{1}{t^{s}}\right] .
$$

In passing to (C.25) from (C.11), we simply replace the sum over all roots $\beta$ (including those trivial roots associated to the Cartan subalgebra itself) by the prefactor $\Delta_{G}=\operatorname{dim} G$.

To evaluate $\eta_{0}(s)$ at $s=0$, we use the Hurwitz zeta-function $\zeta(s, a)$, defined by the sum

$$
\zeta(s, a)=\sum_{m=0}^{\infty} \frac{1}{(m+a)^{s}} .
$$

Here $s$ is a complex variable, and $a$ is a real parameter in the interval $0<a \leq 1$. As before, when the real part of $s$ is sufficiently large, the sum in (C.26) is absolutely convergent. Otherwise, $\zeta(s, a)$ is defined by analytic 
continuation in the $s$-plane. Clearly if $a=1$, then $\zeta(s, 1) \equiv \zeta(s)$ reduces to the standard Riemann zeta-function.

The Hurwitz zeta-function plays a role in evaluating (C.25) for the following elementary reason. If $f$ is any periodic function with integral period $L \in \mathbb{Z}$, then

$$
\begin{aligned}
\sum_{t=1}^{\infty} \frac{f(t)}{t^{s}} & =\sum_{l=1}^{L} \sum_{m=0}^{\infty} \frac{f(l+m L)}{(l+m L)^{s}} \\
& =\sum_{l=1}^{L} \frac{f(l)}{L^{s}} \zeta\left(s, \frac{l}{L}\right) .
\end{aligned}
$$

In passing to the second line in (C.27), we use the assumption $f(l+m L)=$ $f(l)$ and the definition of $\zeta(s, a)$ in (C.26).

Since the function $f_{j}(t)=\left(\left(\frac{t b_{j}}{a_{j}}\right)\right)$ is invariant under the shift $t \mapsto t+a_{j}$, we apply the identity in (C.27) to obtain

$$
\eta_{0}(s)=2 \Delta_{G}\left[c_{1}(\mathcal{L}) \zeta(s-1)-\sum_{j=1}^{N} \sum_{l=1}^{a_{j}-1}\left(\left(\frac{l b_{j}}{a_{j}}\right)\right) \frac{1}{a_{j}^{s}} \zeta\left(s, \frac{l}{a_{j}}\right)\right] .
$$

Naively, the sum in (C.28) runs over $l=1, \ldots, a_{j}$, but the summand vanishes identically when $l=a_{j}$, so we omit that term.

To evaluate $\eta_{0}(s)$ at $s=0$, we recall that $\zeta(-1)=-1 / 12$ and $\zeta(0, a)=$ $1 / 2-a$. See for instance Section 12 of [7] for a proof of the latter identity. Consequently, again using the function $((\cdot))$ in $($ C.23),

$$
\eta_{0}(0)=\frac{\Delta_{G}}{6}\left[-c_{1}(\mathcal{L})+12 \sum_{j=1}^{N} \sum_{l=1}^{a_{j}-1}\left(\left(\frac{l}{a_{j}}\right)\right)\left(\left(\frac{l b_{j}}{a_{j}}\right)\right)\right] .
$$

Finally, as explained for instance in Section 3 of [6], the Dedekind sum $s(b, a)$ can be described not only trigonometrically as in (7.59) but also arithmetically in terms of $((\cdot))$ as

$$
s(b, a)=\sum_{l=1}^{a-1}\left(\left(\frac{l}{a}\right)\right)\left(\left(\frac{l b}{a}\right)\right) .
$$


Hence

$$
\eta_{0}(0)=\frac{\Delta_{G}}{6}\left[-c_{1}(\mathcal{L})+12 \sum_{j=1}^{N} s\left(b_{j}, a_{j}\right)\right]
$$

For the Seifert homology spheres considered in Section $7.2, c_{1}(\mathcal{L})=d / \mathrm{P}$, so we obtain the formula for $\eta_{0}(0)$ in (7.109).

\section{Appendix D A note on the extended denominator formula}

In this appendix, we establish the slight extension of the Weyl denominator formula needed to identify the two expressions for $\mathrm{B}_{\alpha}(\phi)$ in $(7.157)$ and (7.159).

Briefly, the extended denominator formula states

$$
\begin{aligned}
& \sum_{w \in \mathfrak{W}_{\alpha}}(-1)^{w} \mathrm{e}^{\langle w \cdot(\alpha+\rho), \phi\rangle} \\
& \quad=\mathrm{e}^{\left\langle\alpha+\rho^{[\alpha]}, \phi\right\rangle} \cdot \prod_{\beta_{\perp}>0}\left[2 \sinh \left(\frac{\left\langle\beta_{\perp}, \phi\right\rangle}{2}\right)\right], \quad \phi \in \mathfrak{t} .
\end{aligned}
$$

Here $\alpha>0$ is a positive weight of $G, \rho$ is the standard Weyl vector given by half the sum of the positive roots of $G$, and the alternating sum on the left side of (D.1) runs over elements of the Weyl group $\mathfrak{W}_{\alpha}$ of the stabilizer $G_{\alpha} \subseteq G$ of $\alpha$ under the adjoint action of $G$. If $\alpha$ is regular so that $G_{\alpha}=T$ is a maximal torus, then $\mathfrak{W}_{\alpha}$ is trivial by convention.

On the right side of (D.1), $\rho^{[\alpha]}$ is defined as in (7.130) by

$$
\rho^{[\alpha]}=\frac{1}{2} \sum_{\left(\beta_{+}, \alpha\right)>0} \beta_{+}
$$

where the sum runs over all roots $\beta_{+}$of $G$ such that $\left(\beta_{+}, \alpha\right)>0$. If $\alpha$ is regular, $\rho^{[\alpha]}$ is therefore the standard Weyl vector $\rho$. At the other extreme, if $\alpha=0$, then $\rho^{[\alpha]}=0$ as well. Finally, the product on the right side of (D.1) runs over all positive roots $\beta_{\perp}>0$ of $G$ such that $\left(\beta_{\perp}, \alpha\right)=0$. For future reference, we note that the roots $\beta_{\perp}$ are precisely the roots of $G_{\alpha}$. When $\alpha$ is regular, the product over $\beta_{\perp}>0$ is taken to be 1 . With this convention, the identity in (D.1) holds trivially for regular weights. 
At the opposite extreme, for $\alpha=\rho^{[\alpha]}=0$, the identity in (D.1) reduces to the standard product formula for the Weyl denominator $\mathrm{A}_{\rho}$,

$$
\mathrm{A}_{\rho}(\phi)=\sum_{w \in \mathfrak{W}}(-1)^{w} \mathrm{e}^{\langle w \cdot \rho, \phi\rangle}=\prod_{\beta>0}\left[2 \sinh \left(\frac{\langle\beta, \phi\rangle}{2}\right)\right], \quad \phi \in \mathfrak{t} .
$$

where the sum in (D.3) runs over the full Weyl group $\mathfrak{W}$ of $G$ and the product runs over all positive $\operatorname{roots} \beta>0$.

Our proof of the extended denominator formula (D.1) is an immediate generalization of the proof of (D.3) offered in Lemma 24.3 of [52]. Nonetheless, for sake of completeness we reproduce that argument here.

Let us first introduce some temporary notation for the left and right sides of (D.1),

$$
\begin{aligned}
\mathrm{B}_{\alpha} & =\sum_{w \in \mathfrak{W}_{\alpha}}(-1)^{w} \mathrm{e}^{w \cdot(\alpha+\rho)}, \\
\mathrm{B} & =\mathrm{e}^{\left(\alpha+\rho^{[\alpha]}\right)} \cdot \prod_{\beta_{\perp}>0}\left[2 \sinh \left(\frac{\beta_{\perp}}{2}\right)\right],
\end{aligned}
$$

where for sake of brevity we omit the variable $\phi \in \mathfrak{t}$ henceforth.

Clearly $\mathrm{B}_{\alpha}$ is alternating under the action of $\mathfrak{W}_{\alpha}$. We also observe that $\mathrm{B}$ is alternating under $\mathfrak{W}_{\alpha}$. By definition, $\alpha$ and $\rho^{[\alpha]}$ are invariant under $\mathfrak{W}_{\alpha}$, and the product $\prod_{\beta_{\perp}>0}\left[2 \sinh \left(\beta_{\perp} / 2\right)\right]$ otherwise appearing in $\mathrm{B}$ is alternating. Here we recall that $\mathfrak{W}_{\alpha}$ is generated by reflections in the simple roots of $G_{\alpha}$. Hence the quotient $\mathrm{B}_{\alpha} / \mathrm{B}$ is invariant under $\mathfrak{W}_{\alpha}$.

Next, we observe trivially that

$$
\begin{aligned}
\mathrm{B} & =\mathrm{e}^{\left(\alpha+\rho^{[\alpha]}\right)} \cdot\left[\exp \left(\frac{1}{2} \sum_{\beta_{\perp}>0} \beta_{\perp}\right) \cdot \prod_{\beta_{\perp}>0}\left(1-\mathrm{e}^{-\beta_{\perp}}\right)\right] \\
& =\mathrm{e}^{(\alpha+\rho)} \cdot \prod_{\beta_{\perp}>0}\left(1-\mathrm{e}^{-\beta_{\perp}}\right)
\end{aligned}
$$

where we use the identity

$$
\rho=\rho^{[\alpha]}+\frac{1}{2} \sum_{\beta_{\perp}>0} \beta_{\perp}
$$


Inverting the latter expression for B in (D.5), we find

$$
\mathrm{B}^{-1}=\mathrm{e}^{-(\alpha+\rho)} \cdot \prod_{\beta_{\perp}>0}\left[\sum_{m=0}^{\infty} \mathrm{e}^{-m \beta_{\perp}}\right],
$$

where we use the standard series presentation for $1 /\left(1-\mathrm{e}^{-\beta_{\perp}}\right)$. So as a formal series, the quotient $\mathrm{B}_{\alpha} / \mathrm{B}$ becomes

$$
\frac{\mathrm{B}_{\alpha}}{\mathrm{B}}=\sum_{w \in \mathfrak{W}_{\alpha}}(-1)^{w} \mathrm{e}^{(w \cdot \rho-\rho)} \cdot \prod_{\beta_{\perp}>0}\left[\sum_{m=0}^{\infty} \mathrm{e}^{-m \beta_{\perp}}\right] .
$$

In arriving at (D.8), we use that $w \cdot(\alpha+\rho)=\alpha+w \cdot \rho$, as $\alpha$ is invariant under $\mathfrak{W}_{\alpha}$.

We now observe that $(w \cdot \rho-\rho)<0$ is strictly negative for all non-trivial elements $w \in \mathfrak{W}_{\alpha}$. Thus the expression on the right in (D.8) is a sum of exponentials, all of whose arguments, if non-trivial, are strictly negative weights of $G_{\alpha} \cdot{ }^{45}$ On the other hand, the sum of exponentials in (D.8) is by construction invariant under $\mathfrak{W}_{\alpha}$. Since $\mathfrak{W}_{\alpha}$ acts simply transitively on the Weyl chambers of $G_{\alpha}$, these two observations are consistent only if $\mathrm{B}_{\alpha} / \mathrm{B}$ is constant as a function on $\mathfrak{t}$.

Finally, comparing the leading terms of the sum and product in (D.4), we easily verify that $\mathrm{B}_{\alpha}=\mathrm{B}$.

\section{References}

[1] D.H. Adams, The semi-classical approximation for the ChernSimons partition function, Phys. Lett. B 417 (1998), 53-60, arXiv:hep-th/9709147.

[2] M. Aganagic, A. Neitzke and C. Vafa, BPS microstates and the open topological string wave function, arXiv:hep-th/0504054.

[3] M. Aganagic, H. Ooguri, N. Saulina and C. Vafa, Black holes, q-deformed 2d Yang-Mills, and non-perturbative topological strings, arXiv:hep-th/0411280.

[4] A. Alekseev, L.D. Faddeev and S.L. Shatashvili, Quantization of symplectic orbits of compact lie groups by means of the functional integral, J. Geom. Phys. 5 (1989), 391-406.

\footnotetext{
${ }^{45}$ Since $\alpha$ is fixed by $\mathfrak{W}_{\alpha}$, the inner-product $(\alpha, w \cdot \rho-\rho)$ vanishes for $w \in \mathfrak{W}_{\alpha}$, so the difference $(w \cdot \rho-\rho)$ is indeed a weight of the subgroup $G_{\alpha} \subseteq G$.
} 
"ATMP-17-1-A1-BEA" — 2013/5/15 — 19:32 — page 234 — \#234

[5] O. Alvarez, I.M. Singer and P. Windey, Quantum mechanics and the geometry of the Weyl character formula, Nucl. Phys. B 337 (1990), 467-486.

[6] T. Apostol, Modular functions and Dirichlet series in number theory, Springer-Verlag, New York, 1976.

[7] T. Apostol, Introduction to analytic number theory, Springer-Verlag, New York, 1976.

[8] X. Arsiwalla, R. Boels, M. Mariño and A. Sinkovics, Phase transitions in q-deformed 2d Yang-Mills theory and topological strings, Phys. Rev. D 73 (2006), 026005; arXiv:hep-th/0509002.

[9] M.F. Atiyah, Circular symmetry and stationary-phase approximation, in Colloquium in honor of Laurent Schwartz, 1, Astérisque, 131 (1985), 43-59.

[10] M. Atiyah, The geometry and physics of knots, Cambridge University Press, Cambridge, 1990.

[11] M. Atiyah and R. Bott, The moment map and equivariant cohomology, Topology 23 (1984), 1-28.

[12] M. Atiyah and R. Bott, Yang-Mills equations over Riemann surfaces, Phil. Trans. R. Soc. Lond. A308 (1982), 523-615.

[13] M. Atiyah, On framings of three-manifolds, Topology 29 (1990), 1-7.

[14] M.F. Atiyah, V. Patodi and I. Singer, Spectral asymmetry and Riemannian geometry, I, II, III, Math. Proc. Camb. Phil. Soc. 77 (1975), 43-69; 78 (1975), 405-432; 79 (1976), 71-99.

[15] M.F. Atiyah and R. Bott, A Lefschetz fixed point formula for elliptic complexes, II, Ann. Math. 88 (1968), 451-491.

[16] A.P. Balachandran, S. Borchardt and A. Stern, Lagrangian and hamiltonian descriptions of Yang-Mills particles, Phys. Rev. D 17 (1978), 3247-3256.

[17] L. Baulieu, A. Losev and N. Nekrasov, Chern-Simons and twisted supersymmetry in various dimensions, Nucl. Phys. B 522 (1998), 82-104; arXiv:hep-th/9707174.

[18] C. Beasley and E. Witten, Non-abelian localization for Chern-Simons theory, J. Differ. Geom. 70 (2005), 183-323; arXiv:hep-th/0503126.

[19] D.E. Blair, Riemannian geometry of contact and symplectic manifolds, Birkhäuser, Boston, 2002.

[20] M. Blau and G. Thompson, Chern-Simons Theory on $S^{1}$-bundles: Abelianisation and q-deformed Yang-Mills theory, JHEP 0605 (2006), 003; arXiv:hep-th/0601068.

[21] M. Blau and G. Thompson, Lectures on 2-d gauge theories: topological aspects and path integral techniques, in 'Proceedings of the 1993 
"ATMP-17-1-A1-BEA" — 2013/5/15 - 19:32 — page 235 — \#235

LOCALIZATION FOR WILSON LOOPS IN CHERN-SIMONS

Trieste Summer School in High Energy Physics and Cosmology', ed. by E. Gava et al., ICTP Series in Theoretical Physics, vol. 10, World Scientific, Hackensack, NJ, 1994; arXiv:hep-th/9310144.

[22] M. Blau and G. Thompson, Localization and diagonalization: a review of functional integral techniques for low dimensional gauge theories and topological field theories, J. Math. Phys. 36 (1995), 2192-2236; arXiv:hep-th/9501075.

[23] H. Boden, C. Herald, P. Kirk and E. Klassen, Gauge theoretic invariants of Dehn surgeries on knots, Geom. Topol. 5 (2001), 143-226; arXiv:math.GT/9908020.

[24] R. Bott, Homogeneous vector bundles, Ann. Math. 66 (1957), 203-248.

[25] R. Bott and L. Tu, Differential forms in algebraic topology, SpringerVerlag, New York, 1982.

[26] T. Buscher, Path integral derivation of quantum duality in nonlinear sigma models, Phys. Lett. B 201 (1988), 466-472.

[27] N. Caporaso, M. Cirafici, L. Griguolo, S. Pasquetti, D. Seminara and R.J. Szabo, Topological strings and large $N$ phase transitions, II: chiral expansion of q-deformed Yang-Mills theory, JHEP 01 (2006), 036; arXiv:hep-th/0511043.

[28] N. Caporaso, M. Cirafici, L. Griguolo, S. Pasquetti, D. Seminara and R.J. Szabo, Topological strings and large $N$ phase transitions, I: nonchiral expansion of q-deformed Yang-Mills theory, JHEP 01 (2006), 035; arXiv:hep-th/0509041.

[29] S. Cordes, G.W. Moore and S. Ramgoolam, Lectures on 2-d YangMills theory, equivariant cohomology and topological field theories, Nucl. Phys. Proc. Suppl. 41 (1995), 184-244; arXiv: hep-th/9411210.

[30] G. Daskalopoulos and R. Wentworth, Geometric quantization for the moduli space of vector bundles with parabolic structure, in Geometry, Topology, and Physics (Campinas, 1996), ed. B. Apanasov et al., pp. 119-155, de Gruyter, Berlin, 1997.

[31] S. de Haro, A note on knot invariants and q-deformed 2d Yang-Mills, Phys. Lett. B 634 (2006), 78-83; arXiv: hep-th/0509167.

[32] S. de Haro and M. Tierz, Discrete and oscillatory matrix models in Chern-Simons theory, Nucl. Phys. B 731 (2005), 225-241; arXiv: hep-th/0501123.

[33] S. de Haro, Chern-Simons theory, 2d Yang-Mills, and Lie algebra wanderers, Nucl. Phys. B 730 (2005), 312-351; arXiv:hep-th/0412110.

[34] S. de Haro, Chern-Simons theory in lens spaces from 2d Yang-Mills on the cylinder, JHEP 08 (2004), 041; arXiv:hep-th/0407139. 
"ATMP-17-1-A1-BEA" — 2013/5/15 — 19:32 — page 236 — \#236

[35] S. de Haro and M. Tierz, Brownian motion, Chern-Simons theory, and 2d Yang-Mills, Phys. Lett. B 601 (2004), 201-208; arXiv:hep-th/0406093.

[36] S. de Haro, S. Ramgoolam and A. Torrielli, Large- $N$ expansion of q-deformed two-dimensional Yang-Mills theory and Hecke algebras, Commun. Math. Phys. 273 (2007), 317-355; arXiv: hep-th/0603056.

[37] E. Witten, Dynamics of quantum field theory, in Quantum Fields and Strings: A Course for Mathematicians, Vol. 2, ed. by P. Deligne et al., American Mathematical Society, Providence, Rhode Island, 1999.

[38] E. Witten, Index of Dirac operators, in 'Quantum Fields and Strings: A Course for Mathematicians', vol. 1, eds. P. Deligne et al., American Mathematical Society, Providence, Rhode Island, 1999.

[39] D. Diakonov and V.Y. Petrov, A formula for the Wilson loop, Phys. Lett. B 224 (1989), 131-135.

[40] P. Di Francesco, P. Mathieu and D. Sénéchal, Conformal field theory, Springer-Verlag, New York, 1997.

[41] R. Dijkgraaf and H. Fuji, The volume conjecture and topological strings, arXiv:0903.2084 [hep-th].

[42] S.K. Donaldson, Moment maps and diffeomorphisms, in 'Sir Michael Atiyah: A Great Mathematician of the Twentieth Century', Asian J. Math. 3 (1999), 1-15.

[43] S.K. Donaldson, Gluing techniques in the cohomology of moduli spaces, in 'Topological Methods in Modern Mathematics', eds. L. Goldberg and A. Phillips, Publish or Perish, Houston, Texas, 1993, 137-170.

[44] J.-M. Drezet and M.S. Narasimhan, Groupe de Picard des variétés de modules de fibrés semi-stables sur les courbes algébriques, Invent. Math. 97 (1989), 53-94.

[45] J. Dubois and R. Kashaev, On the asymptotic expansion of the colored jones polynomial for torus knots, Math. Ann. 339 (2007), 757-782; arXiv:math.GT/0510607.

[46] J.J. Duistermaat and G.J. Heckman, On the variation in the cohomology of the symplectic form of the reduced phase space, Invent. Math. 69 (1982), 259-268; Addendum, Invent. Math. 72 (1983), 153-158.

[47] S. Elitzur, G. Moore, A. Schwimmer and N. Seiberg, Remarks on the canonical quantization of the Chern-Simons-Witten theory, Nucl. Phys. B 326 (1989), 108-134.

[48] J.B. Etnyre, Introductory lectures on contact geometry, in Topology and Geometry of Manifolds (Athens, GA 2001), Proc. Symp. 
"ATMP-17-1-A1-BEA" — 2013/5/15 — 19:32 — page 237 — \#237

LOCALIZATION FOR WILSON LOOPS IN CHERN-SIMONS

on Pure Mathematics 71, Amer. Math. Soc., Providence, RI, 2003; arXiv:math.SG/0111118.

[49] J.B. Etnyre, Legendrian and transversal knots, in 'Handbook of Knot Theory', eds. W. Menasco and M. Thistlethwaite, Elsevier, Amsterdam, 2005, 105-185; arXiv:math.SG/0306256.

[50] D.S. Freed, Remarks on Chern-Simons theory, Bull. Amer. Math. Soc. 46 (2009), 221-254; arXiv:0808.2507 [math.AT].

[51] D. Freed and R. Gompf, Computer calculation of Witten's 3-Manifold invariant, Commun. Math. Phys. 141 (1991), 79-117.

[52] W. Fulton and J. Harris, Representation theory: a first course, Springer, New York, 1991.

[53] M. Furuta and B. Steer, Seifert fibred homology 3-spheres and the Yang-Mills equations on Riemann surfaces with marked points, Adv. Math. 96 (1992), 38-102.

[54] H. Geiges, Contact geometry, in 'Handbook of Differential Geometry', Vol. II, eds. F. Dillen and L. Verstraelen, Elsevier, Amsterdam, 2006, 315-382; arXiv:math.SG/0307242.

[55] V. Guillemin and S. Sternberg, Symplectic techniques in physics, Cambridge University Press, Cambridge, 1984.

[56] V. Guillemin and S. Sternberg, Supersymmetry and equivariant de Rham theory, Springer, Berlin, 1999.

[57] A. Hahn, Chern-Simons models on $S^{2} \times S^{1}$, torus gauge fixing, and link invariants I,II, J. Geom. Phys. 53 (2005), 275-314

—,J. Geom. Phys. 58 (2008) 1124-1136.

[58] M. Hamilton and L. Jeffrey, Symplectic fibrations and RiemannRoch numbers of reduced spaces, Q. J. Math 56 (2005), 541-552; arXiv:math.SG/0403004.

[59] F. Hirzebruch, Topological methods in algebraic geometry, 3rd ed., Springer-Verlag, New York, 1966.

[60] N. Itzhaki, Anyons, 't Hooft loops, and a generalized connection in three dimensions, Phys. Rev. D 67 (2003), 065008; arXiv:hep-th/0211140.

[61] D. Jafferis and J. Marsano, A DK phase transition in q-deformed Yang-Mills on $S^{2}$ and topological strings, arXiv:hep-th/0509004.

[62] L. Jeffrey, Chern-Simons-Witten invariants of lens spaces and torus bundles, and the semiclassical approximation, Commun. Math. Phys. 147 (1992), 563-604.

[63] L. Jeffrey, Extended moduli spaces of flat connections on Riemann surfaces, Math. Ann. 298 (1994), 667-692. 
"ATMP-17-1-A1-BEA" — 2013/5/15 — 19:32 — page 238 — \#238

[64] L. Jeffrey and F. Kirwan, Intersection theory on moduli spaces of holomorphic bundles of arbitrary rank on a Riemann surface, Ann. Math. 148 (1998), 109-196; arXiv:alg-geom/9608029.

[65] L. Jeffrey, The Verlinde formula for parabolic bundles, J. Lond. Math. Soc. 63 (2001), 754-768; arXiv:math.AG/0003150.

[66] L. Jeffrey, Y-H. Kiem, F. Kirwan, and J. Woolf, Intersection pairings on singular moduli spaces of bundles over a Riemann surface and their partial desingularisations, Transform. Groups 11 (2006), 439-494; arXiv:math.AG/0505362.

[67] L. Jeffrey and B. McLellan, Non-abelian localization for U(1) ChernSimons theory; arXiv:0903.5093 [math.DG].

[68] V.F.R. Jones, Hecke algebra representations of braid groups and link polynomials, Ann. Math. 126 (1987), 335-388.

[69] S. Garoufalidis, Relations among 3-manifold invariants, Ph.D. thesis, University of Chicago, 1992.

[70] S. Gukov and E. Witten, Gauge theory, ramification, and the geometric Langlands program, in 'Current Developments in Mathematics', 2006, eds. D. Jerison et al., pp. 35-180, Int. Press, Somerville, Massachusetts, 2008; arXiv:hep-th/0612073.

[71] A. Hatcher, Algebraic topology, Cambridge University Press, Cambridge, 2002.

[72] S. Helgason, Differential geometry, Lie groups, and symmetric spaces, Academic Press, San Diego, 1978.

[73] J. Isidro, J. Labastida and A. Ramallo, Polynomials for torus links from Chern-Simons gauge theories, Nucl. Phys. B 398 (1993), 187-236; arXiv: hep-th/9210124.

[74] R. Lawrence and L. Rozansky, Witten-Reshetikhin-Turaev invariants of Seifert manifolds, Commun. Math. Phys. 205 (1999), 287-314.

[75] A. Kapustin, B. Willett and I. Yaakov, Exact results for Wilson loops in superconformal Chern-Simons theories with matter; arXiv:0909.4559 [hep-th].

[76] R.M. Kashaev and O. Tirkkonen, A proof of the volume conjecture for torus knots, J. Math. Sci (NY) 115 (2003), 2033-2036; arXiv:math.GT/9912210.

[77] T. Kawasaki, The Riemann-Roch Theorem for Complex $V$-manifolds, Osaka J. Math. 16 (1979), 151-159.

[78] A.A. Kirillov, Elements of the theory of representations, Transl. by E. Hewitt, Springer-Verlag, Berlin, 1976.

[79] E. Klassen, Representations of knot groups in $S U(2)$, Trans. Amer. Math. Soc. 326 (1991), 795-828. 
"ATMP-17-1-A1-BEA" — 2013/5/15 — 19:32 — page 239 — \#239

[80] J. Labastida, P. Llatas and A. Ramallo, Knot operators in ChernSimons gauge theory, Nucl. Phys. B 348 (1991), 651-692.

[81] M. Mariño, Chern-Simons theory, matrix integrals, and perturbative three-manifold invariants, Commun. Math. Phys. 253 (2004), 25-49; arXiv: hep-th/0207096.

[82] J. Martinet, Formes de contact sur les varietétés de dimension 3, Springer Lecture Notes in Mathematics 209, (1971), 142-163.

[83] V.B. Mehta and C.S. Seshadri, Moduli of vector bundles on curves with parabolic structures, Math. Ann. 248 (1980), 205-239.

[84] A.A. Migdal, Recursion equations in gauge field theories, Zh. Eksp. Teor. Fiz. 69 (1975), 810-822 [Sov. Phys. JETP 42 (1975), 413-418].

[85] L. Moser, Elementary surgery along a torus knot, Pacific J. Math 38 (1971), 737-745.

[86] H. Murakami, An introduction to the volume conjecture and Its generalizations, Acta Math. Vietnam, 33 (2008), 219-253; arXiv:0802.0039 [math.GT].

[87] M. Narasimhan and C. Seshadri, Stable and unitary vector bundles on a compact Riemann surface, Ann. of Math. 82 (1965), 540-567.

[88] N. Nekrasov, Four-Dimensional holomorphic theories, PhD thesis, Princeton University, 1996.

[89] L.I. Nicolaescu, Finite Energy Seiberg-Witten moduli spaces on fourmanifolds bounding Seifert fibrations, Comm. Anal. Geom. 8 (2000), 1027-1096; arXiv:dg-ga/9711006.

[90] P. Orlik, Seifert Manifolds, Lecture Notes in Mathematics 291, eds. A. Dold and B. Eckmann, Springer-Verlag, Berlin, 1972.

[91] Problems on invariants of knots and 3-Manifolds, ed. T. Ohtsuki with an introduction by J. Roberts, in Invariants of Knots and 3-manifolds (Kyoto, 2001), 377-572, Geom. Topol. Monogr. 4, Geom. Topol. Publ., Coventry, 2002; arXiv:math.GT/0406190.

[92] P.-E. Paradan, The moment map and equivariant cohomology with generalized coefficients, Topology 39 (2000), 401-444.

[93] V. Pestun, Localization of gauge theory on a four-sphere and supersymmetric Wilson loops; arXiv:0712.2824 [hep-th].

[94] A. Pressley and G. Segal, Loop groups, Clarendon Press, Oxford, 1986.

[95] N. Reshetikhin and V. G. Turaev, Invariants of 3-manifolds via link polynomials and quantum groups, Invent. Math. 103 (1991), 547-597.

[96] M. Rocek and E. Verlinde, Duality, quotients, and currents, Nucl. Phys. B 373 (1992), 630-646; arXiv:hep-th/9110053.

[97] I. Satake, On a generalization of the notion of manifold, Proc. Natl. Acad. Sci. USA 42 (1956), 359-363. 
"ATMP-17-1-A1-BEA" — 2013/5/15 — 19:32 — page $240 — \# 240$

[98] I. Satake, The Gauss-Bonnet Theorem for V-manifolds, J. Math. Soc. Japan 9 (1957), 464-492.

[99] M. Stone, Supersymmetry and the quantum mechanics of spin, Nucl. Phys. B 314 (1989), 557-586.

[100] C. Teleman, K-theory of the moduli space of bundles on a surface and deformations of the Verlinde algebra, in Topology, Geometry, and Quantum Field Theory: Proceedings of the Symp. in Honor of the 60th Birthday of Graeme Segal, ed. by U. Tillmann, LMS Lecture Notes 308, Cambridge University Press, Cambridge, 2004.

[101] C. Teleman and C.T. Woodward, The index formula for the moduli of G-bundles; arXiv:math.AG/0312154.

[102] C. Teleman, The quantization conjecture revisited, Ann. of Math. 152 (2000), 1-43; arXiv:math.AG/9808029.

[103] J. Weitsman, Fermionization and convergent perturbation expansions in Chern-Simons gauge theory; arXiv:0902.0097 [math-ph].

[104] E. Witten, On S-duality in abelian gauge theory, Selecta Math. 1 (1995), 383-410; arXiv:hep-th/9505186.

[105] E. Witten, Quantum field theory and the Jones polynomial, Commun. Math. Phys. 121 (1989), 351-399.

[106] E. Witten, On quantum gauge theories in two-dimensions, Commun. Math. Phys. 141 (1991), 153-209.

[107] E. Witten, Gauge theories and integrable lattice models, Nucl. Phys. B 322 (1989), 629-697.

[108] E. Witten, Two-dimensional gauge theories revisited, J. Geom. Phys. 9 (1992), 303-368; arXiv:hep-th/9204083.

[109] N.M.J. Woodhouse, Geometric quantization, 2nd Edn., Clarendon Press, Oxford, 1992.

[110] C.T. Woodward, Localization for the norm-square of the moment map and the two-dimensional Yang-Mills integral, J. Symplectic Geom. 3 (2005), 17-54; arXiv:math.SG/0404413. 